\title{
Apple Pomace as a Novel Aid for Western Diet-Induced Nonalcoholic Fatty Liver Disease in Young Female Sprague Dawley Rats
}

\author{
R. Chris Skinner \\ West Virginia University, rcskinner@mix.wvu.edu
}

Follow this and additional works at: https://researchrepository.wvu.edu/etd

Part of the Molecular, Genetic, and Biochemical Nutrition Commons

\footnotetext{
Recommended Citation

Skinner, R. Chris, "Apple Pomace as a Novel Aid for Western Diet-Induced Nonalcoholic Fatty Liver Disease in Young Female Sprague Dawley Rats" (2019). Graduate Theses, Dissertations, and Problem Reports. 3916.

https://researchrepository.wvu.edu/etd/3916

This Dissertation is protected by copyright and/or related rights. It has been brought to you by the The Research Repository @ WVU with permission from the rights-holder(s). You are free to use this Dissertation in any way that is permitted by the copyright and related rights legislation that applies to your use. For other uses you must obtain permission from the rights-holder(s) directly, unless additional rights are indicated by a Creative Commons license in the record and/ or on the work itself. This Dissertation has been accepted for inclusion in WVU Graduate Theses, Dissertations, and Problem Reports collection by an authorized administrator of The Research Repository @ WVU. For more information, please contact researchrepository@mail.wvu.edu.
} 
Apple Pomace as a Novel Aid for Western Diet-Induced Nonalcoholic Fatty Liver Disease in Young Female Sprague Dawley Rats

\author{
R. Chris Skinner \\ Dissertation submitted to the \\ Davis College of Agriculture, Forestry, and Consumer Sciences \\ at West Virginia University \\ Doctor of Philosophy in \\ Animal and Food Science \\ emphasis in Human Nutrition \\ Janet Tou, PhD, Chair \\ Vagner Benedito, $\mathrm{PhD}$ \\ Joseph Gigliotti, PhD \\ Kang Mo Ku, PhD \\ Joseph Moritz, PhD
}

Division of Animal and Nutritional Sciences

Morgantown, WV

2019

Keywords: apple pomace, NAFLD, functional food, Western diet, steatosis, inflammation Copyright 2019 R. Chris Skinner 


\begin{abstract}
Apple Pomace as a Novel Nutritional Aid for Western Diet-Induced Nonalcoholic Fatty Liver Disease in Young Female Sprague Dawley Rats
\end{abstract}

\title{
R. Chris Skinner
}

Apple pomace is a "waste" byproduct of apple processing that causes environmental pollution and is costly to dispose of. Yet, apple pomace is rich in dietary fibers and antioxidants. Analysis of apple pomace's nutritional profile indicates suitability as a potential dietary treatment for nonalcoholic fatty liver disease (NAFLD) and the more severe non-alcoholic steatohepatitis (NASH). NAFLD is the most prevalent liver disease in the world with prevalence and severity expected to increase in both adults and children. Currently, there is no approved drug treatment for NAFLD and therefore, dietary intervention is the primary treatment. The study objectives were to determine the effect of apple pomace consumption on diet-induced NAFLD, NASH and renal and bone health using a rodent model. Growing (aged 22-29 d) female Sprague-Dawley rats ( $n=8 /$ group) were fed ad libitum diets consisting of AIN-93G, AIN-93G with $10 \%$ apple pomace substitution (AIN/AP), Western diet (45\% fat, 34\% sucrose), or Western diet with 10\% apple pomace substitution (Western/AP) for 8 weeks. Results showed Western diet consumption increased $(p<0.0001)$ gonadal adipose weight independent of body weight differences. Rats consuming Western diet showed histological evidence of hepatic fat infiltration and inflammation characterizing NAFLD and progression to NASH. Fatty acid analysis by gas chromatography showed increase $(p \leq 0.05)$ hepatic palmitic, palmitoleic, and oleic acid content in rats consuming Western diet was attenuated by apple pomace. Gonadal adipose tissue fatty acid analysis showed rats consuming a Western diet to have significantly reduced palmitic, stearic, and oleic acid compared to all diet groups. These results suggest saturated and monounsaturated fatty acids from the adipose tissue are being transported to the liver, resulting in increased hepatic fat deposition. Additionally, hepatic gene expression by real time-quantitative polymerase chain 
reaction (RT-qPCR) showed rats consuming Western diet upregulated hepatic expression of diacylglycerol O-acyltransferase 2 (DGAT2), which was attenuated by apple pomace. Rats consuming Western diets also had upregulated nuclear factor kappa-light chain enhancer of activated B cells (NFKB) and interleukin-6 (IL-6). Further, gonadal adipose tissue expression of $\mathrm{NFKB}, \mathrm{IL}-6$, and tumor necrosis factor alpha (TNFa) was significantly upregulated compared to all groups contributing to progression of NAFLD to NASH. The results suggest increased gonadal adipose also increased transport of inflammatory cytokines, resulting in NASH progression. Apple pomace attenuated Western diet-induced NAFLD due to the high fiber content in apple pomace increasing $(p \leq 0.02)$ serum bile acids. Caloric substitution with apple pomace also attenuated Western diet-induced progression to NASH. High polyphenol content in apple pomace resulted in significantly increased serum total antioxidants and decreased urinary antioxidants. The calcium and fructose content in apple pomace showed no significant effects in indices of renal or bone health. Collectively, the study results showed caloric substitution of a healthy or Western diet with $10 \%$ apple pomace attenuated NAFLD, progression to NASH, and was safe for renal and bone health. Therefore, apple pomace has potential to be repurposed for human consumption as a sustainable functional food and as a nutritional aid to promote liver health. 


\section{Acknowledgments}

I would like to thank my parents for continually supporting and encouraging me over the course of my schooling. Without your guidance and patience, I would surely not be the person, student, or scholar I am today. Thank you for all that you have done to prepare and support me over the course of my doctoral studies, before, and all you will do after. I love you both. I would also like to thank my girlfriend, Rebekah Honce, soon to be PhD. You have been the best girlfriend, support system, stress reliever, editor, therapist, and friend since we began dating. You have helped me grow as a person and as a scientist and I will forever be grateful you are a part of my life. I love my Bek.

Thank you to Janet Tou for accepting me into your lab, keeping me focused, allowing me to pursue my interests, and helping me to shape my future. I am very lucky to have enrolled in your course during the course of my Master's, and even more to have been your student the past three years. Thank you to my committee for the aid in methods and analysis, being available for questions and conversations, and for conversations had regarding my project and my future. Thank you, Vagner Benedito, Joey Gigliotti, Joe Moritz, and Kang Mo Ku. Thank you to friends and family members who have continually supported me, providing crucial mental breaks from science and excellent extracurricular adventures. Thank you to my lab mates, particularly Derek Warren for all your help, and all the interns who have assisted in my study. Thank you to mentors past, including Greg Popovich, Paul Chantler, and Amy Kuhn, who helped to shape my interests in the health sciences and were essential to my pursuing a PhD and a career in academics. Thank you to Swilled Dog Hard Cider Company for providing the apple pomace used in my project. Thank you to Office of the Provost and the WVU Foundation for funding my doctoral studies. Thank you to all others who have aided me in educational journey. Thank you to God. Thank you to West Virginia and West Virginia University. 


\section{TABLE OF CONTENTS}

$\begin{array}{ll}\text { Abstract } & \text { ii }\end{array}$

Acknowledgments iv

Introduction 1

$\begin{array}{lll}1.0 & \text { Literature Review } & 7\end{array}$

1.0.1 A Comprehensive Analysis of the Composition, Health Benefits, and Safety of Apple Pomace

$\begin{array}{ll}\text { 1.0.1 Abstract } & 8\end{array}$

$\begin{array}{ll}1.0 .2 \text { Introduction } & 9\end{array}$

1.0.3 Nutrient Composition of Apple Pomace $\quad 9$

1.0.4 Macronutrients 10

1.0.5 Micronutrients $\quad 13$

1.0.6 Health Benefits of Apple Pomace Consumption 18

1.0.7 Safety of Apple Pomace Consumption 26

1.0.8 Potential Food Uses 32

1.0.9 Conclusion 33

1.0.10 References

1.1 Diet-induced non-alcoholic fatty liver disease: a brief review 58

1.1.1 Etiology 58

$\begin{array}{ll}1.1 .2 \text { Diagnosis } & 61\end{array}$

1.1.3 Treatment $\quad 62$

1.1.4 Conclusions $\quad 64$

1.1.5 References $\quad 65$

$\begin{array}{ll}2.0 \text { Study Objectives and Hypotheses } & 77\end{array}$

$\begin{array}{lll}3.0 & \text { Chapter } 1 & 78\end{array}$

Apple pomace consumption favorably alters hepatic lipid metabolism in young female $\begin{array}{ll}\text { Sprague-Dawley rats fed a Western diet } & 78\end{array}$

$\begin{array}{ll}3.1 \text { Abstract } & 79\end{array}$

3.2. Introduction $\quad 80$

3.3. Materials and Methods $\quad 81$

$\begin{array}{ll}3.4 \text { Results } & 87\end{array}$

3.5 Discussion 90

3.6 References 96

$\begin{array}{lll}4.0 & \text { Chapter } 2 & 116\end{array}$ 
Apple pomace attenuates liver-adipose crosstalk and improves antioxidant status in young female rats consuming a Western diet

4.1 Abstract

4.2 Introduction

4.3 Materials and Methods

4.4 Results

4.5 Discussion

4.6 References

4.7 Supplementary Material

$5.0 \quad$ Chapter 3

Caloric Substitution of Diets with Apple Pomace was Determined to be Safe for Renal and Bone Health Using a Growing Rat Model

5.1 Abstract

5.2 Introduction

5.3 Materials and Methods

5.4 Results and Discussion

5.5 References

Supplemental Material

6.0 Chapter 4

Dissertation Discussion, Conclusions, and Future Directions

6.1 Discussion and Conclusions 


\section{List of Tables}

\section{Literature Review}

Table 1.

Table 2.

Table 3.

Table 4.

\section{Chapter 1}

Table 1.

Table 2.

Table 3.

Table 4.

Table 5.

Table 6.

\section{Chapter 2}

Table 1.

Table 2.

Table 3.

Supplementary Table 1.

Supplementary Table 2

\section{Chapter 3}

Table 1.

Table 2.

Table 3.

Table 4.

Table 5.

Table 6. 


\section{List of Figures}

\section{Literature Review}

Figure 1.

Figure 2.

Chapter 1

Figure 1.

Figure 2.

Figure 3.

Chapter 2

Figure 1.

Figure 2.

Figure 3.

Figure 4.

Figure 5.

Chapter 3

Figure 1.

Figure 2. 


\section{Introduction}

Apples are one of the most popularly consumed fruits in the United States (U.S.). Most apples produced in the U.S. are processed for apple products, such as: apple juices, ciders, and more [1]. Apple processing separates the juice from the insoluble portions of the apple, including: the skin, stem, seeds, calyx, and pulp. This insoluble apple portion is known as apple pomace and is typically regarded as "waste [2]." Additionally, apple pomace can result in rapid spoilage and environmental pollution if not swiftly managed, but proper disposal is costly [2]. Currently, it is estimated the U.S. spends $\$ 10$ million annually on apple pomace disposal and is expected to rise as the apple processing industry continues to expand [2-4]. However, studies have shown apple pomace is rich in nutrients associated with positive health outcomes $[2,5,6]$. Repurposing apple pomace for human consumption could decrease disposal costs and environmental pollution while providing nutritional value and health benefits.

Apple pomace is rich in several nutrients, including vitamins and minerals, but is particularly high in dietary fiber and phytochemicals $[2,3,7]$. Dietary fiber has been shown to have numerous health benefits, including lowering the risk for metabolic disorders such as nonalcoholic fatty liver disease (NAFLD) [8]. Phytochemicals, phenolics and flavonoids, have also been shown to play a role in NAFLD $[7,9,10]$. A synergistic relationship between dietary fiber and antioxidants exists, as dietary fiber facilitates transport of antioxidants through the intestines $[11,12]$. Given apple pomace is high in dietary fiber and antioxidants, and the synergistic function of the nutrients, whole apple pomace has potential to be repurposed for human consumption [2].

NAFLD is the most prevalent liver disease worldwide, estimated to effect over $25 \%$ of the U.S. population [13]. Additionally, it is estimated estimated $7 \%$ of healthy children and $34 \%$ of obese children have NAFLD, suggesting prevalence will increase [14]. NAFLD is defined as increased hepatic steatosis which is benign [15]. However, NAFLD can progress to the more severe non-alcoholic steatohepatitis (NASH). NASH is defined as increased hepatic steatosis in 
conjunction with inflammation of the liver [16]. An estimated $25 \%$ of individuals with NAFLD will progress to NASH, which increases the risk for liver fibrosis, cirrhosis and cancer [17].

Progression of liver disease is suggested to be a multiple-hit pathogenesis [18]. Diets high in saturated fatty acids and simple carbohydrates stimulate de novo lipogenesis (DNL) and free fatty acid (FFA) release from the adipose tissue, resulting in NAFLD [19]. Increases in DNL and FFA result in increased inflammatory cytokine production and oxidative stress generation, signifying progression to NASH [20]. Recommended treatment for NAFLD and NASH is to reduce dietary intake of simple sugars and saturated fats and to increase dietary fiber, antioxidants, and complex carbohydrates [21-23]. Apple pomace's high dietary fiber and polyphenol contents suggests potential for its utilization as a therapeutic aid for NAFLD and $\mathrm{NASH}$. Further highlighting the need for discovering potential nutrition-based treatments for the disease, there are currently no approved Food and Administration (FDA) medication for NAFLD and NASH $[24,25]$.

Despite a favorable nutrient composition as a potential dietary aid for NAFLD and NASH, apple pomace also contains fructose [2]. Fructose has been reported to promote progression of NAFLD to NASH and to be detrimental to bone and kidney health [26-28]. Therefore, it is also necessary to evaluate the safety of apple pomace. Investigating the potential of apple pomace to be utilized as a safe and sustainable food source for human consumption would not only play a role in improving health, but also reduces environmental pollution and food waste [2,4]. 


\section{References}

1. USDA ERS - Food Availability and Consumption. USDA Economic Research Service . https://www.ers.usda.gov/data-products/ag-and-food-statistics-charting-the-essentials/foodavailability-and-consumption/. Published 2017. Accessed June 1, 2018.

2. Bhushan S, Kalia K, Sharma M, Singh B, Ahuja PS. Processing of apple pomace for bioactive molecules. Crit Rev Biotechnol. 2008;28(4):285-296.

doi:10.1080/07388550802368895

3. U.S. Apple Association. Apple Industry Statistics. http://usapple.org/all-aboutapples/apple-industry-statistics/. Published 2017. Accessed January 4, 2018.

4. Kaushal NK, Joshi VK, Sharma RC. Effect of Stage of Apple Pomace Collection and the Treatment on the Physico-Chemical and Sensory Qualities of Pomace Papad (Fruit Cloth). Vol 39.; 2002. http://jglobal.jst.go.jp/en/public/20090422/200902173246576860.

5. Lu Y, Foo L. Antioxidant and radical scavenging activities of polyphenols from apple pomace. Food Chem. 2000;68(1):81-85. doi:10.1016/S0308-8146(99)00167-3

6. Gazalli $\mathrm{H}$, Malik $\mathrm{AH}$, Sofi $\mathrm{AH}$, et al. Nutritional value and physiological effect of apple pomace. Int J Food Nutr Saf. 2014;5(1):11-15.

7. Boyer J, Liu RH. Apple phytochemicals and their health benefits. Nutr J. 2004;3(1):5.

8. Anderson JW, Baird P, Davis Jr RH, et al. Health benefits of dietary fiber. Nutr Rev. 2009;67(4):188-205. doi:10.1111/j.1753-4887.2009.00189.x

9. Chang CY, Argo CK, Al-Osaimi AMS, Caldwell SH. Therapy of NAFLD: antioxidants and cytoprotective agents. J Clin Gastroenterol. 2006;40 Suppl 1:S51-60.

doi:10.1097/01.mcg.0000168648.79034.67 
10. Dreher ML. Dietary Patterns, Foods, Nutrients and Phytochemicals in Non-Alcoholic Fatty Liver Disease. In: Dietary Patterns and Whole Plant Foods in Aging and Disease. Cham: Springer International Publishing; 2018:291-311. doi:10.1007/978-3-319-59180-3_10

11. Liu RH. Whole grain phytochemicals and health. J Cereal Sci. 2007;46(3):207-219. doi:10.1016/J.JCS.2007.06.010

12. Saura-Calixto F. Dietary Fiber as a Carrier of Dietary Antioxidants: An Essential Physiological Function. J Agric Food Chem. 2011;59(1):43-49. doi:10.1021/jf1036596

13. Younossi ZM, Stepanova M, Afendy M, et al. Changes in the Prevalence of the Most Common Causes of Chronic Liver Diseases in the United States From 1988 to 2008. Clin Gastroenterol Hepatol. 2011;9(6):524-530.e1. doi:10.1016/J.CGH.2011.03.020

14. Anderson EL, Howe LD, Jones HE, Higgins JPT, Lawlor DA, Fraser A. The prevalence of non-alcoholic fatty liver disease in children and adolescents: a systematic review and metaanalysis. Wong V, ed. PLoS One. 2015;10(10):e0140908. doi:10.1371/journal.pone.0140908

15. Loomba R, Sanyal AJ. The global NAFLD epidemic. Nat Rev Gastroenterol Hepatol. 2013;10(11):686-690. doi:10.1038/nrgastro.2013.171

16. Suzuki A, Diehl AM. Nonalcoholic Steatohepatitis. Annu Rev Med. 2017;68(1):85-98. doi:10.1146/annurev-med-051215-031109

17. Michelotti GA, Machado M V., Diehl AM. NAFLD, NASH and liver cancer. Nat Rev Gastroenterol Hepatol. 2013;10(11):656-665. doi:10.1038/nrgastro.2013.183

18. Buzzetti E, Pinzani M, Tsochatzis EA. The multiple-hit pathogenesis of non-alcoholic fatty liver disease (NAFLD). Metabolism. 2016;65(8):1038-1048.

doi:10.1016/J.METABOL.2015.12.012 
19. Byrne CD, Targher G. NAFLD: A multisystem disease. J Hepatol. 2015;62(1):S47-S64. doi:10.1016/J.JHEP.2014.12.012

20. Koch LK, Yeh MM. Nonalcoholic fatty liver disease (NAFLD): Diagnosis, pitfalls, and staging. Ann Diagn Pathol. 2018;37:83-90. doi:10.1016/J.ANNDIAGPATH.2018.09.009

21. Romero-Gómez M, Zelber-Sagi S, Trenell M. Treatment of NAFLD with diet, physical activity and exercise. J Hepatol. 2017;67(4):829-846. doi:10.1016/J.JHEP.2017.05.016

22. Munteanu MA, Nagy GA, Mircea PA. Current management of NAFLD. Clujul Med. 2016;89(1):19-23. doi:10.15386/cjmed-539

23. Trappoliere M, Tuccillo C, Federico A, et al. The treatment of NAFLD. Eur Rev Med Pharmacol Sci. 2005;9(5):299-304. http://www.ncbi.nlm.nih.gov/pubmed/16231594. Accessed January 25, 2019.

24. Tilg $\mathrm{H}$, Moschen A. Weight loss: cornerstone in the treatment of non-alcoholic fatty liver disease. Minerva Gastroenterol Dietol. 2010;56(2):159-167.

http://www.ncbi.nlm.nih.gov/pubmed/20485253. Accessed January 25, 2019.

25. Diehl AM, Day C. Cause, Pathogenesis, and Treatment of Nonalcoholic Steatohepatitis. Longo DL, ed. N Engl J Med. 2017;377(21):2063-2072. doi:10.1056/NEJMra1503519

26. Odermatt A. The Western-style diet: a major risk factor for impaired kidney function and chronic kidney disease Odermatt A. The Western-style diet: a major risk factor for impaired kidney function and chronic kidney disease The Western-Style Diet and Comparison with Other Diets. Am J Physiol Ren Physiol. 2011;301:919-931.

27. Tsanzi E, Light HR, Tou JC. The effect of feeding different sugar-sweetened beverages to growing female Sprague-Dawley rats on bone mass and strength. Bone. 2008;42(5):960968. doi:10.1016/J.BONE.2008.01.020 
28. Lim JS, Mietus-Snyder M, Valente A, Schwarz J-M, Lustig RH. The role of fructose in the pathogenesis of NAFLD and the metabolic syndrome. Nat Rev Gastroenterol Hepatol.

2010;7(5):251-264. doi:10.1038/nrgastro.2010.41 
1.0.1 A Comprehensive Analysis of the Composition, Health Benefits, and Safety of Apple Pomace

R. Chris Skinner ${ }^{1}$, Joseph C. Gigliotti ${ }^{2}$, Kang-Mo Ku${ }^{3}$, Janet C. Tou ${ }^{1}$

${ }^{1}$ Division of Animal and Nutritional Sciences, West Virginia University, Morgantown, WV 26506, United States; ${ }^{2}$ Department of Integrative Physiology and Pharmacology, Liberty University College of Osteopathic Medicine, Liberty, VA, 24515, United States; ${ }^{3}$ Department of Plant and Soil Sciences, West Virginia University, Morgantown, WV 26506, United States

Corresponding Author:

Janet C. Tou, PhD

Division of Animal and Nutritional Sciences

West Virginia University

Morgantown, WV 26506

Tel: (304)293-1919

Fax: (304)293-2232

e-mail: janet.tou@mail.wvu.edu

Published in Nutrition Reviews in December 2018 as: Skinner RC, Gigliotti JC, Ku K-M, Tou JC. A comprehensive analysis of the composition, health benefits, and safety of apple pomace. Nutr Rev. August 2018. doi:10.1093/nutrit/nuy033 
1.0.1 Abstract

Apple processing results in peel, stem, seeds, and pulp being left as a waste product known as apple pomace. This review comprehensively assessed apple pomace composition for nutritional value and bioactive substances, as well as evaluated potential health benefits, and safety. Apple pomace is a rich source of health benefiting nutrients including: minerals, dietary fiber, antioxidants, and ursolic acid, suggesting its potential use as a dietary supplement, functional food, and/or food additive. Preclinical studies have found apple pomace as well as its isolated extracts to have several health benefits including; improved lipid metabolism, antioxidant status, gastrointestinal function, and metabolic disorders (e.g. hyperglycemia, insulin resistance, etc.). Safety studies have shown apple pomace to be a safe livestock feed additive and pesticide concentrations to be within safety thresholds established for human consumption. Despite promising evidence, commercial development of apple pomace for human consumption requires more research focusing on standardized methods of nutrient reporting, mechanistic studies, and human clinical trials.

Keywords: apple pomace, fiber, antioxidants, health benefits, safety 


\subsubsection{Introduction}

Diets high in fruits and vegetables are widely recommended for health benefits. The Dietary Guidelines for Americans recommends fruits and vegetables make-up one-half of your plate [1]. Globally, apples are among the most popular and frequently consumed fruits. This is due to public perception that apples are a healthy food and the availability of apples throughout the year in a variety of forms [2]. The United States (U.S.) produces 240 million bushels of apples, annually. Approximately 33\% ( 79 million bushels) of apples harvested are processed into juices, ciders, alcoholic beverages, sauces, canned, dried, and frozen apple slices [3]. However, processing of apples results in $25 \%$ of the apple mass (e.g. skin, stem, seeds, and pulp) being discarded as waste that is referred to as apple pomace [4-6].

Management of apple pomace is a major public health issue as it easily ferments making it a cause of environmental pollution. Disposal of apple pomace is costly with the U.S. spending $\$ 10$ million, annually $[5,6]$. Therefore, the development of potential commercial applications for apple pomace is a growing field of research interest. Currently, apple pomace is used as a livestock feed ingredient, for specific nutrient (dietary fiber, polyphenol, etc.) extraction for dietary supplements, and food ingredient substitute [7-9]. Given the potential nutrient value, pollution and financial demands of apple pomace disposal, an economical solution is to utilize apple pomace as a dietary supplement, functional food and/or food additive for human consumption. Therefore, the aim of this review is to address the nutritional value, health benefits, and safety of apple pomace in order to determine its potential for human consumption.

\subsubsection{Nutrient Composition of Apple Pomace}

In this review, nutrient values are reported as fresh weight (FW) rather than dry weight since apples and apple products are typically consumed "fresh." However, Lavelli and Corti [10] suggested a reduction from $70-85 \%$ moisture content to $<10 \%$ to sustain apple pomace quality and storage stability. Comparisons for nutrient value between apples and apple pomace were 
based on values reported in peer-reviewed literature published in the English language and values available in the United States Department of Agriculture (USDA) data base. Nutrient compositional study limitations include small sample sizes and confounding factors that influence nutrient content such as: differences in apple cultivars and varieties, growing conditions, harvesting, processing techniques, and storage methods and storage length were not consistently reported in the study description. Future compositional studies evaluating apple pomace should provide this information and develop standards to allow for comparisons among studies for nutrient value.

\subsubsection{Macronutrients}

Diets low in dietary fat have been shown to prevent weight gain and obesity, lower risk of cardiovascular disease (CVD), cancer, and other chronic diseases [11-14]. An apple contains 0.16-0.18\% fat, while apple pomace has been reported to range between $1.1-3.6 \%$ (Table 1 ) $[9,15,16]$. Consumption of apple pomace includes seeds, which is the portion of the apple containing the majority of fatty acids, mostly as linoleic acid (18:2n-6) and oleic acid (18:1n-9) [9]. Linoleic acid is an essential fatty acid, with some studies suggesting benefits of reduced risk of atherosclerosis, improved impaired glucose tolerance, and reducing body fat deposition [1720]. Apple pomace, due to a low fat content, is not considered a rich source of these fatty acids. Additionally, apples are low in protein although the reported amount of protein in apple pomace is higher likely due to the seeds (Table 1).

Carbohydrates account for $\sim 14 \%$ of the nutrient composition of apples [15]. Apple pomace has a greater carbohydrate content compared to apples (Table 1). Sucrose content in apple pomace showed a wide range since sucrose is highly variable among apple cultivars $[15,16]$. Both apples and apple pomace contain a large percentage of total carbohydrates as fructose and glucose $[9,15]$. Higher amounts of fructose and glucose in apple pomace compared to apples are likely due to the inclusion of the sugar-containing stem and calyx (Table 
1). Among fruits popularly consumed in the U.S. apples are considered particularly concentrated in fructose [21]. Free fructose is poorly absorbed and functions similar to dietary fibers, by escaping absorption in the small intestine and being fermented in the large intestines [22].

Apples also contain complex carbohydrates such as polysaccharides. In a preclinical study by Chen, et al [23] male Kunming mice (age 45-days old, $n=18$ animals/group) were randomly assigned to be fed a high-fat diet (HFD) (45\% kcal fat) or a HFD supplemented with apple pomace polysaccharides at doses of 200,400 , or $800 \mathrm{mg} / \mathrm{kg} \mathrm{bwt} / \mathrm{d}$ for 30 days. Results showed all doses of apple pomace polysaccharides improved serum triglycerides, total cholesterol. HDL-C, insulin, and adiponectin compared to mice fed HFD. Doses of 200 and 400, but not the highest dose of $800 \mathrm{mg} / \mathrm{kg}$ bwt $/ \mathrm{d}$ apple pomace polysaccharide significantly reduced serum low density lipoprotein-cholesterol (LDL-C). The lowest dose of apple pomace polysaccharides $(200 \mathrm{mg} / \mathrm{kg}$ bwt $/ \mathrm{d})$ showed lower $(p<0.05)$ serum cholesterol, insulin, and adiponectin compared to HFD fed mice. Additionally, $200 \mathrm{mg} / \mathrm{kg}$ bwt/d apple pomace polysaccharides reduced $(p<0.001)$ serum leptin compared to HFD fed mice. All doses of apple pomace polysaccharides increased serum hexokinase and glucagon concentrations, indicating a return to metabolic balance. Additionally, apple pomace polysaccharides supplementation to rats fed a HFD restored antioxidant capacity to control levels in a dose-dependent manner. Furthermore, all doses of apple pomace polysaccharides reduced $(p<0.05)$ fasting serum insulin and liver lipid content compared to mice fed HFD (Table 3) [23-34]. Collectively, study results showed mice fed HFD supplemented with apple pomace polysaccharides had improved insulin, glucose metabolism, lipid profile, and antioxidant status. Based on preclinical studies, apple pomace polysaccharides supplementation may be a potential treatment for diet-induced metabolic or obesity-related diseases. However, clinical studies are needed to confirm health benefits for humans.

\section{Dietary Fiber}


It has been recommended the American population increase their dietary fiber consumption for various health benefits [15]. However, only about $5 \%$ of the U.S. population achieves the recommended level of dietary fiber consumption [35]. Among the top consumed fruits in the U.S., apples with skin had one of the highest dietary fiber contents [22]. Since apple pomace includes the skin, stem, seeds, and calyx, there is a higher fiber content in apple pomace than an apple. Apple pomace has been reported to contain between 4.4-47.3 g/100g of fiber [9]. The variability in reported fiber content in apple pomace is likely due to use of different cultivars of apples and methods of quantifying or extracting dietary fiber. In apple pomace, insoluble fiber accounts for $33.8-60.0 \%$ of total fiber with cellulose accounting for $6.7-40.4 \%$ and lignin for $14.1-18.9 \%$ [9]. Soluble fiber accounts for $13.5-14.6 \%$ of total fiber in apples. Since the majority of soluble fiber in apples is in the skin, this results in apple pomace containing a larger percentage of soluble fiber than apples [36]. In particular, apple pomace contains higher pectin than apples (Table 1). Due to its high fiber, consumption of $100 \mathrm{~g}$ of apple pomace provides approximately half of the recommended daily fiber intake.

Diets high in dietary fiber have been reported to promote gastrointestinal health and to reduce the risk for diverticular diseases and certain cancers, particularly colorectal cancer $[37,38]$. Extraction of fiber-rich colloids from apple pomace was used to investigate fiber as the specific component in apple pomace responsible for gastrointestinal benefits. Sembries, et al [24] fed young (age 8 weeks) male Wistar rats ( $n=12$ animals/group) either a standard rodent diet or a standard rodent diet supplemented with $5 \%$ apple pomace fiber-rich colloid for six weeks. Fiber-rich colloids from apple pomace increased microflora fermentation indicated by significantly higher short chain fatty acids (SCFA), acetate and propionate, in the cecum. Rats provided diet supplemented with apple pomace-rich colloid also had increased $(p<0.001)$ bile acid excretion in the feces. Additionally, consumption of fiber-rich colloids from apple pomace resulted in significant decreased weight gain in the absence of reduced food consumption 
(Table 3). The authors attributed this to increased food passage rate in the gut due to the high fiber content of the apple pomace.

High dietary fiber consumption has also been linked to reduced risk of CVD [39]. Fiberrich colloids isolated from apple pomace was used to investigate fiber as a specific component in apple pomace responsible for CVD health benefits [25]. Apple pomace was mixed with hot water, and colloids were extracted from the apple pomace juice mixture. Young male Wistar rats (age 6 weeks) were randomly assigned ( $n=10$ animals/group) to be fed a standard rodent diet containing ( $5 \%$ diet weight) fiber-rich colloids isolated from different apple pomace extraction juice from different apple varieties (Boskoop, Werder Frucht, Glindow, Germany). After six weeks, feeding apple pomace fiber-rich colloids significantly reduced serum total cholesterol and low density LDL-C, while increasing serum high density lipoprotein-cholesterol (HDL-C). The excretion of bile acid and neutral sterols was also increased $(p<0.05)$ in rats fed fiber-rich colloids (Table 3). Dietary fiber by acting as a bile sequestrant improves serum lipids and lipoproteins [25].

The main fiber constituent found in apple pomace is pectin [40]. Apple pomace contains $10-15 \%$ pectin (dry weight) making it a good source of this insoluble fibre [41]. Pectin has been recommended to be the source of $30-50 \%$ of total daily dietary fiber intake due to its reported health benefits. Pectin has been shown to lower cholesterol absorption and to lower plasma and liver triglycerides [42]. In an in vitro study, Kumar and Chauhan [42] extracted pectin from apple pomace to evaluate it as an inhibitor of pancreatic lipase, which is the primary enzyme responsible for hydrolyzing dietary triglycerides. Pectin extracted from apple pomace resulted in a $94.3 \%$ inhibition of pancreatic lipase indicating purified pectin from apple pomace may be a potential anti-obesity treatment by reducing calories through inhibition of fat absorption.

\subsubsection{Micronutrients Minerals}


Micronutrients of concern in the American diet includes potassium and calcium [1]. Consumption of potassium (e.g. fruits and vegetables) lowers blood pressure [43]. According to Koutsos, et al [44] apples contain $107 \pm 2.21 \mathrm{mg}$ of potassium/100g FW. Based on a survey of popularly consumed fruits in the U.S., apples were among the fruits lowest in potassium, however apple pomace contains more potassium (Table 2) [9,15,45-49]. Calcium and phosphorus are important for bone health with adequate intake reducing risk of osteoporosis $[9,50]$. Oranges are the richest source of calcium among commonly consumed fruits in the U.S. $[22,48]$. Apples are lower but apple pomace provided more calcium than oranges (Table 2). Of popularly consumed fruits in the U.S., bananas contain the most phosphorous. Apples are lower, but when processed into pomace results in higher phosphorous content making apple pomace a richer source of phosphorus than other popularly consumed fruits in the U.S. (Table 2). The mechanisms by which bone health is improved by fruits and vegetables have not been thoroughly investigated. The acid-base hypothesis postulates acid load is buffered in part by bone tissue, leading to bone resorption and reduced bone density [51]. Fruits and vegetables are a good source of alkaline precursors such as potassium, calcium as well as magnesium. These minerals neutralize the acidic effects of low pH foods derived from the diet [52]. Apples $(5.0 \pm 0.7 \mathrm{mg} / 100 \mathrm{~g})$ provide a modest amount of magnesium with the amount in apple pomace (176 $\pm 157.5 \mathrm{mg} / 100 \mathrm{~g})$ being much higher (Table 2).

In terms of human health, food sources that increase iron consumption are of interest, since iron deficiency anemia is the most common nutrient deficiency worldwide [53]. Fruits are not considered a rich source of iron. However, the amount of iron in apple pomace was higher than in apples. Zinc deficiency is another common nutrient deficiency [54]. Zinc content is also higher in apple pomace compared to apples (Table 2). Compared to the most popularly consumed fruits in the U.S., apples only provided modest amounts of dietary minerals. On the other hand, apple pomace provides significantly more dietary minerals likely due to inclusion of peel $[9,55]$. Gorinstein, et al [56] reported apple peels to have higher amounts of sodium, 
potassium, calcium, magnesium, and iron than in whole apples. Compositional evaluation indicates potential use of apple pomace as a supplement to increase dietary mineral intake.

\section{Vitamins}

Vitamins $\mathrm{C}$ and $\mathrm{E}$ are non-enzymatic antioxidants and have been shown to be potent scavengers of reactive oxygen species (ROS). Previous studies have reported apples to be a rich source of vitamins $C$ and $E$ [57]. However, when compared to other popular consumed fruits in the U.S., apples were lower in vitamin C content $(0.057 \mathrm{mg} / \mathrm{g})$. Corrected for total antioxidant activity contributed by vitamin C, apples $(97.23 \mu \mathrm{mol}$ of vitamin C equivalents/g) ranked second after cranberries $(176.98 \mu \mathrm{mol}$ of vitamin $\mathrm{C}$ equivalents $/ \mathrm{g})$. Although apples contain less vitamin $\mathrm{C}$ than other fruits, antioxidant activity was still higher. This finding has physiological significance, as the high antioxidant bioactivity index of apples was shown to have anti-proliferative activity on cancer cells, second only to cranberries [58]. Apple pomace was reported to have $22.4 \mathrm{mg} / 100 \mathrm{~g}$ of vitamin C [59].

Vitamin $E$ is found to be abundant in seeds [57]. Apple pomace which contains seeds was reported to have $5.5 \mathrm{mg} / 100 \mathrm{~g}$ of vitamin E [59]. Lu and Foo [60] analyzed the free radical scavenging abilities of apple pomace. Vitamin $\mathrm{C}(\mathrm{EC} 50=0.35)$ had the second highest free radical scavenging activity in apple pomace and vitamin $E(E C 50=0.30)$ ranked third. Phloridzin, a phytochemical, had the highest free radical scavenging activity $(E C 50=0.60)$. Phloridzin present in apple pomace is absent in other pomace sources such as pears [61]. In addition to antioxidant vitamins, apple pomace also contains phytochemicals with antioxidant properties that may reduce the risk of various diseases.

\section{Phytochemicals}

Diets high in phytochemicals decrease risk of diseases associated with oxidative stress and inflammation [62,63]. Five major polyphenolic groups have been found in apples: flavanols, 
flavonols, hydroxycinnamates, dihydrochalcones, and anthocyanins [64,65]. Some of the most popularly consumed apple varieties including: Fuji, Red Delicious, and Gala were reported to be high in phenolics and flavonoids and supports data showing apples inhibit lipid peroxidation and scavenge free radicals, ex vivo [66,67]. Total antioxidant activity of apples is reported to be $\sim 100 \mu \mathrm{mol}$ vitamin C equivalents/g fruit, which was second only to cranberries in a study of popularly consumed fruits in the U.S [66].

The processing of fruits has been found to alter nutrient composition and content of fruit constituents [68]. However, polyphenolic antioxidants present in apples are also abundant in the apple pomace. Polyphenols are predominantly located in the skin and therefore, most polyphenols remain in the pomace [69]. Polyphenolic compounds found in apple pomace include: catechin, p-Coumaric acid, caffeic acid, and ferulic acid and have been shown to have significantly higher scavenging activities than antioxidant vitamins $E$ and $C$ indicating apple pomace's potential as a source of dietary antioxidants $[9,60]$. Apple pomace also contains polyphenols: flavanols, flavonols, hydroxycinnamates, and dihydrochalcones [61]. Of the flavonoids, quercetin and its glucosides are the most abundant flavanoids in apple pomace, and have been linked to the prevention of several diseases [66].

Saucier and Waterhouse [70] isolated polyphenols from apple pomace processed from Gala apples and evaluated antioxidant activity. Results showed apple pomace polyphenols had 2 to 3 fold greater 1-diphenyl-2-picrylhydrazyl (DPPH) radical scavenging and 10 to 30 fold greater superoxide scavenging activity than vitamins C or E. In another study [71], polyphenols were isolated from five apple pomace from six common Spanish apple cultivars (Limon Montes, Meana, Durona de tresali, de la Riega, Perezosa, and Carrio). DPPH and ferric reducing power (FRAP) assays were used to determine the antioxidant capacities of apple pomace from different apple cultivars. Results showed apple pomace to be a rich source of polyphenols for all cultivars. Certain cultivars showed greater antioxidant activity. Carrio had the highest $(p<0.05)$ DPPH and FRAP values. de la Riega and Meana, Limon Montes cultivars had higher DPPH 
values compared to other cultivars. Additionally, the study found the antioxidant activity of apple pomace can be predicted by its content of phloridzin, procyanidin B2, rutin + isoquercitrin, protocatechuic acid, and hyperin. High levels of these phytochemicals indicate more antioxidant activity [71]. Apple pomace consists $95 \%$ skin, which contains a substantial amount of polyphenols, including the polyphenolic compounds: cinnamic acid, epicatechin, caffeic acid, and procyanidin $[9,60]$. Other chemical compounds found in the skin of apples may also have potential beneficial health effects.

\section{Ursolic Acid}

The cuticle forms a protective layer on the surface of the skin of the apple and is composed of two main components: cutin and wax [72]. The wax on the cuticle layer known as the epicuticular wax protects against damage caused by insects and other pathogens and has been suggested to have antioxidant properties $[73,74]$. The main epicuticular wax in apples is ursolic acid [72]. Ursolic acid exists as several isomers including oleanolic acid [75]. Both ursolic and oleanolic acid have been reported to have antioxidant, anti-inflammatory, anti-cancer, and anti-hepatotoxic activities [76-82].

Apple pomace has been proposed to be a good source of ursolic acid [83,84]. Frighetto, et al [75] measured ursolic acid content in the skin of different apple cultivars: Fuji, Gala, Smith, and Granny Smith. Results found Smith apples had the highest content of ursolic acid (0.82 $\mathrm{mg} / \mathrm{cm} 2)$ followed by Fuji $(0.77 \mathrm{mg} / \mathrm{cm} 2)$, Granny Smith apples $(0.49 \mathrm{mg} / \mathrm{cm} 2)$, and Gala $(0.21$ $\mathrm{mg} / \mathrm{cm} 2$ ). The authors concluded apple pomace could have potential benefits since apple pomace includes the entire skin of the apple, and therefore, would be expected to provide large amounts of ursolic acid. Grigoras, et al [85] evaluated ursolic acid isomers in apple pomace processed from four apple cultivars: Gala variety Royal Gala Tenroy, Golden variety Golden, Granny Smith, and Pink Lady variety Cripps Pink. Identification using high-performance liquid chromatography (HPLC) showed apple pomace contained ursolic acid, and oleanolic acid, 
which was consistent with finding reported in apples. Furthermore, apple pomace from Gala apples was shown to have readily extractable ursolic acid [86]. The presence of ursolic acid in apple pomace provides further evidence in support of health benefits of apple pomace for human consumption.

Overall, compositional analysis of apple pomace found several compounds present in high concentration with beneficial effects that including attenuating metabolic dysfunction and oxidative stress. This provides evidence in support of commercial development of compounds isolated from apple pomace such as: total dietary fibers, pectin, and apple pomace polysaccharides as a dietary supplement, functional food and/or food additive for human consumption. Currently, extracted isolated compounds from apple pomace are being used for human dietary supplements. However, extraction and purification of specific apple pomace ingredients can be technologically challenging and costly. Also, the combination of compounds in apple pomace may have synergistic effects. Therefore, the health benefits of whole apple pomace consumption is reviewed in the next section.

\subsubsection{Health Benefits of Apple Pomace Consumption}

Ravn-Haren, et al [26] compared plasma lipid profiles of individuals consuming apple pomace, apples or apple juice. Subjects were healthy non-smoking, non-obese men and women (age 18-69 years, $n=34$ ) who did not use vitamin or mineral supplements. The experimental design was a randomized single-blinded $5 \times 4$ weeks crossover study consisting of five diet treatments, including: a restricted diet period low in pectin and polyphenols, and four periods where the restricted diet was supplemented with either, apple pomace ( $22 \mathrm{mg} / \mathrm{day})$, apples (550 g/day), cloudy (fiber containing) apple juice (500 ml/day), or clear apple juice (500 $\mathrm{ml} /$ day).

Study results showed pectin consumption was correlated $\left(r^{2}=0.983\right)$ to reduced plasma cholesterol concentration. Apple pomace consumption for four weeks had no significant effect on serum total cholesterol, LDL-C, HDL-C, and bile acid concentration. This may be attributed to 
less pectin in apple pomace ( $2.12 \mathrm{~g} /$ day $)$ than apples $(2.87 \mathrm{~g} /$ day $)$. Subjects consuming apple pomace reported a trend $(p<0.066)$ for decreased heart rate, blood pressure, serum alanine amino transferase, C-reactive protein, insulin-like growth factor 1, and insulin like growth factor binding protein 3. A significant health benefit of apple pomace consumption was improved gastrointestinal health indicated by decreased $(p<0.05)$ lithocholic acid excretion (Table 3$)$. Consumption of clear apple juice, which lacks dietary fiber, had no significant effect on gastrointestinal function. Therefore, authors suggested the fiber (pectin) content of apple pomace was the component responsible for improved intestinal health. However, the study included subjects that ranged widely in age, inconsistent adherence to the study's dietary guidelines, the apple pomace group consumed the least fructose despite higher fructose content. Additionally, lower pectin content was reported when most studies found significantly higher pectin content in apple pomace compared to apples (Table 1).

In a further analysis of the Ravn-Haren, et al [26] study, Rago, et al [27] performed untargeted metabolomics on plasma collected from subjects. Apple pomace consumption decreased aromatic amino acids, which has been associated with gut microbial fermentation and to insulin sensitivity. Additionally, apple pomace decreased plasma medium- and shortchain acylcarnitines, primary bile acids, deydropiandrosterone sulphate, and lysophospholipids, which are associated with cholesterol transport from the liver. The authors concluded apple pomace consumption can benefit cholesterol levels, insulin sensitivity, and gut microbial functionality. However, the study also found apple pomace consumption increased plasma uric acid (Table 3). Caliceti et al [87] reported a direct relationship between fructose intake and circulating levels of uric acid, which is the final product of purine metabolism. Recent preclinical and clinical evidence suggests that chronic hyperuricemia is an independent risk factor for hypertension, metabolic syndrome, and cardiovascular disease [88-90]. It is also potentially an independent risk factor for chronic kidney disease, type 2 diabetes, and cognitive decline [90- 
92]. Since fructose content is higher in apple pomace than apples (Table 1), health implications of fructose content in apple pomace should to be further investigated.

Few studies have investigated the health benefits of apple pomace consumption by humans despite preclinical studies reporting health benefits on lipid metabolism, body weight, gut health, and glucose regulation as well as on antioxidant activity [9]. Cho, et al [28] investigated the effect of feeding rats a HFD supplemented with apple pomace on body weight and circulating lipids and lipoproteins. Weanling male Sprague-Dawley rats were randomly assigned ( $\mathrm{n}=8$ animals/group) to diet groups including: HFD (15\% by diet weight, consisting of $8 \%$ lard and $7 \%$ soybean oil) to induce obesity, a HFD supplemented with $10 \%$ (diet weight) apple pomace, or a standard rodent diet. At the end of nine week feeding study, results showed rats fed HFD supplemented with $10 \%$ apple pomace significantly reduced body weight and body fat percentage, and improved serum lipid profiles indicated by lower $(p<0.05)$ serum LDL-C and higher $(p<0.05)$ serum HDL-C compared to rats fed HFD. The authors concluded reduced body weight and improved serum lipid profile in rats fed HFD supplemented with 10\% apple pomace was due to altered lipid metabolism indicated by reduced liver cholesterol and triglyceride content, and higher fecal total cholesterol and triglyceride excretion $(p<0.05)($ Table 3$)$. Significantly higher fecal lipid excretion and improved lipid absorption and metabolism in rats fed HFD supplemented with $10 \%$ apple pomace was attributed to higher fiber intake.

Bobek et al [29] reported similar effects of improved lipid profile. Weanling male Wistar rats were randomly assigned ( $n=20$ rats/group) to be fed either a cholesterol diet $(0.3 \%$ diet weight) or a cholesterol diet supplemented with $5 \%$ (diet weight) apple pomace for 10 weeks. Results showed rats fed cholesterol diet supplemented with $5 \%$ apple pomace reduced $(p<0.05)$ liver cholesterol content by $11 \%$ compared to rats fed cholesterol diet. Rats fed diet supplemented with apple pomace reduced $(p<0.05)$ liver HMG-CoA reductase activity (a rate controlling enzyme for de novo cholesterol synthesis), reduced plasma levels of conjugated dienes, and increased $(p<0.01)$ the fractional catabolic rate of plasma cholesterol, but did not 
significantly reduce plasma total cholesterol (Table 3). These results were attributed to the ability of fiber to bind to bile acids. In addition, antioxidant status was determined. Cholesterol diets supplemented with apple pomace significantly reduced antioxidant enzymes: SOD, catalase, and GPx activity in erythrocytes (Table 3). The authors suggested decreased erythrocyte antioxidant enzyme activity was due to an increase in vitamins $C, E$, and $A$, as well as $\beta$-carotene, retinol, phytochemicals, and flavonoids from consumption of the apple pomace. Based on the results the authors concluded apple pomace plays a significant role in antioxidant defense systems. However, the study did not support the proposed mechanism by measuring antioxidant vitamins or phytochemical content of apple pomace.

A preclinical study investigated polyphenol and fiber rich apple pomace on antioxidant status and gastrointestinal physiology. Juskiewicz, et al [30] conducted a feeding study using unprocessed apple pomace and apple pomace in which the polyphenol content was significantly reduced by ethanol extraction, referred to as processed apple pomace. Male Wistar rats (age 35 days) were randomly assigned ( $n=8$ animals/group) into three groups consisting of a standard rodent diet (control) or a standard control diet supplemented with either 15\% (diet weight) unprocessed apple pomace or $14 \%$ (diet weight) processed apple pomace. Results showed erythrocyte SOD was significantly higher in unprocessed apple pomace. Both apple pomace groups significantly increased serum antioxidant capacity of water-soluble substances compared to control. However, only unprocessed apple pomace significantly increased serum antioxidant capacity of lipid-soluble substances. Additionally, unprocessed apple pomace decreased $(p<0.05)$ liver thiobarbituric acid reactive substances (TBARS) compared to control (Table 3). As expected, unprocessed apple pomace which is rich in polyphenols showed greater improvements in antioxidant status.

The effect of apple pomace on gastrointestinal health was determined by measuring digestibility and gastrointestinal function markers. Nitrogen was used as a measure of digestibility of the apple pomaces by measuring nitrogen intake compared to nitrogen excreted 
in the feces and urine. Nitrogen intake did not differ among groups, but both apple pomace groups decreased $(p<0.05)$ fecal nitrogen compared to control. Only unprocessed apple pomace significantly decreased nitrogen in the urine. Nitrogen digestibility was significantly decreased in both apple pomace groups, but nitrogen utilization was not altered. Both apple pomace groups significantly increased cecum tissue weight, digesta, dry matter percentage, $\mathrm{pH}$, and ammonia in the cecum compared to control. Unprocessed apple pomace significantly decreased digesta $\beta$-glucuronidase, an enzyme catalyzing the breakdown of complex carbohydrates, compared to control while processed apple pomace showed greater microbiota glycolytic activity indicated by significantly decreased digesta $\beta$-glucuronidase and $\beta$ glucosidase. Rats fed unprocessed apple pomace also had the lowest $(p<0.05)$ digesta $\mathrm{pH}$ in the colon. Both apple pomace groups promoted fermentation indicated by significantly decreased fecal $\mathrm{pH}$ and higher cecal digesta SCFA compared to control. Although, only processed apple pomace decreased fecal pH at the end of the 28 days study (Table 3). Collectively, the results suggested both unprocessed and processed apple pomace improved intestinal health through beneficial decreases in gut enzymes without significantly impacting nitrogen utilization. Also, unprocessed apple pomace favorably modified antioxidant status.

In another study examining the potential role of apple pomace on gastrointestinal health, Kosmola, et al [31] randomly assigned young (age 4 weeks) male Wistar rats $(n=8$ animals/group) to be fed standard rat diet (control) or standard rat diet supplemented with apple pomace $(0.23 \% \mathrm{w} / \mathrm{w})$, flavonoid-reduced $(0.10 \% \mathrm{w} / \mathrm{w})$ apple pomace, or flavonoid-deprived $(0.01 \% \mathrm{w} / \mathrm{w})$ apple pomace. Following four weeks, gastrointestinal fermentation and health were evaluated. Resulted showed all apple pomace groups had increased intestinal fermentation, significantly higher fecal SCFA, and decreased cecum $\mathrm{pH}$ compared to control. However, rats fed apple pomace had significantly increased cecum tissue weight compared to control indicating greater ability to metabolize energy dense foods and had the lowest $\beta$ glucuronidase which has been linked to a decreased risk for colon cancer [93]. Whereas, 
flavonoid-reduced apple pomace supplementation significantly increased glycolytic activity of cecal microbiota and beneficially modified the ratio of cecal SCFA and branched-chain fatty acids compared to control. Further, flavonoid reduction of apple pomace resulted in significantly decreased cecal ammonia and colonic pH compared to control (Table 3). The authors concluded apple pomace consumption improved gastrointestinal health with flavonoids in apple pomace showing improved local interactions in the digestive tract.

When using animal models to study digestive health, the pig has advantages of similar digestive and associated metabolic processes to humans. The pig is an omnivorous animal with comparable nutritional requirements to humans and also has a similar intestinal microbial ecosystem [94]. Sehm, et al [32] fed young (age 24 days old) male piglets (Pietráin $\times$ (Deutsche Landrasse $\times$ Deutsches Edelschwein) a standard swine diet or a standard swine diet supplemented with $3.5 \%$ (by weight) apple pomace for six weeks ( $n=39$ pigs/group). Results showed apple pomace supplementation had no significant effect on energy intake, feed uptake, and average daily weight gain. However, apple pomace increased villi breadth in the jejunum $(p<0.01)$ and ileum $(p<0.001)$ suggesting improved nutrient absorption. Apple pomace also reduced $(p<0.007)$ gut-associated lymphoid tissue (Table 3 ), which plays a role in the immune function of the gastrointestinal tract, indicating apple pomace may offer anti-inflammatory capabilities. Based on the results, the authors concluded apple pomace improved gastrointestinal health.

Apple pomace was compared to other fruit byproducts as a source of fiber with potential benefits for lipid metabolism, intestinal health, and glucose regulation. Macagnan, et al [33] compared apple pomace to two other common fruit byproducts; orange bagasse and passion fruit peel. Weanling male Wistar rats ( $n=8$ animals/group) were randomly assigned to four groups: supplemented with cellulose $(50 \mathrm{~g} / \mathrm{kg})$ as a control, apple pomace $(68.8 \mathrm{~g} / \mathrm{kg})$, orange bagasse $(99.6 \mathrm{~g} / \mathrm{kg})$, or passion fruit peels $(86.2 \mathrm{~g} / \mathrm{kg})$. Different amounts of the fruit byproducts were used to balance macronutrient and energy amounts among diets. After 34 days of feeding, 
food consumption, weight gain, and feed efficiency ratio did not differ among diet groups. All fruit byproducts had significantly higher apparent digestibility of digestive fiber compared to cellulose Apple pomace and orange bagasse had significantly increased fecal nitrogen percentage, a marker of diet digestibility, compared to passion fruit peels and control (Table 3). All fruit byproducts significantly decreased dry fecal production and increased fecal moisture content. However, none significantly altered gastrointestinal transit time. Apple pomace had no effect on intestinal fermentation, but the other fruit byproducts promoted intestinal fermentation indicated by reduced $(p<0.05)$ fecal $\mathrm{pH}$. However, apple pomace had the highest $(p<0.05)$ fecal lipid percentage. This in turn, was expected to influence circulating lipids. All fruit byproducts had significantly reduced serum triglycerides and liver LDL-C, but did not alter serum total cholesterol (Table 3). Liver fat percentage was not significantly altered by any of the diet groups.

Regarding glucose regulation, all fruit fiber groups significantly decreased area under the curve for glucose (Table 3). Orange bagasse and passion fruit peel, but not apple pomace significantly reduced postprandial fasting glucose compared to cellulose. Only orange bagasse significantly decreased glycemic peak. Other studies reported apple pomace consumption improved blood glucose. Juskiewicz, et al [30] reported unprocessed polyphenol rich apple pomace reduced serum glucose. Kosmala, et al [31] found feeding apple pomace and flavonoidreduced apple pomace, but not flavonoid-deprived apple pomace reduced serum glucose (Table 3). All food byproducts studied were similar in terms of digestibility, gastric transit, and lipid metabolism alterations. Orange bagasse appeared to be the most efficient at glycemic control, which may be related to polyphenol content, as high polyphenol foods have been shown to inhibit glucose absorption [95]. Collectively, the results indicate the importance of polyphenols rich fiber as the compound in apple pomace influencing glucose regulation.

Ma, et al [34] investigated apple pomace combined with rosemary extract, a popular flavoring herb, on fructose consumption-induced insulin resistance. Male Sprague Dawley rats (age 7 weeks) were randomly assigned into two initial groups for the first 13 weeks of the study: 
water control ( $n=6$ animals) or $10 \%(w / v)$ fructose in the drinking water $(n=27)$. Following 13 weeks, the fructose group was divided into three groups ( $n=9$ animals/group) fructose control, fructose with $100 \mathrm{mg} / \mathrm{kg}$ of apple pomace and rosemary extract, and fructose with $500 \mathrm{mg} / \mathrm{kg}$ of apple pomace and rosemary extract. At the end of five weeks, the higher dose of $500 \mathrm{mg} / \mathrm{kg}$ apple pomace and rosemary extract significantly decrease fasting plasma glucose compared to fructose control. Fasting plasma insulin, homeostasis model assessment of insulin resistance (HOMA-IR) and adipose insulin resistance (Adipo-IR) were significantly decreased at both doses of apple pomace and rosemary extract compared to fructose control. High dose (500 $\mathrm{mg} / \mathrm{kg}$ ) apple pomace and rosemary extract improved insulin resistance. Gastrocnemius sarcolemmal CD36 contributes to insulin resistance by facilitating fatty acid uptake and downregulation of glucose transporter-4 (GLUT-4). Rats fed the high dose $(500 \mathrm{mg} / \mathrm{kg})$ of apple pomace and rosemary extract significantly reduced gastrocnemius sarcolemmal CD36 and GLUT-4 stain intensity compared to fructose control (Table 3). The authors concluded high dose apple pomace and rosemary extract improved fructose consumption-induced insulin resistance by attenuation of impaired CD36 cells and GLUT-4 transporter.

Collectively, human and animal study results showed apple pomace consumption improved lipid metabolism, blood lipid profile, and metabolic dysfunction (e.g. hyperglycemia, insulin resistance) induced by unhealthy diets (e.g. high in fat, high in fructose), as well as antioxidant status and gastrointestinal health and therefore, warrants further research towards development of apple pomace for human consumption. Of the preclinical studies investigating potential health benefits of apple pomace consumption, all used animal models consisting of growing or young adult males. Most feeding studies were of short-duration (4-10 weeks). Typical of animal feeding studies, doses of apple pomace or purified apple pomace ingredients were higher than typically consumed or achievable by humans and therefore, translational dosing studies are needed. Evidence regarding the potential of health benefits of apple pomace 
for human consumption is promising. However, no studies have specifically addressing the safety of apple pomace for human consumption.

\subsubsection{Safety of Apple Pomace Consumption}

The safety of apple pomace for human consumption has not been comprehensively reviewed and few studies exist regarding potential health risks of consuming apple pomace. A commercial use for apple pomace has been as a feed additive for livestock animals (e.g. cattle and goats). Studies found apple pomace to be a suitable feed additive for livestock by providing adequate nutrients with no detriments to protein digestion, growth, pregnancy outcome, and milk production [96-99]. However, ruminants are not translational animal models and nutritional inadequacies resulting from apple pomace intake is not a major concern for humans. Issues of greater concern for apple pomace consumption by humans is presence of natural toxins in apple seed and pesticide exposure.

The cyanogenic glycoside, amygdalin is a naturally-occurring plant toxin. When apple tissues are disrupted amygdalin interact with endogenous digestive enzymes resulting in the release of hydrogen cyanide. Consumption of cyanogenic plants can result in acute cyanide poisoning with symptoms including: headaches, dizziness, hypotension, loss of consciousness, coma, and death [100-102]. Bolarinwa, et al [100] analyzed apple seed amygdalin levels in 15 varieties of apples. Results found Golden Delicious (3.91 $\pm 0.49 \mathrm{mg} / \mathrm{g})$, Royal Gala ( $2.96 \pm 0.12$ $\mathrm{mg} / \mathrm{g})$, and Red Delicious $(2.80 \pm 0.50 \mathrm{mg} / \mathrm{g}$ ) had the highest seed amygdalin content. Braeburn $(1.19 \pm 0.12 \mathrm{mg} / \mathrm{g})$ and Egremont Russet $(0.95 \pm 0.22 \mathrm{mg} / \mathrm{g})$ had the lowest seed amygdalin content (Table 4) [100,103-115]. Variations in amygdalin content can be attributed to different cultivars and environmental differences. The authors suggested that amygdalin content found in apple seeds was high and consumption of apple seeds could be a cause for concern.

Opyd, et al [103] fed male Wistar rats (age 8 weeks) apple seeds isolated from apple pomace to determine if the amygdalin content of apple seeds included in the pomace impacted 
health. Rats were randomly divided ( $n=10$ animals/group) into three groups consisting of a high saturated fat (7\% lard, 1\% cholesterol) and high fructose (68.75\%) (HSHF) diet, HSHF supplemented with $0.24 \%$ (the equivalent of $160 \mathrm{mg} / \mathrm{kg}$ ) amygdalin, or HSHF supplemented with $18.4 \%$ apple seeds (amygdalin content=0.24\%). Following 14 days, apple seed supplementation significantly reduced dietary intake and body weight compared to control and amygdalin diet groups. Additionally, protein digestibility and nitrogen retention were decreased $(p<0.001)$. Apple seed supplementation significantly increased cecum tissue mass and digesta mass, increased microbial enzyme activity, and increased $(p<0.001)$ digesta SCFA compared control and amygdalin diet groups. Serum glucose, triglycerides, and total cholesterol were not significantly affected by apple seed supplementation, but HDL-C was increased $(p=0.015)$ compared to control. Apple seed supplementation also increased ( $p=0.002)$ serum antioxidant capacity of water-soluble substances compared to control and amygdalin and significantly decreased liver TBARS compared to control. The authors concluded amygdalin content of apple seeds did not negatively impact markers of digestion, blood lipids, and improved antioxidant status of rats. Results indicated apple pomace which contain apple seeds to be safe for consumption.

The National Institutes of Health Toxicology Data Network reported the lethal dose of hydrogen cyanide to be $50-300 \mathrm{mg}$. The potential amount of hydrogen cyanide released from apple seeds is $0.6 \mathrm{mg}$ of hydrogen cyanide/g, which would require consumption of 83-500 apple seeds for acute cyanide poisoning [104]. At most, an apple can contain 10 seeds and depending on apple size $\sim 1-2$ apples are needed to produce $100 \mathrm{~g}$ of apple pomace. Therefore, an individual would need to consume nearly $800 \mathrm{~g}$ of apple pomace for potential acute cyanide poisoning [116,117].

Another potential adverse effect of apple pomace consumption by humans is pesticide exposure since apple crops are heavily sprayed with pesticides, which includes fungicides and plant growth regulators [9]. Lead arsenate pesticides were once popularly used in apple and 
other fruit orchards. Although many countries including the U.S. have ban the use of lead arsenate and arsenic-based pesticides, arsenic and lead can persist in soil for years [118]. The media has alerted consumer to presence of arsenic in food, most notably in apple juice [119]. No studies have investigated apple byproducts; however, it has been reported arsenic primarily remains in soil, roots, and leaves $[120,121]$. Additionally, food processing has been suggested to be effective for reducing pesticide residue $[122,123]$. Washing fruit and other produce has been shown to be effective [124]. Washing apples with $10 \mathrm{mg} / \mathrm{ml}$ sodium bicarbonate for 12-15 minutes reduced the pesticides phosmet and thiabendazole levels in apples by $95.6 \%$ and $80 \%$, respectively. Using bleach, a common washing method, did not effectively remove pesticides [125]. However, washing may not be sufficient for systemic pesticides, such as neonicotinoids which translocate to all parts of the plant including the fruit [126]. Neonicotinoids have been found to penetrate apple flesh with 24 hours of topical application, indicating potential for neonicotinoid residues in apple pomace [106].

To assess health risk of pesticides on humans, the Environmental Protection Agency (EPA) uses reference dose (RfD) which is an estimated measurement of daily human oral exposure to acute or chronic dose that produces no adverse short-term or lifetime health risks based on animal studies as well as \% population adjusted dose (\% PAD) which is based on RfD and adjusts for additional susceptibility (e.g. infants, children, pregnancy) with values less than 100\% PAD considered safe $[127,128]$.

Apples contained low amounts of neonicotinoids $(<0.001 \mathrm{mg} / \mathrm{kg}$ ) however, acetamiprid were more frequently detected in apples than other fruits and vegetables, based on the USDA Pesticide Data Program from 2004-2011 [106]. In a mouse study, oral ingestion of 30 $\mathrm{mg} / \mathrm{kg} /$ day of acetamiprid for 35 days resulted in significantly decreased testis, epididymis, seminal vesicle, and prostate weights compared to mice provided no acetamiprid. Histological evaluation showed acetamiprid damaged Leydig cells indicated by increased $(p<0.05)$ testicular p38 MAPK, a marker of stress and inflammation, and decreased testicular antioxidants: 
catalase, GPX and SOD. Sperm count, viability and motility, rate of intact acrosomes, and serum testosterone were significantly decreased by acetamiprid ingestion (Table 4). Based on the results acetamiprid reduced male fertility [105].

Chen, et al [106]. analyzed specific neonicotinoid residues in fruits and vegetables using liquid chromatography-mass spectrometry. Analysis of apples included several varieties, including: Cortland, Granny Smith, Fuji, Red Delicious, Golden Delicious, Gala, Honey Crisp, and Macintosh. Results found apples to have negligible amounts of most neonicotinoids with the exceptions of Granny Smith and Honey Crisp reporting high concentrations of acetamiprid of 0.0407 and $\mathrm{mg} / \mathrm{kg}$ and $0.1007 \mathrm{mg} / \mathrm{kg}$, respectively (Table 4). Based on these doses reported in apples, results indicate a low risk for acetamiprid toxicity in humans since acute RfD for acetamiprid is $0.10 \mathrm{mg} / \mathrm{kg} /$ day and the chronic $\mathrm{RfD}$ is $0.07 \mathrm{mg} / \mathrm{kg} /$ day, with \% PAD ranging from $10-40 \%$ [107]. Based on the levels of toxicity reported for neonicotinoids in the mouse study by Zhang, et al [105] $\sim 30 \mathrm{mg} / \mathrm{kg}$ of neonicotinoids would need to be consumed for acute toxicity in humans (Table 4).

Fungicides are another widely used chemical that results in better fruit yield and quality. However, fungicides are environmentally mobile through wind and rain runoff and can be toxic to humans [129]. Depending on dose, fungicides vary in adverse side effects in humans from allergies to cancer [130]. Lozowicka [108] evaluated fungicide residues in a variety of fruits using spectrophotometric and chromatographic techniques. Fungicide residue was found on $52 \%$ of 974 sampled fruits, including apples. However, $1.3 \%$ of fruits, including apples ( $n=696$ ), sampled exceeded the maximum residue level of pesticides. Dithiocarbamates, the most widespread fungicide in the world, occurred most frequently in apples. In order to assess safety, the author calculated both the long-term risk using hazard index, a measure of the maximum amount of pesticide intake and safety and acute reference dose, which estimates 24 hours daily oral exposure without long-term risk. A hazard index and an acute reference dose over 100\% indicates potential risk to the consumer. Short-term and long-term risks associated of all 
pesticides found on apples did not pose a health risk since hazard index and acute reference dose values ranged from 0 to $4.65 \%$ (Table 4). The authors concluded long-term risks associated with consuming fungicides from apples are low in children and adults based on their risk estimates of consumed pesticides compared to acceptable daily intakes.

Liu, et al [109] analyzed fungicide residue in fresh apples $(n=24)$ using HPLC. Of the apples ( $n=24)$ sampled $50 \%$ had detectable fungicide residue. Three classes of fungicides found in apples were thiophanate, carbendazim, and pyrimethanil. Liu, et al [113] reported thiophanate residues in apples were within established maximum allowed residue levels (2 $\mathrm{mg} / \mathrm{kg}$ ). Additionally, the EPA states all foods known to have carbendazim and thiophanate including apples have low \% PAD [113,131]. However, apples were found to have the highest amount of pyrimethanil among all surveyed fruits that included: grapes, watermelons, bananas, blueberries, and peaches. Since no maximum residue levels have been established for pyrimethanil and carbendazim conclusions cannot be drawn. However, the EPA's data on cyprodinil, a similar fungicide to pyrimethanil found minimal acute and chronic risk with the highest \% PAD being 5.8\% [110,113]. The authors also calculated acute and chronic risk of pesticide exposure and found negligible acute or chronic risk for thiophanate. According to the authors long-term risk from fungicide exposure from apple consumption was negligible.

Plant growth regulators are also commonly used in apple growth and orchard maintenance to defend against fruit drop and delay ripening [132]. Maiti, et al [111] studied residues of naphthaleneacetic acid, a commonly used plant growth regulator, in apples by HPLC method. Seven samples were analyzed as whole apples, apple skin only, and apple without the skin. Apple skin showed the highest levels of naphthaleneacetic acid $(0.433 \mathrm{mg} / \mathrm{kg})$. Naphthaleneacetic acid content in whole apple and apples without skin ranged from 0.042 $\mathrm{mg} / \mathrm{kg}$ to $0.285 \mathrm{mg} / \mathrm{kg}$ (Table 4). No maximum permissible concentration for humans has been established The EPA states naphthaleneacetic acid to poses no acute or chronic health risks and levels in foods so low that no measurements are necessary [112]. Liu, et al [113] analyzed 
fresh fruits in China (the country producing the most apples worldwide) for plant growth regulators residue using HPLC reported no detectable plant growth regulators residues (Table 4). Diphenylamine is a plant growth regulator commonly used to control browning during apple storage. Lozowicka [108] reported diphenylamine to be present on $14.6 \%$ of apple samples analyzed $(n=696)$, however dose was not reported. The EPA reports animal studies on diphenylamine studies have shown it to be slightly toxic through oral, dermal, and inhalation route, causing increased bladder tumors in male and female mice, as well as reticulum cell sarcomas in mice. However, the EPA has found diphenylamine to pose a low risk for toxicity, setting a tolerance level of 10ppm for apples and 30ppm for apple pomace (Table 4) [114]. The USDA has a database of pesticides detected in various foods, the highest concentration at which the pesticides were detected, and the established EPA Tolerance Level for the pesticide. The most recently published data on the USDA database is from 2015, with $n=708$ apples being sampled and tested for 223 pesticides. No pesticide was found to be over the EPA's established Tolerance Levels. These results indicate apples and in turn, apple pomace likely does not contain levels of pesticides harmful to human health [115].

Collectively, studies results showed apple pomace to be a safe feed additive for livestock and pesticide content in apples to be within accepted safe standards for human consumption. Previous studies on feeding apple pomace to rodents and humans have not shown health detriments. However, it should be noted that no studies directly evaluated potential risks associated with pesticide residues in apple pomace. Although apples contain naturally toxic substances (e.g. amygdalin), the amount of apple pomace that must be consumed to result in acute toxicity would be nearly $2 \mathrm{lbs}$. Therefore, apple pomace appears to be safe for human consumption. However, more studies are needed on the safety of apple pomace for human consumption to ensure various pesticides and harmful compounds are not retained at toxic levels in apple pomace. 


\subsubsection{Potential Food Uses}

Apple pomace is being studied for extraction and isolation of nutrients such as dietary fiber and polyphenols for use as purified food ingredients. Dietary fibers extracted from apple pomace and used as a replacement for fat in cookies resulted in changes in cookie size, shape, and color but maintained a pleasing texture for consumption [133]. Others have investigated apple pomace as an ingredient in baked goods. Apple pomace was incorporated into cakes to increase dietary fiber and polyphenol content. Cakes prepared with $25 \%$ apple pomace wheat flour blend resulted in $14.2 \%$ total dietary fiber content compared to $0.47 \%$ in wheat flour. Additionally, $25 \%$ apple pomace blend increased polyphenols by $50 \%$. Results showed incorporating apple pomace as an ingredient in cakes improved its nutritional profile [134]. Masoodi, et al [8] utilized apple pomace as a source of dietary fiber in wheat bread. Blends were made by incorporating 2, 5, 8, and $11 \%$ apple pomace into wheat flour. Sensory evaluation found up to $5 \%$ apple pomace in wheat bread maintained favorable texture, color, general appearance, taste and odor. Unrefined dried powered apple pomace was evaluated in an ingredient pie filling and oatmeal cookies. Apple pomace added at $10-20 \%$ total formula of pie filling resulted in no differences in sensory attributes except for texture. Apple pomace added to oatmeal cookies at 30,40 , and $50 \%$ weight resulted in flavor changes. However, both products were rated as moderately liked [135]. Issar, et al [136] utilized apple pomace to develop a fiberenriched yogurt. Apple pomace was added at 2.5, 5, 7.5 and $10 \%$ to whole milk then inoculated with starter culture. Fiber ranged from 2.42 to $9.94 \%$ with optimal sensory attributes of color, flavor, texture, and overall acceptability reported with 5\% apple pomace. To our knowledge no studies have evaluated apple pomace as an edible food. Apple pomace has high potential to be reconstituted into a food product due to its favorable nutritional profile and reported sweet taste and smell $[9,135,137]$. However, apple pomace would need to be dried due to its high moisture content and propensity for rapid spoilage [9]. Following drying, apple pomace could potentially 
be reconstituted into snack bars, granolas, flavoring powders, toppings, and other innovative food products.

\subsubsection{Conclusion}

Apple pomace is a byproduct of the apple processing industry that is typically regarded as waste and presents a public health issue as its disposal is difficult and costly. As reviewed in this paper, nutrient composition of apple pomace showed higher content of dietary fiber, essential fatty acid, protein, several dietary minerals important to human health, several classes of dietary antioxidants, and ursolic acid as compared to apples and other popularly consumed fruits in the U.S., making it a potentially nutritious for human consumption.

Collectively, preclinical study results reported health benefits of improved lipid metabolism, antioxidant capacity, and digestive health indicating apple pomace's potential as a dietary supplement, food additive or functional food for human consumption. Studies have shown no deleterious effects in livestock from consumption of apple pomace as a feed additive and research on pesticide content in apples has shown safe levels for short- and long-term human consumption. However, further mechanistic, translational dose, and clinical studies on both health benefits as well as safety of apple pomace for human consumption are required. Apple processing is a continually growing industry, as ciders and juices are in high demand, resulting in apple pomace being readily available. Therefore, re-purposing apple pomace as a commercial product for human consumption can result in an environmental and economical solution to apple waste generated by industrial processing of apples.

\section{Acknowledgements}

Dean's funding support by Dean Daniel J. Robison, West Virginia University Davis College of Agriculture, Natural Resources, and Design and the West Virginia University Office of the Provost Fellowship. 
Author Contributions

R. Chris Skinner and Janet Tou initiated this review and conducted the literature search, screened and selected articles, and wrote the paper. R. Chris Skinner finalized the manuscript. All authors participated in critical review, revision, and approval of the manuscript.

Conflict of Interest

The authors declare no conflicts of interest. 


\subsubsection{References}

1. Department of Health and Human Services. Dietary Guidelines for Americans 2015-

2020 - U.S. Department of Health and Human Services, U.S. Department of Agriculture. New York NY: Skyhorse Publishing; 2017.

2. Koutsos A, Tuohy K, Lovegrove J. Apples and cardiovascular health-is the gut microbiota a core consideration? Nutrients. 2015;7(6):3959-3998.

3. U.S. Apple Association. Apple Industry Statistics. http://usapple.org/all-aboutapples/apple-industry-statistics/. Published 2017. Accessed January 4, 2018.

4. Wang HJ, Thomas RL. Direct use of apple pomace in bakery products. J Food Sci. $1989 ; 54(3): 618-620$.

5. Shah G, Masoodi FA. Studies on the utilization of wastes from apple processing plants. Indian Food Pack. 1994;48(5):47-52.

6. Kaushal N, Joshi V, Vaidya D. Effect of stage of apple pomace collection and the treatment on the physico-chemical and sensory qualities of pomace Papad (fruit cloth). Indian Food Pack. 2001;55(5):64-69. http://jglobal.jst.go.jp/en/public/20090422/200902173246576860. Accessed January 10, 2018.

7. Masoodi F, Sharma B, Chauhan GS. Use of apple pomace as a source of dietary fiber in cakes. Plant Foods Hum Nutr. 2002;57(2):121-128.

8. Masoodi FA, Chauhan GS. Use of apple pomace as a source of dietary fiber in wheat bread. J Food Process Preserv. 1998;22(4):255-263.

9. Bhushan S, Kalia K, Sharma M, Singh B, Ahuja PS. Processing of apple pomace for bioactive molecules. Crit Rev Biotechnol. 2008;28(4):285-296.

doi:10.1080/07388550802368895

10. Lavelli V, Corti S. Phloridzin and other phytochemicals in apple pomace: Stability evaluation upon dehydration and storage of dried product. Food Chem. 2011;129(4):1578-1583. 
11. Astrup A, Grunwald G, Melanson E, Saris W, Hill J. The role of low-fat diets in body weight control: a meta-analysis of ad libitum dietary intervention studies. Int J Obes. 2000;24(12):1545-1552.

12. Lichtenstein $A H$, Van Horn L, Brands M, et al. Very low fat diets. Circulation. 1998;98(9):935-939. http://www.ncbi.nlm.nih.gov/pubmed/9738652. Accessed January 8, 2018. 13. Astrup A, Ryan L, Grunwald GK, et al. The role of dietary fat in body fatness: evidence from a preliminary meta-analysis of ad libitum low-fat dietary intervention studies. Br J Nutr. 2000;83(S1):S25-S32.

14. Howard B V, Van Horn L, Hsia J, et al. Low-fat dietary pattern and risk of cardiovascular disease. JAMA J Am Med Assoc. 2006;295(6):655-666.

15. United States Department of Agriculture. Food Composition Databases Show Foods -Apples, raw, with skin. https://ndb.nal.usda.gov/ndb/foods/show/2122. Published 2016. Accessed January 24, 2018.

16. Queji MD, Wosiacki G, Cordeiro GA, Peralta-Zamora PG, Nagata N. Determination of simple sugars, malic acid and total phenolic compounds in apple pomace by infrared spectroscopy and PLSR. Int J Food Sci Technol. 2010;45(3):602-609.

17. Nicolosi RJ, Rogers EJ, Kritchevsky D, Scimeca JA, Huth PJ. Dietary conjugated linoleic acid reduces plasma lipoproteins and early aortic atherosclerosis in hypercholesterolemic hamsters. Artery. 1997;22(5):266-277.

18. Houseknecht KL, Heuvel JPV, Moya-Camarena SY, et al. Dietary conjugated linoleic acid normalizes impaired glucose tolerance in the Zucker diabetic fatty fa/fa rat. Biochem Biophys Res Commun. 1998;244(3):678-682.

19. Rainer L, Heiss CJ. Conjugated linoleic acid: health implications and effects on body composition. J Am Diet Assoc. 2004;104(6):963-968. doi:10.1016/J.JADA.2004.03.016 
20. Park Y, Storkson JM, Albright KJ, Liu W, Pariza MW. Evidence that the trans-10,cis-12 isomer of conjugated linoleic acid induces body composition changes in mice. Lipids. 1999;34(3):235-241.

21. Southgate D, Paul AA, Dean AC, Christie AA. Free sugars in foods. Int J Food Sci Nutr. 1978;32(5):335-347. http://www.ncbi.nlm.nih.gov/pubmed/363937. Accessed January 24, 2018. 22. Slavin JL, Lloyd B. Health benefits of fruits and vegetables. Adv Nutr. 2012;3(4):506516.

23. Chen L, Liu L, Li C, et al. A mix of apple pomace polysaccharide improves mitochondrial function and reduces oxidative stress in the liver of high-fat diet-induced obese mice. Mol Nutr Food Res. 2017;61(3). doi:10.1002/mnfr.201600433

24. Sembries S, Dongowski G, Mehrländer K, Will F, Helmut D. Physiological effects of extraction juices from apple, grape, and red beet pomaces in rats. J Agric Food Chem. 2006;54(26):10269-10280.

25. Sembries S, Dongowski G, Mehrländer K, Will F, Dietrich H. Dietary fiber-rich colloids from apple pomace extraction juices do not affect food intake and blood serum lipid levels, but enhance fecal excretion of steroids in rats. J Nutr Biochem. 2004;15(5):296-302. doi:10.1016/J.JNUTBIO.2003.12.005

26. Ravn-Haren G, Dragsted LO, Buch-Andersen T, et al. Intake of whole apples or clear apple juice has contrasting effects on plasma lipids in healthy volunteers. Eur J Nutr. 2013;52(8):1875-1889.

27. Rago D, Gürdeniz G, Ravn-Haren G, Dragsted LO. An explorative study of the effect of apple and apple products on the human plasma metabolome investigated by LC-MS profiling. Metabolomics. 2015;11(1):27-39.

28. Cho KD, Han CK, Lee BH. Loss of body weight and fat and improved lipid profiles in obese rats Fed apple pomace or apple juice concentrate. J Med Food. 2013;16(9):823-830. doi:10.1089/jmf.2013.2784 
29. Bobek P, Ozdín L, Hromadová M. The effect of dried tomato, grape and apple pomace on the cholesterol metabolism and antioxidative enzymatic system in rats with hypercholesterolemia. Mol Nutr Food Res. 1998;42(5):317-320. doi:10.1002/(SICI)15213803(199810)42:05<317::AID-FOOD317>3.0.CO;2-Y

30. Juśkiewicz J, Żary-Sikorska E, Zduńczyk Z, Król B, Jarosławska J, Jurgoński A. Effect of dietary supplementation with unprocessed and ethanol-extracted apple pomaces on caecal fermentation, antioxidant and blood biomarkers in rats. Br J Nutr. 2012;107(8):1138-1146. doi:10.1017/S0007114511004144

31. Kosmala M, Kołodziejczyk K, Zduńczyk Z, Juśkiewicz J, Boros D. Chemical composition of natural and polyphenol-free apple pomace and the effect of this dietary ingredient on intestinal fermentation and serum lipid parameters in rats. J Agric Food Chem. 2011;59(17):9177-9185. doi:10.1021/jf201950y

32. Sehm J, Lindermayer H, Dummer C, Treutter D, Pfaffl MW. The influence of polyphenol rich apple pomace or red-wine pomace diet on the gut morphology in weaning piglets. J Anim Physiol Anim Nutr (Berl). 2007;91(7-8):289-296.

33. Macagnan FT, Santos LR dos, Roberto BS, de Moura FA, Bizzani M, da Silva LP. Biological properties of apple pomace, orange bagasse and passion fruit peel as alternative sources of dietary fibre. Bioact Carbohydrates Diet Fibre. 2015;6(1):1-6.

34. Ma P, Yao L, Lin X, et al. A mixture of apple pomace and rosemary extract improves fructose consumption-induced insulin resistance in rats: modulation of sarcolemmal CD36 and glucose transporter-4. Am J Transl Res. 2016;8(9):3791-3801.

35. Slavin JL. Dietary fiber and body weight. Nutr . 2005;21(3):411-418.

36. Shalini R, Gupata D, Singh A. Drying kinetics of apple pomace cake. J Food Sci Technol. 2009;46(5):477-479. 
37. Bradbury KE, Appleby PN, Key TJ. Fruit, vegetable, and fiber intake in relation to cancer risk: findings from the European Prospective Investigation into Cancer and Nutrition (EPIC). Am J Clin Nutr. 2014;100(Suppl 1):394S-398S.

38. Park KH, Lee KY, Lee HG. Chemical composition and physicochemical properties of barley dietary fiber by chemical modification. Int J Biol Macromol. 2013;60:360-365.

39. Pereira MA, O'Reilly E, Augustsson K, et al. Dietary fiber and risk of coronary heart disease. Arch Intern Med. 2004;164(4):370-376 .

40. Hwang J, Kim C, Kim C. Extrusion of apple pomace facilitates pectin extraction. J Food Sci. 1998;63(5):841-844.

41. Wang Z, Sun J, Liao X, et al. Mathematical modeling on hot air drying of thin layer apple pomace. Food Res Int. 2007;40(1):39-46.

42. Kumar A, Chauhan GS. Extraction and characterization of pectin from apple pomace and its evaluation as lipase (steapsin) inhibitor. Carbohydr Polym. 2010;82(2):454-459.

43. Mizéhoun-Adissoda C, Houinato D, Houehanou C, et al. Dietary sodium and potassium intakes: Data from urban and rural areas. Nutrition. 2017;33:35-41.

44. Koutsos A, Galvin A, Braune T, et al. Apples and cardiovascular health-is the gut microbiota a core consideration? Ann Nutr Metab. 2015;7(6):3959-3998.

45. USDA. Food Composition Databases Show Foods -- Bananas, raw.

https://ndb.nal.usda.gov/ndb/foods/show/2159?fgcd=\&manu=\&lfacet=\&format=\&count=\&max=5 $0 \&$ offset $=\&$ sort=default\&order $=$ asc\&qlookup=bananas $\& d s=S t a n d a r d+$ Reference \&qt=\&qp $=\& q a=$ $\& q n=\& q=\& i n g=$. Published 2016. Accessed February 9, 2018.

46. USDA. Food Composition Databases Show Foods -- Pears, raw.

https://ndb.nal.usda.gov/ndb/foods/show/2326?manu=\&fgcd=\&ds=Standard Reference.

Published 2016. Accessed February 9, 2018.

47. USDA. Food Composition Databases Show Foods -- Grapes, muscadine, raw. https://ndb.nal.usda.gov/ndb/foods/show/2238?fgcd=\&manu=\&lfacet=\&format=\&count=\&max=5 
$0 \&$ offset $=\&$ sort $=$ default\&order $=$ asc \&qlookup=grapes $\& d s=S t a n d a r d+$ Reference $\& q t=\& q p=\& q a=\&$ $q n=\& q=\& i n g=$. Published 2016. Accessed February 9, 2018.

48. United States Department of Agriculture. Food Composition Databases Show Foods -Oranges, raw, with peel. https://ndb.nal.usda.gov/ndb/foods/show/2288?manu=\&fgcd=. Published 2016. Accessed January 24, 2018.

49. USDA. Food Composition Databases Show Foods -- Blueberries, raw. https://ndb.nal.usda.gov/ndb/foods/show/2166?manu=\&fgcd=\&ds=Standard Reference. Published 2016. Accessed February 9, 2018.

50. Yaegashi Y, Onoda T, Tanno K, Kuribayashi T, Sakata K, Orimo H. Association of hip fracture incidence and intake of calcium, magnesium, vitamin D, and vitamin K. Eur J Epidemiol. 2008;23(3):219-225 .

51. New SA, Millward DJ. Calcium, protein, and fruit and vegetables as dietary determinants of bone health. Am J Clin Nutr. 2003;77(5):1340-1341.

52. Lambert $\mathrm{H}$, Frassetto $\mathrm{L}$, Moore $\mathrm{J}$, et al. The effect of supplementation with alkaline potassium salts on bone metabolism: a meta-analysis. Osteoporos Int. 2015;26(4):1311-1318. 53. Benoist B de, McLean E, Egll I, Cogswell M. Worldwide prevalence of anaemia 19932005: WHO global database on anaemia. Worldw Preval anaemia 1993-2005 WHO Glob database anaemia. 2008.

54. Wessells KR, Brown KH. Estimating the global prevalence of zinc deficiency: results based on zinc availability in national food supplies and the prevalence of stunting. Bhutta ZA, ed. PLoS One. 2012;7(11):e50568.

55. Gazalli $\mathrm{H}$, Malik AH, Sofi AH, et al. Nutritional value and physiological effect of apple pomace. Int J Food Nutr Saf. 2014;5(1):11-15.

56. Gorinstein S, Zachwieja Z, Folta M, et al. Comparative Contents of Dietary Fiber, Total Phenolics, and Minerals in Persimmons and Apples. 2001. 
57. García-Closas R, Berenguer A, Tormo MJ, et al. Dietary sources of vitamin C, vitamin E and specific carotenoids in Spain. Br J Nutr. 2004;91(6):1005-1011.

58. Sun J, Chu Y, Wu X, Liu R. Antioxidant and antiproliferative activities of common Fruits. J Agric Food Chem. 2002;50(25):7449-7454.

59. Pieszka M, Gogol P, Pietras M, Pieszka M. Valuable components of dried pomaces of chokeberry, black currant, strawberry, apple and carrot as a source of natural antioxidants and nutraceuticals in the animal diet. Ann Anim Sci. 2015;15(2):475-491.

60. Lu Y, Foo L. Antioxidant and radical scavenging activities of polyphenols from apple pomace. Food Chem. 2000;68(1):81-85. doi:10.1016/S0308-8146(99)00167-3

61. Schieber A, Hilt P, Streker P, Endreß H-U, Rentschler C, Carle R. A new process for the combined recovery of pectin and phenolic compounds from apple pomace. Innov Food Sci Emerg Technol. 2003;4(1):99-107.

62. Willcox JK, Ash SL, Catigani GL. Antioxidants and prevention of chronic disease. Crit Rev Food Sci Nutr. 2004;44(4):275-295.

63. Hyson DA. A comprehensive review of apples and apple components and their relationship to human health. Adv Nutr. 2011;2(5):408-420. doi:10.3945/an.111.000513 64. Chinnici F, Bedini A, Gaiani A, Riponi C. Radical scavenging activities of peels and pulps from cv. Golden Delicious apples as related to their phenolic composition. J Agric Food Chem. 2004;52(15):4684-4689.

65. Taso R, Yang R, Young J, Zhu H. Polyphenolic profiles in eight apple cultivars using high-performance liquid chromatography (HPLC). J Agric Food Chem. 2003;51(21):6347-6353.

66. Boyer J, Liu RH. Apple phytochemicals and their health benefits. Nutr J. 2004;3(1):5.

67. Vanzani P, Rossetto M, Rigo A, et al. Major phytochemicals in apple cultivars: contribution to peroxyl radical trapping efficiency. J Agric Food Chem. 2005;53(9):3377-3382 .

68. Brecht JK. Physiology of Lightly Processed Fruits and Vegetables. HortScience. 1995;30(1):18-22. 
69. Krawitzky M, Arias E, Peiro JM, Negueruela AI, Val J, Oria R. Determination of color, antioxidant activity, and phenolic profile of different fruit tissue of Spanish "Verde Doncella" apple cultivar. Int J Food Prop. 2014;17(10):2298-2311.

70. Saucier CT, Waterhouse AL. Synergetic Activity of Catechin and Other Antioxidants. J Agric Food Chem. 1999;47(11):4491-4494.

71. Diñeiro García Y, Valles BS, Picinelli Lobo A. Phenolic and antioxidant composition of by-products from the cider industry: Apple pomace. Food Chem. 2009;117(4):731-738.

72. Belding R, Blankenship S, Young E, Leidy RB. Composition and variability of epicuticular waxes in apple cultivars. J Am Soc Hortic Sci. 1998;123(3):348-356.

73. Eigenbrode SD, Espelie KE. Effects of plant epicuticular lipids on insect herbivores. Annu Rev Entomol. 1995;40(1):171-194.

74. Whitaker B. Phenolic fatty-acid esters from the peel of Gala apples and their possible role in resistance to superficial scald. Postharvest Biol Technol. 1998;13(2):1-10.

75. Frighetto RTS, Welendorf RM, Nigro EN, Frighetto N, Siani AC. Isolation of ursolic acid from apple peels by high speed counter-current chromatography. Food Chem. 2008;106(2):767771.

76. Balanehru S, Nagarajan B. Intervention of adriamycin induced free radical damage. Biochem Int. 1992;28(4):735-744.

77. Gupta MB, Bhalla TN, Gupta GP, Mitra CR, Bhargava KP. Anti-inflammatory activity of natural products triterpenoids. Eur J Pharmacol. 1969;6(1):67-70.

78. Singh GB, Singh S, Bani S, Gupta BD, Banerjee SK. Anti-inflammatory activity of oleanolic acid in rats and mice. J Pharm Pharmacol. 1992;44(5):456-458.

79. Ohigashi H, Takamura H, Koshimizu K, Tokuda H, Ito Y. Search for possible antitumor promoters by inhibition of 12-O-tetradecanoylphorbol-13-acetate-induced Epstein-Barr virus activation; Ursolic acid and oleanolic acid from an anti-inflammatory Chinese medicinal plant, Glechoma hederaceae L. Cancer Lett. 1986;30(2):143-151. 
80. Niikawa M, Hayashi $\mathrm{H}$, Sato $\mathrm{T}$, Nagase $\mathrm{H}$, Kito $\mathrm{H}$. Isolation of substances from glossy privet (Ligustrum lucidum Ait.) inhibiting the mutagenicity of benzo[a]pyrene in bacteria. Mutat Res Toxicol. 1993;319(1):1-9.

81. Guevara AP, Amor E, Russell G. Antimutagens from Plumeria acuminata ait. Mutat Res Mutagen Relat Subj. 1996;361(2-3):67-72.

82. Ikeda Y, Murakami A, Ohigashi H. Ursolic acid: An anti- and pro-inflammatory triterpenoid. Mol Nutr Food Res. 2008;52(1):26-42.

83. Babalola IT, Adelakun EA, Shode FO. Isolation of ursolic acid (3ß-hydroxyurs-12-en-28oic acid) from the leaves of Eucalyptus grandis. Phyther Res. 2013;5(5):33-37.

84. Farneti B, Masuero D, Costa F, et al. Is there room for improving the nutraceutical composition of apple? J Agric Food Chem. 2015;63(10):2750-2759.

85. Grigoras C, Destandau E, Fougere L, Elfakir C. Evaluation of apple pomace extracts as a source of bioactive compounds. Ind Crops Prod. 2013;49:794-804. doi:10.1016/J.INDCROP.2013.06.026

86. Innocente A, Silva G, Cruz L, et al. Synthesis and antiplasmodial activity of betulinic acid and ursolic acid analogues. Molecules. 2012;17(12):12003-12014.

87. Caliceti C, Calabria D, Roda A, Cicero A. Fructose intake, aerum uric acid, and cardiometabolic disorders: a critical review. Nutrients. 2017;9(4):395.

88. Krishnan E, Kwoh C, Schumacher H, Kuller LH. Hyperuricemia and incidence of hypertension among men without metabolic syndrome. Hypertension. 2007;49(2):298-303 .

89. Nakagawa T, Hu H, Zharikov S, et al. A causal role for uric acid in fructose-induced metabolic syndrome. Am J Physiol Physiol. 2006;290(3):F625-F631. doi:10.1152/ajprenal.00140.2005

90. Edwards N. The role of hyperuricemia and gout in kidney and cardiovascular disease. Cleve Clin J Med. 2008;75:S13-16. 
91. Dehghan A, Van Hoek M, Siijbrands E, Hoffman A, Witteman J. High serum uric acid as a novel risk factor for type 2 diabetes. Diabetes Care. 2008;31(2):361-362.

92. Schretlen D, Inscore A, Jinnah H, Rao V, Gordon B, Pearlson G. Serum uric acid and cognitive function in community-dwelling older adults. Neuropsychology. 2007;12(1):136.

93. Kim DH, Jin YH. Intestinal bacterial $\beta$-glucuronidase activity of patients with colon cancer. Arch Pharm Res. 2001;24(6):564-567.

94. Heinritz S, Mosenthin R, Weiss E. Use of pigs as a potential model for research into dietary modulation of the human gut microbiota. Nutr Res Rev. 2013;26(2):191-209.

95. Kim Y, Keogh J, Clifton P. Polyphenols and glycemic control. Nutrients. 2016;8(1):17.

96. Andollahzadeh F, Pirmohammadi R, Fatehi F, Bernousi I. Effect of feeding ensiled tomato and apple pomace on performance of Holstein dairy cows. Slovak J Anim Sci. 2010;43(1):31-35.

97. Tiwari S, Narang M, Dubey M. Effect of feeding apple pomace on milk yield and milk composition in crossbred (Red Sindhi x Jersey) cow. Livest Res Rural Dev. 2008;20(4):293-297. 98. Narang MP, Lal R. Evaluation of some agro-industrial wastes in the feed of Jersey calves. Agric Wastes. 1985;13(1):15-21.

99. Ahn J, Jo I, Lee J. The use of apple pomace in rice straw based diets of Korean native goats (Capra hircus). Asian-Austrian J Anim Sci. 2002;15(11):1599-1605.

100. Bolarinwa IF, Orfila C, Morgan MRA. Amygdalin content of seeds, kernels and food products commercially-available in the UK. Food Chem. 2014;152:133-139.

101. Montagnac JA, Davis CR, Tanumihardjo SA. Processing techniques to reduce toxicity and antinutrients of cassava for use as a staple food. Compr Rev Food Sci Food Saf. 2009;8(1):17-27.

102. Haque R, Bradbury $\mathrm{H}$. Total cyanide determination of plants and foods using the picrate and acid hydrolysis methods. Food Chem. 2002;77(1):107-114. 
103. Opyd P, Jurgoński A, Juśkiewicz J, Milala J, Zduńczyk Z, Król B. Nutritional and HealthRelated Effects of a Diet Containing Apple Seed Meal in Rats: The Case of Amygdalin. Nutrients. 2017;9(10):1091.

104. National Institutes of Health. TOXNET: Toxicology Data Network, hydrogen cyanide. National Institutes of Health Toxicology Data Network. https://toxnet.nlm.nih.gov/cgibin/sis/search/a?dbs+hsdb:@term+@DOCNO+165. Published 2017. Accessed January 24, 2018.

105. Zhang J, Wang $\mathrm{Y}$, Xiang $\mathrm{H}$, et al. Oxidative stress: role in acetamiprid-induced impairment of the male mice reproductive system. Agric Sci China. 2011;10(5):786-796. 106. Chen M, Tao L, McLean J, Lu C. Quantitative analysis of neonicotinoid insecticide residues in foods: implication for dietary exposures. J Agric Food Chem. 2014;62(26):60826090.

107. EPA. Name of Chemical: Acetamiprid. 2002.

https://www3.epa.gov/pesticides/chem_search/reg_actions/registration/fs_PC-099050_15-Mar02.pdf. Accessed January 17, 2018.

108. Lozowicka B. Health risk for children and adults consuming apples with pesticide residue. Sci Total Environ. 2015;502:184-198.

109. Liu S, Che Z, Chen G. Multiple-fungicide resistance to carbendazim, diethofencarb, procymidone, and pyrimethanil in field isolates of Botrytis cinerea from tomato in Henan Province, China. Crop Prot. 2016;84:56-61.

110. EPA. Pesticide fact sheet: Cyprodinil. 1998.

https://www3.epa.gov/pesticides/chem_search/reg_actions/registration/fs_PC-288202_06-Apr98.pdf. Accessed January 17, 2018.

111. Maiti B, Desai S, Krishnamoorthy T. Determination of naphthaleneacetic acid residue in apples by high-performance liquid chromatography. Analyst. 1988;113(4):667-668. 
112. EPA. Reregistration Eligibility Decision (RED): Napthalenacetic Acid. 2007.

https://archive.epa.gov/pesticides/reregistration/web/pdf/naa_amendment.pdf. Accessed January 17, 2018.

113. Liu S, Huang X, He H, Jin Q, Zhu G. Evaluation of selected plant growth regulators and fungicide residues in fruits for dietary risk assessment. Hum Ecol Risk Assess An Int J. 2016;22(6):1386-1395.

114. EPA. R.E.D. Facts: Diphenylamine. 1998.

https://archive.epa.gov/pesticides/reregistration/web/pdf/221 0fact.pdf. Accessed January 17, 2018.

115. USDA. Pesticide Data Program | Agricultural Marketing Service.

https://www.ams.usda.gov/datasets/pdp. Published 2015. Accessed January 17, 2018.

116. Shalini R, Gupta DK. Utilization of pomace from apple processing industries: a review. J Food Sci Technol. 2010;47(4):365-371. doi:10.1007/s13197-010-0061-x

117. Bralt A, de Oliveira D. Seed number and asymmetry index of McIntosh apples. HortScience. 1995;30(1):44-46.

118. Yokel J, Delistraty DA. Arsenic, lead, and other trace elements in soils contaminated with pesticide residues at the Hanford site (USA). Environ Toxicol. 2003;18(2):104-114.

119. Wilson D, Hooper C, Shi X. Arsenic and lead in juice: apple, citrus, and apple-base. J Environ Health. 2012;75(5):14-21.

120. Punshon T, Jackson BP, Meharg AA, Warczack T, Scheckel K, Guerinot M Lou. Understanding arsenic dynamics in agronomic systems to predict and prevent uptake by crop plants. Sci Total Environ. 2017;581:209-220.

121. Arslan B, Djamgoz MBA, Akün E. Arsenic: a review on exposure pathways, accumulation, mobility and transmission into the human food chain. Environ Contam Toxicol. 2016;243:27-51 . 
122. Keikothaile BM, Spanoghe $\mathrm{P}$, Steurbaut W. Effects of food processing on pesticide residues in fruits and vegetables: A meta-analysis approach. Food Chem Toxicol. 2010;48(1):16.

123. Kaushik G, Satya S, Naik SN. Food processing a tool to pesticide residue dissipation - A review. Food Res Int. 2009;42(1):26-40.

124. Soliman K. Changes in concentration of pesticide residues in potatoes during washing and home preparation. Food Chem Toxicol. 2001;39(8):887-891 .

125. Yang T, Doherty J, Zhao B, Kinchla AJ, Clark JM, He L. Effectiveness of commercial and homemade washing agents in removing pesticide residues on and in apples. J Agric Food Chem. 2017;65(44):9744-9752.

126. Bonmatin J-M, Giorio C, Girolami V, et al. Environmental fate and exposure; neonicotinoids and fipronil. Environ Sci Pollut Res. 2015;22(1):35-67. 127. EPA. Reference Dose (RfD): description and use in health risk assessments. https://www.epa.gov/iris/reference-dose-rfd-description-and-use-health-risk-assessments. Accessed January 17, 2018.

128. EPA. Choosing a percentile of acute dietary exposure as a threshold of regulatory concern. https://www.epa.gov/sites/production/files/2015-07/documents/trac2b054_0.pdf. Published 2000. Accessed January 17, 2018.

129. Lee H, Kim E, Moon JK, et al. Establishment of analytical method for cyazofamid residue in apple, mandarin, korean cabbage, green pepper, potato and soybean. J Korean Soc Appl Biol Chem. 2012;55(2):241-247.

130. Bempah CK, Asomaning J, Boateng J. Market basket survey for some pesticide residues in fruits and vegetables from Ghana. J Microbiol Biotechnol Food Sci. 2012;2(3):850871. 
131. EPA. US EPA - pesticides - fact sheet for Thiophanate-methyl.

https://www3.epa.gov/pesticides/chem_search/reg_actions/reregistration/fs_PC-102001_1-Nov04.pdf. Accessed January 17, 2018.

132. Masia A, Ventura M, Gemma H, Sansavini S. Effect of some plant growth regulator treatments on apple fruit ripening. Plant Growth Regul. 1998;25(2):127-134.

133. Min B, Bae IY, Lee HG, Yoo S-H, Lee S. Utilization of pectin-enriched materials from apple pomace as a fat replacer in a model food system. Bioresour Technol. 2010;101(14):54145418.

134. Sudha ML, Baskaran V, Leelavathi K. Apple pomace as a source of dietary fiber and polyphenols and its effect on the rheological characteristics and cake making. Food Chem. 2007;104(2):686-692. doi:10.1016/J.FOODCHEM.2006.12.016

135. Carson K, Collins J, Penfield M. Unrefined, Dried Apple Pomace as a Potential Food Ingredient. J Food Sci. 1994;59(6):1213-1215.

136. Issar K, Sharma PC, Gupta A. Utilization of Apple Pomace in the Preparation of FiberEnriched Acidophilus Yoghurt. J Food Process Preserv. 2017;41(4):e13098.

137. Wang H, Thomas R. Direct Use of Apple Pomace in Bakery Products. J Food Sci. $1989 ; 54(3): 618-620$.

138. Bhushan S, Kalia K, Sharma M, Singh B, Ahuja PS. Processing of apple pomace for bioactive molecules. Crit Rev Biotechnol. 2008;28(4):285-296.

doi:10.1080/07388550802368895 
Table 1. Comparison of the nutrient composition of whole apples versus apple pomace

\begin{tabular}{lll}
\hline Constituents (Fresh weight) & Whole Apple & Apple Pomace \\
\hline Macronutrients (\%) & & \\
Fat & $0.16-0.18$ & $1.1-3.6$ \\
Protein & $0.24-0.28$ & $2.7-5.3$ \\
Total Carbohydrate & 13.81 & $44.5-57.4$ \\
Simple Carbohydrates (\%) & & \\
Fructose & $5.8-6.0$ & 44.7 \\
Glucose & $2.4-2.5$ & $18.1-18.3$ \\
Complex Carbohydrates (\%) & & \\
Total Fiber & $2.1-2.6$ & $4.4-47.3$ \\
Insoluble Fiber & 1.54 & $33.8-60.0$ \\
Soluble Fiber & 0.67 & $13.5-14.6$ \\
Pectin & $0.71-0.93$ & $3.2-13.3$ \\
Major Minerals (mg/100g) & & \\
Sodium & $0.9-1.1$ & 185.3 \\
Potassium & $104.8-109.2$ & $398.4-880.2$ \\
Calcium & $5.7-6.3$ & $55.6-92.7$ \\
Phosphorus & $10.7-11.3$ & $64.9-70.4$ \\
Magnesium & $4.9-5.1$ & $18.5-333.5$ \\
Trace Elements (mg/100g) & & \\
Iron & $0.11-0.13$ & $2.9-3.5$ \\
Zinc & $0.0036-0.0044$ & 1.4 \\
Copper & $0.026-0.028$ & 0.1 \\
Manganese & $0.033-0.037$ & $0.4-0.8$ \\
\hline Val
\end{tabular}

Values are the ranges reported for $n=6-38$ samples. A single value indicates an $n=1$ sample. ${ }^{1}$ Values for whole apples are based on the USDA database.[1] ${ }^{2}$ Values for apple pomace are based on Bhushan, et al.[2] and Queji, et al.[3] 
Table 2. Mineral values in commonly consumed fruits in North America.

\begin{tabular}{|c|c|c|c|c|c|c|c|}
\hline $\begin{array}{l}\text { Dietary } \\
\text { Minerals } \\
\text { mg/100 g Fresh } \\
\text { Weight }\end{array}$ & Apples $^{1}$ & Bananas $^{1}$ & Pears $^{1}$ & Grapes $^{1}$ & Oranges $^{1}$ & Blueberries $^{1}$ & Apple Pomace ${ }^{2}$ \\
\hline Sodium & $1 \pm 0.07$ & $1 \pm 0.40$ & $1 \pm 0.20$ & $0.4 \pm 0.44$ & $0 \pm 0.03$ & $0.16 \pm 0.35$ & $185.3 \pm 0.00$ \\
\hline Potassium & $107 \pm 2.21$ & $358 \pm 1.91$ & $116 \pm 3.61$ & $191 \pm 27.52$ & $181 \pm 1.40$ & $77 \pm 5.45$ & $639.3 \pm 240.9$ \\
\hline Calcium & $6 \pm 0.34$ & $5 \pm 0.05$ & $9 \pm 0.41$ & $14 \pm 1.72$ & $43 \pm 2.24$ & $6 \pm 0.79$ & $74.1 \pm 18.5$ \\
\hline Phosphorus & $11 \pm 0.34$ & $22 \pm 0.17$ & $12 \pm 0.23$ & $10 \pm 0.61$ & $14 \pm 0.44$ & $12 \pm 0.51$ & $67.6 \pm 2.8$ \\
\hline Magnesium & $5 \pm 0.07$ & $27 \pm 0.48$ & $7 \pm 0.07$ & $5 \pm 0.18$ & $10 \pm 0.17$ & $6 \pm 0.20$ & $176.0 \pm 157.5$ \\
\hline Iron & $0.12 \pm 0.01$ & $0.26 \pm 0.001$ & $0.18 \pm 0.03$ & $0.29 \pm 0.06$ & $0.1 \pm 0.04$ & $0.28 \pm 0.11$ & $3.2 \pm 0.3$ \\
\hline Zinc & $0.04 \pm 0.004$ & $0.15 \pm 0.001$ & $0.10 \pm 0.004$ & $0.04 \pm 0.01$ & $0.07 \pm 0.00$ & $0.16 \pm 0.02$ & $1.4 \pm 0.00$ \\
\hline
\end{tabular}

Values report as mean \pm SE for $n=1-212$ samples.

${ }^{1}$ Values for apples, bananas, pears, grapes, oranges, and blueberries are from USDA.[1,4-8] ${ }^{2}$ Apple pomace values are from Bhushan, et al.[2] 
Table 3. Studies of apple pomace constituents and whole apple pomace effects on health.

\section{Reference \\ Apple Pomace \\ Constituents}

Chen, et al. (2017) [23]

[24]

Sembries, et al. (2006) [25]

Sembries, et al. (2004)

\section{Apple pomace \\ Human Studies}

Dietary study characteristics

Results
Male Kunming mice age 45d

$\mathrm{n}=18$ animals/group

$45 \%$ HFD with 200,400 , or

pomace polysaccharide

extract for 30 days
$800 \mathrm{mg} / \mathrm{kg}$ bwt/d apple

\section{$200 \mathrm{mg} / \mathrm{kg} \mathrm{bwt} / \mathrm{d}$}

$\downarrow$ serum cholesterol

$\downarrow$ serum insulin

$\downarrow$ serum adiponectin

$\downarrow$ serum leptin

\section{0 and $400 \mathrm{mg} / \mathrm{kg} \mathrm{bwt/d}$}

\section{$\downarrow$ serum LDL-C}

\section{All doses}

$\downarrow$ fasting serum insulin

$\downarrow$ serum hexokinase and glucagon

$\downarrow$ liver lipid

$\uparrow$ serum antioxidant capacity dose-dependent

Male Wistar rats age 8 weeks $\mathrm{n}=12$ animals/group

Standard rodent diet with $5 \%$ apple pomace fiber-rich colloid for 6 weeks

Male Wistar rats age 6 weeks $\mathrm{n}=10$ animals/group

Standard rodent diet $+5 \%$ apple pomace fiber-rich colloids for 6 weeks

$\uparrow$ cecum SCFA, acetate, and proprionate

$\uparrow$ fecal bile acid excretion

$\downarrow$ body weight $\downarrow$ serum $\mathrm{CHL}$

$\downarrow$ serum LDL-C

$\uparrow$ serum HDL-C

$\uparrow$ fecal bile acid and neutral sterol excretion
Men and women age 18-69 years $(n=34)$

Polyphenol and pectin

restricted diet $+22 \mathrm{mg} /$ day
NS lipid profile and bile acid $\downarrow$ fecal lithocholic acid excretion 
apple pomace for $5 \times 4$

weeks crossover

Rago, et al. (2015) [27]

Men and women age 18-69

years $(n=34)$

Polyphenol and pectin

restricted diet $+22 \mathrm{mg} / \mathrm{d}$

apple pomace for $5 \times 4$

weeks crossover

\section{Preclinical Studies}

Cho, et al. (2013) [28]

Bobek, et al. (1998) [29] 30]

\section{Male Sprague Dawley rats age 3 weeks $(n=8$ \\ animals/group) \\ $15 \%$ HFD $+10 \%$ apple \\ pomace for 9 weeks}

Male Wistar rats age 3 weeks $\mathrm{n}=20$ animals/group

$0.3 \% \mathrm{CHL}+5 \%$ apple

pomace for 10 weeks

Male Wistar rats age $35 \mathrm{~d}$

$\mathrm{n}=8$ animals/group

Standard diet $+15 \%$

unprocessed or $14 \%$

processed apple pomace for

$28 \mathrm{~d}$
Plasma measurements:

$\downarrow$ aromatic amino acids

$\downarrow$ medium- and short-chain acylcarnitines

$\downarrow$ primary bile acids

$\downarrow$ deydropiandrosterone sulphate, and lysophospholipids

$\uparrow$ uric acid

\author{
$\downarrow$ body weight and fat \\ $\downarrow$ serum LDL-C \\ $\uparrow$ serum HDL-C \\ $\downarrow$ liver $\mathrm{CHL}$ and TG \\ $\uparrow$ fecal $\mathrm{CHL}$ and TG
}

$\downarrow$ liver $\mathrm{CHL}$

$\downarrow$ liver HMG-CoA reductase

$\downarrow$ plasma conjugated dienes

$\uparrow$ fractional catabolic rate of plasma $\mathrm{CHL}$

$\downarrow$ erythrocyte SOD, catalase, GPx activity

\section{Unprocessed apple pomace}

$\uparrow$ erythrocyte SOD

$\uparrow$ serum antioxidant capacity of fat and water-soluble substances

$\downarrow$ urine nitrogen

$\downarrow$ digesta $\beta$-glucosidase

$\downarrow$ serum glucose

$\downarrow$ liver TBARS

\section{Processed apple pomace}

$\uparrow$ antioxidant capacity of water-soluble substances

$\downarrow$ digesta $\beta$-glucosidase and $\beta$-glucuronidase 
$\downarrow$ colon digesta $\mathrm{pH}$

$\downarrow$ fecal $\mathrm{pH}$

\section{All apple pomace groups}

$\downarrow$ fecal nitrogen

$\downarrow$ nitrogen digestibility

$\uparrow$ cecum tissue weight, digesta, dry matter, $\mathrm{pH}$, ammonia

$\uparrow$ cecal digesta SCFA

Kosmala, et al. (2011) [31] Male Wistar rats age 4 weeks $\mathrm{n}=8$ animals/group

Standard diet $+0.23 \%(\mathrm{w} / \mathrm{w})$ whole apple pomace, $0.10 \%$ (w/w) flavonoid-reduced apple pomace, or $0.01 \%$ $(\mathrm{w} / \mathrm{w})$ flavonoid-deprived apple pomace for 4 weeks
Sehm, et al. (2007) [32] [33]
Macagnan, et al. (2015)

\section{Whole apple pomace}

$\uparrow$ cecum tissue weight

$\downarrow \beta$-glucuronidase

$\downarrow$ serum glucose

Flavonoid-reduced apple pomace

$\uparrow$ cecal microbiota glycolytic activity

Modified cecal SCFA:BCFA ratio

Flavonoid-deprived

$\downarrow$ cecal ammonia

$\downarrow$ colonic $\mathrm{pH}$

\section{$\downarrow$ serum glucose}

\section{All apple pomace groups}

$\uparrow$ intestinal fermentation

$\uparrow$ fecal SCFA

$\downarrow$ cecum $\mathrm{pH}$

Male Pietráin $\times$ (Deutsche

NS feed/energy intake, weight gain

$\uparrow$ jejunum and ileum villi breadth

Landrasse $\times$ Deutsches

Edelschwein) piglets age 24d

$\downarrow$ gut-associated lymphoid tissue

$\mathrm{n}=39$ animals/group

$3.5 \%$ apple pomace for 6 weeks

Male Wistar rats age 3 weeks
$\mathrm{n}=8$ animals/group

Standard diet $+68.8 \mathrm{~g} / \mathrm{kg}$ of $\quad \downarrow$ dry fecal production 
Male Sprague-Dawley rats age 7 weeks

$\mathrm{n}=6-9$ animals/group

$10 \%$ fructose in water + standard diet with $100 \mathrm{mg} / \mathrm{kg}$ or $500 \mathrm{mg} / \mathrm{kg}$ apple pomace + rosemary extract for 5 weeks

Abbreviations and symbols: $\downarrow$, decrease; $\uparrow$, increase; Adipo-IR, adipose tissue insulin resistance; AUC, area under the curve; BCFA, branched-chain fatty acid; CHL, cholesterol; HDL-C, high density lipoprotein-cholesterol; GPx, glutathione peroxidase; HFD, high fat diet; HOMA-IR, homeostatic model assessment for insulin resistance; LDH, lactate dehydrogenase; LDL-C, low density lipoproteincholesterol; NS non-significant; SCFA, short-chain fatty acids; SOD, super oxide dismutase; TBARS, thiobarbituric acid reactive substances; TG, triacylglycerol.

\section{$500 \mathrm{mg} / \mathrm{kg}$ apple pomace}

$\downarrow$ fasting plasma glucose

$\downarrow$ gastrocnemius sarcolemmal CD36 and GLUT-4 stain

\section{intensity}

Both apple pomace groups

$\downarrow$ plasma insulin, HOMA-IR, and Adipo-IR 
Table 4. Studies on safety of apple and apple constituents and pesticide safety information.

\section{Reference \\ Amygdalin}

Bolarinwa, et al. (2014) [100]

Safety study characteristics

Seeds from 15 apple varieties

Opyd, et al. (2017) [103]

NIH (2017) [104]

\section{Pesticides}

\section{Neonicotinoids}

Zhang, et al. (2011) [105]

Results

$\uparrow$ amygdalin (2.80-3.91 mg/g) in Golden Delicious, Royal Gala, and Red Delicious $\downarrow$ amygdalin (0.95-1.19 mg/g) Braeburn and Egremont Russet

Male Wistar rats age 8 weeks $\mathrm{n}=10$ animals/group

High-saturated fat (7\% lard, $1 \%$ $\mathrm{CHL}$ ) and high-fructose (68.75\%) diet with $0.24 \%$ amygdalin or $18.4 \%$ apple seeds $(0.24 \%$ amygdalin)

\section{Toxicology Database}

Male Kungming mice age 8 weeks

$\mathrm{n}=10$ animals/group

Oral ingestion of $30 \mathrm{mg} / \mathrm{kg} \mathrm{bwt} / \mathrm{d}$ acetamiprid for $35 \mathrm{~d}$
Amygdalin dose equivalent to $160 \mathrm{mg} / \mathrm{kg}$ Apple Seeds

$\downarrow$ food consumption

$\downarrow$ body weight

$\downarrow$ protein digestibility and nitrogen retention

$\uparrow$ cecum and digesta mass

$\uparrow$ cecum microbial enzyme activity

$\uparrow$ digeta SCFA

$\uparrow$ serum HDL-C

$\uparrow$ serum antioxidant capacity of water-

soluble substances

$\downarrow$ liver TBARS

Hydrogen cyanide LD=50-300 mg

$=$ need to consume $\sim 800 \mathrm{~g}$ apple pomace

$\sim 30 \mathrm{mg} / \mathrm{kg}=$ toxicity in humans

$\downarrow$ testis, epididymis, seminal vesicle, prostate weight

$\uparrow$ Leydig cell damage

$\uparrow$ testicular p38 MAPK

$\downarrow$ testicular catalase, GPX, and SOD

$\downarrow$ sperm count, viability, and motility, rate of

intact acrosomes

$\downarrow$ serum testosterone 
Chen, et al. (2014) [106]

$$
\text { EPA (2002) [107] }
$$

\section{Fungicides}

Lozowicka (2015) [108]

Liu, et al. (2016) [109]

\section{Plant Growth Regulators}

Maiti, et al. (1988) [111]

Liu, et al. (2016) [113]

Lozowicka (2015) [108]
Acetamiprid Fact Sheet

Apples $\mathrm{n}=696$

Apples $n=24$

Apples $\mathrm{n}=7$

Whole apples

Apples without skin

Apple skin

Apples $n=24$

Apples $\mathrm{n}=696$ $\uparrow$ acetamiprid Granny Smith $(0.407 \mathrm{mg} / \mathrm{kg})$ and Honey Crisp $(0.1007 \mathrm{mg} / \mathrm{kg})$

Acute $\mathrm{RfD}=0.10 \mathrm{mg} / \mathrm{kg} / \mathrm{d}$

Chronic RfD $=0.07 \mathrm{mg} / \mathrm{kg} / \mathrm{d}$

$\% \mathrm{PAD}=10-40$

Dithocarbamate residue found most frequently on apples

Risk at $100 \%$

Hazard index and acute reference dose 0$4.65 \%$

Thiophanate $(2 \mathrm{mg} / \mathrm{kg})$ and found within allowed residue levels

Thiophanate and carbendazim low \% PAD $\uparrow p y r i m e t h a n i l$ compared to other fruits EPA (1998)[29] states cyprodinil (similar to pyrimethanil) \% PAD $=5.8$

$\uparrow$ Naphthaleneacetic acid residue on apple skin $(0.433 \mathrm{mg} / \mathrm{kg})$

Naphthaleneacetic acid in whole apples and without skin $(0.042-0.285 \mathrm{mg} / \mathrm{kg})$

EPA (2007)[31] states naphthaleneacetic acid poses no acute or chronic health risks

No detectable PGR residue

Diphenylamine found on $14.6 \%$ of apples EPA(1998)[33] states low risk for toxicity Tolerance level of 10 ppm (apples) and 30 ppm (apple pomace) 

All below EPA tolerance level

Abbreviations and symbols: $\downarrow$, decrease; $\uparrow$, increase ; EPA, Environmental Protection Agency; NIH, National Institutes of Health; NS, non-significant; PGR, plant growth regulator; ppm, parts per million; RfD, reference dose; \% PAD, \% population adjusted dose; TBARS, thiobarbituric acid reactive substances; USDA, United States Department of Agriculture. 


\subsection{Diet-induced non-alcoholic fatty liver disease: a brief review}

Non-alcoholic fatty liver disease (NAFLD) is the most prevalent liver disease worldwide with prevalence also rising in children [1,2]. NAFLD is defined as hepatic steatosis not caused by alcohol consumption and is often linked to overconsumption of saturated fat and simple carbohydrates as well as obesity [3-5]. NAFLD can progress to the more severe non-alcoholic steatohepatitis (NASH), which includes inflammation in addition to steatosis [6]. As the disease progresses from NAFLD to NASH, mortality and systemic complications increase $[7,8]$. Approximately $25 \%$ of the world's population is reported to have NAFLD, with $25 \%$ of these individuals having the more severe NASH [9]. However, prevalence may be higher since liver biopsy is most accurate diagnostic for the disease is costly and invasive [10]. As the obesity epidemic continues, cases of NAFLD and NASH, with current predications forecasting a potential liver disease crisis $[9,11]$.

\subsubsection{Etiology}

NAFLD has been suggested to be the hepatic manifestation of metabolic syndrome [12]. Poor diet and lack of physical exercise are factors in NAFLD development [1]. NAFLD prevalence is particularly high in Western countries, due to "Western diets" defined as diets high in saturated fat and simple carbohydrates [13,14]. A multiple-hit model has been proposed to explain NAFLD progression, with the first hit being accumulation of fat in the liver and the second hit being the onset of inflammation [4].

Accumulation of hepatic fat results from dysregulation of hepatic lipid metabolism. Overconsumption of saturated fat and simple carbohydrates results in increased i lipogenesis (DNL) through the stimulation of genes involved in the DNL cascade $[15,16]$. Transcription factors sterol regulatory binding protein-1c (SREBP-1c) and carbohydrate element response binding protein (ChREBP) stimulate fatty acid synthase (FAS) to catalyze the synthesis of saturated fatty acids (SFAs) [15]. These SFAs can then be further desaturated to monounsaturated fatty acids (MUFAs) by stearoyl-CoA desaturase (SCD-1), which are more 
readily used for triglyceride synthesis [15,17]. Esterification is catalyzed by diacylglycerol O-acyl transferase-2 (DGAT2), resulting in the accumulation of hepatic triglycerides [18]. Triglycerides can accumulate in the liver or enter circulation via activation by microsomal triglyceride transport protein (MTTP), which stimulates very-low density lipoprotein (VLDL) production and subsequent circulation (Figure 1) [19].

Rise in circulating VLDL impairs bile acid homeostasis [20]. Typically, bile acids assist in digestion and absorption of fats and fat-soluble vitamins, cholesterol homeostasis, and eliminates waste from the body [20, 21]. Bile acids also act as metabolic signaling molecules, with normal reabsorption critical for triglyceride and glucose metabolism homeostasis [21]. Individuals with NAFLD have elevated bile acid production which contributes to elevated levels of circulating fatty acids and glucose that eventually leads to increased fat deposition and a proinflammatory state [22-24]. Additionally, bile acid dysregulation along with increased DNL can increase circulating free fatty acids [25]. Increases in MUFAs have been shown to increased triglyceride production and storage [26]. While increases in SFAs has been linked to increased inflammation, a second hit in the NAFLD cascade [27].

Increases in SFAs has been shown to stimulate inflammatory gene expression, through activation of transcription factor nuclear factor kappa-light enhancer of activated B cells (NFkB) [28]. NFKB activates downstream inflammatory cytokines, such as IL- 6 and TNF- $\alpha$, resulting in increased hepatic inflammation [4,29]. This inflammatory cascade is not limited to the liver, as adipose tissue is also implicated in the inflammatory process. Alterations in fatty acid and glucose metabolism result in in adipose tissue lipogenesis leading to alterations in adipose tissue fatty acid profiles and inflammation status, further exacerbating increased hepatic DNL [30-32]. Inflammatory cytokines, upregulated by SFA, are transported to the liver, further promoting inflammation [4,30]. Inflammation coupled with increased reactive oxygen species (ROS) generation from free fatty acid metabolism promotes NAFLD progression to the more severe NASH (Figure 2) [6]. 
Further complicating this progression, polyunsaturated fatty acids (PUFAs) can also exacerbate inflammation and lipid peroxidation in NASH [14,33]. Overconsumption of the $n-6$ PUFA, linoleic acid can result in increased inflammation and exacerbate liver disease [34]. Current recommendation are for the ratio of linoleic acid to n-3 PUFA $\alpha$-linolenic acid to be approximately 1:1. The Western diet has a linoleic:a-linolenic acid ratio of 15:1 [14,34]. This essential fatty acid imbalance increases metabolism of linoleic acid to arachidonic acid [35]. Arachidonic acid can be further metabolized by cyclooxygenases to bioactive compounds, 2 series prostaglandins and thromboxanes or to 4 series leukotrienes by lipooxygenases [36,37]. Further, the ratio of $n-6: n-3$ has been shown to be the most important determinant of cell membrane composition, with an increase in $n-6$ consumption leading to a proinflammatory state [38].

Increases in inflammatory cytokines from the liver and adipose tissue can be circulated to the kidney, resulting in renal inflammation and vascular dysfunction $[4,39]$. Increased uric acid production, due to overconsumption of sucrose and fructose, further exacerbates inflammation and increases risk of developing kidney stones, gout, and other renal diseases [40-42] [43,44]. Additionally, recent research suggests as NAFLD progresses it can result in detriments to bone health. Individuals with NAFLD have been shown to have decreased bone mineral density, and to increase the risk of osteoporosis [45-47]. Chronic inflammation increases in inflammatory cytokines, and decreased antioxidant defense have been suggested as a link between NAFLD and decreased bone mineral density [47].

NAFLD is reversible with lifestyle modifications. However, as NAFLD progresses from simple steatosis to NASH reversibility decreases [6]. Continual consumption of a diet high in saturated fat and simple carbohydrates without intervention can lead to the onset of $\mathrm{NASH}$, and eventual cirrhosis [6,48]. Cirrhosis of the liver is defined as hepatic steatosis, inflammation, and fibrosis [49]. Cirrhosis is advanced stage liver disease that is irreversible and significantly increases risk of mortality $[49,50]$. Currently, no Food and Drug Administration approved drugs 
exist for NAFLD treatment, medications are recommended to slow progression of cirrhosis, along with lifestyle modifications [51]. Cirrhosis of the liver also further increases the risk for chronic kidney disease, osteoporosis, and development of hepatocellular carcinoma $[5,6,52,53]$. Drug and lifestyle interventions slows the progression of cirrhosis, however liver transplant the only effective remedy [49]. NAFLD prevalence is increasing and onset is occurring in younger individuals with potential of a liver transplant epidemic in the future [7]. Therefore, diagnosis of NAFLD in its early stages in essential for improving outcomes and reducing potential burdens associated with late-stage liver disease.

\subsubsection{Diagnosis}

NAFLD and NASH are diagnosed by liver biopsy and histological evaluation of the liver for steatosis, inflammation, and hepatocellular ballooning, or enlargement of hepatocytes [54]. Histological grading for steatosis is divided into four scores: a score of 0 indicates no sign of fat infiltration, a score of 1 indicates fat infiltration in $<33 \%$ of hepatocytes, a score of 2 indicates fat infiltration in $33-66 \%$ of hepatocytes, and a score of 3 indicates fat infiltration in $>66 \%$ of hepatocytes [55]. Similarly, inflammation is categorized by grading. Inflammation grades are divided into lobular inflammation, chronic portal inflammation, and cell ballooning. Liver lobular inflammation is scored as: 0 being no sign of inflammation, 1 being $<2$ signs of lobular inflammation present, 2 being 2-4 signs of lobular inflammation present, and 3 being $>4$ signs of lobular inflammation present. Chronic portal inflammation is scored as a 0 , 1 , or 2, being none, mild, or severe respectively. Ballooning is scored similarly, with 0 being none, 1 being few, and 2 being many [54,56]. Fibrosis is scored as: 0 being no fibrosis, 1 being fibrosis in the presinusoidal or portal region, 2 being fibrosis in the presinusoidal and portal region, 3 being bridging of fibrosis, and 4 being cirrhosis [57].

While liver biopsies provide the most reliable method for diagnosing NAFLD and NASH, the procedure is invasive, costly, and only performed when an individual is suspected of having 
liver disease [58]. Researchers have criticized pitfalls in using liver biopsies and histology for diagnosis due to inability for early NAFLD detection and potential confounders causing liver damage separate from diet (i.e. alcohol and drugs) [59]. Therefore, other methods are also used to diagnosis liver disease. Serum measurement of alanine aminotransferase (ALT) and aspartate aminotransferase (AST) are used as biological indicators of liver injury [60]. ALT and AST are enzymes that catalyze reactions important for transamination and elevated levels can indicate liver damage [61]. However, studies have shown serum ALT and AST levels to be normal in up to $60 \%$ of individuals with NAFLD and NASH, indicating the value of these enzymes for diagnosing and are insufficient [62]. Other measurements used to complement ALT and AST measures include: insulin resistance using homeostatic model assessment (HOMA), fasting glucose, triglycerides, cholesterol, as well as blood pressure and abdominal circumference [57]. New imaging tools are being developed to diagnose NAFLD. Abdominal ultrasounds are being widely used to aid diagnosis of NAFLD; however, few studies have investigated their accuracy [63]. Therefore, histological evaluation remains 'the gold standard' for determining NAFLD and disease progression.

\subsubsection{Treatment}

In the absence of Food and Drug Administration approved drugs that specifically treat NAFLD, lifestyle changes are the primary treatment for liver disease $[64,65]$. Weight loss diet modification to achieve a healthier weight is recommended to treat NAFLD. Other dietary recommendations are to decrease saturated fat, sucrose, and fructose consumption. Decreasing overconsumption of saturated fat and simple sugars reduces DNL and inflammation [1]. Additionally, increased consumption of $n-3$ fatty acids, as well as complex carbohydrates, dietary fiber, and antioxidants have been suggested as treatments for NAFLD $[33,65,66]$. Increasing consumption of n-3 fatty acids, particularly eicosapentaenoic acid (EPA) and docosahexaenoic acid (DHA), has been shown to decrease expression of SREBP-1c and 
ChREBP resulting in decreased circulating free fatty acids, DNL and hepatic fat storage. Supplementing with long-chain n-3 fatty acids has also been shown to reduce inflammation and oxidative stress through modulation of inflammatory cytokines, particularly IL-6 and TNF- $\alpha$ [67]. Studies have yet to elucidate the optimal n-3 PUFA to treat NAFLD $[67,68]$.

Dietary fiber also has health benefits associated with reducing NAFLD. Soluble dietary fiber binds to LDL- and VLDL-cholesterol decreases circulating cholesterol $[69,70]$. Soluble fiber consumption also produces short-chain fatty acids. Short chain fatty acids have been shown to decrease hepatic inflammation, lipid deposition, and to increase lipid metabolism [71]. Dietary fiber also bind to bile acids resulting in a decrease in circulating bile acids, ameliorating increased bile acids caused by NAFLD [69,72]. Additionally, increasing antioxidant intake has been suggested for NAFLD since individuals with liver disease have been shown to have increased inflammation, ROS, and lipid peroxidation [4,25]. Antioxidants stabilize free radicals by donating electrons ameliorating the impact of free radicals on ROS and inflammation [73]. Although a plethora of studies have been conducted on various dietary antioxidants, results have produced inconsistences in regarding effectiveness [74-76]. Studies suggest antioxidants may have the greatest efficacy when combined with dietary fiber [75,77].

Studies have been conducted utilizing insulin sensitizing drugs, LDL-cholesterol reducing drugs, and bile acid targeting drugs have produced inconsistent results $[7,76,78,79]$. However, as discussed diet was able to favorably modulate the proposed drug targets for treating NAFLD [66]. Using functional food to treat of NAFLD has recently gained attention. Functional foods are defined as foods that beneficially affect one or more target functions in the body beyond adequate nutritional effects. Functional foods can be whole foods such as an apple or isolated components antioxidant compounds derived from apples [73]. Recent studies on the role of functional foods, particularly antioxidant and probiotic foods, as a treatment NAFLD have produced favorable results indicating potential for use as a treatment for NAFLD 
[80-84]. Although new drugs and physiological targets continue to be studied, lifestyle modification remains the standard for treating individuals diagnosed with NAFLD.

\subsubsection{Conclusions}

NAFLD is the most prevalent liver disease in the world, with projections estimating a potential liver disease epidemic [9,39]. NAFLD is primarily a lifestyle disease with increased saturated fat and simple carbohydrate consumption and physical inactivity central to disease etiology [4]. Onset of NAFLD is characterized by steatosis caused by alterations in lipid metabolism leading to increased DNL and alterations to bile acid homeostasis subsequently, followed by progression to NASH due to increased inflammation and ROS $[4,21,85]$. As NAFLD progresses to the more severe $\mathrm{NASH}$, the disease becomes more difficult to manage. Eventually, untreated NASH can lead to cirrhosis, an incurable liver disease requiring liver transplant [6]. The continual increase in NAFLD prevalence, with onset occurring at a younger age, gives credence to a potential liver transplant epidemic [9]. Therefore, improved diagnosis and treatment of NAFLD are essential.

Difficulties with diagnosing NAFLD has shifted focus to finding novel and effective treatments. Currently, no drugs are approved to treat NAFLD and therefore, lifestyle modification are primary treatment [64]. Dietary recommendations include decreasing saturated fat and simple carbohydrate intake and increasing complex carbohydrate and antioxidant intake [86]. Fruits, such as apples are high in dietary fiber and antioxidant compounds indicating its potential as a nutrition aid in the prevention and treatment of NAFLD $[87,88]$. Apple pomace, a waste byproduct from apple processing, should also be considered. When compared to apples, fiber content and antioxidant polyphenol content were higher in apple pomace [89,90]. 


\subsubsection{References}

1. Munteanu MA, Nagy GA, Mircea PA. Current management of NAFLD. Clujul Med. 2016;89(1):19-23. doi:10.15386/cjmed-539

2. Temple J, Cordero P, Li J, Nguyen V, Oben J. A Guide to Non-Alcoholic Fatty Liver Disease in Childhood and Adolescence. Int J Mol Sci. 2016;17(6):947.

3. Hashemi Kani A, Alavian SM, Esmaillzadeh A, Adibi P, Azadbakht L. Dietary Quality Indices and Biochemical Parameters Among Patients With Non Alcoholic Fatty Liver Disease (NAFLD). Hepat Mon. 2013;13(7):e10943. doi:10.5812/hepatmon.10943

4. Buzzetti E, Pinzani M, Tsochatzis EA. The multiple-hit pathogenesis of non-alcoholic fatty liver disease (NAFLD). Metabolism. 2016;65(8):1038-1048.

doi:10.1016/J.METABOL.2015.12.012

5. Ix JH, Sharma K. Mechanisms linking obesity, chronic kidney disease, and fatty liver disease: the roles of fetuin-A, adiponectin, and AMPK. J Am Soc Nephrol. 2010;21(3):406-412. doi:10.1681/ASN.2009080820

6. Michelotti GA, Machado M V., Diehl AM. NAFLD, NASH and liver cancer. Nat Rev Gastroenterol Hepatol. 2013;10(11):656-665. doi:10.1038/nrgastro.2013.183

7. Suzuki A, Diehl AM. Nonalcoholic Steatohepatitis. Annu Rev Med. 2017;68(1):85-98. doi:10.1146/annurev-med-051215-031109

8. Bellanti F, Villani R, Facciorusso A, Vendemiale G, Serviddio G. Lipid oxidation products in the pathogenesis of non-alcoholic steatohepatitis. Free Radic Biol Med. 2017;111:173-185. doi:10.1016/J.FREERADBIOMED.2017.01.023

9. Diehl AM, Day C. Cause, Pathogenesis, and Treatment of Nonalcoholic Steatohepatitis. Longo DL, ed. N Engl J Med. 2017;377(21):2063-2072.

10. Chalasani N, Younossi Z, Lavine JE, et al. The diagnosis and management of nonalcoholic fatty liver disease: Practice guidance from the American Association for the Study of Liver Diseases. Hepatology. 2018;67(1):328-357. doi:10.1002/hep.29367 
11. Mahady SE, George J. Predicting the future burden of NAFLD and NASH. J Hepatol. 2018;69(4):774-775. doi:10.1016/j.jhep.2018.06.025

12. Lim JS, Mietus-Snyder M, Valente A, Schwarz J-M, Lustig RH. The role of fructose in the pathogenesis of NAFLD and the metabolic syndrome. Nat Rev Gastroenterol Hepatol. 2010;7(5):251-264. doi:10.1038/nrgastro.2010.41

13. Younossi ZM, Stepanova M, Afendy M, et al. Changes in the Prevalence of the Most Common Causes of Chronic Liver Diseases in the United States From 1988 to 2008. Clin Gastroenterol Hepatol. 2011;9(6):524-530.e1. doi:10.1016/J.CGH.2011.03.020

14. Simopoulos AP. An increase in the omega-6/omega-3 fatty acid ratio increases the risk for obesity. Nutrients. 2016;8(3):128. doi:10.3390/nu8030128

15. Strable MS, Ntambi JM. Genetic control of de novo lipogenesis: role in diet-induced obesity. Crit Rev Biochem Mol Biol. 2010;45(3):199-214. doi:10.3109/10409231003667500 16. Naik A, Košir R, Rozman D. Genomic aspects of NAFLD pathogenesis. Genomics. 2013;102(2):84-95.

17. Wang X, Cao Y, Fu Y, Guo G, Zhang X. Liver fatty acid composition in mice with or without nonalcoholic fatty liver disease. Lipids Health Dis. 2011;10:234. doi:10.1186/1476-511X$10-234$

18. Millar JS, Stone SJ, Tietge UJF, et al. Short-term overexpression of DGAT1 or DGAT2 increases hepatic triglyceride but not VLDL triglyceride or apoB production. J Lipid Res. 2006;47(10):2297-2305. doi:10.1194/jr.M600213-JLR200

19. Peng XE, Wu YL, Lu QQ, Hu ZJ, Lin X. MTTP polymorphisms and susceptibility to nonalcoholic fatty liver disease in a Han Chinese population. Liver Int. 2014;34(1):118-128. 20. Alrefai WA, Gill RK. Bile Acid Transporters: Structure, Function, Regulation and Pathophysiological Implications. Pharm Res. 2007;24(10):1803-1823. doi:10.1007/s11095-007$9289-1$ 
21. Trauner M, Claudel T, Fickert P, Moustafa T, Wagner M. Bile acids as regulators of hepatic lipid and glucose metabolism. Dig Dis. 2010;28(1):220-224. doi:10.1159/000282091 22. Li T, Chiang JYL. Bile acid signaling in metabolic disease and drug therapy. Pharmacol Rev. 2014;66(4):948-983. doi:10.1124/pr.113.008201

23. Jiao N, Baker SS, Chapa-Rodriguez A, et al. Suppressed hepatic bile acid signalling despite elevated production of primary and secondary bile acids in NAFLD. Gut. 2018;67(10):1881-1891. doi:10.1136/gutjnl-2017-314307

24. Trauner M, Claudel T, Fickert P, Moustafa T, Wagner M. Bile acids as regulators of hepatic lipid and glucose metabolism. Dig Dis. 2010;28(1):220-224. doi:10.1159/000282091 25. Gambino R, Bugianesi E, Rosso C, et al. Different Serum Free Fatty Acid Profiles in NAFLD Subjects and Healthy Controls after Oral Fat Load. Int J Mol Sci. 2016;17(4):479. doi:10.3390/ijms17040479

26. Miyazaki M, Kim YC, Ntambi JM. A lipogenic diet in mice with a disruption of the stearoyl-CoA desaturase 1 gene reveals a stringent requirement of endogenous monounsaturated fatty acids for triglyceride synthesis. J Lipid Res. 2001;42(7):1018-1024. 27. van Dijk SJ, Feskens EJ, Bos MB, et al. A saturated fatty acid-rich diet induces an obesity-linked proinflammatory gene expression profile in adipose tissue of subjects at risk of metabolic syndrome. Am J Clin Nutr. 2009;90(6):1656-1664. doi:10.3945/ajcn.2009.27792 28. Milanski M, Degasperi G, Coope A, et al. Saturated fatty acids produce an inflammatory response predominantly through the activation of TLR4 signaling in hypothalamus: implications for the pathogenesis of obesity. J Neurosci. 2009;29(2):359-370.

doi:10.1523/JNEUROSCI.2760-08.2009

29. O'Neill LA., Kaltschmidt C. NF-kB: a crucial transcription factor for glial and neuronal cell function. Trends Neurosci. 1997;20(6):252-258.

30. Musso G. Non-alcoholic fatty liver, adipose tissue, and the bone: a new triumvirate on the block. Endocrine. 2012;42(2):237-239. doi:10.1007/s12020-012-9748-2 
31. Burhans MS, Ntambi JM. Monounsaturated Fatty Acid Mediated Liver-Adipose Tissue Crosstalk and Metabolic Regulation. In: Hepatic De Novo Lipogenesis and Regulation of Metabolism. Cham: Springer International Publishing; 2016:255-265. doi:10.1007/978-3-31925065-6_12

32. Tamura S, Shimomura I. Contribution of adipose tissue and de novo lipogenesis to nonalcoholic fatty liver disease. J Clin Invest. 2005;115(5):1139-1142. doi:10.1172/JCI24930 33. Trappoliere M, Tuccillo C, Federico A, et al. The treatment of NAFLD. Eur Rev Med Pharmacol Sci. 2005;9(5):299-304. http://www.ncbi.nlm.nih.gov/pubmed/16231594. Accessed January 25, 2019.

34. Araya J, Rodrigo R, Videla LA, et al. Increase in long-chain polyunsaturated fatty acid n $6 / n-3$ ratio in relation to hepatic steatosis in patients with non-alcoholic fatty liver disease. Clin Sci (Lond). 2004;106(6):635-643. doi:10.1042/CS20030326

35. Salem N, Pawlosky R, Wegher B, Hibbeln J. In vivo conversion of linoleic acid to arachidonic acid in human adults. Prostaglandins, Leukot Essent Fat Acids. 1999;60(5-6):407410. doi:10.1016/S0952-3278(99)80021-0

36. Sassa T, Kihara A. Metabolism of very long-chain Fatty acids: genes and pathophysiology. Biomol Ther (Seoul). 2014;22(2):83-92. doi:10.4062/biomolther.2014.017 37. Chilton F, Murphy R, Wilson B, et al. Diet-Gene Interactions and PUFA Metabolism: A Potential Contributor to Health Disparities and Human Diseases. Nutrients. 2014;6(5):19932022. doi:10.3390/nu6051993

38. Abbott SK, Else PL, Atkins TA, Hulbert AJ. Fatty acid composition of membrane bilayers: Importance of diet polyunsaturated fat balance. Biochim Biophys Acta - Biomembr. 2012;1818(5):1309-1317. doi:10.1016/J.BBAMEM.2012.01.011

39. Loomba R, Sanyal AJ. The global NAFLD epidemic. Nat Rev Gastroenterol Hepatol. 2013;10(11):686-690. doi:10.1038/nrgastro.2013.171 
40. Basaranoglu M, Basaranoglu G, Bugianesi E. Carbohydrate intake and nonalcoholic fatty liver disease: fructose as a weapon of mass destruction. Hepatobiliary Surg Nutr. 2015;4(2):109-116. doi:0.3978/j.issn.2304-3881.2014.11.05

41. Mosca A, Nobili V, De Vito R, et al. Serum uric acid concentrations and fructose consumption are independently associated with $\mathrm{NASH}$ in children and adolescents. J Hepatol. 2017;66(5):1031-1036. doi:10.1016/J.JHEP.2016.12.025

42. Giordano C, Karasik O, King-Morris K, Asmar A. Uric Acid as a Marker of Kidney Disease: Review of the Current Literature. Dis Markers. 2015;2015:382918. doi:10.1155/2015/382918

43. Byrne CD, Targher G. NAFLD: A multisystem disease. J Hepatol. 2015;62(1):S47-S64. doi:10.1016/J.JHEP.2014.12.012

44. Mantovani A, Ballestri S, Lonardo A, Targher G. Cardiovascular Disease and Myocardial Abnormalities in Nonalcoholic Fatty Liver Disease. Dig Dis Sci. 2016;61(5):1246-1267. doi:10.1007/s10620-016-4040-6

45. Cui R, Sheng H, Rui X-F, et al. Low bone mineral density in chinese adults with nonalcoholic Fatty liver disease. Int J Endocrinol. 2013;2013:396545. doi:10.1155/2013/396545 46. Wang Y, Wen G, Zhou R, et al. Association of Nonalcoholic Fatty Liver Disease With Osteoporotic Fractures: A Cross-Sectional Retrospective Study of Chinese Individuals. Front Endocrinol (Lausanne). 2018;9:408. doi:10.3389/fendo.2018.00408

47. Filip R, Radzki RP, Bieńko M. Novel insights into the relationship between nonalcoholic fatty liver disease and osteoporosis. Clin Interv Aging. 2018;13:1879-1891. doi:10.2147/CIA.S170533

48. Tilg $\mathrm{H}$, Moschen A. Weight loss: cornerstone in the treatment of non-alcoholic fatty liver disease. Minerva Gastroenterol Dietol. 2010;56(2):159-167. http://www.ncbi.nlm.nih.gov/pubmed/20485253. Accessed January 25, 2019. 
49. Schuppan D, Afdhal NH. Liver cirrhosis. Lancet. 2008;371(9615):838-851.

doi:10.1016/S0140-6736(08)60383-9

50. Mokdad AA, Lopez AD, Shahraz S, et al. Liver cirrhosis mortality in 187 countries between 1980 and 2010: a systematic analysis. BMC Med. 2014;12(1):145.

doi:10.1186/s12916-014-0145-y

51. Zeng M De, Fan JG, Lu LG, et al. Guidelines for the diagnosis and treatment of nonalcoholic fatty liver diseases. J Dig Dis. 2008;9(2):108-112. doi:10.1111/j.1751-

2980.2008.00331.x

52. Farrell GC, Larter CZ. Nonalcoholic fatty liver disease: From steatosis to cirrhosis. Hepatology. 2006;43(S1):S99-S112. doi:10.1002/hep.20973

53. Collier JD, Ninkovic M, Compston JE. Guidelines on the management of osteoporosis associated with chronic liver disease. Gut. 2002;50(suppl 1):i1-i9.

doi:10.1136/GUT.50.SUPPL_1.I1

54. Brunt EM, Kleiner DE, Wilson LA, Belt P, Neuschwander-Tetri BA. Nonalcoholic fatty liver disease (NAFLD) activity score and the histopathologic diagnosis in NAFLD: distinct clinicopathologic meanings. Hepatology. 2011;53(3):810-820. doi:10.1002/hep.24127

55. Kleiner DE, Brunt EM, Van Natta M, et al. Design and validation of a histological scoring system for nonalcoholic fatty liver disease. Hepatology. 2005;41(6):1313-1321.

doi:10.1002/hep.20701

56. Kleiner DE, Behling C, Brunt EM, et al. Comparison of adult and pediatric NAFLDconfirmation of a second pattern of progressive fatty liver disease in children: 189. Hepatology. 2006;44:259A-260A. https://insights.ovid.com/hepatology/hepa/2006/10/001/comparison-adultpediatric-nafld-confirmation/189/01515467.

57. Angulo P, Hui JM, Marchesini G, et al. The NAFLD fibrosis score: A noninvasive system that identifies liver fibrosis in patients with NAFLD. Hepatology. 2007;45(4):846-854.

doi:10.1002/hep.21496 
58. Bedossa P, Carrat F. Liver biopsy: the best, not the gold standard. J Hepatol. 2009;50(1):1-3. doi:10.1016/j.jhep.2008.10.014

59. Koch LK, Yeh MM. Nonalcoholic fatty liver disease (NAFLD): Diagnosis, pitfalls, and staging. Ann Diagn Pathol. 2018;37:83-90. doi:10.1016/J.ANNDIAGPATH.2018.09.009

60. lacobellis A, Marcellini M, Andriulli A, et al. Non invasive evaluation of liver fibrosis in paediatric patients with nonalcoholic steatohepatitis. World J Gastroenterol. 2006;12(48):78217825.

61. Qian K, Zhong S, Xie K, Yu D, Yang R, Gong D-W. Hepatic ALT isoenzymes are elevated in gluconeogenic conditions including diabetes and suppressed by insulin at the protein level. Diabetes Metab Res Rev. 2015;31(6):562-571. doi:10.1002/dmrr.2655

62. Manco M, Alisi A, Nobili V. Risk of severe liver disease in NAFLD with normal ALT levels: A pediatric report. Hepatology. 2008;48(6):2087-2088. doi:10.1002/hep.22631 63. Mishra P, Younossi ZM. Abdominal Ultrasound for Diagnosis of Nonalcoholic Fatty Liver Disease (NAFLD). Am J Gastroenterol. 2007;102(12):2716-2717. doi:10.1111/j.15720241.2007.01520.x

64. Hannah WN, Harrison SA. Lifestyle and Dietary Interventions in the Management of Nonalcoholic Fatty Liver Disease. Dig Dis Sci. 2016;61(5):1365-1374.

65. LaBrecque DR, Abbas Z, Anania F, et al. World Gastroenterology Organisation Global Guidelines. J Clin Gastroenterol. June 2014:1. doi:10.1097/MCG.0000000000000116 66. Romero-Gómez M, Zelber-Sagi S, Trenell M. Treatment of NAFLD with diet, physical activity and exercise. J Hepatol. 2017;67(4):829-846. doi:10.1016/J.JHEP.2017.05.016 67. Scorletti E, Byrne CD. Omega-3 fatty acids and non-alcoholic fatty liver disease: Evidence of efficacy and mechanism of action. Mol Aspects Med. 2018;64:135-146. doi:10.1016/J.MAM.2018.03.001 
68. Parker HM, Johnson NA, Burdon CA, Cohn JS, O’Connor HT, George J. Omega-3 supplementation and non-alcoholic fatty liver disease: A systematic review and meta-analysis. $\mathrm{J}$ Hepatol. 2012;56(4):944-951. doi:10.1016/J.JHEP.2011.08.018

69. Anderson JW, Baird P, Davis Jr RH, et al. Health benefits of dietary fiber. Nutr Rev. 2009;67(4):188-205. doi:10.1111/j.1753-4887.2009.00189.x

70. Reimer RA, Grover GJ, Koetzner L, Gahler RJ, Lyon MR, Wood S. The soluble fiber complex PolyGlycopleX lowers serum triglycerides and reduces hepatic steatosis in highsucrose-fed rats. Nutr Res. 2011;31(4):296-301. doi:10.1016/J.NUTRES.2011.03.012

71. McNabney S, Henagan T, McNabney SM, Henagan TM. Short Chain Fatty Acids in the Colon and Peripheral Tissues: A Focus on Butyrate, Colon Cancer, Obesity and Insulin Resistance. Nutrients. 2017;9(12):1348. doi:10.3390/nu9121348

72. Kern F, Birkner HJ, Ostrower VS. Binding of bile acids by dietary fiber. Am J Clin Nutr. 1978;31(10):S175-S179. doi:10.1093/ajcn/31.10.S175

73. Lobo V, Patil A, Phatak A, Chandra N. Free radicals, antioxidants and functional foods: Impact on human health. Pharmacogn Rev. 2010;4(8):118-126. doi:10.4103/0973-7847.70902 74. Madan K, Bhardwaj P, Thareja S, Gupta SD, Saraya A. Oxidant Stress and Antioxidant Status Among Patients With Nonalcoholic Fatty Liver Disease (NAFLD). J Clin Gastroenterol. 2006;40(10):930-935. doi:10.1097/01.mcg.0000212608.59090.08

75. Chang CY, Argo CK, Al-Osaimi AMS, Caldwell SH. Therapy of NAFLD: antioxidants and cytoprotective agents. J Clin Gastroenterol. 2006;40 Suppl 1:S51-60.

doi:10.1097/01.mcg.0000168648.79034.67

76. Adams LA, Angulo P. Treatment of non-alcoholic fatty liver disease. Postgrad Med J. 2006;82(967):315-322. doi:10.1055/s-2001-12931

77. Vuksan V, Sievenpiper JL, Owen R, et al. Beneficial effects of viscous dietary fiber from Konjac-mannan in subjects with the insulin resistance syndrome: results of a controlled metabolic trial. Diabetes Care. 2000;23(1):9-14. doi:10.2337/DIACARE.23.1.9 
78. Luef G, Rauchenzauner M, Waldmann M, et al. Non-alcoholic fatty liver disease (NAFLD), insulin resistance and lipid profile in antiepileptic drug treatment. Epilepsy Res. 2009;86(1):42-47. doi:10.1016/J.EPLEPSYRES.2009.04.004

79. Zhu L, Baker RD, Zhu R, Baker SS. Bile Acids and the Gut Microbiome as Potential Targets for NAFLD Treatment. J Pediatr Gastroenterol Nutr. 2018;67(1):3-5. doi:10.1097/MPG.0000000000002010

80. Sakata R, Nakamura T, Torimura T, Ueno T, Sata M. Green tea with high-density catechins improves liver function and fat infiltration in non-alcoholic fatty liver disease (NAFLD) patients: A double-blind placebo-controlled study. Int J Mol Med. 2013;32(5):989-994 . doi:10.3892/jmm.2013.1503

81. Duric M, Sivanesan S, Bakovic M. Phosphatidylcholine functional foods and nutraceuticals: A potential approach to prevent non-alcoholic fatty liver disease. Eur J Lipid Sci Technol. 2012;114(4):389-398. doi:10.1002/ejlt.201100350

82. Chao J, Huo T-I, Cheng H-Y, et al. Gallic Acid Ameliorated Impaired Glucose and Lipid Homeostasis in High Fat Diet-Induced NAFLD Mice. Luque RM, ed. PLoS One. 2014;9(6):e96969. doi:10.1371/journal.pone.0096969

83. Iacono A, Raso GM, Canani RB, Calignano A, Meli R. Probiotics as an emerging therapeutic strategy to treat NAFLD: focus on molecular and biochemical mechanisms. J Nutr Biochem. 2011;22(8):699-711. doi:10.1016/J.JNUTBIO.2010.10.002

84. Morisco F, Vitaglione P, Amoruso D, Russo B, Fogliano V, Caporaso N. Foods and liver health. Mol Aspects Med. 2008;29(1-2):144-150. doi:10.1016/J.MAM.2007.09.003

85. Reddy JK, Sambasiva Rao M. Lipid Metabolism and Liver Inflammation. II. Fatty liver disease and fatty acid oxidation. Am J Physiol Liver Physiol. 2006;290(5):G852-G858. doi:10.1152/ajpgi.00521.2005

86. Kargulewicz A, Stankowiak-Kulpa H, Grzymisławski M. Dietary recommendations for patients with nonalcoholic fatty liver disease. Prz Gastroenterol. 2014;9(1):18-23. 
87. Boyer J, Liu RH. Apple phytochemicals and their health benefits. Nutr J. 2004;3(1):5. 88. Hyson DA. A comprehensive review of apples and apple components and their relationship to human health. Adv Nutr. 2011;2(5):408-420. doi:10.3945/an.111.000513 89. Bhushan S, Kalia K, Sharma M, Singh B, Ahuja PS. Processing of apple pomace for bioactive molecules. Crit Rev Biotechnol. 2008;28(4):285-296.

doi:10.1080/07388550802368895

90. Gazalli $\mathrm{H}$, Malik $\mathrm{AH}$, Sofi $\mathrm{AH}$, et al. Nutritional value and physiological effect of apple pomace. Int J Food Nutr Saf. 2014;5(1):11-15.

91. Browning JD, Horton JD. Molecular mediators of hepatic steatosis and liver injury. J Clin Invest. 2004;114(2):147-152. doi:10.1172/JCI22422

92. Giorgio V, Prono F, Graziano F, Nobili V. Pediatric non alcoholic fatty liver disease: old and new concepts on development, progression, metabolic insight and potential treatment targets. BMC Pediatr. 2013;13:40. doi:10.1186/1471-2431-13-40 


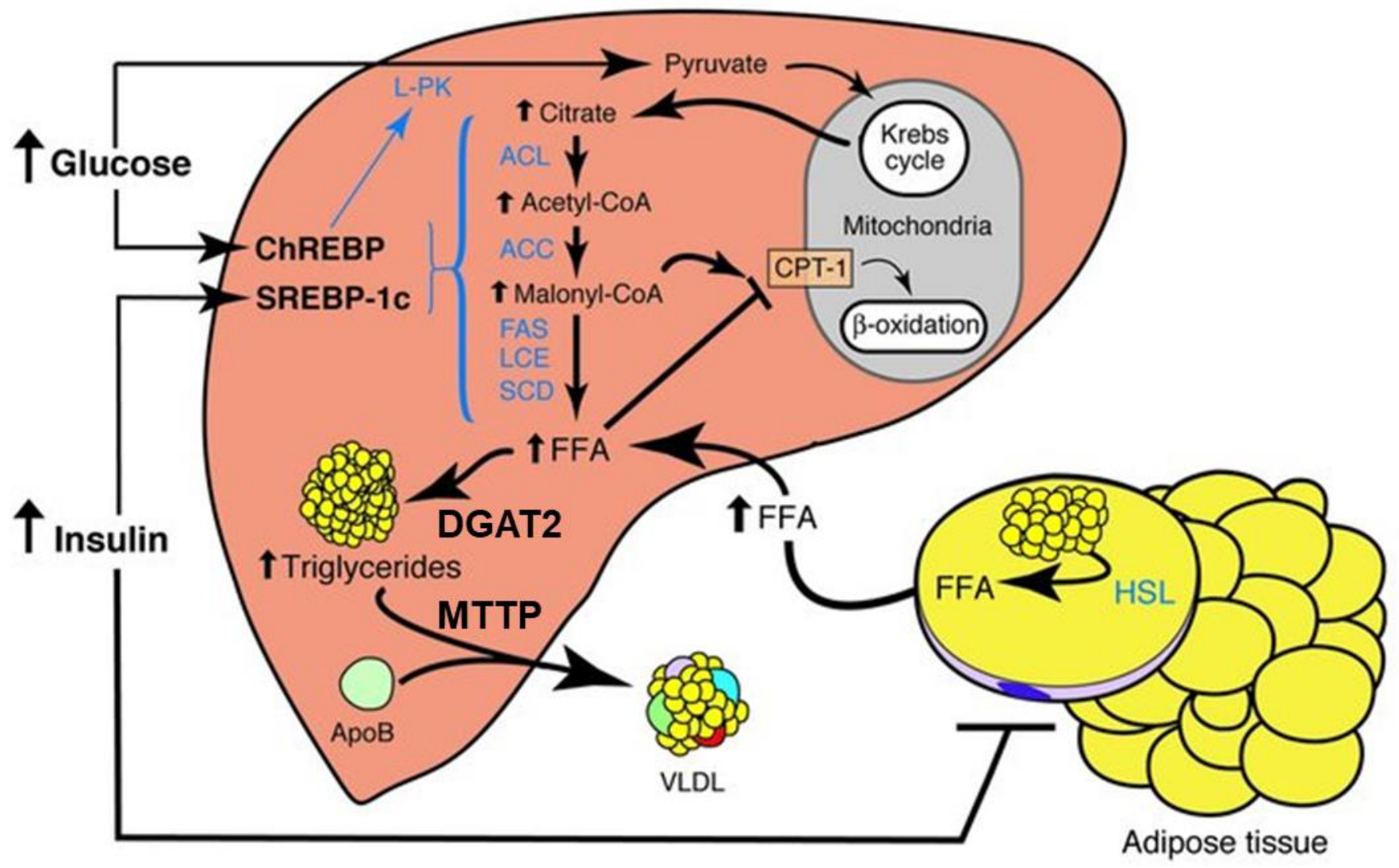

Figure 1. Illustration of de novo lipogenesis, lipid storage, and transport pathway. Adapted from Browning, et al [91]. Abbreviations: ACC, acetyl co-A carboxylase; ACL, ATP citrate synthase; ApoB, apolipoprotein B; ChREBP, carbohydrate response element bind protein; CPT-1, carnitine palmitoyltransferase 1; diacylglycerol O-acyltransferase 2; FAS, fatty acid synthase; FFA, free fatty acids; HSL, hormone sensitive lipase; LCE, long chain fatty acyl elongase; SCD, stearoyl-CoA desaturase; SREBP1c; sterol regulatory element binding protein 1c; VLDL, very low-density lipoprotein [6]. 
First Hit
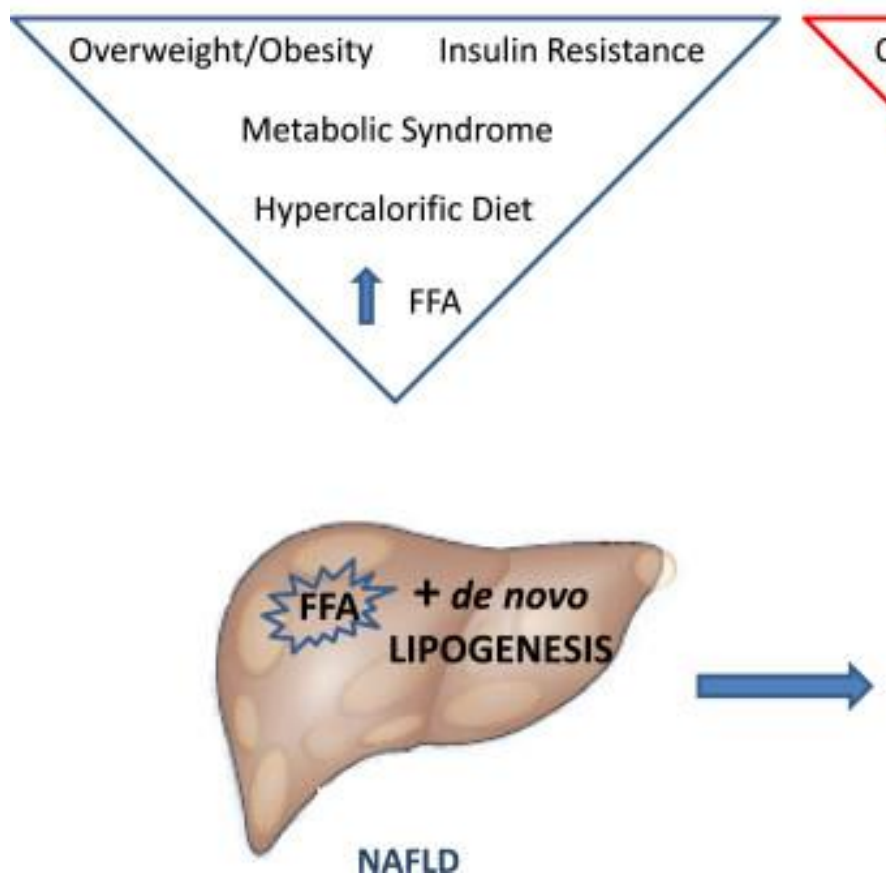

Second Hit
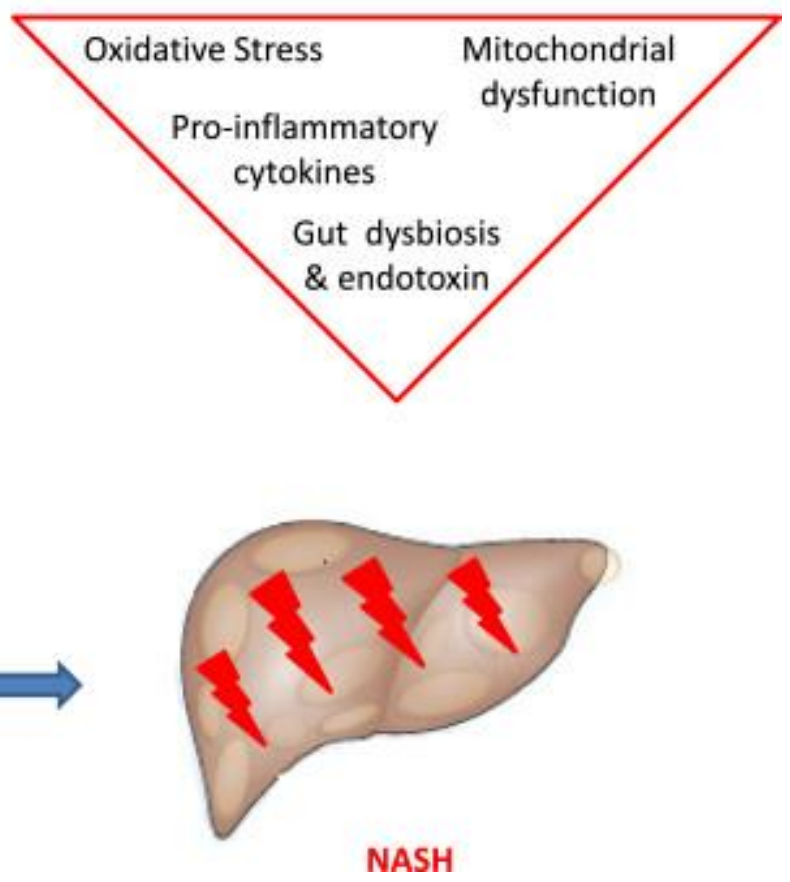

Figure 2. Depiction of the multiple hit theory of liver disease progression. Abbreviations: FFA, free fatty acids; NAFLD, non-alcoholic fatty liver disease; NASH, non-alcoholic steatohepatitis [92]. 


\subsection{Study Objectives and Hypotheses Study 1}

Objective: To investigate the effect of $10 \%$ caloric substitution with apple pomace on features of Western diet-induced non-alcoholic fatty liver disease in growing female rats.

Hypothesis: Caloric substitution with apple pomace will attenuate features of non-alcoholic fatty liver disease in growing female rats consuming a Western diet.

\section{Study 2}

Objective: To determine the effect of $10 \%$ caloric substitution with apple pomace on features of Western diet-induced non-alcoholic steatohepatitis in growing female rats.

Hypothesis: Caloric substitution with apple pomace will attenuate features of Western dietinduced non-alcoholic steatohepatitis in growing female rats.

\section{Study 3}

Objectives: To determine the effect of $10 \%$ caloric substitution with apple pomace on renal and bone health in growing female rats consuming 'healthy' and Western diets.

Hypothesis: Caloric substitution with apple pomace will not have detriment renal or bone health in growing female rats consuming 'healthy' or Western diets.

Overall Hypothesis: A 10\% caloric substitution with apple pomace will attenuate Western dietinduced onset of non-alcoholic fatty liver disease and progression to non-alcoholic steatohepatitis and will be safe for consumption, regardless of diet quality in growing female rats. 
Apple pomace consumption favorably alters hepatic lipid metabolism in young female Sprague-Dawley rats fed a Western diet

R. Chris Skinner ${ }^{1}$, Derek C. Warren ${ }^{1}$, Soofia N. Lateef ${ }^{2}$, Vagner A. Benedito ${ }^{3}$, Janet C. Tou ${ }^{\star *}$

${ }^{1}$ Division of Animal and Nutritional Sciences, West Virginia University, Morgantown, WV 26506;

rcskinner@mix.wvu.edu, dwarren2@mix.wvu.edu

²Department of Chemical Engineering, West Virginia University, Morgantown, WV 26506;

snlateef@mix.wvu.edu

${ }^{3}$ Division of Plant and Soil Sciences, West Virginia University, Morgantown, WV 26506;

vagner.benedito@mail.wvu.edu

*Correspondence: janet.tou@mail.wvu.edu; Tel.: +1-304-293-1919

Published in Nutrients in November 2018 as: Skinner R, Warren D, Lateef S, et al. Apple Pomace Consumption Favorably Alters Hepatic Lipid Metabolism in Young Female SpragueDawley Rats Fed a Western Diet. Nutrients. 2018;10(12):1882. doi:10.3390/nu10121882 


\subsection{Abstract}

Apple pomace, a waste byproduct of processing, is rich in several nutrients, particularly dietary fiber, indicating potential benefits for diseases attributed to poor diets, such as nonalcoholic fatty liver disease (NAFLD). NAFLD affects over $25 \%$ of United States population and is increasing in children. Increasing fruit consumption can decrease NAFLD. The study objective was to replace calories in standard or Western diets with apple pomace to determine effects on genes regulating hepatic lipid metabolism and on risk of NAFLD. Female Sprague-Dawley rats were randomly assigned ( $n=8$ rats/group) to isocaloric diets of $A I N-93 G$ and AIN-93G/10\% w/w apple pomace (AIN/AP) or isocaloric diets of Western (45\% fat, 33\% sucrose) and Western/10\% w/w apple pomace (Western/AP) diets for 8 weeks. There were no significant effects on hepatic lipid metabolism in rats fed AIN/AP. Western/AP diet containing fiber-rich apple pomace attenuated fat vacuole infiltration, elevated monounsaturated fatty acid content, and triglyceride storage in the liver due to higher circulating bile and upregulated hepatic DGAT2 gene expression induced by feeding a Western diet. The study results showed replacement of calories in Western diet with apple pomace attenuated NAFLD risk. Therefore, apple pomace has potential to be developed into a sustainable functional food for human consumption.

Keywords: apple pomace, NAFLD, Western diet, DGAT2, bile acids, food waste, sustainability 


\subsection{Introduction}

One-third of apples harvested in the United States (USA) are processed into apple products [1]. Apple pomace is a byproduct of apple processing that includes: skin, stem, seeds, core, and calyx [2]. Apple pomace presents an environmental and public health issue due to its rapid spoilage and fermentation. Moreover, apple pomace disposal is expensive with annual costs being estimated at $\$ 10$ million in the USA $[3,4]$. Yet, apple pomace is a rich source of various nutrients (e.g., phytochemicals, vitamins, dietary minerals), but it is particularly high in non-digestible carbohydrates and dietary fibers, indicating potential benefits for reducing metabolic dysfunction, such as non-alcoholic fatty liver disease (NAFLD) [5].

NAFLD is characterized by dysregulated lipid metabolism and liver steatosis. It is the most prevalent liver disease worldwide with reports of over $25 \%$ of the population having NAFLD [6]. Global prevalence in children is estimated to be between 7.6-34.2\% [7]. Further, liver steatosis has also been diagnosed in non-obese patients [8]. Diets that are high in fat and sucrose, which characterize Western diets, have been shown to induce NAFLD [9,10]. High carbohydrate consumption has been linked to NAFLD progression by upregulating the expression of key gene transcription factors that are involved in hepatic de novo lipogenesis (DNL), such as sterol regulatory element-binding protein-1c (SREBP-1c) and carbohydrate response element binding protein (ChREBP) [11]. SREBP-1c and ChREBP stimulate fatty acid synthase (FAS) to catalyze the synthesis of saturated fatty acids (SFAs). In turn, SFAs can be desaturated to monounsaturated fatty acids (MUFAs) by stearoyl-CoA desaturase-1 (SCD-1). Promoting fatty acid esterification by diacylglycerol O-acyl transferase-2 (DGAT2) stimulates hepatic triglyceride synthesis that can contribute to hepatic steatosis and production of triglyceride-rich very low density lipoproteins (VLDLs), which characterizes NAFLD [12].

Currently, there are no approved drugs for the treatment of NAFLD; therefore, management of NAFLD relies on proper diet and lifestyle changes [13]. Despite a paucity of studies, apple pomace has been shown to reduce metabolic risk factors, such as hyperglycemia 
and dyslipidemia, providing rationale for apple pomace consumption to reduce NAFLD [14,15]. However, a potential concern is apple pomace's fructose content. Excessive fructose consumption has been shown to promote NAFLD development and progression [16]. Previously, we reported growing female Sprague-Dawley rats consuming different fructose containing drinks for eight weeks promoted NAFLD, but had no significant effect on body weight when compared to the water control [17]. Therefore, the objectives of this study were to determine the effects of caloric substitution with apple pomace in a normal (standard diet) or Western diet on expression of genes regulating hepatic lipid metabolism and NAFLD risk in a rat model. Our study followed the suggested dietary advice of replacing calories in the diet with healthier food choices instead of dietary supplementation with a purified isolated nutrient [18]. Study results showed that apple pomace had no detrimental effects on hepatic lipid metabolism and liver health in rats consuming normal diets and attenuate features in the NAFLD spectrum of upregulated gene expression of triglyceride synthesis as well as liver steatosis induced in rats consuming a Western diet. Investigating whether apple pomace, a byproduct generated from apple processing, can be re-purposed as a functional food for human consumption has the potential to improve public health and food sustainability by providing an economical solution for reducing environmental pollution and costly waste disposal.

\subsection{Materials and Methods}

\section{Animals and Diets}

Weanling (age 22-29 days) female Sprague-Dawley rats $(n=32)$ were purchased from Harlan-Teklad (Indianapolis, IN, USA). Female rats were selected on the basis of their greater susceptibility to hepatic effects with increased carbohydrate consumption [19]. All animal procedures were approved by the Animal Care and Use Committee at West Virginia University and were conducted in accordance with the guidelines of the National Research Council for the Care of Laboratory Animals [20]. Rats were individually housed with cages kept in a room at constant temperature of $21 \pm 2^{\circ} \mathrm{C}$ with a $12 \mathrm{~h}$ light/dark cycle throughout the study. 
Following seven-days acclimation, rats were randomly assigned ( $\mathrm{n}=8$ rats/group) to four dietary groups consisting of: (1) AIN-93G, a standard purified rodent diet (AIN), (2) AIN-93G with $10 \%(\mathrm{~g} / \mathrm{kg})$ calorically substituted with apple pomace (AIN/AP), (3) Western diet (45\% fat, $33 \%$ sucrose by kcals), or (4) Western diet with $10 \%(\mathrm{~g} / \mathrm{kg})$ calorically substituted with apple pomace (Western/AP). AIN diets were adjusted to be isocaloric $(3.7-3.8 \mathrm{kcal} / \mathrm{g})$ and Western diets were adjusted to be isocaloric $(4.7 \mathrm{kcal} / \mathrm{g})$, resulting in different types rather than amounts of simple and complex carbohydrates. Detailed ingredient composition of experimental diets is provided in Table 1. Locally sourced apple pomace was provided by Swilled Dog Hard Cider Company (Franklin, WV, USA) and nutrient composition analysis was performed by Medallion Laboratories (Minneapolis, MN, USA) (Table 2). Total polyphenols in apple pomace and treatment diets were determined while using the Folin-Ciocalteu method [21]. Diets were stored at $-20^{\circ} \mathrm{C}$ until fed. Rats were provided ad libitum access to their assigned diets and deionized distilled water $\left(\mathrm{ddH}_{2} \mathrm{O}\right)$ throughout the eight weeks study duration. At baseline (day 1) and final (end of eight weeks), rats were individually housed in a metabolic cage for $24 \mathrm{~h}$ to collect feces. Feces was weighed and dried. Food intake was measured and assigned diets were replaced every other day while $\mathrm{ddH}_{2} \mathrm{O}$ was replaced weekly. Rats were fasted overnight then euthanized by carbon dioxide inhalation. The liver was excised, perfused with $0.7 \%$ saline solution, weighed, and then flash frozen in liquid nitrogen. Rat livers were stored at $-80^{\circ} \mathrm{C}$ until analyzed.

\section{Liver total lipid and triglyceride content}

Lipid extraction was performed according to Bligh and Dyer [22]. Briefly, $1 \mathrm{~g}$ of liver tissue was homogenized in Tris/EDTA buffer ( $\mathrm{pH}$ 7.4). To quantify fatty acids, $50 \mu \mathrm{L}$ of nonadecanoic acid (19:0) was added as a standard during the initial weighing of the samples. A chloroform: methanol:acetic acid $(2: 1: 0.15, \mathrm{v} / \mathrm{v} / \mathrm{v})$ solution was added to liver samples, centrifuged at $900 \times \mathrm{g}$ for $10 \mathrm{~min}$ at $10^{\circ} \mathrm{C}$, and the bottom chloroform layer collected. The collected chloroform layer 
was mixed with chloroform: methanol $(4: 1, \mathrm{v} / \mathrm{v})$ and centrifuged at $900 \times \mathrm{g}$ at $10^{\circ} \mathrm{C}$ for $10 \mathrm{~min}$. The chloroform layer was then collected and filtered. Extracted lipids were dried under nitrogen gas. Total lipid content in the liver was gravimetrically determined.

Liver triglyceride content was determined using a commercially available triglyceride colorimetric assay kit (Cayman Chemicals, Ann Arbor, MI, USA). Briefly, liver tissue (400 mg) was homogenized using assay standard diluent $(2 \mathrm{~mL})$. Tissue homogenate was centrifuged at $10,000 \times \mathrm{g}$ for $10 \mathrm{~min}$ at $4^{\circ} \mathrm{C}$. Supernatant was collected and diluted $1: 5$ in assay standard diluent. Hepatic samples $(10 \mu \mathrm{L})$ and lipase enzyme solution (150 $\mu \mathrm{L})$ were added to a 96-well cell culture plate and then incubated for $15 \mathrm{~min}$. Hepatic sample absorbance was measured at 540 nm using a BioTek Epoch microplate spectrophotometer (Winooski, VT, USA). All samples were performed in duplicate. The inter-assay coefficient of variation was $15.16 \%$.

\section{Diet and liver fatty acid composition}

Following lipid extraction, diet and liver tissue samples were transmethylated according to the method described by Fritsche and Johnston [23]. Briefly, fatty acids were methylated by adding $4 \%$ sulfuric acid in anhydrous methanol to the extracted lipid samples followed by incubation in a $90^{\circ} \mathrm{C}$ water bath for $60 \mathrm{~min}$. Samples were cooled to room temperature and $\mathrm{ddH}_{2} \mathrm{O}$ was added. Chloroform was then added to the methylated samples and centrifuged at $900 \times \mathrm{g}$ for $10 \mathrm{~min}$ at $10^{\circ} \mathrm{C}$. The collected chloroform layer was filtered through anhydrous sodium sulfate to remove any remaining water. Fatty acid methyl esters (FAMEs) were dried under nitrogen gas and re-suspended in iso-octane.

FAMEs were analyzed by gas liquid chromatography (CP-3800; Varian, Walnut Creek, CA, USA) using an initial temperature of $140^{\circ} \mathrm{C}$ held for 5 min and then increased $1^{\circ} \mathrm{C}$ per min to a final temperature of $220^{\circ} \mathrm{C}$. A wall-coated open tubular fused silica capillary column (Varian, Walnut Creek, CA) was used to separate FAME with CP-Sil 88 at the stationary phase. Nitrogen was used as the carrier gas and the total separation time was 56 min. Quantitative 37 
Component FAMEs Sigma Mix (Supelco, Bellefonte, PA, USA) was used to identify fatty acids. Fatty acids were determined by retention time and quantified using peak ultra-counts. All samples were performed in duplicate and reported as \% of total fatty acids.

\section{Liver histology}

The left lateral liver lobe $(n=7-8)$ was removed and immediately fixed in $10 \%$ buffered formalin solution for histological evaluation. Tissues were dehydrated through a series of increasing ethanol concentrations $\left(70-100 \%\right.$ in $\left.\mathrm{ddH}_{2} \mathrm{O}\right)$, then placed in xylene and embedded in paraffin. Sections $(8 \mu \mathrm{m})$ from each block were stained with hematoxylin and eosin. All slides were analyzed under a Nikon TE 2000-S light microscope (Nikon Instruments, Melville, NY, USA) by three trained individuals who were blinded to diet treatments. Liver fat accumulation was graded using the classification described by Brunt, et al., where grade 0 is no evidence of fat vacuoles, grade 1 is evidence of fat vacuoles in $<33 \%$ of hepatocytes, grade 2 is evidence of fat vacuoles in 33-66\% of hepatocytes, and grade 3 is evidence of fat vacuoles in $>66 \%$ of hepatocytes [24]. Images were captured using a PC interface with Q-Capture imaging software (Quantitative imaging Corporation, BC, Canada).

\section{RNA isolation and gene expression}

Total RNA was extracted from frozen tissue $(50 \mathrm{mg})$ using the Zymo Research mRNA Isolation Kit (Irvine, CA, USA) according to the manufacturer's instruction for total RNA isolation. Isolated RNA integrity was visualized on a $1.5 \%$ agarose gel and then quantified by spectrophotometry (NanoDrop 100; Thermo Scientific, Waltham, MA, USA). Following DNase I treatment with TURBO DNA-free kit (Applied Biosystems, Foster City, CA, USA), total mRNA was amplified using the Superscript III First-Strand Synthesis System with oligo dT primers (Invitrogen, Carlsbad, CA, USA). 
Real-time quantitative polymerase chain reaction (RT-qPCR) consisted of $2.5 \mu \mathrm{L}$ of SYBR Green Master Mix (Applied Biosystems, Foster City, CA, USA ), $1 \mu \mathrm{L}$ of cDNA (diluted 1:10), $1 \mu \mathrm{L}$ of forward and reverse primer solutions (10 $\mu \mathrm{M}$ each), and $0.5 \mu \mathrm{L}$ of deionized distilled water for a total reaction volume of $5 \mu \mathrm{L}$. The thermal profile consisted of $50{ }^{\circ} \mathrm{C}$ for 2 $\min , 95^{\circ} \mathrm{C}$ for $10 \mathrm{~min}$, and then 40 cycles of $95^{\circ} \mathrm{C}$ for $15 \mathrm{~s}$ and $60^{\circ} \mathrm{C}$ for $1 \mathrm{~min}$. A melt curve analysis was applied at the end of cycling. Primers were designed for ChREBP, SREBP-1c, sterol regulatory binding protein 2 (SREBP2), FAS, SCD-1, peroxisome proliferator-activated receptor- $\alpha$ (PPAR $\alpha$ ), peroxisome proliferator-activated receptor- $\gamma$ (PPAR $\gamma)$, hormone sensitive lipase (HSL), microsomal triglyceride transfer protein (MTTP), and DGAT2, as well as for housekeeping genes, $\beta$-actin and glyceraldehyde 2-phosphate dehydrogenase (GAPDH) using the Primer3 program (Howard Hughes Medical Institute) and respective mRNA sequences that were obtained from the NCBI database. Forward and reverse primers for gene transcriptions can be found in Appendix A.

\section{Serum biochemical measurements}

Rats were fasted overnight and euthanized by carbon dioxide inhalation. Blood was collected by aorta puncture. Collected blood was centrifuged at $1500 \times \mathrm{g}$ for $10 \mathrm{~min}$ at $4^{\circ} \mathrm{C}$ to obtain serum. Serum samples were stored at $-80^{\circ} \mathrm{C}$ until analyzed. Serum measures of liver function included: alanine aminotransferase (ALT) and aspartate aminotransferase (AST). Values were determined enzymatically using a commercially available Vet-16 rotor and were quantified by a Hemagen Analyst automated spectrophotometer (Hemagen Diagnostics Inc., Columbia, MD, USA). AST: ALT ratio was determined by dividing AST values by ALT values. Serum cholesterol, low-density lipoprotein-cholesterol (LDL-C)/VLDL, and high-density lipoprotein-cholesterol (HDL-C) were determined by commercially available fluorometric assay (Cell Biolabs, San Diego, CA, USA). Briefly, $200 \mu \mathrm{L}$ precipitation reagent was added to $200 \mu \mathrm{L}$ of serum and then centrifuged at $2000 \times \mathrm{g}$ for $20 \mathrm{~min}$. The supernatant containing HDL-C was 
removed and diluted to a final volume of $1: 50$. Pelleted portion containing LDL-C/VLDL was resuspended in reaction buffer and diluted to a final volume of 1:50. Serum samples were aliquoted onto a 96-well plate, incubated for $45 \mathrm{~min}$, and measured at excitation of $570 \mathrm{~nm}$ and emission at $590 \mathrm{~nm}$ using a BioTek Epoch microplate spectrophotometer.

Serum triglycerides were determined by commercially available colorimetric assay (Cayman Chemical, Ann Arbor, MI, USA). Briefly, $10 \mu \mathrm{L}$ of serum was aliquoted onto a 96-well plate and reaction was initiated with $150 \mu \mathrm{L}$ of diluted enzyme mixture solution. The plate was incubated at room temperature for 15 min and measured at a wavelength of $540 \mathrm{~nm}$ using a BioTek Epoch microplate spectrophotometer. All of the samples were performed in duplicate. The intra-assay coefficient of variation was $15.6 \%$.

\section{Serum total bile acid concentration}

Serum total bile acid content was determined using a commercially available bile acid colorimetric assay kit (Crystal Chem Inc., Elk Grove, IL). Briefly, $20 \mu \mathrm{l}$ of serum and $150 \mu \mathrm{l}$ of standard reagent were added to a 96 -well plate and incubated for $5 \mathrm{~min}$ at $37^{\circ} \mathrm{C}$. Absorbance was measured at $540 \mathrm{~nm}$ on a BioTek Epoch microplate spectrophotometer. A second standard reagent $(30 \mu \mathrm{l})$ was then added to all wells followed by a 5 min incubation at $37^{\circ} \mathrm{C}$ and absorbance read again at $540 \mathrm{~nm}$. Differences between absorbance were measured to determine total serum bile acid concentration. All samples were performed in duplicate. The intra-assay coefficient of variation was $38.5 \%$.

\section{Statistics}

Results are expressed as mean \pm standard error of the mean (SEM). Gene expression was determined as a function of $m R N A$ abundance $(A)$, where $A=1 /$ (gene of interest primer efficiency $\times \Delta \mathrm{CT}$ (g.o.i.) -average housekeeping primer efficiency $\times \Delta \mathrm{CT}$ (h.k.), where the product of efficiency and average of expression of $\beta$-actin was averaged with the product of 
efficiency and average of expression of GAPDH to determine the overall expression of the two housekeeping genes [25]. For gene expression data, each treatment group was log-transformed prior to statistical analysis.

Data was analyzed for normal distribution and homogeneity of variance prior to conducting a one-way analysis of variance (ANOVA) to determine the differences among dietary groups. Post hoc multiple comparison tests were performed on parametric data using Tukey's test with differences considered to be significant at $p=0.05$ and a tendency at $p=0.08$. Histological scoring was analyzed using the chi-square test. All statistical analyses were performed using JMP 12.2 statistical software package (SAS Institute, Cary, NC, USA).

\subsection{Results}

Fatty acid composition of diets

As shown in Table 1, dietary fat content was higher in Western diets than standard AIN diets. Fatty acid analysis (Table 3) showed Western/AP diet had the highest palmitic acid (16:0). Both Western diets contained significantly higher palmitic acid, stearic acid (18:0), palmitoleic acid (16:1n 7), and oleic acid (18:1n 9) compared to AIN diets. Essential fatty acids, linoleic acid $(18: 2 n-6)$ and $\alpha$-linoleic acid (18:3n-3) content were approximately seven-fold lower $(p<0.0001)$ in Western diets compared to AIN diets. Arachidonic acid was higher $(p<0.0001)$ in Western diets compared to AIN diets which contained negligible amounts.

Food intake, body weight and tissue weights

Figure 1 shows there was no significant differences in body weight among diet groups over 8 weeks. Shown in Table 4 , there was a tendency $(p=0.08)$ for higher final body weights for rats fed Western diets compared to AIN diets. Growing female rat fed Western diets consumed significantly more carbohydrates, fat, and total calories than rats fed standard rodent AIN diets. There were no statistically significant differences in amount of carbohydrates, fat, and total calories consumed by rats fed Western diet compared to Western/AP diet. There was a 
tendency $(p=0.08)$ for higher initial wet and dry fecal weights in rats fed Western compared to AIN diets, but no significant differences in final fecal output among diet groups. There were no statistical differences observed in feed efficiency ratio among diet groups. Rats consuming Western diets had increased ( $p<0.001)$ gonadal fat pad weights compared to rats fed AIN diets. There were no statistically significant differences in gonadal fat pad weight in rats fed Western diet compared to Western/AP diet. There were no statistically significant differences in absolute or relative liver weights among diet groups.

\section{Liver total lipid and triglyceride content}

Total lipids and triglyceride content in the liver were within the value range reported in previous studies of NAFLD [169]. There were no statistically significant differences in hepatic total lipid content among diet groups (Figure 2A). Rats fed Western diet had the highest ( $p=0.01)$ hepatic triglyceride content. Rats fed Western/AP diet showed no significant differences in liver triglyceride content compared to rats fed AIN diets (Figure 2B).

\section{Liver Histology}

Based on liver histology $14 \%$ of rats fed AIN diet had fat vacuoles in $<33 \%$ of hepatocytes (Figure 3 panel A) and $43 \%$ of rats fed AIN/AP diet had fat vacuoles in $<33 \%$ of hepatocytes (Figure 3, panel B). Higher hepatic fat infiltration was indicated by $25 \%$ of rats fed Western diet having fat vacuoles in $33-66 \%$ hepatocytes (Figure 3, panel C) while $13 \%$ of rats fed Western/AP diet had fat vacuoles in 33-66\% of hepatocytes (Figure 3, panel D). There was an overall significant $(p<0.0001)$ difference among histology scores.

\section{Liver fatty acid composition}

As shown in Table 5, rats fed Western diet had significantly higher hepatic palmitic acid content than rats fed AIN diets. While Western/AP diet contained the highest amount of palmitic 
acid, liver content in rats fed Western/AP diet was only significantly higher compared to rats fed AIN/AP diet. Western diets contained higher amounts of stearic acid and showed a tendency $(p=0.08)$ for higher hepatic stearic acid content than rats fed AIN diets. Both Western diets also contained higher amounts of palmitoleic and oleic acid than AIN diets. However, rats consuming Western diet had the highest hepatic palmitoleic acid content $(p=0.05)$. Rats fed Western diet, but not Western/AP diet had higher hepatic oleic acid content $(p=0.0005)$ compared to rats fed AIN diets. Rats consuming Western diets had significantly lower $(p<0.0001)$ hepatic linoleic and $\alpha$-linolenic acid content compared to rats consuming AIN diets, however no difference in hepatic arachidonic acid content was observed among all groups despite negligible amounts in the AIN diets.

Hepatic lipogenic gene expression

As shown in Table 6, hepatic DGAT2 gene expression was up-regulated $(p<0.01)$ in rats consuming Western diet compared to all diet groups. Western/AP diet reverted hepatic DGAT2 gene expression to that found in rats fed AIN diets. There were no statistically significant differences in hepatic gene expression of ChREBP, SREBP-1c, SREBP-2, SCD-1, FAS, PPARa, PPARY, HSL or MTTP among diet groups.

Serum liver enzymes, cholesterol, triglyceride, and bile acid measurements

As shown in Table 7 there were no statistical significant differences in serum AST, ALT, AST:ALT ratio among diet groups. Serum triglycerides, VLDL/LDL-C, HDL-C, and total cholesterol were not significantly different among diet groups. Serum bile acid concentration was significantly higher in rats fed Western diet, but not Western/AP compared to rats fed AIN diets. 


\subsection{Discussion}

Rats that were provided Western diets ingested more $(p<0.0001)$ calories than rats provided standard AIN rodent diets and had greater $(p<0.0001)$ gonadal fat pad weight, although not heavier body weight. In addition to being associated with obesity, diets that are high in saturated fats and simple carbohydrates typified by Western diets have been associated with the development of NAFLD [26,27]. Liver steatosis has also been diagnosed in non-obese patients [8]. Replacing sugars in the diet, as well as increasing fruit consumption, is recommended [28]. However, studies have reported high fructose consumption induces NAFLD by stimulating hepatic DNL $[17,26]$. Among fruits popularly consumed in the USA, apples are considered to be particularly concentrated in fructose [2,29]. In the present study, nutrient analysis of apple pomace showed the major sugar was fructose $(>30 \%)$.

Histological evaluation of liver tissue showed low fat infiltration (fat vacuoles in $<33 \%$ of hepatocytes), with no significant increase in total lipid or triglyceride content in rats consuming AIN/AP as compared to AIN diet. Hepatic gene expression of DNL transcription factors and enzymes were not upregulated and there were no increases in end products: palmitic, stearic, palmitoleic, or oleic acid content in the liver of rats fed AIN/AP when compared to the AIN diet. Furthermore, there were no significant differences in serum ALT, AST, and ALT/AST ratio to indicate liver damage and dysfunction. Conversely, rats fed Western diets showed greater hepatic lipid accumulation, as indicated by fat vacuoles in $33-66 \%$ of hepatocytes. Rats fed Western diet showed $25 \%$ when compared to $14 \%$ of animals fed Western/AP having fat vacuoles in 33-66\% of hepatocytes. Rats fed Western/AP had decreased $(p=0.04)$ hepatic triglyceride content as compared to rats fed Western diet, suggesting that substituting calories in the Western diet with $10 \%$ apple pomace attenuates hepatic triglyceride deposition.

Hepatic DNL is stimulated by FAS catalyzing synthesis of SFAs (i.e., palmitic and stearic acid). Our results showed no dietary effects on hepatic FAS gene expression. Yet, rats that were fed Western diet had higher $(p=0.0007)$ hepatic palmitic acid content when compared to 
rats fed either AIN diets. This may be explained by higher palmitic content of Western diets. Replacement with apple pomace in the Western/AP diet resulted in similar hepatic palmitic acid content to rats fed the AIN diet, but was higher than rats fed AIN/AP diet. In DNL, palmitic acid and stearic acid can be desaturated by the enzyme SCD-1 to MUFAs and palmitoleic and oleic acid, respectively. Despite no significant differences in dietary MUFA content between the Western and Western/AP diets, rats consuming Western diet had the highest $(p=0.05)$ liver palmitoleic acid content. Serum palmitoleic acid has been shown to be to be elevated in patients with liver disease and high serum VLDL [30,31]. Additionally, rats consuming a Western diet, but not Western/AP diet, had higher $(p=0.0005)$ hepatic oleic acid content as compared to rats consuming AIN diets. Studies have suggested that high oleic acid stimulates hepatic fat deposition, since oleic acid is the preferred substrate for hepatic triglyceride synthesis [32]. In a human clinical study, higher serum oleic acid was positively correlated to NAFLD [33]. In the present study, despite differences in liver MUFA content, gene expression of SCD-1 was not significantly different among diet groups. Differences in hepatic fat infiltration and fatty acid composition in the absence of changes in DNL gene expression may be due instead to diet influencing genes regulating lipolysis.

HSL catalyzes the conversion of diacylglycerols to monoacylglycerols in lipolysis [31]. PPARa and PPARy have been suggested as a potential therapeutic target for NAFLD, as the upregulation of these transcription factors results in increased use of lipids for metabolism [34,35]. Therefore, gene expression of HSL, as well as transcription factors PPAR a and PPARY, were determined to assess whether increased lipolysis was responsible for the observed hepatoprotective effects of apple pomace. In the current study, the expression of genes regulating lipolysis were not significantly different among diet groups. Besides imbalanced DNL and lipolysis, altered hepatic lipid storage and transport has been suggested to be key to NAFLD $[31,36]$. Increased gene expression of DGAT2 has been reported to promote hepatic steatosis $[37,38]$. Reducing DGAT2 has also been identified as a therapeutic target for NAFLD 
[39]. In the current study, hepatic DGAT2 gene expression was upregulated $(p=0.002)$ nearly three-fold in rats that were fed a Western diet. The mechanism for higher triglyceride content in the liver of rats fed Western diet might be explained by the combination of upregulation of DGAT2 gene expression and increased MUFAs and palmitoleic and oleic acid content in the liver, since MUFAs have been shown to be preferentially used for triglyceride synthesis [32,40]. On the other hand, polyunsaturated fatty acids (PUFAs) have been reported to have antisteatosis effects [33]. In our study, rats fed both Western diets had significantly lower hepatic PUFA (e.g., linoleic acid and a-linolenic acid) content than rats that were fed AIN diets. There was no difference in hepatic PUFA content in rats fed Western diet as compared to the Western/AP diet. Based on our results, MUFAs appeared to be the bioactive fatty acids inducing NAFLD in the Western diet.

Once synthesized triglycerides enter storage or secretory pools, hepatic MTTP regulates the packaging of triglycerides into VLDLs for transport into the circulation [41-43]. In our study, hepatic gene expression of MTTP was not significantly different among the diet groups. This was indicated by no significant changes in serum triglycerides and LDL-C/VLDL among diet groups. Overexpression of DGAT2 in mouse liver has been shown to increase liver triglyceride content, but not VLDL secretion [44]. Based on the results, greater total triglyceride accumulation in the liver of rats that were fed Western diet was due to increased triglyceride synthesis without a concomitant increase in the transport of triglycerides out of the liver. Accumulation of hepatic triglycerides without increasing circulating VLDLs may be due to the physical limitations of liver to export triglyceride-rich VLDL particles that exceed the diameter of the sinusoidal endothelia pores [45].

On the other hand, DGAT2 gene expression in the liver was not significantly upregulated in rats fed Western/AP diet, suggesting a potential therapeutic role of apple pomace in ameliorating Western diet induced NAFLD. Studies have suggested that bile represses hepatic triglyceride secretion, therefore reducing triglyceride accumulation [46]. Individuals with NAFLD 
have been reported to have higher serum bile acid [47]. Dietary fiber has been reported to decrease serum bile acids [48]. Individuals with NAFLD have been shown to consume less dietary fiber than healthy individuals [49]. Apple pomace contains a substantial amount of nondigestible carbohydrates, including: dietary fibers, pectin, and oligosaccharides [2]. In our study, serum bile acid concentration was higher in rats fed Western diet $(p<0.05)$, but not Western/AP diet as compared to rats fed AIN diets. Western diet was adjusted to remain isocaloric after substitution with apple pomace. All diets were adjusted to have $5 \%$ total fiber. No significant differences in body weight, fecal weights, and feed efficiency ratio suggested no differences in digestible energy among diet. However, differences in fiber type may be a potential mechanism. Studies have reported that dietary oligofructose decreases intra-hepatic triglycerides [50]. Hepatocytes that were isolated from non-digestible carbohydrate oligofructose-fed rats showed a reduced capacity to esterify palmitic acid [51]. In our study, rats fed Western diet, but not Western/AP, had higher ( $p=0.0007)$ palmitic acid content in the liver when compared to rats fed the AIN diet. Consumption of fruits and dietary fiber have been shown to improve liver steatosis $[49,52]$. Therefore, as a fruit-based product that is high in dietary fiber, apple pomace could potentially improve dietary fiber consumption in individuals with hepatic steatosis and the risk of NAFLD.

A study of fiber-rich colloids that were isolated from apple pomace reported increased fecal excretion of bile acids with dietary fiber by fiber acting as a bile sequestrant to improve serum lipoproteins [53]. Another potential mechanism is the effect of soluble fibers on microbiota. Mice fed a $30 \%$ fat diet supplemented with $4 \%$ pectin modulated microbiota and increased short-chain fatty acid (SCFA) production resulting in a reduction in NAFLD [54]. However, extraction and purification of isolated ingredients from food can be technologically challenging and costly. Also, nutrients in foods often act synergistically. Young male rats that were fed a standard diet supplemented with 5 or $15 \%$ apple pomace for four weeks increased cecal SCFAs $[55,56]$. However, there has been a dearth of studies investigating the effects of 
apple pomace on lipid metabolism. Bobek, et al., focusing specifically on cholesterol metabolism in the liver, reported the beneficial effects of a $5 \%(\mathrm{w} / \mathrm{w})$ apple pomace supplementation [57]. Another study reported diet-induced obese male Sprague-Dawley rats fed a high-fat diet (15\% $\mathrm{w} / \mathrm{w})$ that was substituted with $10 \%(\mathrm{w} / \mathrm{w})$ apple pomace for five weeks as compared to rats fed a high-fat diet resulted in significantly reduced liver triglyceride content, serum total triglycerides, total cholesterol, and LDL-C due to higher fecal triglyceride and cholesterol excretion [5]. In the present study, healthy growing female Sprague-Dawley rats fed the Western/AP diet attenuated hepatic fat infiltration and also attenuated elevated MUFA and triglyceride content induced by the Western diet. Additionally, elevated circulating bile acids was attenuated by apple pomace consumption. In contrast to the study on diet-induced obese male rats fed apple pomace, our study using female rats showed no improvement in serum lipoproteins [5]. Studies have shown that various types of diets that are used for developing NAFLD in experimental animals produce different effects [58]. In our study, Western diet induced hepatic steatosis was due to the dysregulation of hepatic triglyceride synthesis without changes in circulating lipoproteins. Similar hepatic effects were observed in other studies where the DGAT2 gene was overexpressed [44].

In summary, substituting calories with $10 \%$ apple pomace, despite added dietary fructose, did not promote liver steatosis in rats that were fed a standard AIN diet. Caloric substitution with fiber-rich apple pomace attenuated hepatic steatosis due to elevated hepatic MUFA content, higher circulating bile acids, and upregulated hepatic DGAT2 gene expression induced by a Western (high fat/high sugar) diet. Using a rat model, apple pomace consumption attenuated liver steatosis and had no detrimental effects on liver health. The abundance of apple pomace, currently a food processing waste by-product, has the potential to be repurposed into a sustainable food product with beneficial health properties. Further mechanistic studies, preclinical, and human clinical research investigating apple pomace for human consumption and health can offer an environmental and economical solution for fruit waste that is generated by the industrial processing of apples. 
Author Contributions: Conceptualization, RCS, VAB, and JCT; Methodology, RCS, DCW, and SNL; Software, RCS and SNL; Validation, RCS, DCW, SNL, VAB, and JCT; Formal Analysis, RCS, DCW, SNL, VAB, and JCT; Investigation, RCS and JCT; Resources, VAB and JCT; Data Curation: RCS, DCW, VAB, and JCT; Writing-Original Draft Preparation, RCS and JCT; WritingReview and Editing, RCS, DCW, SNL, VAB, and JCT; Visualization: RCS and DCW;

Supervision, RCS, DCW, VAB, and JCT; Project Administration; RCS, VAB, and JCT; Funding Acquisition; JCT.

Funding: This research was funded by Hatch WVA 1017641 and the Davis College Dean's discretionary fund.

Acknowledgements: The authors would like to thank Swilled Dog Hard Cider Company (Franklin, WV) for the donation of the apple pomace used in this project.

Conflict of Interest: The authors declare no conflict of interest. 


\subsection{References}

1. U.S. Apple Association. Apple Industry Statistics. http://usapple.org/all-aboutapples/apple-industry-statistics/. Published 2017. Accessed January 4, 2018.

2. Bhushan S, Kalia K, Sharma M, Singh B, Ahuja PS. Processing of apple pomace for bioactive molecules. Crit Rev Biotechnol. 2008;28(4):285-296.

doi:10.1080/07388550802368895

3. Shalini R, Gupta DK. Utilization of pomace from apple processing industries: a review. J Food Sci Technol. 2010;47(4):365-371. doi:10.1007/s13197-010-0061-x

4. Kaushal N, Joshi V, Vaidya D. Effect of stage of apple pomace collection and the treatment on the physico-chemical and sensory qualities of pomace Papad (fruit cloth). Indian Food Pack. 2001;55(5):64-69. http://jglobal.jst.go.jp/en/public/20090422/200902173246576860. Accessed January 10, 2018.

5. Cho KD, Han CK, Lee BH. Loss of body weight and fat and improved lipid profiles in obese rats Fed apple pomace or apple juice concentrate. J Med Food. 2013;16(9):823-830. doi:10.1089/jmf.2013.2784

6. Lonardo A, Ballestri S, Marchesini G, Angulo P, Loria P. Nonalcoholic fatty liver disease: A precursor of the metabolic syndrome. Dig Liver Dis. 2015;47(3):181-190. doi:10.1016/J.DLD.2014.09.020

7. Anderson EL, Howe LD, Jones HE, Higgins JPT, Lawlor DA, Fraser A. The prevalence of non-alcoholic fatty liver disease in children and adolescents: a systematic review and metaanalysis. Wong V, ed. PLoS One. 2015;10(10):e0140908. doi:10.1371/journal.pone.0140908 
8. Margariti E, Deutsch M, Manolakopoulos S, Papatheodoridis G V. Non-alcoholic fatty liver disease may develop in individuals with normal body mass index. Ann Gastroenterol. 2012;25(1):45-51. http://www.ncbi.nlm.nih.gov/pubmed/24713801. Accessed October 30, 2018.

9. Ishimoto T, Lanaspa MA, Rivard CJ, et al. High-fat and high-sucrose (western) diet induces steatohepatitis that is dependent on fructokinase. Hepatology. 2013;58(5):1632-1643. doi:10.1002/hep.26594

10. Mells JE, Fu PP, Sharma S, et al. Glp-1 analog, liraglutide, ameliorates hepatic steatosis and cardiac hypertrophy in C57BL/6J mice fed a Western diet. Am J Physiol Liver Physiol. 2012;302(2):G225-G235. doi:10.1152/ajpgi.00274.2011

11. Strable MS, Ntambi JM. Genetic control of de novo lipogenesis: role in diet-induced obesity. Crit Rev Biochem Mol Biol. 2010;45(3):199-214. doi:10.3109/10409231003667500

12. Wang Z, Yao T, Song Z. Involvement and mechanism of DGAT2 upregulation in the pathogenesis of alcoholic fatty liver disease. J Lipid Res. 2010;51(11):3158-3165. doi:10.1194/jr.M007948

13. Munteanu MA, Nagy GA, Mircea PA. Current management of NAFLD. Clujul Med. 2016;89(1):19-23. doi:10.15386/cjmed-539

14. Schulze C, Bangert A, Kottra G, et al. Inhibition of the intestinal sodium-coupled glucose transporter 1 (SGLT1) by extracts and polyphenols from apple reduces postprandial blood glucose levels in mice and humans. Mol Nutr Food Res. 2014;58(9):1795-1808. doi:10.1002/mnfr.201400016

15. Leontowicz M, Gorinstein S, Bartnikowska E, Leontowicz H, Kulasek G, Trakhtenberg S. Sugar beet pulp and apple pomace dietary fibers improve lipid metabolism in rats fed cholesterol. Food Chem. 2001;72(1):73-78. doi:10.1016/S0308-8146(00)00207-7 
16. Lim JS, Mietus-Snyder M, Valente A, Schwarz J-M, Lustig RH. The role of fructose in the pathogenesis of NAFLD and the metabolic syndrome. Nat Rev Gastroenterol Hepatol. 2010;7(5):251-264. doi:10.1038/nrgastro.2010.41

17. Mock K, Lateef S, Benedito VA, Tou JC. High-fructose corn syrup-55 consumption alters hepatic lipid metabolism and promotes triglyceride accumulation. J Nutr Biochem. 2017;39:3239.

18. Rinella ME, Sanyal AJ. Management of NAFLD: a stage-based approach. Nat Rev Gastroenterol Hepatol. 2016;13(4):196-205. doi:10.1038/nrgastro.2016.3

19. Vilà L, Roglans N, Perna V, et al. Liver AMP/ATP ratio and fructokinase expression are related to gender differences in AMPK activity and glucose intolerance in rats ingesting liquid fructose. J Nutr Biochem. 2011;22(8):741-751. doi:10.1016/J.JNUTBIO.2010.06.005

20. National Research Council. Guide for the Care and Use of Laboratory Animals: Eighth Edition - National Research Council, Division on Earth and Life Studies, Institute for Laboratory Animal Research, Committee for the Update of the Guide for the Care and Use of Laboratory Animals - Google Books. 8th ed. Washington D.C.; 2010.

https://books.google.com/books?hl=en\&lr=\&id=Vp5mgXtxYdQC\&oi=fnd\&pg=PP2\&dq=national+ research+council+2010+rats\&ots=FrTgd1JCI5\&sig=eZ_vGWk36QfLScXnMbS_BgT8XU\#v=on epage\&q=national research council 2010 rats\&f=false. Accessed February 27, 2018.

21. Blainski A, Lopes G, de Mello J, Blainski A, Lopes GC, de Mello JCP. Application and Analysis of the Folin Ciocalteu Method for the Determination of the Total Phenolic Content from Limonium Brasiliense L. Molecules. 2013;18(6):6852-6865. doi:10.3390/molecules18066852

22. Bligh EG, Dyer WJ. A rapid method of total lipid extraction and purification. Can J Biochem Physiol. 1959;37(1):911-917. doi:10.1139/059-099 
23. Fritsche KL, Johnston P V. Effect of dietary a-linolenic acid on growth, metastasis, fatty acid profile and prostaglandin production of two murine mammary adenocarcinomas. J Nutr. 1990;120(12):1601-1609. doi:10.1093/jn/120.12.1601

24. Brunt EM, Janney CG, Bisceglie AM, Neuschwander-Tetri BA, Bacon BR. Nonalcoholic steatohepatitis: a proposal for grading and staging the histological lesions. Am J Gastroenterol. 1999;94(9):2467-2474. doi:10.1111/j.1572-0241.1999.01377.x

25. Jacometo CB, Schmitt E, Pfeifer LFM, et al. Linoleic and $\alpha$-linolenic fatty acid consumption over three generations exert cumulative regulation of hepatic expression of genes related to lipid metabolism. Genes Nutr. 2014;9(4):405. doi:10.1007/s12263-014-0405-7

26. Loomba R, Sanyal AJ. The global NAFLD epidemic. Nat Rev Gastroenterol Hepatol. 2013;10(11):686-690. doi:10.1038/nrgastro.2013.171

27. Chalasani N, Younossi Z, Lavine JE, et al. The diagnosis and management of nonalcoholic fatty liver disease: Practice guidance from the American Association for the Study of Liver Diseases. Hepatology. 2018;67(1):328-357. doi:10.1002/hep.29367

28. Department of Health and Human Services. Dietary Guidelines for Americans 20152020 - U.S. Department of Health and Human Services, U.S. Department of Agriculture. New York NY: Skyhorse Publishing; 2017.

29. Southgate D, Paul AA, Dean AC, Christie AA. Free sugars in foods. Int J Food Sci Nutr. 1978;32(5):335-347. http://www.ncbi.nlm.nih.gov/pubmed/363937. Accessed January 24, 2018.

30. Nestel P, Clifton P, Noakes M. Effects of increasing dietary palmitoleic acid compared with palmitic and oleic acids on plasma lipids of hypercholesterolemic men. J Lipid Res. 1994;35(4):656-662. http://www.ncbi.nlm.nih.gov/pubmed/8006520. Accessed June 4, 2018. 
31. Lee JJ, Lambert JE, Hovhannisyan Y, et al. Palmitoleic acid is elevated in fatty liver disease and reflects hepatic lipogenesis. Am J Clin Nutr. 2015;101(1):34-43.

doi:10.3945/ajcn.114.092262

32. Gambino R, Bugianesi E, Rosso C, et al. Different Serum Free Fatty Acid Profiles in NAFLD Subjects and Healthy Controls after Oral Fat Load. Int J Mol Sci. 2016;17(4):479. doi:10.3390/ijms17040479

33. Juárez-Hernández E, Chávez-Tapia NC, Uribe M, Barbero-Becerra VJ. Role of bioactive fatty acids in nonalcoholic fatty liver disease. Nutr J. 2016;15(1):72. doi:10.1186/s12937-0160191-8

34. Kersten S, Desvergne B, Wahli W. Roles of PPARs in health and disease. Nature. 2000;405(6785):421-424. doi:10.1038/35013000

35. Fuchs CD, Claudel T, Trauner M. Role of metabolic lipases and lipolytic metabolites in the pathogenesis of NAFLD. Trends Endocrinol Metab. 2014;25(11):576-585. doi:10.1016/J.TEM.2014.08.001

36. Romeo S, Kozlitina J, Xing C, et al. Genetic variation in PNPLA3 confers susceptibility to nonalcoholic fatty liver disease. Nat Genet. 2008;40(12):1461-1465. doi:10.1038/ng.257

37. Monetti M, Levin MC, Watt MJ, et al. Dissociation of hepatic steatosis and insulin resistance in mice overexpressing DGAT in the liver. Cell Metab. 2007;6(1):69-78. doi:10.1016/J.CMET.2007.05.005

38. Yu XX, Murray SF, Pandey SK, et al. Antisense oligonucleotide reduction of DGAT2 expression improves hepatic steatosis and hyperlipidemia in obese mice. Hepatology. 2005;42(2):362-371. doi:10.1002/hep.20783 
39. Samuel VT, Shulman GI. Nonalcoholic fatty liver disease as a nexus of metabolic and hepatic diseases. Cell Metab. 2018;27(1):22-41. doi:10.1016/J.CMET.2017.08.002

40. Sampath H, Miyazaki M, Dobrzyn A, Ntambi JM. Stearoyl-CoA desaturase-1 mediates the pro-lipogenic effects of dietary saturated fat. J Biol Chem. 2007;282(4):2483-2493. doi:10.1074/jbc.M610158200

41. Lichtenstein AH, Van Horn L, Brands M, et al. Very low fat diets. Circulation. 1998;98(9):935-939. http://www.ncbi.nlm.nih.gov/pubmed/9738652. Accessed January 8, 2018.

42. Fabbrini E, Sullivan S, Klein S. Obesity and nonalcoholic fatty liver disease: Biochemical, metabolic, and clinical implications. Hepatology. 2010;51(2):679-689. doi:10.1002/hep.23280

43. Chang X, Yan H, Fei J, et al. Berberine reduces methylation of the MTTP promoter and alleviates fatty liver induced by a high-fat diet in rats. J Lipid Res. 2010;51(9):2504-2515. doi:10.1194/jr.M001958

44. Millar JS, Stone SJ, Tietge UJF, et al. Short-term overexpression of DGAT1 or DGAT2 increases hepatic triglyceride but not VLDL triglyceride or apoB production. J Lipid Res. 2006;47(10):2297-2305. doi:10.1194/Jr.M600213-JLR200

45. Olofsson S-O, Stillemark-Billton P, Asp L. Intracellular sssembly of VLDL: two major steps in separate cell compartments. Trends Cardiovasc Med. 2000;10(8):338-345. doi:10.1016/S1050-1738(01)00071-8

46. $\quad \mathrm{Li} \mathrm{T}$, Chiang JYL. Bile Acid signaling in liver metabolism and diseases. J Lipids. 2012;2012:754067. doi:10.1155/2012/754067

47. de Caestecker JS, Jazrawi RP, Nisbett JA, Joseph AE, Maxwell JD, Northfield TC. Direct assessment of the mechanism for a raised serum bile acid level in chronic liver disease. 
Eur J Gastroenterol Hepatol. 1995;7(10):955-961.

http://www.ncbi.nlm.nih.gov/pubmed/8590141. Accessed June 25, 2018.

48. Ebihara K, Schneeman BO. Interaction of bile acids, phospholipids, cholesterol and triglyceride with dietary fibers in the small intestine of rats. J Nutr. 1989;119(8):1100-1106. doi:10.1093/jn/119.8.1100

49. Zolfaghari H, Askari G, Siassi F, Feizi A, Sotoudeh G. Intake of nutrients, fiber, and sugar in patients with nonalcoholic fatty liver disease in comparison to healthy individuals. Int $J$ Prev Med. 2016;7:98. doi:10.4103/2008-7802.188083

50. Busserolles J, Gueux E, Rock E, Demigné C, Mazur A, Rayssiguier Y. Oligofructose protects against the hypertriglyceridemic and pro-oxidative effects of a high fructose diet in rats. J Nutr. 2003;133(6):1903-1908. doi:10.1093/jn/133.6.1903

51. Kok N, Roberfroid M, Robert A, Delzenne N. Involvement of lipogenesis in the lower VLDL secretion induced by oligofructose in rats. $\mathrm{Br} J$ Nutr. 1996;76(6):881-890. doi:10.1079/BJN19960094

52. Farrell GC, Larter CZ. Nonalcoholic fatty liver disease: From steatosis to cirrhosis. Hepatology. 2006;43(S1):S99-S112. doi:10.1002/hep.20973

53. Sembries S, Dongowski G, Mehrländer K, Will F, Dietrich $\mathrm{H}$. Dietary fiber-rich colloids from apple pomace extraction juices do not affect food intake and blood serum lipid levels, but enhance fecal excretion of steroids in rats. J Nutr Biochem. 2004;15(5):296-302. doi:10.1016/J.JNUTBIO.2003.12.005

54. Li W, Zhang K, Yang H. Pectin Alleviates High Fat (Lard) Diet-Induced Nonalcoholic Fatty Liver Disease in Mice: Possible Role of Short-Chain Fatty Acids and Gut Microbiota 
Regulated by Pectin. J Agric Food Chem. 2018;66(30):8015-8025.

doi:10.1021/acs.jafc.8b02979

55. Kosmala M, Kołodziejczyk K, Zduńczyk Z, Juśkiewicz J, Boros D. Chemical composition of natural and polyphenol-free apple pomace and the effect of this dietary ingredient on intestinal fermentation and serum lipid parameters in rats. J Agric Food Chem. 2011;59(17):9177-9185. doi:10.1021/jf201950y

56. Juśkiewicz J, Żary-Sikorska E, Zduńczyk Z, Król B, Jarosławska J, Jurgoński A. Effect of dietary supplementation with unprocessed and ethanol-extracted apple pomaces on caecal fermentation, antioxidant and blood biomarkers in rats. Br J Nutr. 2012;107(8):1138-1146. doi:10.1017/S0007114511004144

57. Bobek P, Ozdín L, Hromadová M. The effect of dried tomato, grape and apple pomace on the cholesterol metabolism and antioxidative enzymatic system in rats with hypercholesterolemia. Mol Nutr Food Res. 1998;42(5):317-320. doi:10.1002/(SICI)15213803(199810)42:05<317::AID-FOOD317>3.0.CO;2-Y

58. Shojaei Zarghani S, Soraya H, Zarei L, Alizadeh M. Comparison of three different dietinduced non alcoholic fatty liver disease protocols in rats: a pilot study. Pharm Sci. 2016;22(1):9-15. doi:10.15171/PS.2016.03 
Table 1. Composition of rodent diets substituted with apple pomace $(10 \% \mathrm{~g} / \mathrm{kg})$ fed to growing female rats.

\begin{tabular}{ccccc}
\hline \multicolumn{5}{c}{ Diet Groups ${ }^{1}$} \\
& AIN & AIN/AP & Western & Western/AP \\
\hline Ingredients (g/kg) & & & & \\
Apple pomace & 0.0 & 100.0 & 0.0 & 100.0 \\
Corn Starch & 397.486 & 392.086 & 63.36 & 57.96 \\
Maltodextrin & 132.0 & 132.0 & 60.0 & 60.0 \\
Sucrose & 100.0 & 43.9 & 340.0 & 283.9 \\
Total Dietary Fiber & 50.0 & 50.0 & 50.0 & 50.0 \\
Insoluble Fiber ${ }^{2}$ & 50.0 & 39.0 & 50.0 & 39.0 \\
Soluble Fiber ${ }^{3}$ & 0.0 & 11.0 & 0.0 & 11.0 \\
Anhydrous Milkfat & 0.0 & 0.0 & 210.0 & 210.0 \\
Soybean Oil & 70.0 & 68.7 & 20.0 & 18.7 \\
Casein & 200.0 & 196.0 & 195.0 & 191.0 \\
L-Cystine & 3.0 & 3.0 & 3.0 & 3.0 \\
Vitamin Mix & 10.0 & 10.0 & 12.5 & 12.5 \\
Mineral Mix & 35.0 & 35.0 & 43.0 & 43.0 \\
Choline Bitartrate & 2.5 & 2.5 & 3.1 & 3.1 \\
TBHQ, antioxidant & 0.014 & 0.014 & 0.04 & 0.04 \\
Polyphenols & 0.0015 & 0.0029 & 0.0008 & 0.0032 \\
\hline Macronutrients (\% kcal) & & & & \\
Protein & 18.8 & 18.9 & 14.8 & 14.8 \\
Fat & 17.2 & 17.3 & 44.6 & 44.8 \\
Carbohydrate & 63.9 & 63.7 & 40.6 & 40.4 \\
\hline Calories (kcal/g) & 3.8 & $\mathbf{3 . 7}$ & $\mathbf{4 . 7}$ & $\mathbf{4 . 7}$ \\
\hline
\end{tabular}

${ }^{1}$ Abbreviations: AIN, the American Institute of Nutrition; AP, apple pomace; TBHQ, tertbutylhydroquinone. ${ }^{2}$ Insoluble fiber is cellulose. ${ }^{3}$ Soluble fiber is mainly pectin [2]. 
Table 2. Composition of freeze-dried apple pomace substituted $(10 \% \mathrm{~g} / \mathrm{kg})$ into diets fed to growing female rats.

\begin{tabular}{ll}
\hline Macronutrients (\%) \\
\hline Protein & 3.56 \\
Fat & 1.3 \\
Carbohydrates & 68.1 \\
\hline Sugars (\%) & 32.5 \\
Fructose & 9.77 \\
Glucose & 13.9 \\
Sucrose & $<0.1$ \\
Maltose & $<0.1$ \\
Lactose & \\
\hline Dietary Fiber (\%) & 22.2 \\
Insoluble Dietary Fiber & 11.0 \\
\hline Soluble Dietary Fiber & 0.029 \\
\hline Polyphenols (g/kg) & 387 \\
\hline Calories (kcal/100 g) & \\
\hline
\end{tabular}


Table 3. Fatty acid analysis of rodent diets substituted with apple pomace $(10 \% \mathrm{~g} / \mathrm{kg})$.

\begin{tabular}{|c|c|c|c|c|c|}
\hline \multirow{2}{*}{ Measurements } & \multicolumn{5}{|l|}{ Treatments $^{1}$} \\
\hline & AIN & AIN/AP & Western & Western/AP & $p$-Value \\
\hline \multicolumn{6}{|l|}{ SFAs } \\
\hline Palmitic acid (16:0) & $11.36 \pm 0.09^{c}$ & $11.14 \pm 0.15^{c}$ & $32.19 \pm 0.03^{b}$ & $32.92 \pm 0.30^{a}$ & $<0.0001$ \\
\hline Stearic acid (18:0) & $3.56 \pm 0.25^{b}$ & $3.72 \pm 0.06^{b}$ & $9.94 \pm 0.11^{a}$ & $10.24 \pm 0.06^{a}$ & $<0.0001$ \\
\hline \multicolumn{6}{|l|}{ MUFAs } \\
\hline Palmitoleic acid (16:1n-7) & $0 \pm 0.00^{b}$ & $0 \pm 0.00^{b}$ & $1.44 \pm 0.01^{a}$ & $1.44 \pm 0.02^{a}$ & $<0.0001$ \\
\hline Oleic acid (18:1n-9) & $19.09 \pm 0.10^{b}$ & $18.35 \pm 0.33^{b}$ & $22.96 \pm 0.11^{a}$ & $22.95 \pm 0.17^{a}$ & $<0.0001$ \\
\hline \multicolumn{6}{|l|}{ PUFAs } \\
\hline Linoleic acid (18:2 n-6) & $50.12 \pm 0.55^{a}$ & $51.41 \pm 2.41^{a}$ & $6.99 \pm 0.09 b$ & $7.04 \pm 0.06^{b}$ & $<0.0001$ \\
\hline a-linolenic acid (18:3 n-3) & $7.08 \pm 0.13^{a}$ & $7.13 \pm 0.70^{a}$ & $1.04 \pm 0.01^{b}$ & $1.05 \pm 0.02^{b}$ & $<0.0001$ \\
\hline Arachidonic acid (20:4 n-6) & $0 \pm 0.00^{b}$ & $0 \pm 0.00^{b}$ & $0.13 \pm 0.00^{a}$ & $0.14 \pm 0.00^{a}$ & $<0.0001$ \\
\hline
\end{tabular}

Values expressed as mean \pm standard error of the mean (SEM, $n=5$ samples/group). Different superscript letters a, $b$, and $c$ within. The same row indicates significant difference at $p<0.05$ by one-way ANOVA followed by Tukey's test. Abbreviations: MUFAs, monounsaturated fatty acids; PUFAs, polyunsaturated fatty, acids; SFAs, saturated fatty acids. 
Table 4. Effect of consumption of different diets substituted with apple pomace $(10 \% \mathrm{~g} / \mathrm{kg})$ by growing female rats on caloric intake, body weight, and liver weight following eight weeks of feeding.

\begin{tabular}{|c|c|c|c|c|c|}
\hline \multirow{2}{*}{ Measurements } & \multicolumn{5}{|l|}{ Treatments $^{1}$} \\
\hline & AIN & AIN/AP & Western & Western/AP & $p$-Value \\
\hline Caloric intake (kcal) & $2946 \pm 85^{b}$ & $2757 \pm 62^{b}$ & $3373 \pm 71^{a}$ & $3443 \pm 134^{a}$ & $<0.0001$ \\
\hline $\mathrm{CHO}$ intake (kcal) & $1769 \pm 54^{a}$ & $1708 \pm 39 a$ & $1354 \pm 29 b$ & $1347 \pm 54 b$ & $<0.0001$ \\
\hline Fat intake(kcal) & $476 \pm 15^{b}$ & $464 \pm 11^{b}$ & $1487 \pm 32^{a}$ & $1494 \pm 60^{a}$ & $<0.0001$ \\
\hline Initial bwt (g) & $95 \pm 3$ & $92 \pm 3$ & $95 \pm 3$ & $95 \pm 3$ & 0.80 \\
\hline Final bwt $(g)$ & $216 \pm 4$ & $216 \pm 8$ & $228 \pm 5$ & $234 \pm 5$ & 0.08 \\
\hline Total bwt gain $(\mathrm{g})$ & $121 \pm 4$ & $124 \pm 7$ & $133 \pm 6$ & $138 \pm 6$ & 0.17 \\
\hline Wet Initial Fecal Weight (g) & $0.82 \pm 0.06^{b}$ & $0.75 \pm 0.11^{b}$ & $1.30 \pm 0.23^{a}$ & $1.14 \pm 0.13^{a, b}$ & 0.06 \\
\hline Dry Initial Fecal Weight (g) & $0.67 \pm 0.06$ & $0.54 \pm 0.14$ & $1.269 \pm 0.27$ & $0.75 \pm 0.21$ & 0.08 \\
\hline Wet Final Fecal Weight (g) & $0.30 \pm 0.18^{b}$ & $0.28 \pm 0.16^{b}$ & $0.91 \pm 0.34^{a}$ & $0.63 \pm 0.23^{a, b}$ & 0.03 \\
\hline Dry Final Fecal Weight (g) & $0.23 \pm 0.13$ & $0.19 \pm 0.10$ & $0.78 \pm 0.27$ & $0.50 \pm 0.16$ & 0.13 \\
\hline Feed Efficiency Ratio & $0.17 \pm 0.01$ & $0.17 \pm 0.01$ & $0.18 \pm 0.01$ & $0.18 \pm 0.01$ & 0.13 \\
\hline Gonadal fat pad weight (g) & $4.12 \pm 0.26^{b}$ & $3.46 \pm 0.44^{b}$ & $5.87 \pm 0.24^{a}$ & $5.96 \pm 0.23^{a}$ & $<0.0001$ \\
\hline Liver weight $(\mathrm{g})$ & $7.50 \pm 0.24$ & $7.44 \pm 0.37$ & $8.05 \pm 0.30$ & $7.98 \pm 0.24$ & 0.35 \\
\hline Relative liver weight (mg/g bwt) & $3.47 \pm 0.078$ & $3.45 \pm 0.068$ & $3.52 \pm 0.067$ & $3.41 \pm 0.048$ & 0.69 \\
\hline
\end{tabular}

${ }^{1}$ Values expressed as mean \pm SEM ( $n=6-8$ rats/group). Different superscript letters a and b within the same row. Indicate significant difference at $p<0.05$ by one-way ANOVA followed by Tukey's test. Abbreviations: Bwt, body weight; CHO, carbohydrate. 
Table 5. Effect of consumption of different diets substituted with apple pomace $(10 \% \mathrm{~g} / \mathrm{kg})$ by growing female rats on hepatic fatty acid composition following 8 weeks of feeding.

\begin{tabular}{|c|c|c|c|c|c|}
\hline \multirow{2}{*}{ Measurements (\%) } & \multicolumn{5}{|l|}{ Treatments $^{1}$} \\
\hline & AIN & AIN/AP & Western & Western/AP & $p$-Value \\
\hline \multicolumn{6}{|l|}{ SFAs } \\
\hline Palmitic acid (16:0) & $\begin{array}{l}19.10 \pm 0.57 \\
b, c\end{array}$ & $18.40 \pm 0.53^{c}$ & $21.44 \pm 0.53^{a}$ & $21.08 \pm 0.53^{a, b}$ & 0.0007 \\
\hline Stearic acid (18:0) & $14.46 \pm 0.65$ & $14.06 \pm 0.60$ & $14.83 \pm 0.83$ & $16.28 \pm 0.60$ & 0.08 \\
\hline \multicolumn{6}{|l|}{ MUFAs } \\
\hline Palmitoleic acid (16:1n-7) & $0.57 \pm 0.25^{b}$ & $0.81 \pm 0.25^{b}$ & $1.61 \pm 0.23$ & $0.76 \pm 0.25^{b}$ & 0.05 \\
\hline Oleic acid $(18: 1 n-9)$ & $11.25 \pm 1.65^{b}$ & $10.72 \pm 1.39 b$ & $19.55 \pm 1.39$ a & $15.95 \pm 1.30^{a, b}$ & 0.0005 \\
\hline \multicolumn{6}{|l|}{ PUFAs } \\
\hline Linoleic acid (18:2n-6) & $22.44 \pm 1.09$ a & $25.12 \pm 1.09$ a & $9.28 \pm 1.09^{b}$ & $8.38 \pm 1.09^{b}$ & $<0.0001$ \\
\hline$\alpha$-linoleic acid (18:3n-3) & $1.04 \pm 0.14^{\mathrm{a}}$ & $1.28 \pm 0.15^{a}$ & $0.23 \pm 0.14^{b}$ & $0.22 \pm 0.14^{b}$ & $<0.0001$ \\
\hline Arachidonic acid (20:4n-6) & $13.26 \pm 1.00$ & $11.99 \pm 1.61$ & $13.20 \pm 1.00$ & $14.56 \pm 1.00$ & 0.37 \\
\hline
\end{tabular}


${ }^{1}$ Values expressed as mean \pm SEM ( $n=6-8$ rats/group). Different superscript letters $a$, b, and $c$ within the same row indicate significant difference at $p<0.05$ by one-way ANOVA followed by Tukey's test. Abbreviations: MUFAs, monounsaturated fatty acids; PUFAs, polyunsaturated fatty acids; SFAs, saturated fatty acids. 
Table 6. Effect of consumption of different diets substituted with apple pomace $(10 \% \mathrm{~g} / \mathrm{kg})$ by growing female rats on hepatic lipid metabolism gene expression following 8 weeks of feeding.

\begin{tabular}{|c|c|c|c|c|c|}
\hline \multirow{2}{*}{ Measurements } & \multicolumn{5}{|l|}{ Treatments $^{1}$} \\
\hline & AIN & AIN/AP & Western & Western/AP & $p$-Value \\
\hline \multicolumn{6}{|l|}{ Lipogenesis } \\
\hline ChREBP & $0.074 \pm 0.004$ & $0.069 \pm 0.006$ & $0.078 \pm 0.006$ & $0.077 \pm 0.009$ & 0.76 \\
\hline SREBP-1C & $0.118 \pm 0.027$ & $0.132 \pm 0.041$ & $0.136 \pm 0.038$ & $0.117 \pm 0.031$ & 0.11 \\
\hline SREBP-2 & $0.088 \pm 0.020$ & $0.080 \pm 0.012$ & $0.083 \pm 0.017$ & $0.092 \pm 0.026$ & 0.92 \\
\hline FAS & $0.116 \pm 0.034$ & $0.096 \pm 0.015$ & $0.121 \pm 0.028$ & $0.156 \pm 0.075$ & 0.15 \\
\hline SCD-1 & $0.234 \pm 0.084$ & $0.216 \pm 0.036$ & $0.223 \pm 0.073$ & $0.309 \pm 0.080$ & 0.13 \\
\hline \multicolumn{6}{|l|}{ Lipolysis } \\
\hline PPARa & $0.088 \pm 0.016$ & $0.099 \pm 0.013$ & $0.098 \pm 0.027$ & $0.076 \pm 0.012$ & 0.74 \\
\hline PPARY & $0.054 \pm 0.021$ & $0.053 \pm 0.013$ & $0.053 \pm 0.016$ & $0.052 \pm 0.026$ & 0.99 \\
\hline HSL & $0.085 \pm 0.019$ & $0.087 \pm 0.011$ & $0.084 \pm 0.023$ & $0.074 \pm 0.027$ & 0.89 \\
\hline \multicolumn{6}{|c|}{ Storage and Transport } \\
\hline DGAT2 & $0.171 \pm 0.020^{b}$ & $0.151 \pm 0.025^{b}$ & $0.617 \pm 0.161^{a}$ & $0.213 \pm 0.039 b$ & 0.002 \\
\hline MTTP & $0.145 \pm 0.026$ & $0.090 \pm 0.008$ & $0.164 \pm 0.044$ & $0.151 \pm 0.035$ & 0.43 \\
\hline
\end{tabular}

${ }^{1}$ Values expressed as mean \pm SEM ( $n=6-8$ rats/group) of transcript abundance (A) of gene of interest relative to housekeeping genes $\beta$-actin and GAPDH. a Different superscript letters a and b within the same row indicate significant difference at $p<0.05$ by one-way ANOVA followed by Tukey's test. Abbreviations: ChREBP, carbohydrate element response binding protein; DGAT2,

diacylglycerol O-acyltransferase 2; FAS, fatty acid synthase; HSL, hormone sensitive lipase; MTTP, microsomal triglyceride transfer protein; PPARa, peroxisome proliferator-activated receptor alpha; PPARy, peroxisome proliferator-activated receptor gamma; SCD1, stearoyl-CoA desaturase-1; SREBP-1c, sterol regulatory binding protein-1c; SREBP-2, sterol regulatory binding protein-2. 
Table 7. Effect of consumption of different diets substituted with apple pomace $(10 \% \mathrm{~g} / \mathrm{kg})$ by growing female rats on serum measurements of liver function enzymes, cholesterol, and bile acids following 8 weeks of feeding.

\begin{tabular}{llllll}
\hline Serum Measurements & AIN & AIN/AP & Western & Western/AP & $p$-Value \\
\hline AST $(\mathrm{U} / \mathrm{L})$ & $129.48 \pm 52.86$ & $212.50 \pm 37.86$ & $283.63 \pm 45.30$ & $259.67 \pm 48.96$ & 0.69 \\
ALT $(\mathrm{U} / \mathrm{L})$ & $107.63 \pm 19.59$ & $118.71 \pm 43.60$ & $94.5 \pm 12.58$ & $133.5 \pm 30.59$ & 0.78 \\
AST:ALT ratio & $3.16 \pm 0.45$ & $2.79 \pm 0.28$ & $2.97 \pm 0.26$ & $2.84 \pm 0.17$ & 0.83 \\
VLDL/LDL-C $(\mathrm{mg} / \mathrm{dl})$ & $41.59 \pm 4.17$ & $41.74 \pm 2.38$ & $40.42 \pm 6.44$ & $41.29 \pm 6.74$ & 0.99 \\
HDL-C $(\mathrm{mg} / \mathrm{dl})$ & $17.29 \pm 2.18$ & $18.95 \pm 1.97$ & $18.21 \pm 2.13$ & $21.85 \pm 1.82$ & 0.43 \\
Total Cholesterol $(\mathrm{mg} / \mathrm{dl})$ & $58.89 \pm 3.43$ & $60.38 \pm 3.38$ & $59.80 \pm 5.69$ & $57.98 \pm 7.85$ & 0.99 \\
Triglyceride $(\mathrm{mg} / \mathrm{dl})$ & $55.81 \pm 8.17$ & $47.39 \pm 9.04$ & $58.64 \pm 9.38$ & $50.04 \pm 8.81$ & 0.84 \\
Total Bile Acids $(\mu \mathrm{mol} / \mathrm{L})$ & $30.00 \pm 3.13^{\mathrm{b}}$ & $28.80 \pm 7.69 \mathrm{~b}$ & $54.13 \pm 7.966^{\mathrm{a}}$ & $31.52 \pm 3.69 \mathrm{a}$ ab & 0.02
\end{tabular}

${ }^{1}$ Values expressed as mean \pm SEM of $n=6-8$ rats/group. Different superscript letters a and b within the same row indicate significant difference at $p<0.05$ by one-way ANOVA followed by Tukey's test. Abbreviations: AST, aspartate aminotransferase; ALT, alanine aminotransferase; VLDL, very-low density lipoprotein, LDL-C, low density lipoprotein-cholesterol; HDL-C, high density lipoprotein-cholesterol. 


\section{Figure Legend}

Figure 1. Growth curve of growing female rats consuming different diets including apple pomace over 8 weeks.

Figure 2. (A) Total hepatic lipid percentage of rodents consuming different diets including apple pomace. (B) Total hepatic triglyceride content of rodents consuming different diets including apple pomace. Different letters $a$ and $b$ indicate significant difference $a t p<0.05$ by one-way ANOVA followed by Tukey's test

Figure 3. Representative histological staining images of livers of growing female rats consuming different diets including apple pomace. 


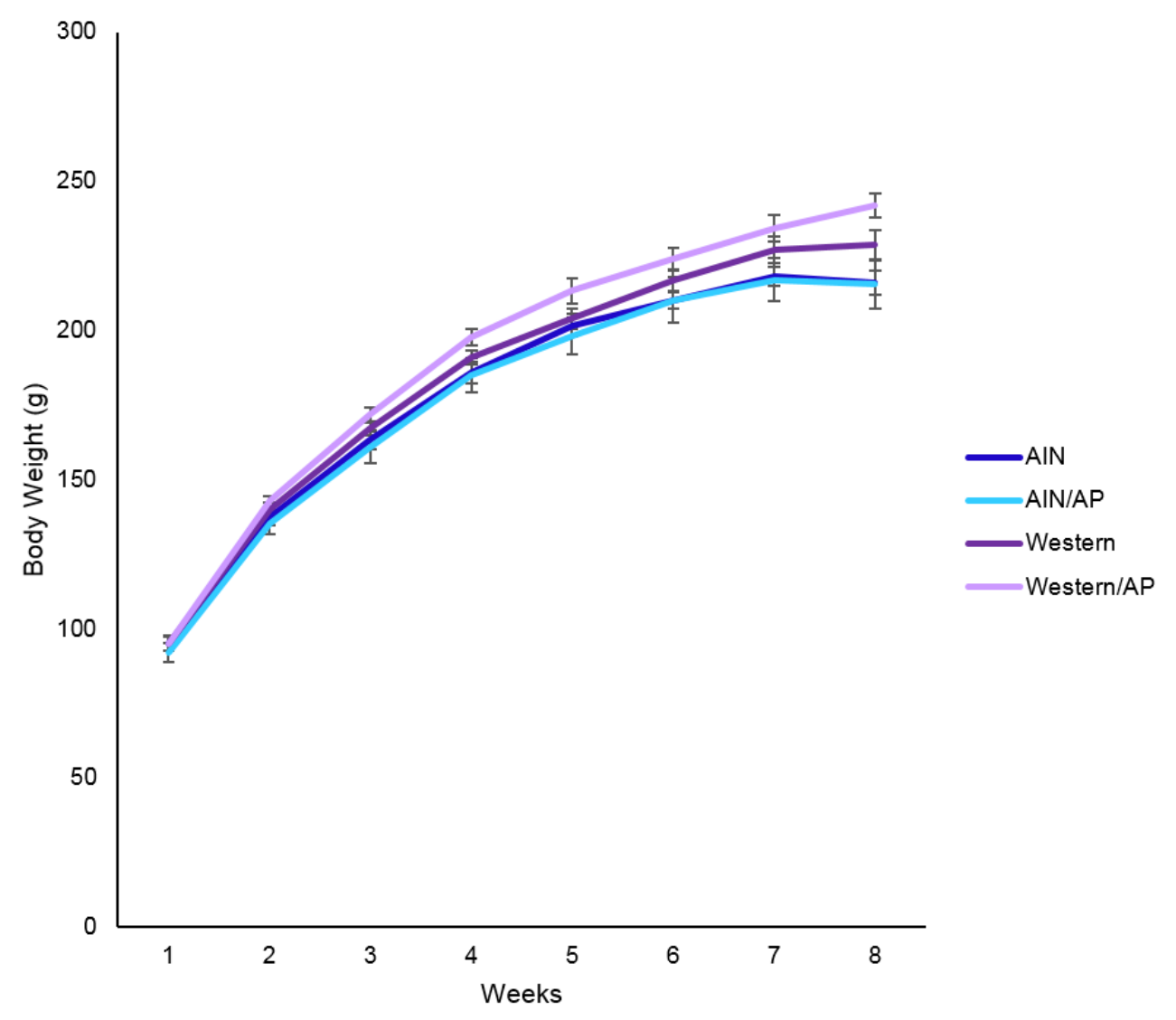

Figure 1. Growth curve of rats fed different diets containing apple pomace. 

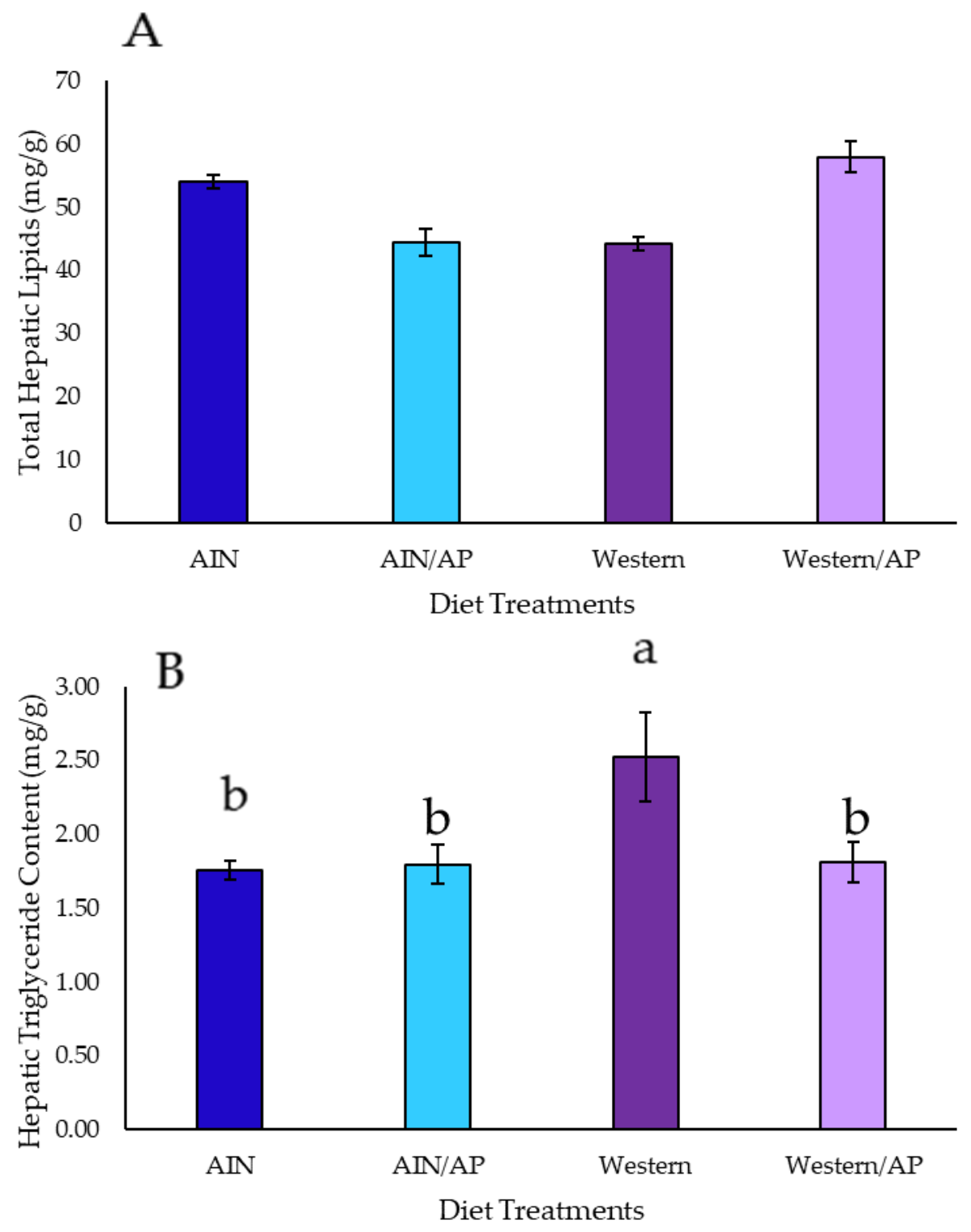

Figure 2. (A) Total hepatic lipid content $(\mathrm{mg} / \mathrm{g})$ and $($ B) Total hepatic triglyceride content $(\mathrm{mg} / \mathrm{g})$ of growing female rats consuming different diets substituted with apple pomace $(10 \% \mathrm{w} / \mathrm{w})$ following eight weeks of feeding. Different letters $a$ and $b$ indicate significant difference at $p<$ 0.05 by one-way ANOVA followed by Tukey's test. 


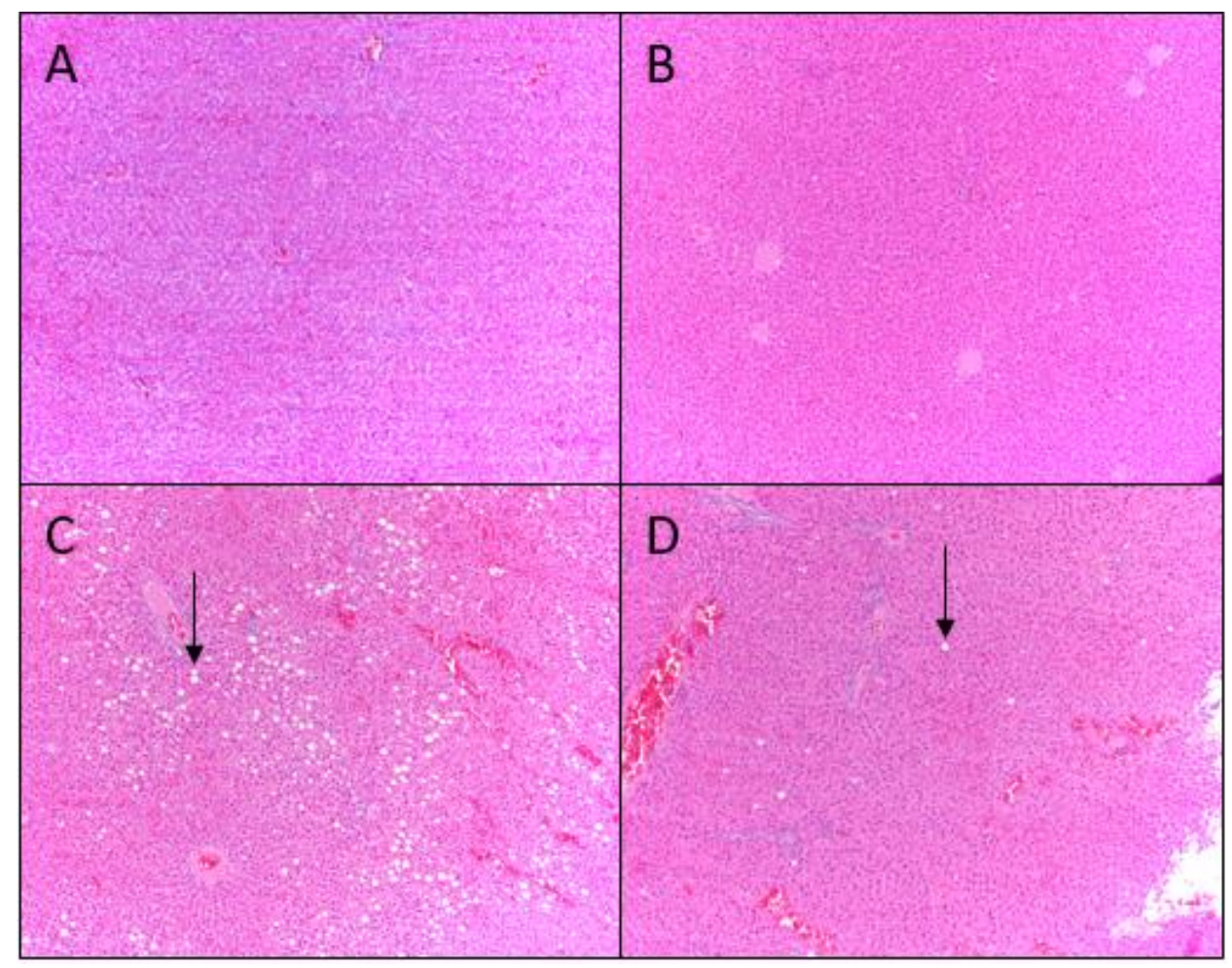

Histological steatosis scores for diet groups.

\begin{tabular}{lcllll}
\hline Measurement & AIN & AIN/AP & Western & Western/AP & $\boldsymbol{p}$-Value \\
\hline Steatosis Grade & & & & & $<0.0001$ \\
0 & 6 & 4 & - & - & \\
1 & 1 & 3 & 6 & 7 & \\
2 & - & - & 2 & 1 & \\
3 & - & - & - & - &
\end{tabular}

Figure 3. Representative histological staining images of livers of growing female rats consuming (A) AIN, (B) AIN/AP, (C) Western, or (D) Western/AP following eight weeks of feeding. Arrow indicates fat deposition. Scores analyzed by chi-square test at $p<0.05$. 


\subsection{Chapter 2}

Apple pomace attenuates liver-adipose crosstalk and improves antioxidant status in young female rats consuming a Western diet

R. Chris Skinner ${ }^{1}$, Derek C. Warren ${ }^{1}$, Minahal Naveed ${ }^{1}$, Garima Agarwal ${ }^{1}$, Vagner A. Benedito², Janet C. Tou ${ }^{1}$

${ }^{1}$ Division of Animal and Nutritional Sciences, ${ }^{2}$ Division of Plant and Soil Sciences, West Virginia University, Morgantown, WV 26506

Corresponding Author:

Janet C. Tou, PhD

Division of Animal and Nutritional Sciences

West Virginia University

Morgantown, WV 26506

Tel: (304)293-1919

Fax: (304)293-2232

e-mail: janet.tou@mail.wvu.edu 


\subsection{Abstract}

Non-alcoholic fatty liver disease, the most prevalent liver condition, can progress to more severe non-alcoholic steatohepatitis (NASH). NASH is characterized by inflammation and dysregulation of liver-adipose crosstalk with diet being a major factor in its disease etiology and treatment. Apple pomace, an apple processing byproduct, is polyphenol-rich, suggesting potential as a functional food to alleviate features of NASH. Growing (age 22-29 days) female Sprague-Dawley rats were randomly assigned ( $\mathrm{n}=8$ rats/group) to consume purified AIN-93G, AIN-93G/10\% g/kg caloric substitution with apple pomace (AIN/AP), Western diet, or Western/10\% apple pomace (Western/AP) diets for 8 weeks. Rats consuming Western diet had the highest histological evidence inflammation. Hepatic palmitic, palmitoleic, and oleic acid were higher in rats consuming Western diet $(p<0.05)$; whereas with adipose palmitic, stearic, and oleic acid lower $(p<0.01)$ compared to rats consuming Western/AP. Hepatic and adipose gene expression of nuclear transcription factor kappa $B(N F K B)$ was significantly upregulated in rats fed a Western diet compared to all groups and interlekin-6 (IL-6) was significantly upregulated compared to rats consuming AIN diets. Adipose tumor-necrosis factor- $\alpha$ (TNF- $\alpha$ ) was significantly upregulated in rats fed Western diet compared to all diet groups. Apple pomace consumption upregulated $(p<0.01)$ hepatic expression of glutathione peroxidase $(\mathrm{GPx})$. Serum total antioxidants were highest $(p<0.04)$ in rats fed Western/AP, and apple pomace attenuated decreased urinary total antioxidants in rats consuming Western diet. Based on the study results, apple pomace attenuated Western-diet induced changes to NASH by attenuating increased fatty acid crosstalk, upregulated proinflammatory gene expression, and decreased in antioxidant status. The results provide evidence that apple pomace has the potential to be a sustainable functional food.

Keywords: apple pomace, NAFLD, NASH, inflammation, antioxidant, sustainability, fatty acid 


\subsection{Introduction}

Apples are the most widely consumed fruit in the United States with over half of harvested apples processed into juice, resulting in discarding of pulp, skin, seeds, calyx and stem, collectively referred to as apple pomace [1]. Apple pomace disposal is costly and is an industrial waste product that contributes to environmental pollution [2]. Yet, apple pomace contains nutrients and bioactive compounds that may be used as a nutritional aid for dietinduced metabolic complications [2,3]. Liver manifestation of metabolic syndrome, non-alcoholic fatty liver disease (NAFLD), has become the most prevalent liver disease worldwide and is increasing in children [4-6].

Progression of NAFLD to non-alcoholic steatohepatitis (NASH), a more severe manifestation of NAFLD, is proposed to be a multiple-hit pathogenesis. Contributions to NASH are increased de novo lipogenesis (DNL) leading to lipid oxidation, resulting in formation of reactive oxygen species (ROS) and inflammatory cytokines $[7,8]$. Studies show adipose tissue play a role in the NAFLD progression. Cytokines produced in adipose tissue circulate to the liver and contribute to increased hepatic inflammation [9-12]. Also contributing to NASH are altered adipose triglyceride metabolism and free fatty acid released by adipose acting as stimulators of inflammation and oxidative stress [13-20].

Major dietary contributors to the NAFLD cascade of disease progression to NASH are increased consumption of simple carbohydrates and saturated fat [21]. Additionally, high consumption of omega- 6 polyunsaturated fatty acids ( $n-6$ PUFAs) has been reported to exacerbate liver disease through increased inflammation and oxidative stress [22]. The n-6 PUFA, linoleic acid (LA) can be metabolized to arachidonic acid (ARA) and is also a major fatty acid found in Western diets. ARA provides a substrate for cyclooxygenase synthesis of proinflammatory 2-series eicosanoids that can exacerbate NAFLD through increased inflammation [23]. 
Currently, dietary interventions are the main treatment for $\mathrm{NASH}$, with diets high in antioxidants recommended [24]. Apple pomace is a rich source of antioxidant polyphenols indicating potential to attenuate NASH [2]. However, apple pomace also contains fructose, which can increase uric acid, with subsequent increases in inflammation and oxidative stress [2,25-27].

Therefore, it is important to evaluate the potential of apple pomace to be a safe, nutritious, and beneficial food for human consumption. Apple pomace can be a sustainable food source for a growing population by repurposing processing waste as a functional food which decreases costs associated with disposal and reduces environmental pollution. Previously, we showed Western diet induced NAFLD and apple pomace to attenuate indices of NAFLD [28]. The aim of this study was to determine whether apple pomace can attenuate Western diet induced progression from NAFLD to NASH. Additionally, the study determines whether the fructose content of apple pomace affected liver health in animals consuming a standard 'normal' diet. We hypothesize no detrimental effects on the liver due to fructose content in apple pomace in rats fed normal diet, while changes to hepatic-adipose fatty acid profiles, proinflammatory gene expression, and antioxidant status in rats consuming a Western diet are attenuated by the addition of apple pomace

\subsection{Materials and Methods}

Animals and Diets

Weanling (age 22-29 days) female Sprague-Dawley rats $(n=32)$ were purchased from Harlan-Teklad (Indianapolis, IN). Female rats were used on the basis of their greater susceptibility to liver dysfunction with increased carbohydrate consumption [29]. All animal procedures were approved by the Animal Care and Use Committee at West Virginia University and conducted in accordance with the guidelines of the National Research Council for the Care of Laboratory Animals [30]. Rats were individually caged to measure food intake. Rats were 
housed in cages kept in a room at constant temperature of $21+2^{\circ} \mathrm{C}$ with a $12 \mathrm{~h}$ light/dark cycle throughout acclimation and the study duration.

Following a 7-day acclimation, rats were randomly assigned ( $n=8$ rats/group) to four dietary groups consisting of: 1) standard purified rodent diet, AIN-93G, 2) AIN-93G with 10\% $(\mathrm{g} / \mathrm{kg})$ calorically substituted with freeze-dried apple pomace (AIN/AP), 3) Western diet (45\% fat, $33 \%$ sucrose by kcals), or 4$)$ Western diet with $10 \%(\mathrm{~g} / \mathrm{kg})$ calorically substituted with freezedried apple pomace (Western/AP). Locally sourced apple pomace (varieties Gala and Honey Crisp) was provided by Swilled Dog Hard Cider Company (Franklin, WV) and nutrient composition analysis was performed by Medallion Laboratories (Minneapolis, MN). Total polyphenol content in apple pomace and treatment diets were determined using the FolinCiocalteu method [31]. Formulation of AIN diet after apple pomace addition was adjusted to be isocaloric (3.7-3.8 $\mathrm{kcal} / \mathrm{g})$ and Western diet after apple pomace addition was adjusted to be isocaloric $(4.7 \mathrm{kcal} / \mathrm{g})$. Detailed ingredient composition of experimental diets and apple pomace is provided in Supplementary Table 1. Diets were stored at $-20^{\circ} \mathrm{C}$ until fed.

Rats were provided ad libitum access to their assigned diets and to deionized distilled water $\left(\mathrm{ddH}_{2} \mathrm{O}\right)$ throughout the eight weeks study duration. Food intake was measured, and diets replaced every other day while $\mathrm{ddH}_{2} \mathrm{O}$ was replaced weekly. At the end of eight weeks, rats were fasted overnight then euthanized by carbon dioxide inhalation. The liver was excised, perfused, weighed, and then flash frozen in liquid nitrogen and stored at $-80^{\circ} \mathrm{C}$ until analyzed.

\section{Liver Histology}

The left lateral liver lobe $(n=7-8)$ was removed and immediately fixed in $10 \%$ buffered formalin solution for histological evaluation. Dissected tissues were dehydrated through a series of increasing ethanol concentrations $\left(70-100 \%\right.$ in $\left.\mathrm{ddH}_{2} \mathrm{O}\right)$ then placed in xylene and embedded in paraffin. Sections $(8 \mu \mathrm{m})$ from each block were stained with hematoxylin and eosin. Liver inflammation was determined using a modification of the method described by Kleiner et al [32]. 
An inflammation grade of 0 indicated no inflammation present, where a grade of 1 indicated presence of inflammation. All slides were analyzed using a Nikon TE 2000-S light microscope (Nikon Instruments, New York, NY) at magnification $\times 100$ by a trained investigator blinded to the identity of the groups. Images were captured using a PC interface with Q-Capture imaging software (Quantitative Imaging Corporation, BC, Canada).

Diet and tissue fatty acid composition

Diet, liver tissue, and gonadal adipose tissue samples were extracted according to Bligh and Dyer [33]. Briefly, liver tissue $(1 \mathrm{~g})$, diet $(1 \mathrm{~g})$, and adipose tissue $(0.1 \mathrm{~g})$ were homogenized in Tris/EDTA buffer $(\mathrm{pH}=7.4)$. Quantification of fatty acids was determined by adding $50 \mu \mathrm{L}$ of nonadecanoic acid (19:0) as a standard during the initial weigh of the samples. A chloroform:methanol:acetic acid (2:1:0.15 v/v/v) solution was added to all samples and centrifuged at $900 \mathrm{xg}$ for $10 \mathrm{~min}$ at $10^{\circ} \mathrm{C}$. The bottom chloroform layer was collected and mixed with a chloroform:methanol $(4: 1 \mathrm{v} / \mathrm{v})$ solution and centrifugation repeated. The chloroform layer was collected, filtered, and dried under nitrogen gas.

Extracted lipids were transmethylated according to the method described by Fritsche and Johnston [34]. Briefly, fatty acids were methylated by adding $4 \%$ sulfuric acid in anhydrous methanol to the extracted lipid samples followed by incubation in a $90^{\circ} \mathrm{C}$ water bath for $60 \mathrm{~min}$. Samples were cooled to room temperature, and $\mathrm{dd}_{2} \mathrm{O}$ added. Chloroform was then added to the methylated samples and centrifuged at $900 \times \mathrm{g}$ for $10 \mathrm{~min}$ at $10^{\circ} \mathrm{C}$. The collected chloroform layer was filtered through anhydrous sodium sulfate to remove any remaining water. Fatty acid methyl esters (FAMEs) were dried under nitrogen gas and re-suspended in iso-octane.

FAMEs were analyzed by gas liquid chromatography (CP-3800; Varian, Walnut Creek, CA) using an initial temperature of $140^{\circ} \mathrm{C}$ held for $5 \mathrm{~min}$ and then increased $1^{\circ} \mathrm{C}$ per min to a final temperature of $220^{\circ} \mathrm{C}$. A wall-coated open tubular fused silica capillary column (Varian) was used to separate FAME with CP-Sil 88 at the stationary phase. Nitrogen was used as the 
carrier gas, and total separation time was 85 min. Quantitative 37 Component FAMEs Sigma Mix (Supelco, Bellefonte, PA) was used to identify fatty acids. Fatty acids were determined by retention time and quantified using peak ultra-counts. All samples are performed in duplicates and reported as $\mathrm{mg} / \mathrm{g}$ of total fatty acids.

Liver and Adipose Oxidative Stress and Inflammatory Gene Expression

Liver and gonadal adipose tissue total RNA was extracted from $-80^{\circ} \mathrm{C}$ frozen samples (50 mg) using the Zymo Research Direct-zol RNA Miniprep Plus Isolation Kit (Irvine, CA, catalog \#R2071) and the Qiagen RNeasy Tissue Mini Kit (Venlo, Netherlands, catalog \# 74804), respectively, according to the manufacturer's instruction for total RNA isolation. Isolated RNA integrity was visualized on a 1.5\% agarose gel and quantified by spectrophotometry (NanoDrop 100; Thermo Fisher Scientific, Waltham, MA). Following DNase I treatment with TURBO DNAfree kit (Thermo Fisher Scientific), total mRNA was amplified using the Superscript IV FirstStrand Synthesis System with oligo dT primers (Thermo Fisher Scientific).

Real-time quantitative polymerase chain reaction (RT-qPCR) consisted of $2.5 \mu \mathrm{l}$ of SYBR Green Master Mix (Thermo Fisher), $1 \mu \mathrm{L}$ of cDNA (diluted 1:10), $1 \mu \mathrm{L}$ of respective forward and reverse primers $(10 \mu \mathrm{M})$ and $0.5 \mu \mathrm{l}$ of deionized distilled water for a total reaction volume of $5 \mu \mathrm{l}$. The reactions were performed in a $7500 \mathrm{ABI}$ Real-Time PCR System (Thermo Fisher Scientific). The thermal profile consisted of $50^{\circ} \mathrm{C}$ for $2 \mathrm{~min}, 95^{\circ} \mathrm{C}$ for 10 min then 40 cycles of $95^{\circ} \mathrm{C}$ for $15 \mathrm{sec}$ and $60^{\circ} \mathrm{C}$ for $1 \mathrm{~min}$. A melt curve analysis was applied at the end of cycling. Primers that were designed for transcription factor and genes regulating inflammation included: nuclear factor kappa-light chain enhancer of $B$ cells (NFKB), tumor necrosis factoralpha (TNF- $\alpha$ ), interleukin-6 (IL-6), interleukin-10 (IL-10). Genes regulating PUFA metabolism/inflammation included: cyclooxygenase 1 (COX1), cyclooxygenase 2 (COX2), arachidonate 5-lipoxygnease (5LOX). Genes regulating oxidation and antioxidants included: NADPH oxidase 4 (NOX4), transforming growth factor beta-3 (TGFB3), chemokine (CC-motif) 
ligand-2 (CCL-2), nuclear factor-like 2 (Nrf-2), superoxide dismutase 1 (SOD-1), superoxide dismutase 2 (SOD-2), catalase, and glutathione peroxidase (GPx), as well as for housekeeping genes $\beta$-actin and glyceraldehyde 2-phosphate dehydrogenase (GAPDH). Primers were designed using the Primer3 online program (Howard Hughes Medical Institute) and respective mRNA sequences were obtained at the NCBI RefSeq RNAs catalog through gene ID numbers. Forward and reverse primers for gene transcriptions are listed in Supplementary Table 2.

Tissue oxidation and antioxidant measurements

Hydrogen peroxide content in the liver was determined using a commercially available assay kit (Abcam, Cambridge, MA). Briefly, liver samples $(50 \mathrm{mg})$ were homogenized, centrifuged at $10,000 \times \mathrm{g}$ for $2 \mathrm{~min}$ at $4^{\circ} \mathrm{C}$, and supernatant collected. Deproteinization was performed by addition of $4 \mathrm{M}$ perchloric acid (PCA), followed by precipitation of excess PCA with $2 \mathrm{M}$ potassium hydroxide. Liver samples were adjusted to $\mathrm{pH}$ 6.5-8. Absorbance was read at $570 \mathrm{~nm}$ using a BioTek Epoch microplate spectrophotometer (Walooski, VT). Inter-assay coefficient of variation was $12.7 \%$.

Thiobarbituric acid reactive substances (TBARS) content in the liver was determined by measuring malondialdehyde (MDA) using a commercially available assay (Cayman Chemical, Ann Arbor, MI). Briefly, liver samples (25 mg) were homogenized, centrifuged at $1600 \times \mathrm{g}$ for 10 $\min$ at $4^{\circ} \mathrm{C}$, and supernatant collected. Liver samples were boiled at $100^{\circ} \mathrm{C}$ for one hour and placed on ice for $10 \mathrm{~min}$ to stop the reaction. Absorbance was read at $535 \mathrm{~nm}$ using a BioTek Epoch microplate spectrophotometer. Inter-assay coefficient of variation was $15.2 \%$.

Total polyphenol content in the liver was determined according to the Folin-Ciocalteu method (Blainski et al. 2013). The inter-assay coefficient of variation was $11.2 \%$.

Serum and urinary total antioxidant measurements 
Serum and urinary total antioxidant capacity was determined by commercially available antioxidant assay kit (Cayman Chemical, Ann Arbor, MI). At the end of the feeding study, rats were individually housed for $24 \mathrm{~h}$ in metabolic cages to collect urine. Ascorbic acid $(0.1 \%)$ was added to the urine collection tube as a preservative along with $1 \mathrm{ml}$ of mineral oil to prevent evaporation. Collected urine samples were centrifuged for $10 \mathrm{~min}$ at $1500 \mathrm{xg}$ at $4^{\circ} \mathrm{C}$ to remove debris. Fasted blood was collected by aorta puncture. Collected blood was centrifuged at $1500 \mathrm{x}$ $\mathrm{g}$ for $10 \mathrm{~min}$ at $4^{\circ} \mathrm{C}$ to obtain serum. Serum and urine samples were stored at $-80^{\circ} \mathrm{C}$ until assayed for total antioxidant capacity. Briefly, serum and urinary samples were diluted 1:20 and $40 \mu \mathrm{L}$ hydrogen peroxide $(441 \mu \mathrm{M})$ working solution added. Absorbance was read at $750 \mathrm{~nm}$ using a BioTek Epoch microplate spectrophotometer. Inter-assay coefficient of variation was $14.5 \%$ for serum samples and $21.1 \%$ for urine samples.

Serum and urinary biochemical measurements

Serum measurement of liver function and damage included: alanine aminotransferase (ALT), aspartate aminotransferase (AST), total bilirubin, and albumin were determined enzymatically using a commercially available Vet-16 rotor and quantified by a Hemagen Analyst automated spectrophotometer (Hemagen Diagnostics Inc., Columbia, MD). AST:ALT ratio was determined by dividing AST values by ALT values.

Serum and urine uric acid was determined by commercially available enzymatic assay (Cayman Chemical). Briefly, serum and urine samples were aliquoted onto a 96-well plate and incubated for 15 minutes. Reaction was initiated by adding $15 \mu \mathrm{L}$ of uricase and horseradish peroxidase enzyme mixture, and read at an excitation of $535 \mathrm{~nm}$ and an emission of $590 \mathrm{~nm}$ using a BioTek Synergy H1 microplate reader (Winooski, VT). Inter-assay coefficient of variation was $32.1 \%$ for both serum and urine.

Statistics 
Results are expressed as mean \pm standard error of the mean (SEM). Gene expression was determined as a function of $m R N A$ abundance $(A)$, where $A=1 /$ (gene of interest's primer efficiency $x \Delta C T$ (g.o.i.))- (average housekeeping's primer efficiency $x \Delta C T$ (h.k.)), where the product of efficiency and average of expression of $\beta$-actin was averaged with the product of efficiency and average of expression of GAPDH to determine the overall expression of the two housekeeping genes $[28,35,36]$. Gene expression data for each treatment group were logtransformed prior to statistical analysis. One-way ANOVA was used to determine differences among diet groups. Post hoc multiple comparison tests were performed using Tukey's test with treatment differences considered significant at $p<0.05$ and a tendency at $p<0.08$. All statistical analyses were performed using JMP 12.2 statistical software package (SAS Institute, Cary, $\mathrm{NC})$.

\subsection{Results}

Diet Analysis

As shown in Supplementary Table 1, caloric replacement with apple pomace in AIN and Western diets resulted in higher total polyphenols. Fat content was higher in the Western diets than standard AIN diets. Shown in Table 1, Western diets had significantly higher saturated fatty acids (SFAs), palmitic (16:0) and stearic acid (18:0) than AIN diets. Western/AP diet had the highest $(p<0.0001)$ palmitic acid. Western diets were also significantly higher in monounsaturated fatty acids (MUFAs), palmitoleic acid (16:1n 7), and oleic acid (18:1n 9), than AIN diets.

Essential fatty acids, n-6 PUFA, LA (18:2n-6), and n-3 PUFA, $\alpha$-linoleic acid (ALA, 18:3n-3) content were lower $(p<0.0001)$ in Western diets than AIN diets. Long chain $n-6$ PUFA, ARA was higher $(p<0.0001)$ in Western diets as compared to AIN diets, which contained negligible amounts. There were no detectable levels of long chain n-3 PUFAs, eicosapentaenoic acid (EPA, 20:5n-3) or docosahexaenoic acid (DHA, 22:6n-3) in any of the diets. 
Caloric intake, body weight, and tissue weights

Shown in Table 2, rats consuming the Western diets consumed more $(p<0.0001)$ fat than rats consuming AIN diets. But, rats fed the AIN diets consumed more $(p<0.0001)$ carbohydrates. Overall, rats fed the Western diets consumed significantly more calories than rats fed the AIN diets. No significant differences were observed in body weight gain, but a tendency $(p=0.08)$ for heavier final body weight in rats fed Western diets. Rats fed the Western diets had heavier $(p<0.0001)$ gonadal adipose tissue than rats fed the AIN diets.

Liver histological evaluation

As shown in Figure 1, 88\% of rats fed Western diet and $63 \%$ of rats fed Western/AP diet had evidence of hepatic inflammation. AIN and AIN/AP diet groups each showed $15 \%$ of animals having hepatic inflammation.

Liver and gonadal adipose fatty acid composition

Western/AP diet contained the highest amount of dietary palmitic acid (Table 1), but hepatic palmitic acid content was not significantly different than rats consuming the AIN diet (Table 3). Both Western diets contained higher amounts of stearic acid but only showed a tendency $(p=0.08)$ for higher hepatic stearic acid content compared to rats fed AIN diets. Western diets also contained higher amounts of palmitoleic and oleic acid than AIN diets. Rats consuming Western diet had the highest $(p=0.05)$ hepatic palmitoleic acid content. Rats fed Western diet, but not Western/AP diet, had higher hepatic oleic acid content $(p=0.0005)$ compared to rats fed the AIN diets. Rats consuming Western diets had lower $(p<0.0001)$ hepatic n-6 PUFAs, LA and ALA content when compared to rats consuming the AIN diets, but no difference in hepatic ARA content was observed among diet groups. No EPA or DHA was found in diet or the liver of any of the diet groups. 
Gonadal adipose tissue palmitic and stearic acids were higher $(p<0.0008)$ in rats consuming AIN/AP and Western/AP diets than rats consuming AIN and Western diets. Although no differences in gonadal adipose tissue palmitoleic acid content were observed among diet groups, substitution of diets with apple pomace resulted in higher $(p=0.01)$ gonadal adipose tissue oleic acid. Gonadal adipose tissue LA was higher $(p<0.0001)$ in rats consuming AIN/AP than the Western diets. Additionally, gonadal adipose tissue ALA was highest $(p<0.0001)$ in rats fed AIN/AP diet. ARA and EPA were below detected in the gonadal adipose tissue in any diet group. However, gonadal adipose tissue contained more DHA $(p=0.008)$ in rats fed AIN/AP than Western diets.

Hepatic and gonadal adipose gene markers for inflammation and oxidative stress

As shown in Figure 2A, rats consuming Western diet, but not Western/AP diet significantly upregulated hepatic transcription factor, NFKB and inflammatory cytokine, IL-6 compared to rats fed the AIN diets. No significant differences were observed in hepatic gene expression of inflammatory cytokines, TNF- $\alpha$ and IL-10, among diet groups

As shown in Figure 2B, gonadal adipose tissue NFKB, TNF $\alpha$ and IL-6 gene expression was upregulated $(p \leq 0.05)$ in rats consuming Western diet, but not Western/AP diet compared to rats fed the AIN diets. No significant differences were observed in gonadal adipose tissue gene expression of IL-10 among diet groups.

Measurements of PUFA metabolism, inflammation and ROS

As shown in Figure 3A, no significant differences were observed in hepatic gene expression of COX1, COX2, 5-LOX, or NOX4 among diet groups. As shown in Figure 3B, no significant differences were observed in gonadal adipose tissue gene expression of COX1, COX2, 5LOX, or NOX4 among diet groups. As shown in Figure 4, there were no significant differences in hepatic expression of genes promoting progression of NAFLD to NASH, TGF 33 
or CCL-2. There were no significant differences in hepatic gene expression of antioxidant defense transcription factor, Nrf2 or endogenous antioxidant enzymes, SOD1, SOD2, or catalase among diet groups. However, rats consuming diets substituted with apple pomace showed upregulation $(p<0.0001)$ hepatic GPx gene expression.

\section{Oxidative Stress and Antioxidant Status Measurements}

No significant differences were observed among diet groups for liver oxidation products, hydrogen peroxide (Figure 5A) or MDA (Figure 5B). For antioxidants, there were no significant differences in total polyphenols in the liver among diet groups despite higher diet content with apple pomace caloric replacement (Figure 5C). However, rats consuming Western/AP diet had the highest $(p<0.05)$ serum total antioxidants (Figure 5D) while rats consuming Western diet had $(p<0.0004)$ the lowest urinary total antioxidants (Figure 5E).

Serum and urine biochemical measurements

As shown in Table 4, there were no significant differences for serum markers of liver function and damage: ALT, AST, AST:ALT ratio, bilirubin, or albumin among diet groups. Also, there were no significant differences in serum or urine uric acid among diet groups.

\subsection{Discussion}

Previously, we showed caloric substitution with $10 \%$ apple pomace in rats consuming a Western diet attenuated increased histological evidence of steatosis, hepatic triglyceride content, and hepatic expression of the enzyme DGAT2, which catalyzes the terminal step in triglyceride synthesis [28]. To extend this study, we evaluated the effects of apple pomace consumption on diet-induced progression of NAFLD to NASH. The current study showed apple pomace attenuated histological evidence of inflammation in the liver of rats consuming a 
Western diet. Previous studies showed adipose tissue can alter liver fatty acid composition and in turn, gene expression of inflammatory cytokines. Additionally, fatty acids released from the adipose can contribute to hepatic inflammation $[37,38]$. In our study, rats consuming Western diets had higher $(p<0.0001)$ gonadal adipose weight compared to rats consuming standard AIN diets. Further, lipidomic analysis of individuals with NASH-associated hepatocellular carcinoma showed a significant increase in hepatic MUFA content [39].

In this study, both Western diets contained more MUFAs; however, rats consuming Western diet, but not Western/AP, had higher hepatic MUFAs, palmitoleic acid $(p=0.05)$ and oleic acid $(p=0.0005)$ content compared to rats consuming AIN diets. Additionally, gonadal adipose tissue of rats consuming Western diet, but not Western/AP diet had reduced $(p=0.01)$ oleic acid content compared to AIN/AP diet. High hepatic oleic acid content, and low adipose oleic acid content suggests liver-adipose crosstalk, where oleic acid released from adipose tissue was deposed in the liver. MUFA crosstalk between liver and adipose tissue has been shown to regulate DNL and inflammation [40-42]. In our study, higher MUFA transport from gonadal adipose to the liver in rats consuming Western diet was attenuated by caloric substitution with $10 \%$ apple pomace. Increased MUFAs influence triglyceride synthesis and liver steatosis, promoting NAFLD, where increased SFAs stimulate inflammation, promoting progression to NASH $[16,43]$.

Rats consuming Western diet, but not Western/AP had higher $(p=0.0007)$ hepatic SFA, palmitic acid, content compared to rats consuming AIN diets, despite Western/AP diet containing the highest dietary amount of SFA. Additionally, gonadal adipose tissue of rats consuming Western diet had lower $(p<0.0008)$ palmitic and stearic acid content than rats consuming Western/AP diets. The results suggest rats consuming the Western diet had increased SFA crosstalk, where palmitic acid released from gonadal adipose was transported to the liver, with apple pomace once again attenuating this increase in the liver. Studies have 
implicated SFAs in NASH due to increased SFA content promoting gene expression of proinflammatory cytokines $[44,45]$.

Transcription factor, NFKB is a key regulator of numerous inflammatory cytokines [46]. Upregulated gene expression of NFKB and cytokine, IL-6, are major factors in the progression of NAFLD to NASH $[21,47,48]$. In the current study, rats fed Western diet had the highest upregulated $(p<0.05)$ gene expression of NFKB and IL-6 in liver and gonadal adipose tissue. Additionally, rats consuming a Western diet also had the highest upregulation $(p=0.001)$ of gene expression of TNF- $\alpha$ in gonadal adipose tissue. According to Hotamisligil, et al [49]., adipose tissue is a major production site for TNF- $\alpha$. Studies have shown free fatty acids alter gene expression of cytokines resulting in progression liver disease $[16,41,44,50]$. Caloric substitution with $10 \%$ apple pomace attenuated liver deposition of MUFAs and SFAs released from gonadal adipose tissue and upregulation of gene expression of inflammatory cytokines induced by Western diet resulting in the absence of development of NAFLD and progression to NASH in rats fed Western/AP diet.

PUFAs also regulate inflammatory gene expression and in turn, influence liver disease progression [22,51]. AIN diets contained more n-6 PUFA, LA, than Western diets, which in turn increased $(p<0.0001)$ hepatic and gonadal adipose tissue LA content. Long-chain n-6 PUFA, ARA, content was higher in Western diets compared to AIN diets, but no significant differences were observed in hepatic ARA content. This may be due to higher LA content in AIN diets undergoing metabolism in the liver to ARA [52]. ARA was not detectable in gonadal adipose tissue, which was expected since ARA is primarily stored in muscle and liver [53]. ALA, a n-3 PUFA, content was significantly higher In AIN diets than Western diets. Rats fed AIN/AP had the highest $(p<0.001)$ adipose tissue ALA. Additionally, gonadal adipose tissue of rats consuming AIN/AP had higher $(p=0.0078)$ DHA content compared to rats consuming Western diets. In the absence of dietary DHA in any of the diets, this was likely due to higher dietary ALA in AIN/AP diet being metabolized in adipose tissue to long-chain n-3 PUFA, DHA (Table 3) [54]. 
ARA tissue composition influences inflammation by acting as a substrate for COX- and LOXmediated pathways, producing proinflammatory eicosanoids and leukotrienes, respectively $[22,55]$. n-3 PUFAs compete for the same COX and LOX enzymes to produce less inflammatory eicosanoids and leukotrienes [56]. Despite changes in tissue fatty acid composition, there were no significant differences in COX1, COX2, or 5LOX gene expression in liver or gonadal adipose tissue among diet groups.

Alterations in tissue fatty acid composition can also promote lipid peroxidation and increased oxidative stress, which are suggested to promote NASH $[17,57,58]$. In the present study, no significant differences were observed in hepatic prooxidants hydrogen peroxide and MDA content among diet groups. Upregulated gene expression of NOX4, TGF $\beta 3$, and CCL-2 are associated with NASH and increased oxidative stress [59-62]. Our study also showed no significant differences in gene expression of hepatic or adipose NOX4, or hepatic TGF $\beta 3$ or CCL-2 among diet groups (Figure 4). Oxidative stress occurs due to an imbalance of ROS and antioxidants, indicating antioxidant status as critical in attenuation of NASH [63]. Our study showed no significant differences in hepatic gene expression of transcription factor, Nrf2, a key regulator of endogenous antioxidant enzymes, SOD1, SOD2, or catalase among diet groups. However, rats consuming AIN and Western diets containing apple pomace had upregulated $(p<0.0001)$ hepatic expression of GPx. Endogenous antioxidant, GPx defends against increased oxidative stress due to lipid peroxidation [64]. Diets with apple pomace contained more polyphenols. Increased dietary antioxidants have been shown to increase GPx gene expression [65]. Decreases in antioxidant enzyme activity, specifically glutathione enzymes, and diminished antioxidant response has been reported in subjects with NASH. [66,67]. In the present study, there were no significant differences in liver polyphenol content among diet groups, which was expected, as polyphenols circulate in the blood with excess polyphenols being excreted, rather than depose in tissues [68,69]. Higher antioxidant status with apple pomace caloric consumption was indicated by highest $(p=0.0001)$ serum total antioxidants in 
rats consuming Western/AP and lowest $(p=0.0004)$ urinary total antioxidant excretion in rats consuming Western diet. These results showed apple pomace increased antioxidant bioavailability, which can result in attenuation of $\mathrm{NASH}[70]$.

Bobek, et al [71]. reported rats fed cholesterol diets (0.3\%) supplemented with $5 \%$ apple pomace for 10 weeks reduced erythrocyte SOD, catalase, and GPx. Another study reported feeding rats a standard diet with 14-15\% apple pomace for 4 weeks decreased hepatic MDA and increase erythrocyte SOD and serum antioxidant capacity [72]. However, neither feeding study investigated apple pomace's ability to attenuate diet-induced NASH. Most studies investigating antioxidant effects of apple pomace used polyphenols isolated from apple pomace. Consuming isolated bioactive isolated from apple pomace versus whole apple pomace avoids fructose intake. Fructose overconsumption has been shown to increase uric acid resulting in increases in proinflammatory cytokine and oxidative stress [25,73]. Our study showed no significant effect of caloric substation of AIN diet with apple pomace on serum or urinary uric acid, expression of proinflammatory cytokines, or indices of oxidative stress. Additionally, caloric substitution of Western diet with apple pomace attenuated progression to NASH. The results indicate the fructose content in $10 \%$ apple pomace to be safe for consumption. Apple pomace also contains a substantial amount of dietary fiber, which has been shown to produce synergistic effects when consumed with polyphenols [74-77]. Purification of bioactive components from pomace is both time consuming and costly, providing further rationale for consuming whole apple pomace [78-80].

In conclusion, Western diet containing apple pomace ameliorated palmitic and oleic acid transport from adipose and deposition in the liver, upregulation of inflammatory genes in liver and gonadal adipose, and improved antioxidant status. Further, caloric substitution of standard AIN diet with $10 \%$ apple pomace rats showed the fructose content of apple pomace did not promote detrimental liver effects. Based on the current animal study, absence of detrimental effect on liver health while attenuating NAFLD progression to NASH induced by Western diet 
consumption indicates apple pomace is a potential safe, beneficial, and sustainable functional food for human consumption.

Conflicts of interest

There are no conflicts to declare.

Acknowledgments

The authors would like to thank Swilled Dog Hard Cider Company for the donation of apple pomace used in this study. This research was funded by Hatch WVA 1017641 and the Davis College Dean's discretionary fund. 


\subsection{References}

1. USDA ERS - Food Availability and Consumption. USDA Economic Research Service . https://www.ers.usda.gov/data-products/ag-and-food-statistics-charting-the-essentials/foodavailability-and-consumption/. Published 2017. Accessed June 1, 2018.

2. Bhushan S, Kalia K, Sharma M, Singh B, Ahuja PS. Processing of apple pomace for bioactive molecules. Crit Rev Biotechnol. 2008;28(4):285-296.

doi:10.1080/07388550802368895

3. Grigoras C, Destandau E, Fougere L, Elfakir C. Evaluation of apple pomace extracts as a source of bioactive compounds. Ind Crops Prod. 2013;49:794-804. doi:10.1016/J.INDCROP.2013.06.026

4. Kleiner DE, Behling C, Brunt EM, et al. Comparison of adult and pediatric NAFLDconfirmation of a second pattern of progressive fatty liver disease in children: 189. Hepatology. 2006;44:259A-260A. https://insights.ovid.com/hepatology/hepa/2006/10/001/comparison-adultpediatric-nafld-confirmation/189/01515467.

5. Loomba R, Sanyal AJ. The global NAFLD epidemic. Nat Rev Gastroenterol Hepatol. 2013;10(11):686-690. doi:10.1038/nrgastro.2013.171

6. Anderson EL, Howe LD, Jones HE, Higgins JPT, Lawlor DA, Fraser A. The prevalence of non-alcoholic fatty liver disease in children and adolescents: a systematic review and metaanalysis. Wong V, ed. PLoS One. 2015;10(10):e0140908. doi:10.1371/journal.pone.0140908

7. Suzuki A, Diehl AM. Nonalcoholic Steatohepatitis. Annu Rev Med. 2017;68(1):85-98. doi:10.1146/annurev-med-051215-031109

8. Basaranoglu M, Basaranoglu G, Bugianesi E. Carbohydrate intake and nonalcoholic fatty liver disease: fructose as a weapon of mass destruction. Hepatobiliary Surg Nutr. 2015;4(2):109-116. doi:0.3978/j.issn.2304-3881.2014.11.05 
9. Qureshi K, Abrams GA. Metabolic liver disease of obesity and role of adipose tissue in the pathogenesis of nonalcoholic fatty liver disease. World J Gastroenterol. 2007;13(26):35403553. doi:10.1002/hep.25539

10. Sampey BP, Vanhoose AM, Winfield HM, et al. Cafeteria Diet Is a Robust Model of Human Metabolic Syndrome With Liver and Adipose Inflammation: Comparison to High-Fat Diet. Obesity. 2011;19(6):1109-1117. doi:10.1038/oby.2011.18

11. Lallukka S, Sevastianova K, Perttilä J, et al. Adipose tissue is inflamed in NAFLD due to obesity but not in NAFLD due to genetic variation in PNPLA3. Diabetologia. 2013;56(4):886892. doi:10.1007/s00125-013-2829-9

12. Tilg $\mathrm{H}$, Moschen AR. Evolution of inflammation in nonalcoholic fatty liver disease: The multiple parallel hits hypothesis. Hepatology. 2010;52(5):1836-1846. doi:10.1002/hep.24001

13. James MJ, Gibson RA, Cleland LG. Dietary polyunsaturated fatty acids and inflammatory mediator production. Am J Clin Nutr. 2000;71(1):343s-348s. doi:10.1093/ajen/71.1.343s

14. Farrell GC, Larter CZ. Nonalcoholic fatty liver disease: From steatosis to cirrhosis. Hepatology. 2006;43(S1):S99-S112. doi:10.1002/hep.20973

15. Tilg $\mathrm{H}$, Moschen AR. Evolution of inflammation in nonalcoholic fatty liver disease: The multiple parallel hits hypothesis. Hepatology. 2010;52(5):1836-1846. doi:10.1002/hep.24001

16. Milanski M, Degasperi G, Coope A, et al. Saturated fatty acids produce an inflammatory response predominantly through the activation of TLR4 signaling in hypothalamus: implications for the pathogenesis of obesity. J Neurosci. 2009;29(2):359-370.

doi:10.1523/JNEUROSCI.2760-08.2009 
17. Day CP, James OFW. Steatohepatitis: A tale of two "hits"? Gastroenterology. 1998;114(4):842-845. doi:10.1016/S0016-5085(98)70599-2

18. Piro S, Anello M, Di Pietro C, et al. Chronic exposure to free fatty acids or high glucose induces apoptosis in rat pancreatic islets: possible role of oxidative stress. Metabolism. 2002;51(10):1340-1347. doi:10.1053/META.2002.35200

19. Anitha Nandhini AT, Balakrishnan SD, Anuradha C V. Taurine modulates antioxidant potential and controls lipid peroxidation in the aorta of high fructose-fed rats. J Biochem Mol Biol Biophys. 2002;6(2):129-133. doi:10.1080/10258140290027261

20. Videla LA, Rodrigo R, Araya J, Poniachik J. Oxidative stress and depletion of hepatic long-chain polyunsaturated fatty acids may contribute to nonalcoholic fatty liver disease. Free Radic Biol Med. 2004;37(9):1499-1507. doi:10.1016/J.FREERADBIOMED.2004.06.033

21. Buzzetti E, Pinzani M, Tsochatzis EA. The multiple-hit pathogenesis of non-alcoholic fatty liver disease (NAFLD). Metabolism. 2016;65(8):1038-1048.

doi:10.1016/J.METABOL.2015.12.012

22. Simopoulos AP. An increase in the omega-6/omega-3 fatty acid ratio increases the risk for obesity. Nutrients. 2016;8(3):128. doi:10.3390/nu8030128

23. French SW, Morimoto M, Reitz RC, et al. Lipid Peroxidation, CYP2E1 and Arachidonic Acid Metabolism in Alcoholic Liver Disease in Rats. J Nutr. 1997;127(5):907S-911S. doi:10.1093/jn/127.5.907S

24. Adams LA, Angulo P. Treatment of non-alcoholic fatty liver disease. Postgrad Med J. 2006;82(967):315-322. doi:10.1055/s-2001-12931 
25. Nakagawa T, Hu H, Zharikov S, et al. A causal role for uric acid in fructose-induced metabolic syndrome. Am J Physiol Physiol. 2006;290(3):F625-F631. doi:10.1152/ajprenal.00140.2005

26. Mosca A, Nobili V, De Vito R, et al. Serum uric acid concentrations and fructose consumption are independently associated with NASH in children and adolescents. J Hepatol. 2017;66(5):1031-1036. doi:10.1016/J.JHEP.2016.12.025

27. Lim JS, Mietus-Snyder M, Valente A, Schwarz J-M, Lustig RH. The role of fructose in the pathogenesis of NAFLD and the metabolic syndrome. Nat Rev Gastroenterol Hepatol. 2010;7(5):251-264. doi:10.1038/nrgastro.2010.41

28. Skinner R, Warren D, Lateef S, et al. Apple Pomace Consumption Favorably Alters Hepatic Lipid Metabolism in Young Female Sprague-Dawley Rats Fed a Western Diet. Nutrients. 2018;10(12):1882. doi:10.3390/nu10121882

29. Vilà L, Roglans N, Perna V, et al. Liver AMP/ATP ratio and fructokinase expression are related to gender differences in AMPK activity and glucose intolerance in rats ingesting liquid fructose. J Nutr Biochem. 2011;22(8):741-751. doi:10.1016/J.JNUTBIO.2010.06.005

30. National Research Council. Guide for the Care and Use of Laboratory Animals: Eighth Edition - National Research Council, Division on Earth and Life Studies, Institute for Laboratory Animal Research, Committee for the Update of the Guide for the Care and Use of Laboratory Animals - Google Books. 8th ed. Washington D.C.; 2010. https://books.google.com/books?hl=en\&lr=\&id=Vp5mgXtxYdQC\&oi=fnd\&pg=PP2\&dq=national+ research+council+2010+rats\&ots=FrTgd1JCl5\&sig=eZ_vGWk36QfLScXnMbS_BgT8XU\#v=on epage\&q=national research council 2010 rats\&f=false. Accessed February 27, 2018. 
31. Blainski A, Lopes G, de Mello J, Blainski A, Lopes GC, de Mello JCP. Application and Analysis of the Folin Ciocalteu Method for the Determination of the Total Phenolic Content from Limonium Brasiliense L. Molecules. 2013;18(6):6852-6865. doi:10.3390/molecules18066852

32. Kleiner DE, Brunt EM, Van Natta M, et al. Design and validation of a histological scoring system for nonalcoholic fatty liver disease. Hepatology. 2005;41(6):1313-1321. doi:10.1002/hep.20701

33. Bligh EG, Dyer WJ. A rapid method of total lipid extraction and purification. Can J Biochem Physiol. 1959;37(1):911-917. doi:10.1139/059-099

34. Fritsche $\mathrm{KL}$, Johnston $\mathrm{P}$ V. Effect of dietary a-linolenic acid on growth, metastasis, fatty acid profile and prostaglandin production of two murine mammary adenocarcinomas. J Nutr. 1990;120(12):1601-1609. doi:10.1093/jn/120.12.1601

35. Jacometo CB, Schmitt E, Pfeifer LFM, et al. Linoleic and a-linolenic fatty acid consumption over three generations exert cumulative regulation of hepatic expression of genes related to lipid metabolism. Genes Nutr. 2014;9(4):405. doi:10.1007/s12263-014-0405-7

36. Maditz KH, Benedito VA, Oldaker C, et al. Feeding Soy Protein Isolate and n-3 PUFA Affects Polycystic Liver Disease Progression in a PCK Rat Model of Autosomal Polycystic Kidney Disease. J Pediatr Gastroenterol Nutr. 2015;60(4):467-473.

doi:10.1097/MPG.0000000000000649

37. Donnelly KL, Smith CI, Schwarzenberg SJ, Jessurun J, Boldt MD, Parks EJ. Sources of fatty acids stored in liver and secreted via lipoproteins in patients with nonalcoholic fatty liver disease. J Clin Invest. 2005;115(5):1343-1351. doi:10.1172/JCI23621 
38. Roberts AA, Hebbard LW. Molecular cross-talk between the liver and white adipose tissue links excessive noURIshment to hepatocellular carcinoma. Transl Cancer Res. 2016;5(6):S1222-S1226. doi:10.21037/10460

39. Muir K, Hazim A, He Y, et al. Proteomic and lipidomic signatures of lipid metabolism in NASH-associated hepatocellular carcinoma. Cancer Res. 2013;73(15):4722-4731. doi:10.1158/0008-5472.CAN-12-3797

40. Burhans MS, Ntambi JM. Monounsaturated Fatty Acid Mediated Liver-Adipose Tissue Crosstalk and Metabolic Regulation. In: Hepatic De Novo Lipogenesis and Regulation of Metabolism. Cham: Springer International Publishing; 2016:255-265. doi:10.1007/978-3-31925065-6_12

41. Malhi H, Gores GJ. Molecular mechanisms of lipotoxicity in nonalcoholic fatty liver disease. Semin Liver Dis. 2008;28(4):360-369. doi:10.1055/s-0028-1091980

42. Saponaro C, Gaggini M, Carli F, et al. The Subtle Balance between Lipolysis and Lipogenesis: A Critical Point in Metabolic Homeostasis. Nutrients. 2015;7(11):9453-9474. doi:10.3390/nu7115475

43. Miyazaki M, Kim YC, Ntambi JM. A lipogenic diet in mice with a disruption of the stearoyl-CoA desaturase 1 gene reveals a stringent requirement of endogenous monounsaturated fatty acids for triglyceride synthesis. J Lipid Res. 2001;42(7):1018-1024.

44. Leamy AK, Egnatchik RA, Young JD. Molecular mechanisms and the role of saturated fatty acids in the progression of non-alcoholic fatty liver disease. Prog Lipid Res. 2013;52(1):165-174. doi:10.1016/J.PLIPRES.2012.10.004 
45. Nestel P, Clifton P, Noakes M. Effects of increasing dietary palmitoleic acid compared with palmitic and oleic acids on plasma lipids of hypercholesterolemic men. J Lipid Res. 1994;35(4):656-662. http://www.ncbi.nlm.nih.gov/pubmed/8006520.

46. Tak PP, Firestein GS. NF-kappaB: a key role in inflammatory diseases. J Clin Invest. 2001;107(1):7-11. doi:10.1172/JCl11830

47. Michelotti GA, Machado M V., Diehl AM. NAFLD, NASH and liver cancer. Nat Rev Gastroenterol Hepatol. 2013;10(11):656-665. doi:10.1038/nrgastro.2013.183

48. Wieckowska A, Papouchado BG, Li Z, Lopez R, Zein NN, Feldstein AE. Increased Hepatic and Circulating Interleukin-6 Levels in Human Nonalcoholic Steatohepatitis. Am J Gastroenterol. 2008;103(6):1372-1379. doi:10.1111/j.1572-0241.2007.01774.x

49. Hotamisligil GS, Arner P, Caro JF, Atkinson RL, Spiegelman BM. Increased adipose tissue expression of tumor necrosis factor-alpha in human obesity and insulin resistance. J Clin Invest. 1995;95(5):2409-2415. doi:10.1172/JCl117936

50. van Dijk SJ, Feskens EJ, Bos MB, et al. A saturated fatty acid-rich diet induces an obesity-linked proinflammatory gene expression profile in adipose tissue of subjects at risk of metabolic syndrome. Am J Clin Nutr. 2009;90(6):1656-1664. doi:10.3945/ajcn.2009.27792

51. Wang X, Cao Y, Fu Y, Guo G, Zhang X. Liver fatty acid composition in mice with or without nonalcoholic fatty liver disease. Lipids Health Dis. 2011;10:234. doi:10.1186/1476-511X$10-234$

52. Salem N, Pawlosky R, Wegher B, Hibbeln J. In vivo conversion of linoleic acid to arachidonic acid in human adults. Prostaglandins, Leukot Essent Fat Acids. 1999;60(5-6):407410. doi:10.1016/S0952-3278(99)80021-0 
53. Smith GI, Atherton $\mathrm{P}$, Reeds $\mathrm{DN}$, et al. Omega-3 polyunsaturated fatty acids augment the muscle protein anabolic response to hyperinsulinaemia-hyperaminoacidaemia in healthy young and middle-aged men and women. Clin Sci (Lond). 2011;121(6):267-278.

doi:10.1042/CS20100597

54. Todorčević M, Hodson L. The Effect of Marine Derived n-3 Fatty Acids on Adipose Tissue Metabolism and Function. J Clin Med. 2015;5(1). doi:10.3390/jcm5010003

55. Nanji A, Miao L, Thomas $\mathrm{P}$, et al. Enhanced cyclooxygenase-2 gene expression in alcoholic liver disease in the rat. Gastroenterology. 1997;112(3):943-951. doi:10.1053/gast.1997.v112.pm9041257

56. Martinez-Rubio L, Morais S, Evensen $\varnothing$, et al. Effect of functional feeds on fatty acid and eicosanoid metabolism in liver and head kidney of Atlantic salmon (Salmo salar L.) with experimentally induced Heart and Skeletal Muscle Inflammation. Fish Shellfish Immunol. 2013;34(6):1533-1545. doi:10.1016/J.FSI.2013.03.363

57. Reddy JK, Sambasiva Rao M. Lipid Metabolism and Liver Inflammation. II. Fatty liver disease and fatty acid oxidation. Am J Physiol Liver Physiol. 2006;290(5):G852-G858. doi:10.1152/ajpgi.00521.2005

58. Narasimhan S, Gokulakrishnan K, Sampathkumar R, et al. Oxidative stress is independently associated with non-alcoholic fatty liver disease (NAFLD) in subjects with and without type 2 diabetes. Clin Biochem. 2010;43(10-11):815-821.

doi:10.1016/J.CLINBIOCHEM.2010.04.003

59. Kuroda J, Ago T, Matsushima S, Zhai P, Schneider MD, Sadoshima J. NADPH oxidase 4 (Nox4) is a major source of oxidative stress in the failing heart. Proc Natl Acad Sci U S A. 2010;107(35):15565-15570. doi:10.1073/pnas.1002178107 
60. Mas E, Danjoux M, Garcia V, Carpentier S, Ségui B, Levade T. IL-6 Deficiency Attenuates Murine Diet-Induced Non-Alcoholic Steatohepatitis. Ng IO-L, ed. PLoS One. 2009;4(11):e7929. doi:10.1371/journal.pone.0007929

61. Ix JH, Sharma K. Mechanisms linking obesity, chronic kidney disease, and fatty liver disease: the roles of fetuin-A, adiponectin, and AMPK. J Am Soc Nephrol. 2010;21(3):406-412. doi:10.1681/ASN.2009080820

62. Rossary A, Arab K, Steghens J-P. Polyunsaturated fatty acids modulate NOX 4 anion superoxide production in human fibroblasts. Biochem J. 2007;406(1):77-83. doi:10.1042/BJ20061009

63. Willcox JK, Ash SL, Catigani GL. Antioxidants and prevention of chronic disease. Crit Rev Food Sci Nutr. 2004;44(4):275-295.

64. Aykaç G, Uysal M, Süha Yalçin A, Koçak-Toker N, Sivas A, Öz H. The effect of chronic ethanol ingestion on hepatic lipid peroxide, glutathione, glutathione peroxidase and glutathione transferase in rats. Toxicology. 1985;36(1):71-76. doi:10.1016/0300-483X(85)90008-3

65. Sen CK. Glutathione homeostasis in response to exercise training and nutritional supplements. In: Stress Adaptation, Prophylaxis and Treatment. Boston, MA: Springer US; 1999:31-42. doi:10.1007/978-1-4615-5097-6_4

66. Koruk M, Taysi S, Savas MC, Yilmaz O, Akcay F, Karakok M. Oxidative stress and enzymatic antioxidant status in patients with nonalcoholic steatohepatitis. Ann Clin Lab Sci. 2004;34(1):57-62. http://www.ncbi.nlm.nih.gov/pubmed/15038668. Accessed January 28, 2019.

67. Nobili V, Pastore A, Gaeta LM, et al. Glutathione metabolism and antioxidant enzymes in patients affected by nonalcoholic steatohepatitis. Clin Chim Acta. 2005;355(1-2):105-111. doi:10.1016/J.CCCN.2004.12.022 
68. D’Archivio M, Filesi C, Varì R, Scazzocchio B, Masella R. Bioavailability of the polyphenols: status and controversies. Int J Mol Sci. 2010;11(4):1321-1342. doi:10.3390/ijms11041321

69. de Vries JH, Hollman PC, Meyboom S, et al. Plasma concentrations and urinary excretion of the antioxidant flavonols quercetin and kaempferol as biomarkers for dietary intake. Am J Clin Nutr. 1998;68(1):60-65. doi:10.1093/ajcn/68.1.60

70. Chang CY, Argo CK, Al-Osaimi AMS, Caldwell SH. Therapy of NAFLD: antioxidants and cytoprotective agents. J Clin Gastroenterol. 2006;40 Suppl 1:S51-60. doi:10.1097/01.mcg.0000168648.79034.67

71. Bobek P, Ozdín L, Hromadová M. The effect of dried tomato, grape and apple pomace on the cholesterol metabolism and antioxidative enzymatic system in rats with hypercholesterolemia. Mol Nutr Food Res. 1998;42(5):317-320. doi:10.1002/(SICI)15213803(199810)42:05<317::AID-FOOD317>3.0.CO;2-Y

72. Juśkiewicz J, Żary-Sikorska E, Zduńczyk Z, Król B, Jarosławska J, Jurgoński A. Effect of dietary supplementation with unprocessed and ethanol-extracted apple pomaces on caecal fermentation, antioxidant and blood biomarkers in rats. Br J Nutr. 2012;107(8):1138-1146. doi:10.1017/S0007114511004144

73. Lyngdoh T, Marques-Vidal P, Paccaud F, et al. Elevated Serum Uric Acid Is Associated with High Circulating Inflammatory Cytokines in the Population-Based Colaus Study. Means T, ed. PLoS One. 2011;6(5):e19901. doi:10.1371/journal.pone.0019901

74. Hyson DA. A comprehensive review of apples and apple components and their relationship to human health. Adv Nutr. 2011;2(5):408-420. doi:10.3945/an.111.000513 
75. Devi PB, Vijayabharathi R, Sathyabama S, Malleshi NG, Priyadarisini VB. Health benefits of finger millet (Eleusine coracana L.) polyphenols and dietary fiber: a review. J Food Sci Technol. 2014;51(6):1021-1040. doi:10.1007/s13197-011-0584-9

76. Saura-Calixto F. Antioxidant Dietary Fiber Product: A New Concept and a Potential Food Ingredient. 1998. doi:10.1021/JF9803841

77. Sudha ML, Baskaran V, Leelavathi K. Apple pomace as a source of dietary fiber and polyphenols and its effect on the rheological characteristics and cake making. Food Chem. 2007;104(2):686-692. doi:1016/J.FOODCHEM.2006.12.016

78. Sembries S, Dongowski G, Jacobasch G, Mehrländer K, Will F, Dietrich H. Effects of dietary fibre-rich juice colloids from apple pomace extraction juices on intestinal fermentation products and microbiota in rats. Br J Nutr. 2003;90(3):607-615. doi:10.1079/BJN2003925

79. Sembries S, Dongowski G, Mehrländer K, Will F, Dietrich H. Dietary fiber-rich colloids from apple pomace extraction juices do not affect food intake and blood serum lipid levels, but enhance fecal excretion of steroids in rats. J Nutr Biochem. 2004;15(5):296-302. doi:10.1016/J.JNUTBIO.2003.12.005

80. Chen L, Liu L, Li C, et al. A mix of apple pomace polysaccharide improves mitochondrial function and reduces oxidative stress in the liver of high-fat diet-induced obese mice. Mol Nutr Food Res. 2017;61(3). doi:10.1002/mnfr.201600433 
Table 1. Fatty acid analysis of rodent diets substituted with apple pomace $(10 \% \mathrm{~g} / \mathrm{kg})$.

\begin{tabular}{lccccc}
\hline \multirow{2}{*}{ Measurements (mg/g) } & \multicolumn{5}{c}{ Treatments } \\
\cline { 2 - 6 } & AIN & AIN/AP & Western & Western/AP & $\boldsymbol{p}$-Value \\
\hline SFAs & & & & & \\
Palmitic acid (16:0) & $113.6 \pm 0.9^{\mathrm{c}}$ & $111.4 \pm 1.5^{\mathrm{c}}$ & $321.9 \pm 0.3^{\mathrm{b}}$ & $329.2 \pm 3.0^{\mathrm{a}}$ & $<0.0001$ \\
Stearic acid (18:0) & $35.6 \pm 2.5^{\mathrm{b}}$ & $37.2 \pm 0.6^{\mathrm{b}}$ & $99.4 \pm 1.1^{\mathrm{a}}$ & $102.4 \pm 0.6^{\mathrm{a}}$ & $<0.0001$ \\
\hline MUFAs & & & & & \\
Palmitoleic acid (16:1n-7) & $0 \pm 0.00^{\mathrm{b}}$ & $0 \pm 0.00^{\mathrm{b}}$ & $14.4 \pm 0.1^{\mathrm{a}}$ & $14.4 \pm 0.2^{\mathrm{a}}$ & $<0.0001$ \\
Oleic acid (18:1n-9) & $190.9 \pm 1.0^{\mathrm{b}}$ & $183.5 \pm 3.3^{\mathrm{b}}$ & $229.6 \pm 1.1^{\mathrm{a}}$ & $229.5 \pm 1.7^{\mathrm{a}}$ & $<0.0001$ \\
\hline PUFAs & & & & & \\
Linoleic acid (18:2 n-6) & $501.2 \pm 5.5^{\mathrm{a}}$ & $514.1 \pm 24.1^{\mathrm{a}}$ & $69.9 \pm 0.9^{\mathrm{b}}$ & $70.4 \pm 0.6^{\mathrm{b}}$ & $<0.0001$ \\
a-linolenic acid (18:3 n-3) & $70.8 \pm 1.3^{\mathrm{a}}$ & $71.3 \pm 7.0^{\mathrm{a}}$ & $10.4 \pm 0.1^{\mathrm{b}}$ & $10.5 \pm 0.2^{\mathrm{b}}$ & $<0.0001$ \\
Arachidonic acid (20:4 n-6) & $0 \pm 0.00^{\mathrm{b}}$ & $0 \pm 0.00^{\mathrm{b}}$ & $0.13 \pm 0.00^{\mathrm{a}}$ & $0.14 \pm 0.00^{\mathrm{a}}$ & $<0.0001$ \\
EPA (20:5n-3) & $0 \pm 0.00$ & $0 \pm 0.00$ & $0 \pm 0.00$ & $0 \pm 0.00$ & 1.00 \\
DHA (22:6n-3) & $0 \pm 0.00$ & $0 \pm 0.00$ & $0 \pm 0.00$ & $0 \pm 0.00$ & 1.00 \\
\hline
\end{tabular}

Values expressed as mean \pm standard error of the mean (SEM, $n=5$ samples/group). Different superscript letters $a$, $b$, and $c$ within. The same row indicates significant difference at $p \leq 0.05$ by one-way ANOVA followed by Tukey's test. Abbreviations: DHA, docosahexaenoic acid; EPA, eicosapentaenoic acid; MUFAs, monounsaturated fatty acids; PUFAs, polyunsaturated fatty, acids; SFAs, saturated fatty acids. 
Table 2. Daily caloric and macronutrient intake, total body weight gain, liver and gonadal fat pad weights, and liver triglyceride content of growing female rats consuming different diets substituted with apple pomace $(10 \% \mathrm{~g} / \mathrm{kg})$ for 8 weeks.

\begin{tabular}{|c|c|c|c|c|c|}
\hline \multirow{2}{*}{ Measurements } & \multicolumn{5}{|c|}{ Treatments } \\
\hline & AIN & AIN/AP & Western & Western/AP & $p$-Value \\
\hline Caloric intake (kcal/d) & $53 \pm 2^{b}$ & $49 \pm 1^{b}$ & $60 \pm 1^{a}$ & $61 \pm 2^{a}$ & $<0.0001$ \\
\hline $\mathrm{CHO}$ intake (kcal/d) & $32 \pm 1^{a}$ & $31 \pm 1^{a}$ & $24 \pm 1^{b}$ & $24 \pm 1^{b}$ & $<0.0001$ \\
\hline Fat intake(kcal/d) & $9 \pm 0.3^{b}$ & $8 \pm 0.2^{b}$ & $27 \pm 1^{a}$ & $27 \pm 1^{a}$ & $<0.0001$ \\
\hline Bwt gain (g/d) & $2.2 \pm 0.1$ & $2.2 \pm 0.1$ & $2.4 \pm 0.1$ & $2.5 \pm 0.1$ & 0.17 \\
\hline Final Body Weight (g) & $215 \pm 4$ & $216 \pm 8$ & $229 \pm 5$ & $234 \pm 5$ & 0.08 \\
\hline Gonadal fat pad weight $(\mathrm{g})$ & $4.12 \pm 0.26^{b}$ & $3.46 \pm 0.44^{\mathrm{b}}$ & $5.87 \pm 0.24^{a}$ & $5.96 \pm 0.23^{a}$ & $<0.0001$ \\
\hline Relative gonadal fat pad weight (mg/g bwt) & $1.90 \pm 0.14^{\mathrm{b}}$ & $1.59 \pm 0.17^{b}$ & $2.37 \pm 0.20^{a}$ & $2.56 \pm 0.11^{a}$ & $<0.0001$ \\
\hline Liver weight $(\mathrm{g})$ & $7.50 \pm 0.24$ & $7.44 \pm 0.37$ & $8.05 \pm 0.30$ & $7.98 \pm 0.24$ & 0.35 \\
\hline Relative liver weight (mg/g bwt) & $3.47 \pm 0.08$ & $3.45 \pm 0.07$ & $3.52 \pm 0.07$ & $3.41 \pm 0.05$ & 0.69 \\
\hline
\end{tabular}

Values expressed as mean \pm SEM ( $n=6-8$ rats/group). Different superscript letters $a$ and $b$ within the same row. Indicate significant difference at $p \leq 0.05$ by one-way ANOVA followed by Tukey's test. Abbreviations: Bwt, body weight; CHO, carbohydrate. 
Table 3. Liver and gonadal adipose tissue fatty acid content of young female rats consuming different diets substituted with apple pomace $(10 \% \mathrm{~g} / \mathrm{kg})$.

\begin{tabular}{|c|c|c|c|c|c|c|c|c|c|c|}
\hline \multicolumn{5}{|c|}{ Liver } & \multicolumn{6}{|c|}{ Adipose } \\
\hline $\begin{array}{l}\text { Measurement } \\
(\mathrm{mg} / \mathrm{g})\end{array}$ & AIN & AIN/AP & Western & Western/AP & $p$-value & AIN & AIN/AP & Western & Western/AP & $p$-value \\
\hline \multicolumn{11}{|l|}{ SFAs } \\
\hline $\begin{array}{l}\text { Palmitic Acid } \\
(16: 0)\end{array}$ & $191.0 \pm 5.7^{\mathrm{bc}}$ & $184.0 \pm 5.33^{c}$ & $214.4 \pm 5.3^{a}$ & $210.8 \pm 5.3^{\mathrm{ab}}$ & 0.0007 & $132.1 \pm 27.3^{b}$ & $282.6 \pm 25.6^{a}$ & $123.2 \pm 29.5^{b}$ & $241.8 \pm 25.6^{a}$ & 0.0004 \\
\hline $\begin{array}{l}\text { Stearic Acid } \\
(18: 0)\end{array}$ & $144.6 \pm 6.5$ & $140.6 \pm 6.0$ & $148.3 \pm 8.3$ & $162.8 \pm 6.0$ & 0.08 & $18.3 \pm 3.1^{\mathrm{b}}$ & $32.9 \pm 3.3^{a}$ & $20.2 \pm 3.10^{\mathrm{b}}$ & $35.5 \pm 3.1^{\mathrm{a}}$ & 0.0008 \\
\hline \multicolumn{11}{|l|}{ MUFAs } \\
\hline $\begin{array}{l}\text { Palmitoleic } \\
\text { Acid (16:1) }\end{array}$ & $57.0 \pm 25.0^{b}$ & $81.0 \pm 25.0^{b}$ & $161.0 \pm 23.0^{\mathrm{a}}$ & $76.0 \pm 25.0^{b}$ & 0.05 & $14.7 \pm 7.3$ & $24.2 \pm 10.4$ & $17.8 \pm 7.0$ & $23.6 \pm 8.8$ & 0.84 \\
\hline $\begin{array}{l}\text { Oleic Acid } \\
(18: 1)\end{array}$ & $112.5 \pm 16.5^{\mathrm{b}}$ & $107.2 \pm 13.9^{b}$ & $195.5 \pm 13.9^{a}$ & $159.5 \pm 13.0^{\mathrm{ab}}$ & 0.0005 & $185.8 \pm 39.9^{b}$ & $313.7 \pm 39.9^{a}$ & $142.4 \pm 46.1^{b}$ & $314.9 \pm 42.6^{a}$ & 0.01 \\
\hline \multicolumn{11}{|l|}{ PUFAs } \\
\hline LA (18:3n-6) & $224.4 \pm 10.9^{a}$ & $251.2 \pm 10.9^{a}$ & $92.8 \pm 10.9^{b}$ & $83.8 \pm 10.9^{b}$ & $<0.0001$ & $193.8 \pm 65.0^{\mathrm{ab}}$ & $376.1 \pm 51.0^{\mathrm{a}}$ & $53.5 \pm 19.0^{b}$ & $64.6 \pm 6.7^{b}$ & $<0.0001$ \\
\hline ALA (18:3n-3) & $10.4 \pm 1.4^{\mathrm{a}}$ & $12.8 \pm 1.5^{\mathrm{a}}$ & $2.3 \pm 1.4^{b}$ & $2.2 \pm 1.4^{b}$ & $<0.0001$ & $11.3 \pm 3.5^{\mathrm{b}}$ & $38.4 \pm 5.0^{\mathrm{a}}$ & $3.8 \pm 2.2^{b}$ & $5.2 \pm 1.6^{b}$ & $<0.0001$ \\
\hline ARA (20:4) & $132.6 \pm 10.0$ & $119.9 \pm 16.1$ & $132.0 \pm 10.0$ & $145.6 \pm 10.0$ & 0.37 & $0 \pm 0.00$ & $0 \pm 0.00$ & $0 \pm 0.00$ & $0 \pm 0.00$ & 1.00 \\
\hline EPA $(20: 5 n-3)$ & $0 \pm 0.00$ & $0 \pm 0.00$ & $0 \pm 0.00$ & $0 \pm 0.00$ & 1.00 & $0 \pm 0.00$ & $0 \pm 0.00$ & $0 \pm 0.00$ & $0 \pm 0.00$ & 1.00 \\
\hline DHA (22:6n-3) & $0 \pm 0.00$ & $0 \pm 0.00$ & $0 \pm 0.00$ & $0 \pm 0.00$ & 1.00 & $0.1 \pm 0.1^{b}$ & $1.3 \pm 0.3^{a}$ & $0 \pm 0.00^{b}$ & $0.1 \pm 0.1^{b}$ & 0.0078 \\
\hline
\end{tabular}

Values expressed as mean \pm SEM ( $n=6-8$ rats/group). Different superscript letters $a$ and $b$ within the same column indicate significant difference at $p \leq 0.05$ by one-way ANOVA followed by Tukey's test. Abbreviations: ALA, $\alpha$-linolenic acid; ARA, arachidonic acid; DHA, docosahexaenoic acid; EPA, eicosapentaenoic acid; LA, linoleic acid; MUFA, monounsaturated fatty acids; PUFA, polyunsaturated fatty acids; SFAs, saturated fatty acids 
Table 4. Effect of consumption of different diets substituted with apple pomace $(10 \% \mathrm{~g} / \mathrm{kg})$ by growing female rats on serum and urine measurements of liver function enzymes, and uric acid following 8 weeks of feeding.

\begin{tabular}{lccccc}
\hline \multicolumn{1}{c}{ Measurements } & AIN & AIN/AP & Western & Western/AP & $p$-Value \\
\hline Serum ALT $(\mathrm{U} / \mathrm{L})$ & $107.63 \pm 19.59$ & $118.71 \pm 43.60$ & $94.5 \pm 12.58$ & $133.5 \pm 30.59$ & 0.78 \\
Serum AST $(\mathrm{U} / \mathrm{L})$ & $129.48 \pm 52.86$ & $212.50 \pm 37.86$ & $283.63 \pm 45.30$ & $259.67 \pm 48.96$ & 0.69 \\
Serum AST:ALT ratio & $3.16 \pm 0.45$ & $2.79 \pm 0.28$ & $2.97 \pm 0.26$ & $2.84 \pm 0.17$ & 0.83 \\
Serum Bilirubin & $0.21 \pm 0.01$ & $0.21 \pm 0.01$ & $0.21 \pm 0.01$ & $0.21 \pm 0.01$ & 1.00 \\
Serum Albumin & $4.06 \pm 0.24$ & $4.28 \pm 0.36$ & $4.93 \pm 0.21$ & $4.1 \pm 0.36$ & 0.21 \\
Serum Uric Acid $(\mu \mathrm{M})$ & $7.24 \pm 0.31$ & $6.27 \pm 1.61$ & $7.19 \pm 0.86$ & $7.57 \pm 1.25$ & 0.86 \\
Urine Uric Acid $(\mu \mathrm{M})$ & $5.94 \pm 2.26$ & $10.35 \pm 2.11$ & $10.40 \pm 1.12$ & $6.79 \pm 1.41$ & 0.23 \\
\hline
\end{tabular}

Values expressed as mean \pm SEM ( $n=4-8$ animals/group). Different superscript letters $a$ and $b$ within the same figure indicates significant difference at $p \leq 0.05$ by one-way ANOVA followed by Tukey's test. Abbreviations: AST, aspartate aminotransferase; ALT, alanine aminotransferase. 


\section{Figure Legend}

Figure 1. Representative histological staining images of the liver of growing female rats consuming (A) AIN, (B) AIN/AP, (C) Western, or (D) Western/AP following 8 weeks of feeding. Black arrows indicate fat deposition. White arrows indicate inflammation.

Figure 2. Relative expression of genes involved in inflammation in (A) liver tissue and (B) gonadal adipose tissue of young female rats consuming different diets substituted with apple pomace $(10 \% \mathrm{~g} / \mathrm{kg})$ for 8 weeks. Values expressed as mean \pm SEM ( $\mathrm{n}=6-8$ animals/group). Different superscript letters $a$ and $b$ within the same figure indicates significant difference at $p \leq 0.05$ by one-way ANOVA followed by Tukey's test. Abbreviations: AU, arbitrary units, IL- 6 , interleukin-6, IL-10, interleukin-10; NFKB, nuclear factor kappa-light-chain enhancer of activated B cells; TNF- $\alpha$, tumor necrosis factor alpha.

Figure 3. Relative expression of genes involved in polyunsaturated fatty acid metabolism, inflammation, and oxidative stress in (A) liver tissue and (B) gonadal adipose tissue of young female rats consuming different diets substituted with apple pomace $(10 \% \mathrm{~g} / \mathrm{kg})$ for 8 weeks. Values expressed as mean \pm SEM ( $n=6-8$ animals/group). Different superscript letters a and $b$ within the same figure indicates significant difference at $p \leq 0.05$ by one-way ANOVA followed by Tukey's test. Abbreviations: 5LOX, arachidonate 5-lipoxygenase; AU, arbitrary units; COX1, cyclooxygenase 1; COX2, cyclooxygenase 2; NOX4, NADPH oxidase 4.

Figure 4. Relative expression of genes involved in oxidative stress and antioxidant function in liver tissue of young female rats consuming different diets substituted with apple pomace (10\% $\mathrm{g} / \mathrm{kg}$ ) for 8 weeks. Values expressed as mean \pm SEM ( $\mathrm{n}=6-8$ animals/group). Different superscript letters $a$ and $b$ within the same figure indicates significant difference at $p \leq 0.05$ by one-way ANOVA followed by Tukey's test. Abbreviations: AU, arbitrary units; CAT, catalase; CCL-2, chemokine (C-C motif) ligand 2; GPx, glutathione peroxidase; Nrf2, nuclear factor-like 2; 
SOD1, superoxide dismutase 1 ; SOD2, superoxide dismutase 2 , TGF $\beta 3$, transforming growth factor beta-3.

Figure 5. Oxidative stress and antioxidant status measured by (A) hepatic hydrogen peroxide, (B) hepatic MDA, (C) hepatic total polyphenols, (D) serum total antioxidants, and (E) urine total antioxidants in young female rats consuming different diets substituted with apple pomace (10\% $\mathrm{g} / \mathrm{kg}$ ) for 8 weeks. Values expressed as mean \pm SEM ( $n=6-8$ animals/group). Different superscript letters $a$ and $b$ within the same panel indicates significant difference at $p \leq 0.05$ by one-way ANOVA followed by Tukey's test. Abbreviations: MDA, malondialdehyde. 


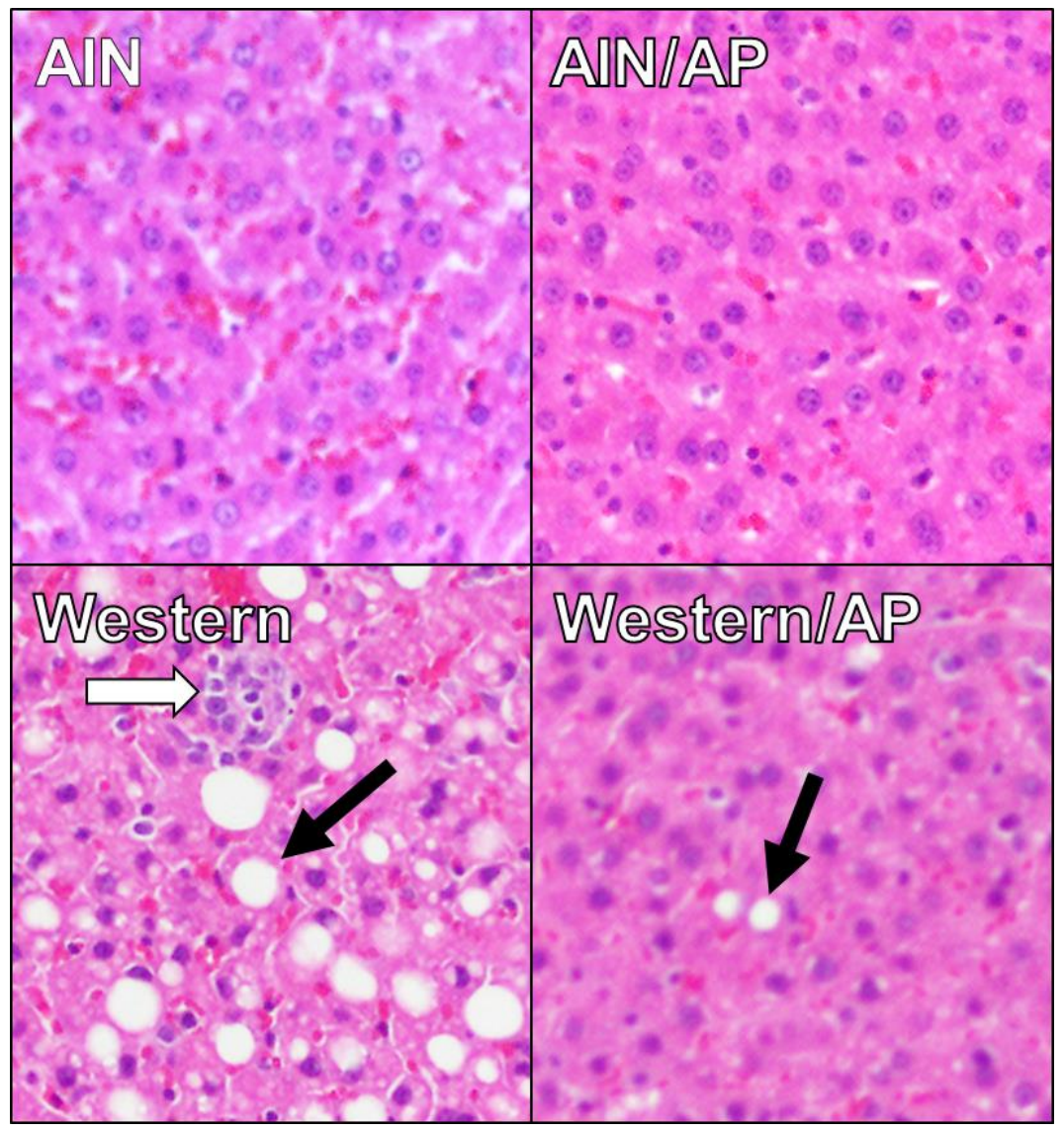

\begin{tabular}{lcccc}
\hline Inflammation Grades & & & & \\
& AIN & AIN/AP & Western & Western/AP \\
\hline 0 & $85 \%$ & $85 \%$ & $12 \%$ & $37 \%$ \\
1 & $15 \%$ & $15 \%$ & $88 \%$ & $63 \%$ \\
\hline
\end{tabular}



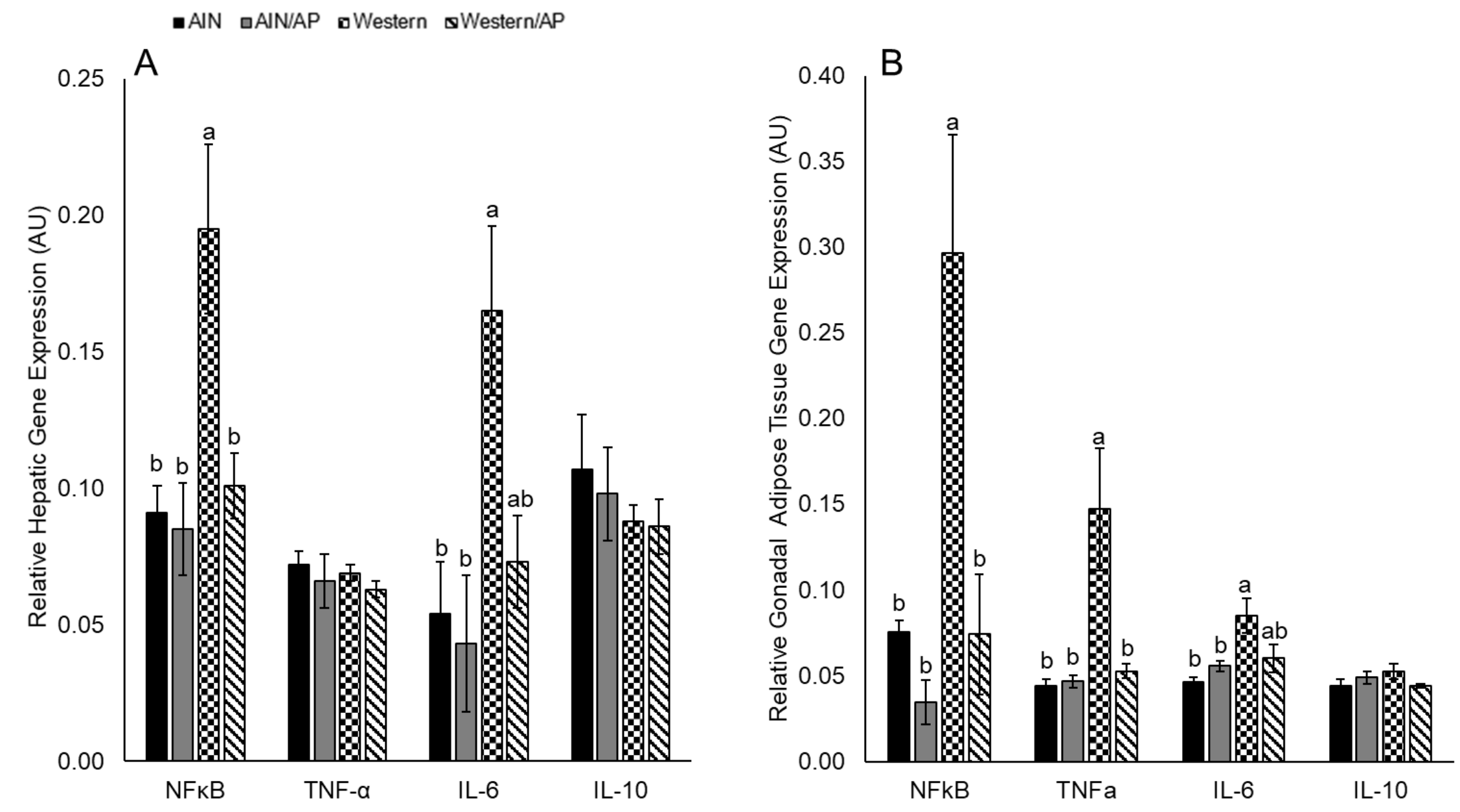



153 


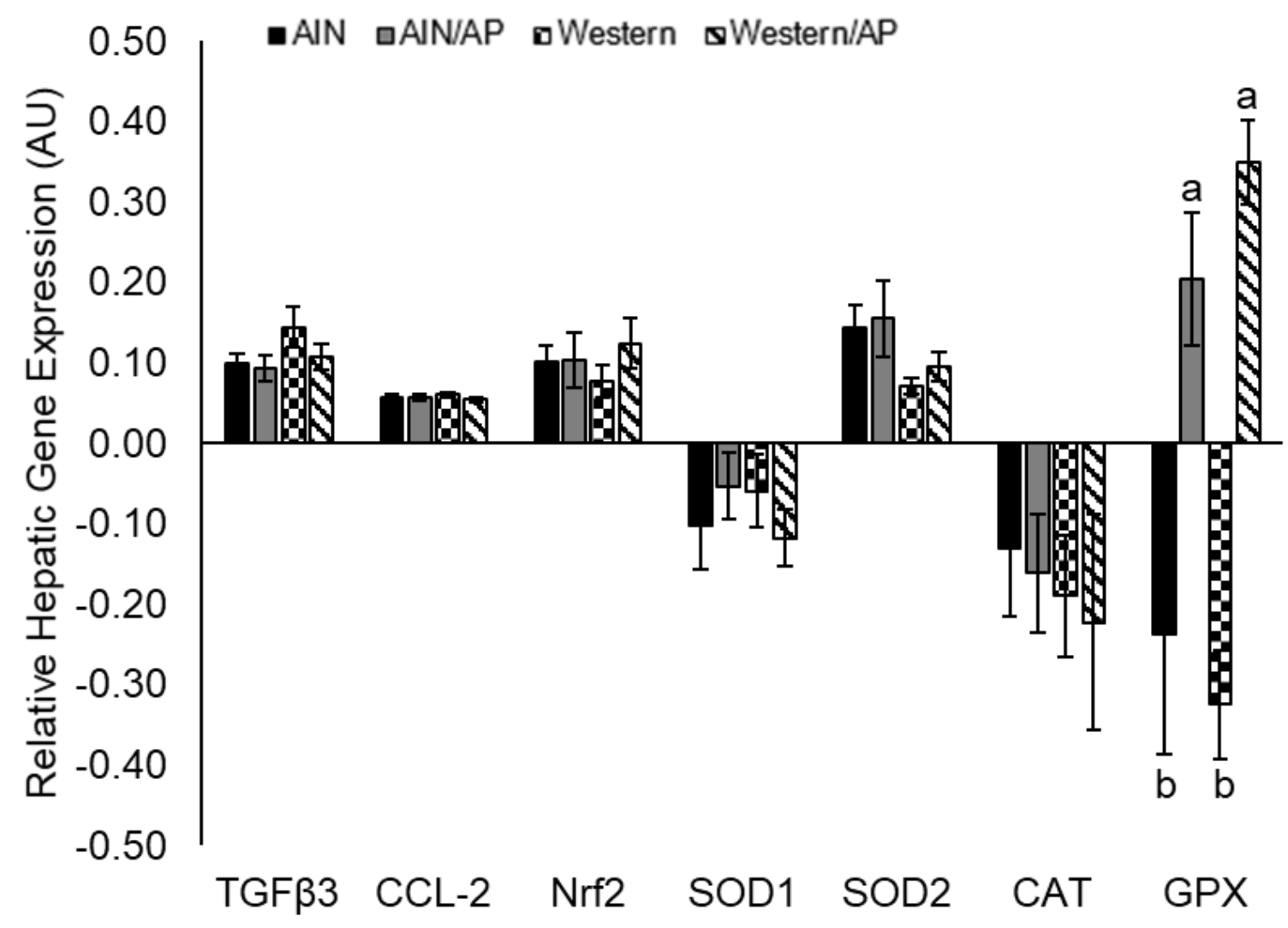



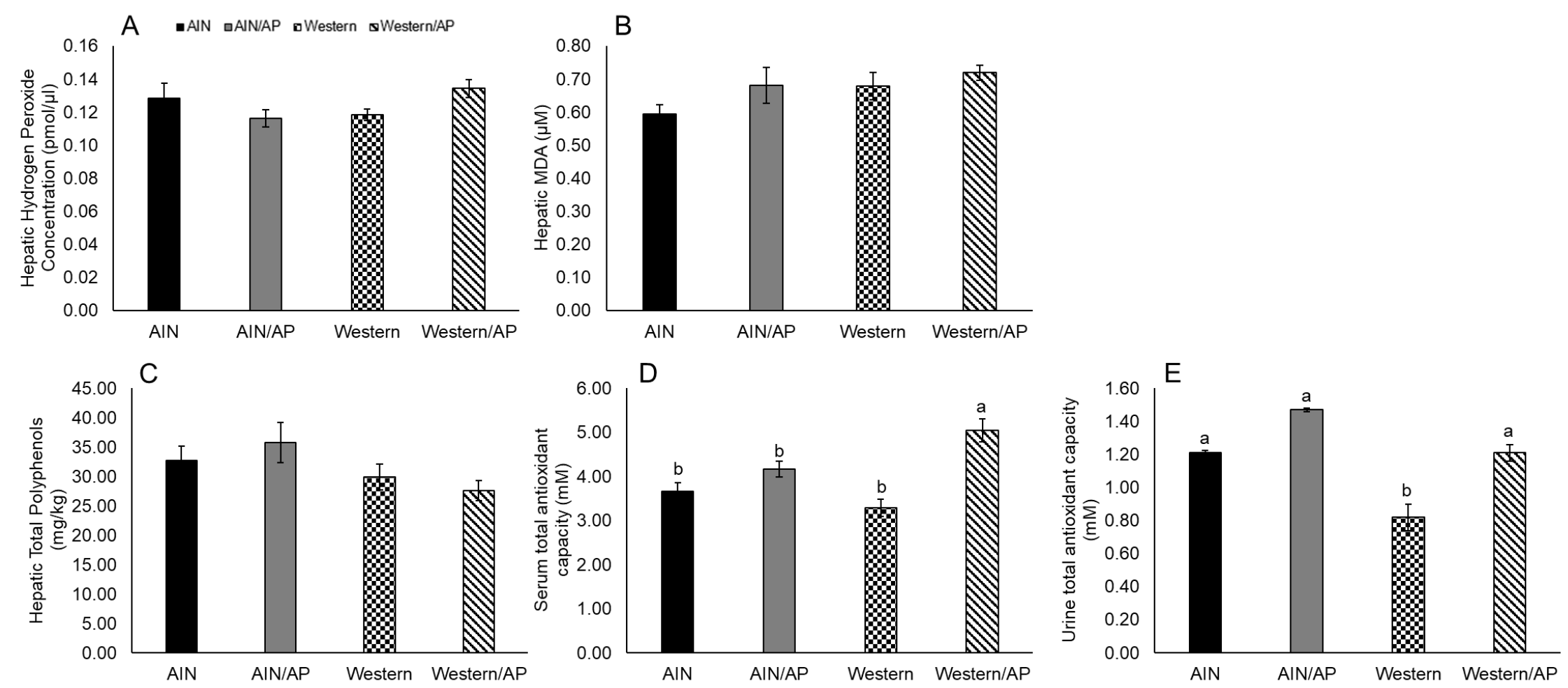


\subsection{Supplementary Material}

Supplementary Table 1 . Composition of rodent diets substituted with apple pomace $(10 \% \mathrm{~g} / \mathrm{kg})$ fed to growing female rats.

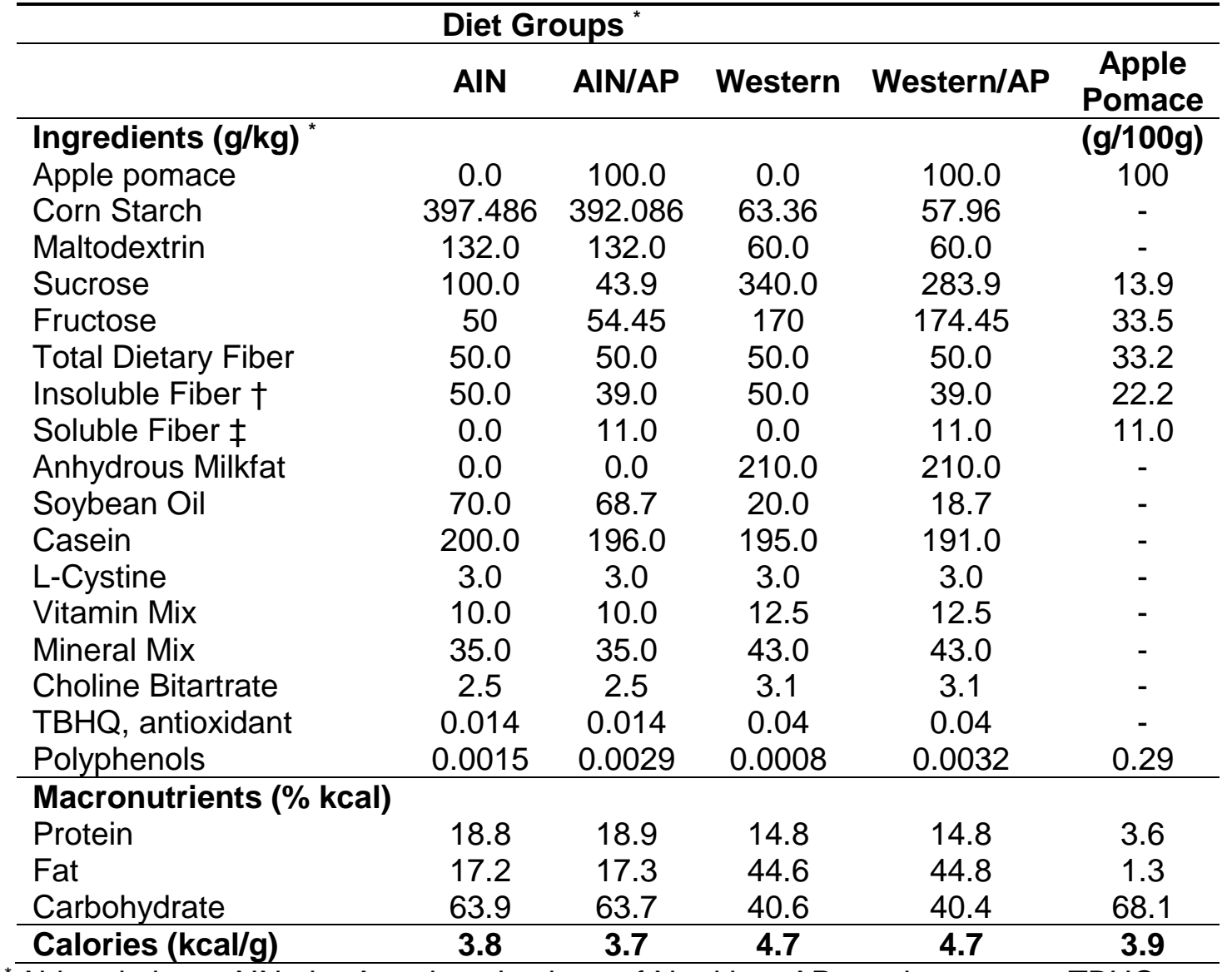

* Abbreviations: AIN, the American Institute of Nutrition; AP, apple pomace; TBHQ, tertbutylhydroquinone. $\dagger$ Insoluble fiber is cellulose. $\ddagger$ Soluble fiber is mainly pectin ${ }^{2}$. 
Supplementary Table 2. List of primers used for RT-qPCR analysis.

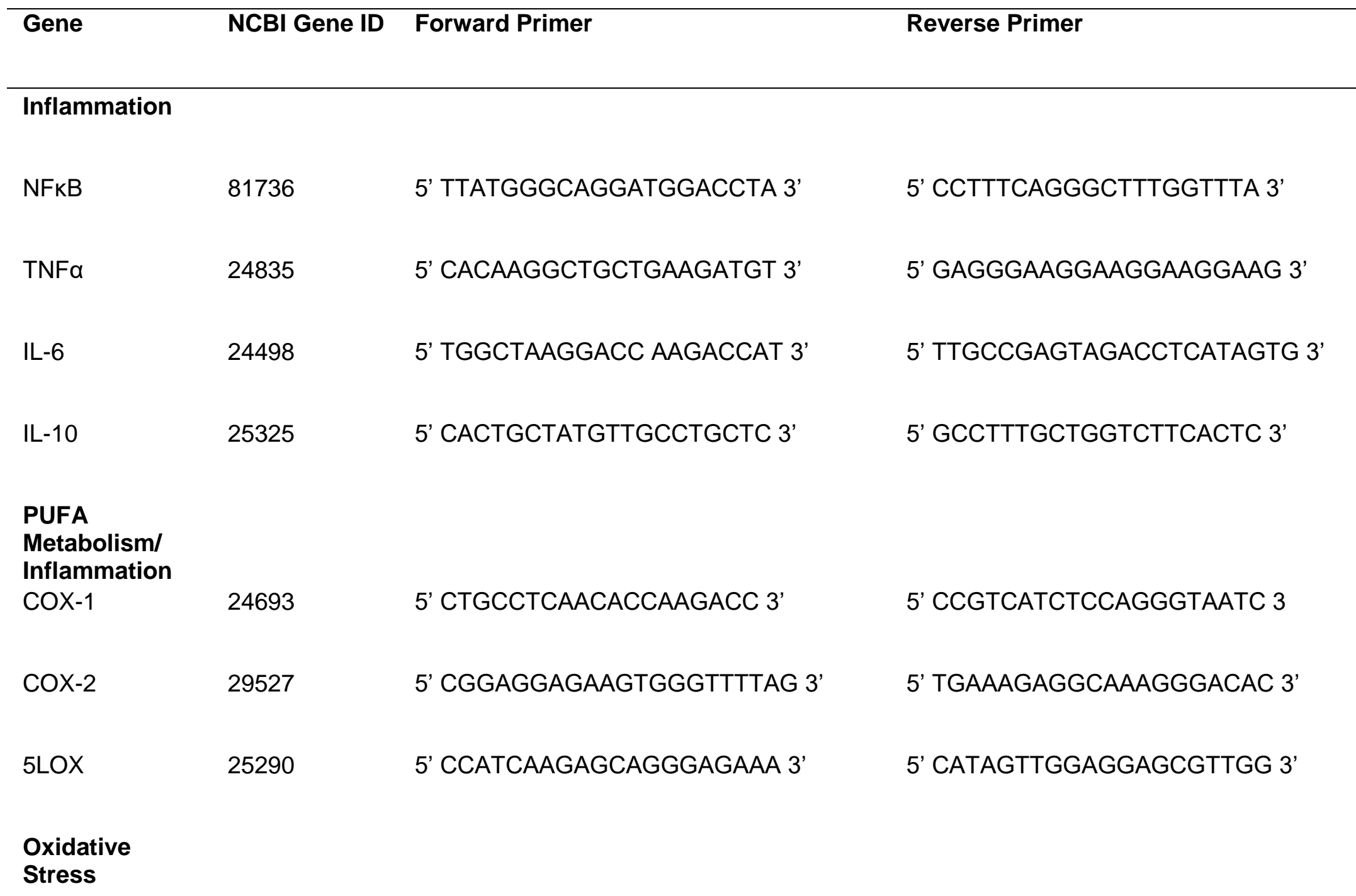




\begin{tabular}{|c|c|c|c|}
\hline NOX4 & 85431 & 5’ CСТCCATCAAGCCAAGATTC 3' & 5' CTCCAGCCACACACAGACTAAC 3' \\
\hline TGFß3 & 25717 & 5' AACTGCTGTGTGCGCCCC 3' & 5' TAGTCCAAGCACCGTGCTGTGG 3' \\
\hline CCL-2 & 24770 & 5' CCACAACCACCTCAAGCAC 3' & 5' AGGCATCACATTCCAAATCAC 3' \\
\hline \multicolumn{4}{|c|}{$\begin{array}{l}\text { Antioxidant } \\
\text { Status }\end{array}$} \\
\hline Nrf2 & 83619 & 5' TGACTCGGAAATGGAAGAGC 3' & 5' TGTGTTGGCTGTGCTTTAGG 3' \\
\hline SOD1 & 24786 & 5' GGTCCACGAGAAACAAG TGA 3' & 5' CAATCACACCACAAGCCAAG 3' \\
\hline SOD2 & 24787 & 5' GAAAGTGCTCAAGATGGACAAAG 3' & 5' CTGAATGGCTTCCCTGAATG 3' \\
\hline Catalase & 24248 & 5' TGTTGAATGAGGAGAGGA 3' & 5' TTCTTAGGC TTCTGGGAGTTG 3' \\
\hline GPx & 24404 & 5' GATACGCCGAGTGTGGTT T 3' & 5' TCTTGATTACTTCCTGGCTCCT 3' \\
\hline \multicolumn{4}{|c|}{ Housekeeping } \\
\hline$\beta$-actin & 81822 & 5' TTGCTGACAGGATGCACAAG 3' & 5' CAGTGAGGCCAGGATAGAGC 3' \\
\hline GAPDH & 24383 & 5' TCAAGAAGGTGGTGAAGCAG 3' & 5' CCTCAGTGTAGCCCAGGATG 3' \\
\hline
\end{tabular}


Caloric Substitution of Diets with Apple Pomace was Determined to be Safe for Renal and Bone Health Using a Growing Rat Model

R. Chris Skinner ${ }^{1}$, Joseph C. Gigliotti ${ }^{2}$, Katherine H. Taylor ${ }^{1}$, Derek C. Warren ${ }^{1}$, Vagner A. Benedito $^{3}$, Janet C. Tou ${ }^{1 *}$

${ }^{1}$ Division of Animal and Nutritional Sciences, ${ }^{2}$ Department of Integrative Physiology and

Pharmacology, Liberty University College of Osteopathic Medicine, Liberty, VA, 24515, United

States, ${ }^{3}$ Division of Plant and Soil Sciences, West Virginia University, Morgantown, WV 26506

Corresponding Author:

Janet C. Tou, PhD

Division of Animal and Nutritional Sciences

West Virginia University

Morgantown, WV 26506

Tel: (304)293-1919

Fax: (304)293-2232

e-mail: janet.tou@mail.wvu.edu 


\subsection{Abstract}

Apple pomace, a "waste" byproduct of apple processing has a favorable nutritional profile indicating potential for repurposing for human consumption. However, the high fructose content of apple pomace when added to a healthy diet or Western diet, typified by high sugar and high fat, may result in detriments to kidney and bone health. Therefore, the objectives of this study were to determine the safety of caloric substitution with $10 \%$ apple pomace substitution $(\mathrm{g} / \mathrm{kg})$ to a healthy or Western diet. Growing (age 22-29 days) female Sprague-Dawley rats were randomly assigned ( $n=8$ rats/group) to consume a purified standard rodent diet (AIN-93G), AIN93G/10\% g/kg apple pomace (AIN/AP), Western diet, or Western/10\% g/kg apple pomace (Western/AP) diets for 8 weeks. Histological evaluation showed renal interstitial hypercellularity in rats fed AIN/AP, Western, and Western/AP diets. However, there was no effects on renal expression of oxidative stress and inflammatory genes or serum measures of kidney damage and function among diet groups. Apple pomace is also high in calcium which can affect calcium balance. Dietary calcium consumption was highest $(p<0.0001)$ in rats consuming Western/AP. However, there was no significant differences in calcium absorption and retention among diet groups. Further, there was no evidence of renal calcification. There were also impact on femoral calcium and total mineral content, size, and strength. Based on the results, apple pomace consumption was safe for renal and bone health, regardless of diet quality. Keywords: apple pomace, safety, minerals, Western diet, bone, kidney 


\subsection{Introduction}

Apple processing generates waste, consisting of skin, stem, seeds, and calyx,

collectively known as apple pomace. The environmental pollution and burden of waste disposal costs to apple farmers and producers can be decreased by re-purposing apple pomace as a product for human consumption [1-3]. However, among popular consumed fruits, apples had the highest fructose content [1]. Muir, et al. [4] reported apples to have $10.5 \mathrm{~g}$ of fructose/serving compared to $3.2 \mathrm{~g} / \mathrm{serving}$ for bananas, $6.4 \mathrm{~g} / \mathrm{serving}$ for blueberries, and $2.5 \mathrm{~g} / \mathrm{serving}$ for oranges. Further, apple pomace contains $44.7 \%$ fructose compared to $5.8-6.0 \%$ fructose in whole apple [5]. This is a health concern because fructose overconsumption has been reported to contribute to renal disease and to produce deleterious effects on bone [6,7]. Apple pomace contains a higher mineral content than whole apples, particularly calcium which is required for bone health $[1,8]$. However, over-consumption of calcium can increase nephrocalcinosis and reduced kidney function $[9,10]$. In turn, renal dysfunction can lead to bone loss due to mineral imbalance, resulting in increased risk of osteoporosis and other bone-mineral disorders [11].

Diets typical of Western countries are characterized by high fat and high sucrose. Western diet consumption has been shown to increase the risk of chronic kidney disease by inducing renal steatosis, inflammation, and oxidative stress. Western diet consumption has also been reported to increase risk of kidney stones due to the high sugar content $[12,13]$. Additionally, consuming a Western diet can result in early onset of osteoporosis by promoting mineral balance and inflammation leading to decreased bone mineral density $[14,15]$. Dietary advice suggests replacing calories in the diet with healthier food choices instead of dietary supplementation with a purified isolated nutrient [16].

Previously, our laboratory reported caloric substitution of a Western diet with $10 \% \mathrm{~g} / \mathrm{kg}$ apple pomace attenuating features of NAFLD [17]. However, the effects of apple pomace on renal and bone was not assessed in this study. To our knowledge no studies have evaluated the safety of apple pomace consumption on renal and bone health. Therefore, the objectives of this 
study were to determine the safety of apple pomace, due to its high fructose content and increased calcium content, in growing rats consuming a standard diet or Western diet. Female rats were used due to their increased susceptibility to nephrocalcinosis, and growing rats because kidney disease has been shown to have more severe bone effects in a pediatric population $[18,19]$. We hypothesize apple pomace will not have detriment to kidney or bone health in growing female rats consuming "healthy" or Western diets.

\subsection{Materials and Methods}

Diets

Locally sourced apple pomace was provided by Swilled Dog Hard Cider Company (Franklin, WV). Apple pomace was freeze dried in a VirTis Genesis 25 XL Pilot Freeze Drier (SP Scientific, Warminster, PA) Nutrient composition analysis of apple pomace was performed by Medallion Laboratories (Minneapolis, MN). Apple pomace contains 32.5\% fructose compared to the published average of $5.9 \%$ fructose for whole apples. Dietary calcium and phosphorus were determined by inductively coupled plasma mass spectrometry (ICP) (model P400, Perkin Elmer, Shelton, CT). Apple pomace contained $1.47 \mathrm{mg} / \mathrm{g}$ calcium and $1.97 \mathrm{mg} / \mathrm{g}$ phosphorous compared to published values of $0.06 \mathrm{mg} / \mathrm{g}$ calcium and $0.11 \mathrm{mg} / \mathrm{g}$ phosphorous in whole apples [17] (Table 1).

The 'healthy' diet was the standard purified American Institute of Nutrition (AIN-93G) for growing rats [20] while a Western diet consisting of $45 \%$ fat and $34 \%$ sucrose was used to typify the high fat, high sugar diet consumed by Western countries [21,22]. AIN-93G and Western diet were calorically substituted with $10 \% \mathrm{~g} / \mathrm{kg}$ freeze-dried apple pomace. AIN diets were adjusted to be isocaloric (3.7-3.8 kcal/g) and Western diets were adjusted to be isocaloric $(4.7 \mathrm{kcal} / \mathrm{g})$. Table 2 shows diet formulation for macronutrients, sugars, total minerals, calcium, and phosphorous. The complete ingredient composition of experimental diets is provided in Supplemental Table 1. Diets were stored at $-20^{\circ} \mathrm{C}$ until fed to animals. 
Animals

Weanling (age 22-29 days) female Sprague-Dawley rats $(n=32)$ were purchased from Harlan-Tekald (Indianapolis, IN). All animal procedures were approved by the Animal Care and Use Committee at West Virginia University and conducted in accordance with the guidelines of the National Research Council for the Care and Use of Laboratory Animals [23]. Rats were individually housed and kept in a room at constant temperature of $21+2^{\circ} \mathrm{C}$ with a $12 \mathrm{~h}$ light/dark cycle throughout the study duration. Following 7-days acclimation period, rats were randomly assigned ( $n=8$ rats/group) to four dietary groups consisting of: 1) AIN-93G, 2) AIN-93G with $10 \%$ weight $(\mathrm{g} / \mathrm{kg})$ substituted with apple pomace (AIN/AP), 3) Western diet (45\% fat, 33\% sucrose by kcals), or 4) Western diet with $10 \%$ of weight $(\mathrm{g} / \mathrm{kg})$ substituted with apple pomace (Western/AP). Rats were provided ad libitum access to their assigned diets and deionized distilled water $\left(\mathrm{ddH}_{2} \mathrm{O}\right)$ throughout the eight weeks study duration. Food intake was measured and assigned diets replaced every other day while ddH2O was replaced weekly. At the end of the study, rats were fasted overnight then euthanized by carbon dioxide inhalation. The kidney was excised, weighed, and then flash frozen in liquid nitrogen and stored at $-80^{\circ} \mathrm{C}$ until analyzed. Both femurs were removed, cleaned, and stored at $-20^{\circ} \mathrm{C}$.

Kidney histology

The left kidney was removed, weighed, flash frozen in liquid nitrogen, and stored at $80^{\circ} \mathrm{C}$ until analysis. A center sagittal section was cut from each frozen tissue $(n=6-8)$ and stored in $10 \%$ neutral buffered formalin for 48 hours (fixation). After fixation, samples underwent a dehydration protocol consisting of 10-15 minutes incubation in increasing ethanol concentrations (50-to-100\%) followed by two 20 -minute incubations in xylenes. Following xylene incubation, samples were incubated in molten paraffin wax for 20 minutes (infiltration) and embedded into blocks. 5-7 $\mu \mathrm{m}$ sections were cut and mounted on charged slides and sections 
stained with hematoxylin and eosin. Histological evaluation included gross morphological assessment which included the following: glomerular hypercellularity and matrix deposition, interstitial hypercellularity, tubulointerstitial calcification, inflammation, and fibrosis. All slides were analyzed using a Nikon Labophot 2 microscope (Nikon Instruments, New York, NY) at magnification $10 \mathrm{X}$ by a trained investigator blinded to the identity of the groups. Images were captured using a LCL-500-LHD digital camera with a PC Method Capture Imaging software (Ludesco, Parkville, MD).

Renal RNA isolation and inflammatory gene expression

Total RNA was extracted from frozen kidney tissue $(50 \mathrm{mg})$ using the Zymo Research Direct-zol RNA Miniprep Plus Isolation Kit (Irvine, CA, catalog \#R2071) according to the manufacturer's instruction for total RNA isolation. Isolated RNA integrity was visualized on a $1.5 \%$ agarose gel and quantified by spectrophotometry (NanoDrop 100; Thermo Fisher Scientific, Waltham, MA). Following DNase I treatment with TURBO DNA-free kit (Thermo Fisher Scientific), total mRNA was amplified using the Superscript IV First-Strand Synthesis System with oligo dT primers (Thermo Fisher Scientific).

Real-time quantitative polymerase chain reaction (RT-qPCR) consisted of $2.5 \mu \mathrm{l}$ of SYBR Green Master Mix (Thermo Fisher Scientific), $1 \mu \mathrm{L}$ of cDNA (diluted 1:10), $1 \mu \mathrm{L}$ of respective forward and reverse primers $(10 \mu \mathrm{M})$ and $0.5 \mu$ of deionized distilled water for a total reaction volume of $5 \mu \mathrm{l}$. The reactions were performed in a $7500 \mathrm{ABI}$ Real-Time PCR System (Thermo Fisher Scientific). The thermal profile consisted of $50^{\circ} \mathrm{C}$ for $2 \min , 95^{\circ} \mathrm{C}$ for 10 min then 40 cycles of $95^{\circ} \mathrm{C}$ for $15 \mathrm{sec}$ and $60^{\circ} \mathrm{C}$ for $1 \mathrm{~min}$. A melt curve analysis was applied at the end of cycling. Primers that were designed for transcription factors, nuclear factor kappa-light chain enhancer of B cells (NFKB) and NADPH oxidase 4 (NOX4) and for inflammatory cytokines, tumor necrosis factor-alpha (TNF- $\alpha$ ), and interleukin-6 (IL-6) as well as for housekeeping genes, $\beta$-actin and glyceraldehyde 2-phosphate dehydrogenase (GAPDH) using the Primer3 program 
(Howard Hughes Medical Institute) and respective mRNA sequences obtained by NCBI.

Forward and reverse primers for genes of interest are listed below:

\begin{tabular}{|c|c|c|c|}
\hline Gene & NCBI Gene ID & Forward Primer & Reverse Primer \\
\hline NFkB & 81736 & $\begin{array}{l}\text { 5' } \\
\text { TTATGGGCAGGAT } \\
\text { GGACCTA 3' }\end{array}$ & $\begin{array}{l}\text { 5' } \\
\text { 'CCTTTCAGGGCTTT } \\
\text { GGTTTA 3' }\end{array}$ \\
\hline TNFa & 24835 & $\begin{array}{l}\text { 5' } \\
\text { CACAAGGCTGCTG } \\
\text { AAGATGT 3' }\end{array}$ & $\begin{array}{l}\text { 5' } \\
\text { GAGGGAAGGAAGG } \\
\text { AAGGAAG 3' }\end{array}$ \\
\hline IL-6 & 24498 & $\begin{array}{l}\text { 5' TGGCTAAGGACC } \\
\text { AAGACCAT 3' }\end{array}$ & $\begin{array}{l}5^{\prime} \\
\text { TTGCCGAGTAGAC } \\
\text { CTCATAGTG 3' }\end{array}$ \\
\hline NOX4 & 85431 & $\begin{array}{l}5^{\prime} \\
\text { CCTCCATCAAGCC } \\
\text { AAGATTC 3' }\end{array}$ & $\begin{array}{l}5^{\prime} \\
\text { CTCCAGCCACACA } \\
\text { CAGACTAAC } 3^{\prime}\end{array}$ \\
\hline$\beta$-actin & 81822 & $\begin{array}{l}5^{\prime} \\
\text { TTGCTGACAGGAT } \\
\text { GCACAAG 3' }\end{array}$ & $\begin{array}{l}\text { 5' }^{\prime} \\
\text { CAGTGAGGCCAGG } \\
\text { ATAGAGC 3' }\end{array}$ \\
\hline GAPDH & 24383 & $\begin{array}{l}5^{\prime} \\
\text { TCAAGAAGGTGGT } \\
\text { GAAGCAG 3' }\end{array}$ & $\begin{array}{l}5^{\prime} \\
\text { CCTCAGTGTAGCC } \\
\text { CAGGATG 3' }\end{array}$ \\
\hline
\end{tabular}

Serum and urinary measures of renal function and health

Serum measures of kidney function included: blood urea nitrogen (BUN), creatinine, total protein, calcium, phosphorous, alanine aminotransferase (ALT). Additionally, serum glucose and amylase were measured. Values were determined enzymatically using a commercially available Vet- 16 rotor and quantified by a Hemagen Analyst automated spectrophotometer (Hemagen Diagnostics Inc., Columbia, MD).

Serum and urine uric acid was determined by commercially available enzymatic assay (Cayman Chemical). Briefly, serum and urine samples were aliquoted onto a 96-well plate and incubated for 15 minutes. Reaction was initiated by adding $15 \mu \mathrm{l}$ of uricase and horseradish peroxidase enzyme mixture, and read at an excitation of $535 \mathrm{~nm}$ and an emission of $590 \mathrm{~nm}$ using a BioTek Synergy H1 microplate reader (Winooski, VT). Inter-assay coefficient of variation was $32.1 \%$ for both serum and urine. 
Calcium balance and retention

Rats were fasted overnight and euthanized by carbon dioxide inhalation. Blood was collected by aorta puncture. Collected blood was centrifuged at $1,500 \mathrm{~g}$ for $10 \mathrm{~min}$ at $4^{\circ} \mathrm{C}$ to obtain serum. Serum samples were stored at $-80^{\circ} \mathrm{C}$ until analyzed. Serum calcium was determined enzymatically using a commercially available Vet- 16 rotor and quantified by a Hemagen Analyst automated spectrophotometer.

During the initial and final weeks of the feeding study, rats were individually housed in metabolic cages to collect urine and feces for $24 \mathrm{~h}$. Initial and final day urine samples were collected, centrifuged at $1,500 \mathrm{~g}$ for $10 \mathrm{~min}$ at $4^{\circ} \mathrm{C}$, filtered through Whatman no. 1 paper, and then diluted 1:10 in $\mathrm{ddH}_{2} \mathrm{O}$. Initial and final feces were collected and dried for $48 \mathrm{~h}$, then ashed in a muffle furnace (model CP18210, Thermolyne, Dubuque, IA) at $550^{\circ} \mathrm{C}$ for $24 \mathrm{~h}$. Fecal samples were then acidified in $70 \%$ nitric acid, neutralized in $\mathrm{ddH}_{2} \mathrm{O}$, filtered through Whatman no. 1 paper, and further diluted $(1: 50 \mathrm{v} / \mathrm{v})$ in $\mathrm{ddH}_{2} \mathrm{O}$. Ca content of feces and urine was determined by ICP.

Urinary calcium excretion was calculated as urinary calcium concentration/urine volume. Calcium apparent absorption was calculated as [(calcium intake-fecal calcium excretion)/(calcium intake) $\times$ 100. Calcium retention was calculated as [(calcium intake - (fecal calcium excretion + urinary calcium excretion)] [24].

Femur morphometry and mineralization

Following $\mathrm{CO}_{2}$ inhalation, the left and right femur were collected, and then defleshed. After no bilateral differences were determined using a t-test with significance set at $p<0.05$, the left femurs were used for all analyses. Femoral morphometry measurements consisting of depth, width, and length were determined using a Vernier caliper (Bel-Art Products, Pequannock, NJ, USA). Length was measured from the medial condyle to the greater 
trochanter. Femurs were weighed using an analytical balance (Mettler Toledo, Columbus, $\mathrm{OH}$, USA).

Total bone mineral was determined by ashing in a muffle furnace at $600^{\circ} \mathrm{C}$ for 24 hours, then weighed ash. To measure specific minerals, bone ash was dissolved in $2 \mathrm{~mL}$ of $70 \%$ nitric acid. Acidified samples were filtered through Whatman no. 1 paper and diluted $(1: 50 \mathrm{v} / \mathrm{v})$ to volume with $\mathrm{ddH}_{2} \mathrm{O}$ and $\mathrm{Ca}$ determined using ICP.

Femur biomechanical strength

Femoral strength indices were assessed using a TA,XT2i Texture Analyzer (Texture Technologies, Scarsdale, NY, USA) fitted with a three point bending apparatus. Femora were placed on supports and force applied to the midshaft marked at a position halfway between the greater trochanter and the distal medical condyle. Bone was broken by lowering a centrally placed blade $(1 \mathrm{~mm}$ width) at a constant crosshead speed $(0.1 \mathrm{~mm} / \mathrm{s})$. The load cell was $50 \mathrm{~kg}$. The load-deflection data were collected by a PC interfaced with the TA,XT2i. Sample test distance was set at $10 \mathrm{~mm}$ with a signal collection rate of 100 points per second. Peak force, ultimate stiffness, ultimate bending stress and Young's modulus were calculated according to Yuan and Kitts [25].

Statistics

Results are expressed as mean \pm standard error of the mean (SEM). Gene expression was determined as a function of $m R N A$ abundance $(A)$, where $A=1 /$ (gene of interest primer efficiency $x \Delta C T$ (g.o.i.) - (average housekeeping primer efficiency $x \Delta C T$ (h.k.)), where the product of efficiency and average of expression of $\beta$-actin was averaged with the product of efficiency and average of expression of GAPDH to determine the overall expression of the two housekeeping gene $[17,26,27]$. Gene expression data for each treatment group were logtransformed prior to statistical analysis. One-way ANOVA was used to determine differences 
among dietary groups. Post hoc multiple comparison tests were performed using Tukey's test with treatment differences considered significant at $p \leq 0.05$ and a tendency at $p=0.08$. All statistical analyses were performed using JMP 12.2 statistical software package (SAS Institute, Cary, NC).

\subsection{Results and Discussion}

Rats are susceptible to renal disease and diets high in fructose and high in calcium have been shown to be detrimental to renal health. Also, and high-fructose diets can detriment bone health $[7,28,29]$. In the current study, histological analysis of the kidneys showed no evidence of fibrosis, glomerular hypercellularity, glomerular matrix deposition, or amyloidosis. However, rats consuming Western diet and diets containing apple pomace showed renal interstitial hypercellularity (Figure 1), suggesting renal inflammation. To further investigate, gene expression of inflammatory transcription factor, NFKB and proinflammatory cytokines, TNF- $\alpha$ and IL-6 as well as NOX4, a highly expressed enzyme regulating generation of reactive oxygen species, were measured in the kidneys. No significant differences were found in renal expression of any of the genes of interest among diet groups (Figure 2). Serum clinical measures of kidney damage and function were also measured [12,30-32]. Serum creatinine, BUN, ALT, and total protein also showed no significant differences among diet groups, collectively indicating absence of inflammation and oxidative stress (Table 4).

Increased fructose consumption and elevated uric acid may play a role in renal inflammation [33-35]. Elevations in uric acid levels have been shown to change the fundamental architecture of renal histology and has been implicated in acute and chronic renal failure [36]. The current study results showed no significant difference in serum or urine uric acid among diet groups (Table 4). Interstitial hypercellularity was observed in 13-29\% of animals, but there were no significant differences in oxidative stress and inflammatory gene expression or serum and urine measurements of renal dysfunction and injury were observed among diet groups. These 
results indicate renal interstitial hypercellularity was unlikely to be of biological significance. Collectively, the results indicate the fructose content of apple pomace was not a risk for renal injury and development of chronic kidney disease in either 'healthy' or Western diet.

In our study, Western diets were high in calcium with Western/AP diet having the highest calcium content (Table 2). Differences in calcium content in diets can have significant effects on calcium excretion, absorption, and retention [37]. Increased calcium excretion can induce nephrocalcinosis through accumulation of calcium in the kidney [38]. Initial urinary and fecal calcium excretion, calcium retention, and calcium absorption showed no significant differences among diet groups (Table 5). At final week, no differences were observed in rats urinary calcium excretion among all groups, but an increase $(p=0.04)$ in fecal calcium excretion by rats consuming a Western/AP diet compared to AIN diet was observed. This was also likely due to a combination of the high insoluble dietary fiber content in apple pomace possibly binding to calcium and the increased dietary calcium in the Western/AP diet. This higher dietary calcium also explains the lack of change in apparent calcium absorption among all diet groups. No differences were observed in calcium retention among all diet groups. Further, renal histological evaluation showed no evidence of calcium deposition in any of the diet groups, further indicating apple pomace consumption to be safe (Figure 2).

While Western diet (high fat and high sugar) and fructose consumption have also been reported to detriment bone health, whole apples have been shown to favorably alter bone health, through increased bone mineral density, decreased calcium loss, and decreased inflammation due to antioxidants present in apples [39-42]. Apple pomace has been shown to contain more calcium than apples [5]. Increasing dietary calcium has been shown to prevent osteoporosis and to lower the risk of bone fractures $[43,44]$. Further, children with adequate calcium consumption have increased bone mineral density $[45,46]$. The present study showed no significant differences in femoral calcium content among diet groups. Additionally, there were no significant differences in femur size or bone strength measurements among diet groups. 
These measures included peak force, ultimate stiffness, ultimate bending stress, and Young's modulus which measures the stiffness and strength of the bones (Table 5).

Another concern is rats consuming Western/AP diets had significantly increased gonadal fat pad weights than rats consuming AIN diets [data not shown, 17]. Obesity and diabetes have been reported to be causal factors in diet-induced kidney disease progression [6,47]. Despite higher adiposity in rats fed Western/AP diet there were no significant differences in fasting serum glucose or amylase among diet groups (data not shown). Our study provides evidence that high fructose and high calcium content of apple pomace was not sufficient to effect renal or bone health in rats, regardless of diet. Studies on apple pomace have reported numerous health benefits including decreases in body weight, as well improvements in serum lipid, insulin, glucose, antioxidant status, and digestion [48-54]. Yet, few studies have evaluated the safety of apple pomace consumption. Devrajan, et al. [55] fed rats unfermented or fermented apple pomace for 2 weeks showed a nonsignificant increase serum BUN, but found no indication of kidney damage [56].

In conclusion, caloric substitution of a healthy or Western diet with $10 \%$ apple pomace had no impact on renal or bone health in growing female rodents. Based on our results apple pomace is safe for consumption, despite its high fructose content combined with a high calcium content, regardless of diet quality. The study provides evidence for apple pomace, a "waste" byproduct of apple processing has a favorable nutritional profile and is safe and therefore has potential to be repurposed as a sustainable food source for human consumption. 


\subsection{References}

1. Bhushan S, Kalia K, Sharma M, Singh B, Ahuja PS. Processing of apple pomace for bioactive molecules. Crit Rev Biotechnol. 2008;28(4):285-296.

doi:10.1080/07388550802368895

2. Carson K, Collins J, Penfield M. Unrefined, Dried Apple Pomace as a Potential Food Ingredient. J Food Sci. 1994;59(6):1213-1215.

3. Grigoras C, Destandau E, Fougere L, Elfakir C. Evaluation of apple pomace extracts as a source of bioactive compounds. Ind Crops Prod. 2013;49:794-804.

doi:10.1016/J.INDCROP.2013.06.026

4. Jane G. Muir *, Susan J. Shepherd, Ourania Rosella, Rosemary Rose, Jacqueline S. Barrett and, Gibson PR. Fructan and Free Fructose Content of Common Australian Vegetables and Fruit. 2007. doi:10.1021/JF070623X

5. Skinner RC, Gigliotti JC, Ku K-M, Tou JC. A comprehensive analysis of the composition, health benefits, and safety of apple pomace. Nutr Rev. August 2018. doi:10.1093/nutrit/nuy033

6. Johnson RJ, Segal MS, Sautin Y, et al. Potential role of sugar (fructose) in the epidemic of hypertension, obesity and the metabolic syndrome, diabetes, kidney disease, and cardiovascular disease. Am J Clin Nutr. 2007;86(4):899-906.

7. Felice JI, Gangoiti MV, Molinuevo MS, McCarthy AD, Cortizo AM. Effects of a metabolic syndrome induced by a fructose-rich diet on bone metabolism in rats. Metabolism. 2014;63(2):296-305.

8. Skinner RC, Gigliotti JC, Ku K-M, Tou JC. A comprehensive analysis of the composition, health benefits, and safety of apple pomace. Nutr Rev. August 2018. 
9. Cockell KA, L'Abbé MR, Belonje B. The Concentrations and Ratio of Dietary Calcium and Phosphorus Influence Development of Nephrocalcinosis in Female Rats. J Nutr. 2002;132(2):252-256.

10. Rafferty K, Walters G, Heaney RP. Calcium Fortificants: Overview and Strategies for Improving Calcium Nutriture of the U.S. Population. J Food Sci. 2007;72(9):R152-R158. doi:10.1111/j.1750-3841.2007.00521.x

11. Drüeke T, Lameire N, Eknoyan G. Chronic Kidney Disease-Mineral-Bone Disorder: A New Paradigm. Adv Chronic Kidney Dis. 2007;14(1):3-12. doi:10.1053/J.ACKD.2006.10.005

12. Odermatt A. The Western-style diet: a major risk factor for impaired kidney function and chronic kidney disease. Am J Physiol Physiol. 2011;301(5):F919-F931.

13. Cordain L, Eaton SB, Sebastian A, et al. Origins and evolution of the Western diet: health implications for the 21st century. Am J Clin Nutr. 2005;81(2):341-354.

14. Filip R, Radzki RP, Bieńko M. Novel insights into the relationship between nonalcoholic fatty liver disease and osteoporosis. Clin Interv Aging. 2018;13:1879-1891. doi:10.2147/CIA.S170533

15. Hashemi Kani A, Alavian SM, Esmaillzadeh A, Adibi P, Azadbakht L. Dietary Quality Indices and Biochemical Parameters Among Patients With Non Alcoholic Fatty Liver Disease (NAFLD). Hepat Mon. 2013;13(7):e10943. doi:10.5812/hepatmon.10943

16. Rinella ME, Sanyal AJ. Management of NAFLD: a stage-based approach. Nat Rev Gastroenterol Hepatol. 2016;13(4):196-205. doi:10.1038/nrgastro.2016.3

17. Skinner R, Warren D, Lateef S, et al. Apple Pomace Consumption Favorably Alters Hepatic Lipid Metabolism in Young Female Sprague-Dawley Rats Fed a Western Diet. Nutrients. 2018;10(12):1882. doi:10.3390/nu10121882 
18. Groothoff JW, Offringa M, van Eck-Smit BLF, et al. Severe bone disease and low bone mineral density after juvenile renal failure. Kidney Int. 2003;63(1):266-275. doi:10.1046/J.15231755.2003.00727.X

19. Rao GN. Diet and Kidney Diseases in Rats. Toxicol Pathol. 2002;30(6):651-656. doi:10.1080/01926230290166733

20. Reeves PG, Nielsen FH, Fahey GC. AIN-93 Purified Diets for Laboratory Rodents: Final Report of the American Institute of Nutrition Ad Hoc Writing Committee on the Reformulation of the AIN-76A Rodent Diet. J Nutr. 1993;123(11):1939-1951. doi:10.1093/jn/123.11.1939

21. Bortolin RC, Vargas AR, Gasparotto J, et al. A new animal diet based on human Western diet is a robust diet-induced obesity model: comparison to high-fat and cafeteria diets in term of metabolic and gut microbiota disruption. Int J Obes. 2018;42(3):525-534. doi:10.1038/ijo.2017.225

22. Department of Health and Human Services. Dietary Guidelines for Americans 20152020 - U.S. Department of Health and Human Services, U.S. Department of Agriculture. New York NY: Skyhorse Publishing; 2017.

23. National Research Council. Guide for the Care and Use of Laboratory Animals: Eighth Edition - National Research Council, Division on Earth and Life Studies, Institute for Laboratory Animal Research, Committee for the Update of the Guide for the Care and Use of Laboratory Animals - Google Books. 8th ed. Washington D.C.; 2010.

https://books.google.com/books?hl=en\&lr=\&id=Vp5mgXtxYdQC\&oi=fnd\&pg=PP2\&dq=national+ research+council+2010+rats\&ots=FrTgd1JCl5\&sig=eZ_vGWk36QfLScXnMbS_BgT8XU\#v=on epage\&q=national research council 2010 rats\&f=false. Accessed February 27, 2018. 
24. Maditz KH, Benedito VA, Oldaker C, et al. Feeding Soy Protein Isolate and n-3 PUFA Affects Polycystic Liver Disease Progression in a PCK Rat Model of Autosomal Polycystic Kidney Disease. J Pediatr Gastroenterol Nutr. 2015;60(4):467-473.

25. Yuan YV, Kitts DD. Estimation of dietary calcium utilization in rats using a biomechanical functional test. Food Chem. 1992;44(1):1-7. doi:10.1016/0308-8146(92)90249-2

26. Jacometo CB, Schmitt E, Pfeifer LFM, et al. Linoleic and $\alpha$-linolenic fatty acid consumption over three generations exert cumulative regulation of hepatic expression of genes related to lipid metabolism. Genes Nutr. 2014;9(4):405. doi:10.1007/s12263-014-0405-7

27. Maditz KH, Benedito VA, Oldaker C, et al. Feeding Soy Protein Isolate and n-3 PUFA Affects Polycystic Liver Disease Progression in a PCK Rat Model of Autosomal Polycystic Kidney Disease. J Pediatr Gastroenterol Nutr. 2015;60(4):467-473.

doi:10.1097/MPG.0000000000000649

28. Bras G, Ross MH. Kidney disease and nutrition in the rat. Toxicol Appl Pharmacol. 1964;6(3):247-262. doi:10.1016/0041-008X(64)90065-1

29. Odermatt A. The Western-style diet: a major risk factor for impaired kidney function and chronic kidney disease Odermatt A. The Western-style diet: a major risk factor for impaired kidney function and chronic kidney disease The Western-Style Diet and Comparison with Other Diets. Am J Physiol Ren Physiol. 2011;301:919-931.

30. Kirtane AJ, Leder DM, Waikar SS, et al. Serum Blood Urea Nitrogen as an Independent Marker of Subsequent Mortality Among Patients With Acute Coronary Syndromes and Normal to Mildly Reduced Glomerular Filtration Rates. J Am Coll Cardiol. 2005;45(11):1781-1786. doi:10.1016/j.jacc.2005.02.068 
31. Rule AD, Larson TS, Bergstralh EJ, Slezak JM, Jacobsen SJ, Cosio FG. Using Serum Creatinine To Estimate Glomerular Filtration Rate: Accuracy in Good Health and in Chronic Kidney Disease. Ann Intern Med. 2004;141(12):929. doi:10.7326/0003-4819-141-12200412210-00009

32. Marcuccilli M, Chonchol M, Marcuccilli M, Chonchol M. NAFLD and Chronic Kidney Disease. Int J Mol Sci. 2016;17(4):562. doi:10.3390/ijms17040562

33. Nakagawa $\mathrm{T}, \mathrm{Hu} \mathrm{H}$, Zharikov $\mathrm{S}$, et al. A causal role for uric acid in fructose-induced metabolic syndrome. Am J Physiol Physiol. 2006;290(3):F625-F631. doi:10.1152/ajprenal.00140.2005

34. Jia G, Habibi J, Bostick BP, et al. Uric Acid Promotes Left Ventricular Diastolic Dysfunction in Mice Fed a Western Diet. Hypertension. 2015;65(3):531-539. doi:10.1161/HYPERTENSIONAHA.114.04737

35. Johnson RJ, Nakagawa T, Sanchez-Lozada LG, et al. Sugar, Uric Acid, and the Etiology of Diabetes and Obesity. Diabetes. 2013;62(10):3307-3315. doi:10.2337/db12-1814

36. Giordano C, Karasik O, King-Morris K, Asmar A. Uric Acid as a Marker of Kidney Disease: Review of the Current Literature. Dis Markers. 2015;2015:382918. doi:10.1155/2015/382918

37. Torre M, Rodriguez AR, Saura-Calixto F. Effects of dietary fiber and phytic acid on mineral availability. Crit Rev Food Sci Nutr. 1991;30(1):1-22. doi:10.1080/10408399109527539

38. Jehle S, Zanetti A, Muser J, Hulter HN, Krapf R. Partial neutralization of the acidogenic Western diet with potassium citrate increases bone mass in postmenopausal women with osteopenia. J Am Soc Nephrol. 2006;17(11):3213-3222. 
39. Wyshak G, Frisch RE. Carbonated beverages, dietary calcium, the dietary calcium/phosphorus ratio, and bone fractures in girls and boys. J Adolesc Health. 1994;15(3):210-215. doi:10.1016/1054-139X(94)90506-1

40. McGartland C, Robson P, Murray L, et al. Carbonated Soft Drink Consumption and Bone Mineral Density in Adolescence: The Northern Ireland Young Hearts Project. J Bone Miner Res. 2003;18(9):1563-1569. doi:10.1359/jbmr.2003.18.9.1563

41. Puel C, Quintin A, Mathey J, et al. Prevention of Bone Loss by Phloridzin, an Apple Polyphenol, in Ovariectomized Rats under Inflammation Conditions. Calcif Tissue Int. 2005;77(5):311-318. doi:10.1007/s00223-005-0060-5

42. Bell JA, Whiting SJ. Effect of fruit on net acid and urinary calcium excretion in an acute feeding trial of women. Nutrition. 2004;20(5):492-493. doi:10.1016/J.NUT.2004.01.015

43. Nguyen T V., Center JR, Eisman JA. Osteoporosis in Elderly Men and Women: Effects of Dietary Calcium, Physical Activity, and Body Mass Index. J Bone Miner Res. 2010;15(2):322331. doi:10.1359/jbmr.2000.15.2.322

44. Feskanich D, Willett WC, Stampfer MJ, Colditz GA. Milk, dietary calcium, and bone fractures in women: a 12-year prospective study. Am J Public Health. 1997;87(6):992-997. doi:10.2105/AJPH.87.6.992

45. Black RE, Williams SM, Jones IE, Goulding A. Children who avoid drinking cow milk have low dietary calcium intakes and poor bone health. Am J Clin Nutr. 2002;76(3):675-680. doi:10.1093/ajen/76.3.675

46. Chan GM. Dietary Calcium and Bone Mineral Status of Children and Adolescents. Arch Pediatr Adolesc Med. 1991;145(6):631. doi:10.1001/archpedi.1991.02160060049019 
47. Ix JH, Sharma K. Mechanisms linking obesity, chronic kidney disease, and fatty liver disease: the roles of fetuin-A, adiponectin, and AMPK. J Am Soc Nephrol. 2010;21(3):406-412. doi:10.1681/ASN.2009080820

48. Rago D, Gürdeniz G, Ravn-Haren G, Dragsted LO. An explorative study of the effect of apple and apple products on the human plasma metabolome investigated by LC-MS profiling. Metabolomics. 2015;11(1):27-39.

49. Ravn-Haren G, Krath BN, Markowski J, et al. Apple pomace improves gut health in Fisher rats independent of seed content. Food Funct. 2018;9(5):2931-2941. doi:10.1039/C7FO01932G

50. Cho KD, Han CK, Lee BH. Loss of body weight and fat and improved lipid profiles in obese rats Fed apple pomace or apple juice concentrate. J Med Food. 2013;16(9):823-830. doi:10.1089/jmf.2013.2784

51. Juśkiewicz J, Żary-Sikorska E, Zduńczyk Z, Król B, Jarosławska J, Jurgoński A. Effect of dietary supplementation with unprocessed and ethanol-extracted apple pomaces on caecal fermentation, antioxidant and blood biomarkers in rats. Br J Nutr. 2012;107(8):1138-1146. doi:10.1017/S0007114511004144

52. Sehm J, Lindermayer H, Meyer HHD, Pfaffl MW. The influence of apple- and red-wine pomace rich diet on mRNA expression of inflammatory and apoptotic markers in different piglet organs. Anim Sci. 2006;82(6):877.

53. Macagnan FT, Santos LR dos, Roberto BS, de Moura FA, Bizzani M, da Silva LP. Biological properties of apple pomace, orange bagasse and passion fruit peel as alternative sources of dietary fibre. Bioact Carbohydrates Diet Fibre. 2015;6(1):1-6. 
54. Ma P, Yao L, Lin X, et al. A mixture of apple pomace and rosemary extract improves fructose consumption-induced insulin resistance in rats: modulation of sarcolemmal CD36 and glucose transporter-4. Am J Transl Res. 2016;8(9):3791-3801.

55. Devrajan A, Joshi VK, Gupta K, Sheikher C, Lal BB. Evaluation of apple pomace based reconstituted feed in rats after solid state fermentation and ethanol recovery. Brazilian Arch Biol Technol. 2004;47(1):93-106. doi:10.1590/S1516-89132004000100013

56. Jagannadha Rao P, Das M, Das S. Jaggery-A Traditional Indian Sweetener. Vol 6.; 2007. http://www.indiansugar.com/sugarstn.htm. Accessed December 11, 2018. 
Table 1. Composition of locally sourced freeze-dried apple pomace.

\begin{tabular}{lc}
\hline \multicolumn{2}{c}{ Macronutrients (\%) } \\
\hline Protein & 3.56 \\
Fat & 1.3 \\
Carbohydrates & 68.1 \\
\hline Sugars (\%) & 32.5 \\
Fructose & 9.77 \\
Glucose & 13.9 \\
Sucrose & $<0.1$ \\
Maltose & $<0.1$ \\
Lactose & \\
\hline Dietary Fiber (\%) & 22.2 \\
Insoluble Dietary Fiber & 11.0 \\
Soluble Dietary Fiber & $\mathbf{0 . 0 2 9}$ \\
\hline Polyphenols (g/kg) & 15.5 \\
\hline Minerals (mg/g) & 1.47 \\
Total Minerals & 1.97 \\
Calcium & 387 \\
Phosphorous & \\
\hline Calories (kcal/100 g)
\end{tabular}


Table 2. Ingredient composition of rodent diets substituted with apple pomace $(10 \% \mathrm{~g} / \mathrm{kg})$ fed to growing female rats.

\begin{tabular}{|c|c|c|c|c|}
\hline \multicolumn{5}{|c|}{ Diet Groups $^{1}$} \\
\hline & AIN & AIN/AP & Western & Western/AP \\
\hline \multicolumn{5}{|l|}{ Key Ingredients ${ }^{1}$} \\
\hline Apple pomace (g/kg) & 0.0 & 100.0 & 0.0 & 100.0 \\
\hline Sucrose $(\mathrm{g} / \mathrm{kg})$ & 100.0 & 43.9 & 340.0 & 283.9 \\
\hline Fructose $(\mathrm{g} / \mathrm{kg})$ & 50 & 54.5 & 170 & 174.5 \\
\hline Total Minerals (mg/g) & 22.1 & 24.2 & 26.4 & 28.0 \\
\hline Calcium (mg/g) & 10.4 & 10.8 & 12.8 & 14.6 \\
\hline Phosphorus (mg/g) & 7.2 & 7.5 & 7.6 & 7.5 \\
\hline \multicolumn{5}{|c|}{ Macronutrients (\% kcal) } \\
\hline Protein & 18.8 & 18.9 & 14.8 & 14.8 \\
\hline Fat & 17.2 & 17.3 & 44.6 & 44.8 \\
\hline Carbohydrate & 63.9 & 63.7 & 40.6 & 40.4 \\
\hline Calories (kcal/g) & 3.8 & 3.7 & 4.7 & 4.7 \\
\hline
\end{tabular}

${ }^{1}$ Abbreviations: AIN, the American Institute of Nutrition; AP, apple pomace. A complete list of ingredients can be found in Supplemental Table 1. 
Table 3. Weekly caloric and macronutrient intake, weekly body weight gain, and kidney and bone weights of growing female rats consuming different diets substituted with apple pomace $(10 \% \mathrm{~g} / \mathrm{kg})$ for 8 weeks.

\begin{tabular}{lccccc}
\hline \multirow{2}{*}{ Measurements } & \multicolumn{5}{c}{ Treatments $^{{ }^{1}}$} \\
\cline { 2 - 6 } & AIN & AIN/AP & Western & Western/AP & $\boldsymbol{p}$-Value \\
\hline Caloric intake (kcal/week) & $368 \pm 11^{\mathrm{b}}$ & $345 \pm 8^{\mathrm{b}}$ & $422 \pm 9^{\mathrm{a}}$ & $430 \pm 17^{\mathrm{a}}$ & $<0.0001$ \\
Initial bwt $(\mathrm{g})$ & $95 \pm 3$ & $92 \pm 3$ & $95 \pm 3$ & $95 \pm 3$ & 0.80 \\
Final bwt $(\mathrm{g})$ & $216 \pm 4$ & $216 \pm 8$ & $229 \pm 5$ & $234 \pm 5$ & 0.08 \\
Average weekly bwt gain (g) & $16 \pm 3$ & $16 \pm 3$ & $18 \pm 3$ & $18 \pm 3$ & 0.94 \\
Average mineral intake (mg/d) & $304.0 \pm 9.3^{\mathrm{b}}$ & $318.8 \pm 7.3^{\mathrm{b}}$ & $368.9 \pm 7.8^{\mathrm{a}}$ & $374.7 \pm 15.0^{\mathrm{a}}$ & $<0.0001$ \\
Right kidney weight (g) & $0.69 \pm 0.02$ & $0.68 \pm 0.02$ & $0.71 \pm 0.02$ & $0.73 \pm 0.02$ & 0.28 \\
Left kidney weight (g) & $0.69 \pm 0.02$ & $0.67 \pm 0.02$ & $0.74 \pm 0.03$ & $0.74 \pm 0.02$ & 0.07 \\
Relative right kidney weight (mg/g) & $0.32 \pm 0.01$ & $0.31 \pm 0.01$ & $0.32 \pm 0.01$ & $0.31 \pm 0.01$ & 0.86 \\
Relative left kidney weight (mg/g) & $0.31 \pm 0.01$ & $0.31 \pm 0.01$ & $0.31 \pm 0.01$ & $0.32 \pm 0.00$ & 0.70 \\
Left kidney ash (mg/g) & $9.86 \pm 0.56$ & $10.07 \pm 0.54$ & $9.14 \pm 1.09$ & $10.34 \pm 0.67$ & 0.71 \\
\hline
\end{tabular}

${ }^{1}$ Values expressed as mean \pm SEM ( $n=6-8$ rats/group). Different superscript letters a and b within the same row. Indicate significant difference at $p \leq 0.05$ by one-way ANOVA followed by Tukey's test. Abbreviations: AIN, American Institute of Nutrition; $\mathrm{AP}$, apple pomace; Bwt, body weight; $\mathrm{CHO}$, carbohydrate. 
Table 4. Effect of consumption of different diets substituted with apple pomace $(10 \% \mathrm{~g} / \mathrm{kg})$ by growing female rats on serum and urine measurements of liver function enzymes, and uric acid following 8 weeks of feeding.

\begin{tabular}{lccccc}
\hline & \multicolumn{5}{c}{ Treatments $^{\mathbf{1}}$} \\
\hline \multicolumn{1}{c}{ Measurements } & AIN & AIN/AP & Western & Western/AP & $\boldsymbol{p}$-Value \\
\hline Serum Creatinine $(\mathrm{U} / \mathrm{L})$ & $1.46 \pm 0.08$ & $1.45 \pm 0.11$ & $1.38 \pm 0.09$ & $1.43 \pm 0.04$ & 0.90 \\
Serum BUN $(\mathrm{mg} / \mathrm{dl})$ & $17.84 \pm 1.59$ & $19.63 \pm 1.41$ & $20.25 \pm 2.32$ & $16.00 \pm 0.94$ & 0.27 \\
Serum ALT $(\mathrm{U} / \mathrm{L})$ & $107.63 \pm 19.59$ & $118.71 \pm 43.60$ & $94.5 \pm 12.58$ & $133.5 \pm 30.59$ & 0.78 \\
Serum Total Protein $(\mathrm{g} / \mathrm{dl})$ & $3.9 \pm 0.25$ & $4.62 \pm 0.34$ & $4.08 \pm 0.67$ & $4.19 \pm 0.34$ & 0.79 \\
Serum Phosphorous $(\mathrm{mg} / \mathrm{dl})$ & $14.18 \pm 0.54$ & $13.46 \pm 1.72$ & $15.68 \pm 0.53$ & $13.09 \pm 1.02$ & 0.35 \\
Serum Calcium $(\mathrm{mg} / \mathrm{dl})$ & $9.56 \pm 0.80$ & $11.10 \pm 1.09$ & $11.49 \pm 0.54$ & $10.51 \pm 1.00$ & 0.48 \\
Serum Uric Acid $(\mu \mathrm{M})$ & $7.24 \pm 0.31$ & $6.27 \pm 1.61$ & $7.19 \pm 0.86$ & $7.57 \pm 1.25$ & 0.86 \\
Urine Uric Acid $(\mu \mathrm{M})$ & $5.94 \pm 2.26$ & $10.35 \pm 2.11$ & $10.40 \pm 1.12$ & $6.79 \pm 1.41$ & 0.23 \\
\hline
\end{tabular}

${ }^{1}$ Values expressed as mean \pm SEM ( $n=4-8$ animals/group). Different superscript letters $a$ and $b$ within the same figure indicates significant difference at $p \leq 0.05$ by one-way ANOVA followed by Tukey's test. Abbreviations: ALT, alanine aminotransferase; BUN, blood urea nitrogen. 
Table 5. Calcium content of serum, feces, urine, and femurs of rats fed different diets substituted with $10 \%$ (g/kg) apple pomace.

\begin{tabular}{lccccc}
\hline \multirow{2}{*}{ Calcium Balance } & \multicolumn{5}{c}{ Treatments $^{1}$} \\
\cline { 2 - 6 } & AIN & AIN/AP & Western $^{\text {Western/AP }}$ & p-Value \\
\hline Ca intake $(\mathrm{g} / \mathrm{d})$ & $12.8 \pm 0.4$ & $13.3 \pm 0.3$ & $12.8 \pm 0.3$ & $13.1 \pm 0.5$ & 0.88 \\
Intake $(\mathrm{mg} / \mathrm{d})$ & $135.6 \pm 4.2^{\mathrm{c}}$ & $140.1 \pm 3.2^{\mathrm{c}}$ & $162.4 \pm 3.5^{\mathrm{b}}$ & $184.9 \pm 7.4^{\mathrm{a}}$ & $<0.0001$ \\
Initial & & & & & \\
Urine Ca excretion (mg/dl) & $0.16 \pm 0.04$ & $0.19 \pm 0.04$ & $0.17 \pm 0.04$ & $0.18 \pm 0.04$ & 0.96 \\
Fecal Ca excretion (mg/d) & $25.9 \pm 3.6$ & $22.9 \pm 3.5$ & $31.3 \pm 3.7$ & $34.7 \pm 2.7$ & 0.12 \\
Ca retention (mg/d) & $89.3 \pm 9.4$ & $94.9 \pm 5.9$ & $96.4 \pm 5.8$ & $109.8 \pm 6.2$ & 0.32 \\
Ca absorption (\%) & $62.5 \pm 4.6$ & $68.0 \pm 4.7$ & $61.4 \pm 4.2$ & $63.3 \pm 3.0$ & 0.70 \\
Final & & & & & \\
Urine Ca excretion (mg/ml) & $0.15 \pm 0.02$ & $0.16 \pm 0.04$ & $0.16 \pm 0.04$ & $0.10 \pm 0.01$ & 0.25 \\
Fecal Ca excretion (mg/d) & $60.9 \pm 2.9^{\mathrm{b}}$ & $79.4 \pm 11.6^{\mathrm{ab}}$ & $81.2 \pm 3.9^{\mathrm{ab}}$ & $99.3 \pm 7.1^{\mathrm{a}}$ & 0.04 \\
Ca retention (mg/d) & $77.7 \pm 5.3$ & $66.7 \pm 5.3$ & $80.8 \pm 5.0$ & $78.9 \pm 5.3$ & 0.25 \\
Ca absorption (\%) & $54.2 \pm 4.1$ & $41.8 \pm 11.8$ & $49.7 \pm 3.2$ & $46.3 \pm 5.3$ & 0.65 \\
\hline
\end{tabular}

${ }^{1}$ Values expressed as mean \pm SEM ( $n=4-8$ animals/group). Different superscript letters a and $b$ within the same figure indicates significant difference at $p \leq 0.05$ by one-way ANOVA followed by Tukey's test. Abbreviations: $\mathrm{Ca}$, calcium. 
Table 6. Femoral morphometry and strength measurements of rats fed different diets substituted with 10\% (g/kg) apple pomace.

\begin{tabular}{|c|c|c|c|c|c|}
\hline \multirow[b]{2}{*}{ Measurement } & \multicolumn{5}{|c|}{ Treatments $^{1}$} \\
\hline & AIN & AIN/AP & Western & Western/AP & $p$-value \\
\hline Length (mm) & $29.71 \pm 0.53$ & $29.09 \pm 0.78$ & $30.52 \pm 0.56$ & $29.36 \pm 0.78$ & 0.09 \\
\hline Medial lateral width (mm) & $2.98 \pm 0.04$ & $3.12 \pm 0.12$ & $3.06 \pm 0.08$ & $3.15 \pm 0.10$ & 0.13 \\
\hline Depth (mm) & $2.78 \pm 0.07$ & $2.73 \pm 0.12$ & $2.60 \pm 0.09$ & $3.06 \pm 0.17$ & 0.43 \\
\hline \multicolumn{6}{|l|}{ Femur mineralization } \\
\hline Ash (mg/g of bone) & $407.92 \pm 11.42$ & $407.75 \pm 9.26$ & $399.66 \pm 7.40$ & $396.94 \pm 6.46$ & 0.80 \\
\hline Calcium ( $\mathrm{mg} / \mathrm{g}$ of bone) & $37.99 \pm 0.78$ & $39.09 \pm 4.41$ & $40.09 \pm 2.26$ & $38.28 \pm 2.08$ & 0.75 \\
\hline \multicolumn{6}{|l|}{ Femur biomechanical strength } \\
\hline Peak force $(\mathrm{N})$ & $1.74 \pm 0.18$ & $1.99 \pm 0.25$ & $1.55 \pm 0.11$ & $1.23 \pm 0.23$ & 0.07 \\
\hline
\end{tabular}




\section{Figure Legend}

Figure 1. Representative histological staining images of the left kidney of growing female rats

consuming (A) AIN, (B) AIN/AP, (C) Western, or (D) Western/AP following 8 weeks of feeding. Black arrows indicate interstitial hypercellularity.

Figure 2. Relative expression of genes involved in inflammation in kidney tissue of young female rats consuming different diets substituted with $10 \% \mathrm{~g} / \mathrm{kg}$ apple pomace for 8 weeks.

Values expressed as mean \pm SEM ( $n=5-7$ animals/group). Abbreviations: AU, arbitrary units, IL6, interleukin-6; NFkB, nuclear factor kappa-light-chain enhancer of activated B cells; NOX4, NADPH oxidase 4; TNF-a, tumor necrosis factor alpha. 


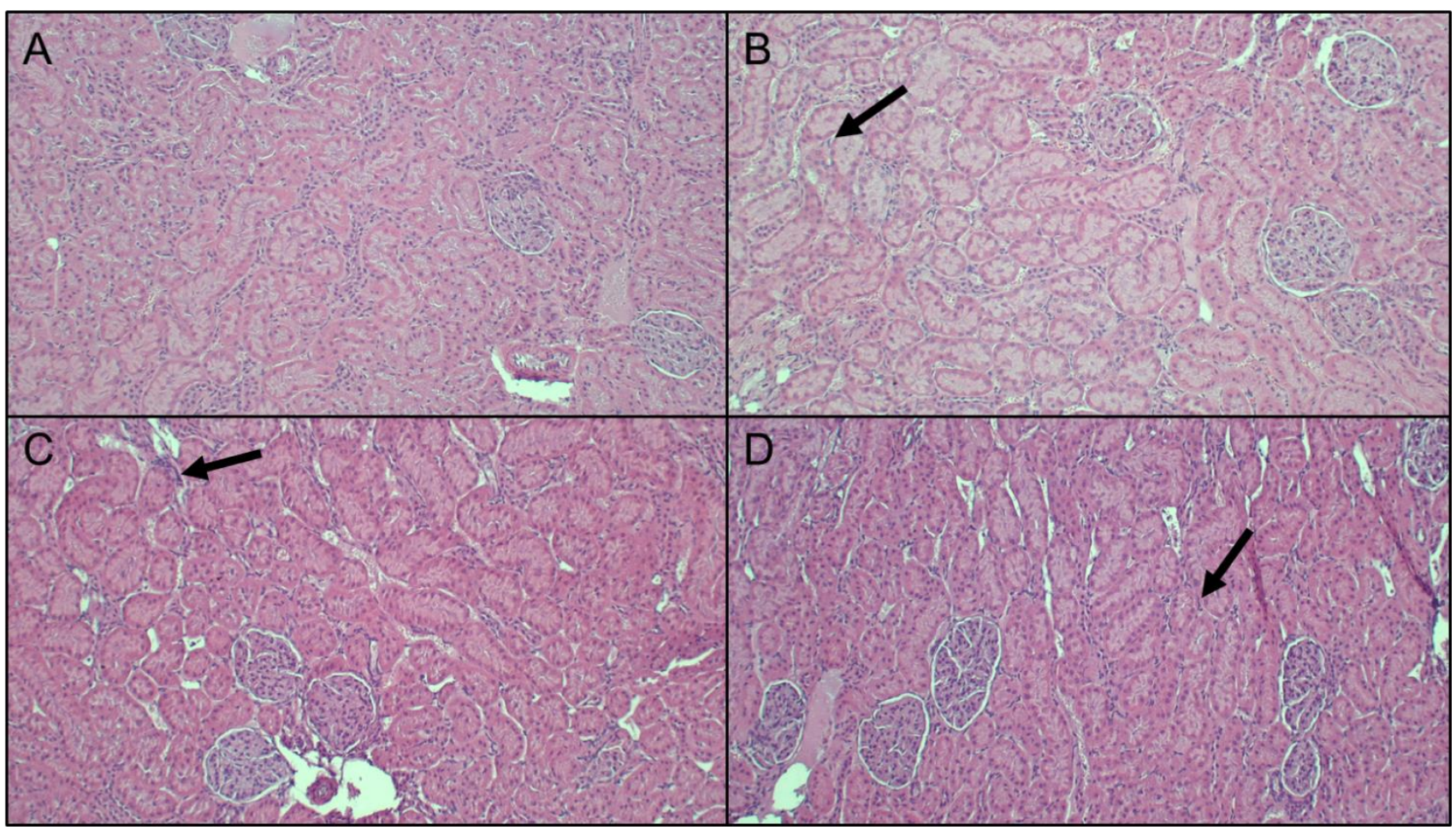

\begin{tabular}{lllll}
\hline Histological changes & AIN & AIN/AP & Western & Western/AP \\
\hline Inflammation & 0 & 0 & 0 & 0 \\
Fibrosis & 0 & 0 & 0 & 0 \\
Glomerular hypercellularity & 0 & 0 & 0 & 0 \\
$\begin{array}{l}\text { Glomerular matrix } \\
\text { deposition }\end{array}$ & 0 & 0 & 0 & 0 \\
Amyloidosis & 0 & 0 & 0 & 0 \\
Interstitial Calcification & 0 & 0 & 0 & 2 \\
Interstitial hypercellularity & 0 & 2 & 1 & \\
\hline
\end{tabular}


aIN 四AIN/AP घWestern घWestern/AP

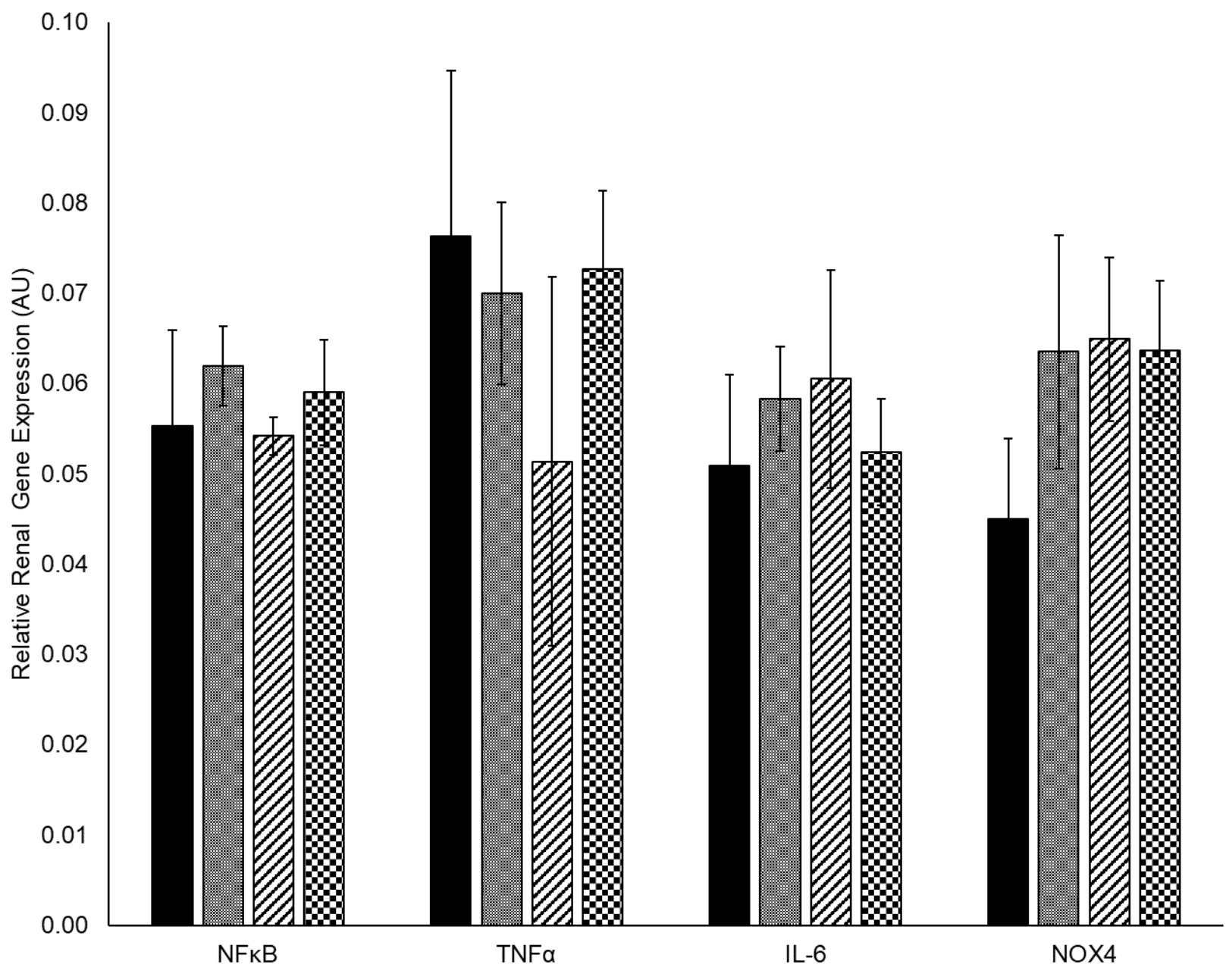




\section{Supplemental Material}

Supplementary Table 1. Composition of rodent diets substituted with apple pomace $(10 \% \mathrm{~g} / \mathrm{kg})$ fed to growing female rats.

\begin{tabular}{lcccc}
\hline & \multicolumn{4}{c}{ Diet Groups } \\
& AIN & AIN/AP & Western & Western/AP \\
\hline Ingredients (g/kg) & & & & \\
Apple pomace & 0.0 & 100.0 & 0.0 & 100.0 \\
Corn Starch & 397.486 & 392.086 & 63.36 & 57.96 \\
Maltodextrin & 132.0 & 132.0 & 60.0 & 60.0 \\
Sucrose & 100.0 & 43.9 & 340.0 & 283.9 \\
Fructose & 50 & 54.45 & 170 & 174.45 \\
Total Dietary Fiber & 50.0 & 50.0 & 50.0 & 50.0 \\
Insoluble Fiber † & 50.0 & 39.0 & 50.0 & 39.0 \\
Soluble Fiber $¥$ & 0.0 & 11.0 & 0.0 & 11.0 \\
Anhydrous Milkfat & 0.0 & 0.0 & 210.0 & 210.0 \\
Soybean Oil & 70.0 & 68.7 & 20.0 & 18.7 \\
Casein & 200.0 & 196.0 & 195.0 & 191.0 \\
L-Cystine & 3.0 & 3.0 & 3.0 & 3.0 \\
Vitamin Mix & 10.0 & 10.0 & 12.5 & 12.5 \\
Mineral Mix & 35.0 & 35.0 & 43.0 & 43.0 \\
Total Minerals & 22.1 & 24.2 & 26.4 & 28.0 \\
Calcium & 10.4 & 10.8 & 12.8 & 14.6 \\
Phosphorous & 7.2 & 7.5 & 7.6 & 7.5 \\
Choline Bitartrate & 2.5 & 2.5 & 3.1 & 3.1 \\
TBHQ, antioxidant & 0.014 & 0.014 & 0.04 & 0.04 \\
Polyphenols & 0.0015 & 0.0029 & 0.0008 & 0.0032 \\
\hline Macronutrients (\% kcal) & & & & \\
Protein & 18.8 & 18.9 & 14.8 & 14.8 \\
Fat & 17.2 & 17.3 & 44.6 & 44.8 \\
Carbohydrate & 63.9 & 63.7 & 40.6 & 40.4 \\
\hline Calories (kcal/g) & 3.8 & 3.7 & 4.7 & 4.7 \\
\hline
\end{tabular}

"Abbreviations: AIN, the American Institute of Nutrition; AP, apple pomace; TBHQ, tert-

butylhydroquinone. $†$ Insoluble fiber is cellulose. $¥$ Soluble fiber is mainly pectin ${ }^{1}$. 


\section{Dissertation Discussion, Conclusions, and Future Directions}

\subsection{Discussion and Conclusions}

This dissertation investigated the health benefits and safety of apple pomace consumption using a rat model. Rats consumed a standard "healthy" diet or a high-fat, highsugar diet typical of most Americans. The Western diet is low in fruits and vegetables, which provide dietary fiber and antioxidants. Finding sustainable food sources with potential to provide increases in these critical nutrients is important for improving diet quality. Study 1 results showed caloric substitution with apple pomace attenuated Western diet-induced NAFLD. Rats consuming Western/AP had decreased histological evidence of steatosis, hepatic triglyceride content, SFA and MUFA content, and hepatic DGAT2 gene expression compared to rats consuming a Western diet. This was attributed to the high fiber content of apple pomace attenuating bile acids.

NAFLD progression to NASH is proposed to be a multiple-hit disease with the onset of the disease due to alteration in hepatic lipid metabolism and subsequent increases in severity due to increased inflammation and oxidative stress. Study 2 results showed apple pomace reduced histological evidence of hepatic inflammation, modulated adipose release of SFA and MUFA and deposition in the liver of rats consuming the Western diet. Caloric substitution with $10 \%$ apple pomace also downregulated gene expression of inflammatory NFKB, IL-6, and TNFa in liver and adipose tissue in rats consuming a Western diet. This was due to increased serum and urine total antioxidants in the Western/AP diet group compared to the Western diet group, attributed to the polyphenol content of apple pomace.

Progression of liver disease can result in systemic damage to the kidneys and bone. Study 3 showed Western diet consumption resulted in NAFLD and progression to NASH but this did not produce detrimental effects in the kidney and bone. Caloric substitution with apple 
pomace, which is high in calcium but also high in fructose, did not produce any significant changes in kidney or bone health, regardless of diet quality. Based on the three studies, we conclude:

1) apple pomace consumption can attenuate diet-induced NAFLD in rats consuming a Western diet,

2) apple pomace consumption can prevent progression of NAFLD to NASH in rats consuming a Western diet,

3) apple pomace consumption is safe for renal or bone health in rats consuming a healthy or Western diet.

Collectively, the study results indicate apple pomace's potential to be a sustainable functional food and nutritional aid for human consumption. The current environmental pollution and costs associated with apple pomace wastage and disposal are unnecessary. Repurposing apple pomace for human consumption provides a solution to a multifaceted problem. Utilizing apple pomace as a functional food can improve health while generating revenue. Methods of utilizing "waste products" is an expanding field as sustainability moves to the forefront of science and society due to a growing population. By providing a better understanding of the physiological effects of consuming products such as apple pomace the public can move towards a more sustainable and healthier world.

\subsection{Potential Future Studies}

Repurposing apple pomace for human consumption is an apparent solution to the increasing waste due to processing of apples for juices, ciders, sauces, and other products. Future studies should address long-term consequences of apple pomace consumption using animal models and human subjects. Human studies should address biological effects of apple 
pomace and investigate consumer acceptability. Marketing and product development studies should be conducted to determine the best method for consumer delivery of apple pomace. Lastly, methods for quick storage must be addressed due to rapid spoilage of apple pomace. Although large scale apple producers may have access to industrial freeze-driers, small apple farmers do not. Addressing ways to improve apple pomace drying affordability will be paramount for enabling apple farmers to effectively repurpose apple pomace, currently a waste byproduct of apple processing, for human consumption. 